



\section{A new male in the group: a blessing or a burden?}

The role of resident female behaviour

in male group entry in primates

Astrid Rox 



\section{A new male in the group: a blessing or a burden?}

The role of resident female behaviour

in male group entry in primates

\section{Een nieuwe man in de groep: een zegen of een vloek?}

De reactie van vrouwelijke apen op een nieuwe man in de groep

(met een samenvatting in het Nederlands)

Proefschrift

ter verkrijging van de graad van doctor aan de Universiteit Utrecht op gezag van de rector maginificus, prof.dr. H.R.B.M. Kummeling, ingevolge het besluit van het college voor promoties

in het openbaar te verdedigen op

woensdag 11 november 2020 des middags te 12.45 uur

door

\section{Astrid Rox}

geboren op 9 augustus 1990 te Apeldoorn 


\section{Promotoren:}

Prof. Dr. Elisabeth HM Sterck

Prof. Dr. Jan AM Langermans 
"Feminism isn't about making women strong.

Women are already strong.

It's about changing the way the world percieves that strength."

G.D. Anderson 
The research described in this thesis was performed at the Animal Science Department at the Biomedical Primate Research Centre, Rijswijk, the Netherlands and the Animal Ecology research group of the Department of Biology, Faculty of Science at the Utrecht University, Utrecht, the Netherlands.

Financial support was provided by EURPRIM-NET2, grant agreement no. 262443. Financial support for publication of this thesis was provided by the Biomedical Primate Research Centre.

Illustrations: F. van Hassel, A. Rox

Lay-out: F. van Hassel

Printed by: Ridderprint BV

ISBN: 978-94-6375-874-1

(C) Astrid Rox, 2020. No parts of this thesis may be reproduced or transmitted, in any form, without permission in writing from the author. 


\section{Table of Contents}

Chapter 1. A general introduction to male group entry in macaques $\ldots \ldots \ldots \ldots$. 9

Chapter 2. Indifferent or involved? The role of resident females in male group

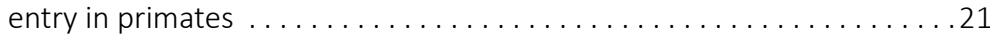

Chapter 3. The male introduction procedure for macaques at the Biomedical Primate Research Centre

Chapter 4. Female social behaviour during three male introductions in captive groups of rhesus macaques

Chapter 5. Factors determining male introduction success and long-term stability in captive rhesus macaques.

Chapter 6. Establishing new between-sex social bonds during male introductions in captive rhesus macaques.

Chapter 7. Female rhesus macaques use mating tactics to regulate female access to a newly introduced male

Chapter 8. A preliminary analysis of species differences in hair cortisol levels during male introductions in female macaques: a link to infanticide . .159

Chapter 9. General discussion and summary .181

References.

Nederlandse samenvatting.

Dankwoord 



\section{A general introduction to male group entry in macaques}




\section{Introduction}

Dispersal, the phenomenon whereby individuals leave their known environment to reside in a new one, is widespread among the animal kingdom. Dispersal promotes gene flow between populations and locations. In this way, inbreeding is prevented, resulting in increased viability and fertility of offspring (Moore and Ali 1984; Pusey 1987; Krause and Ruxton 2002). However, dispersal also involves costs for an individual. Predation risk may be higher in the new environment, and food and safe places are more difficult to find (Isbell 1990; Isbell et al. 1993; Alberts and Altmann 1995; Isbell and van Vuren 1996). When dispersal entails not only a new physical environment, but also entry into a new social group, animals incur additional costs of establishing social relationships with new group members (Isbell and van Vuren 1996, reviewed in: Rox and Sterck in preparation).

The social costs of dispersal may be especially apparent in primates living in multimale multi-female groups, such as capuchins (Sapajus spp. and Cebus spp.), macaques (Macaca spp.), mangabeys (Cerocebus spp.), several baboon species (Papio spp.), and vervets (Cholorocebus spp.). These species live in groups consisting of a number of adult males, several adult females, and their offspring (Mittermeier et al. 2013; Rowe and Myers 2016). Females are philopatric, while males leave their natal group when reaching sexual maturity; a social system found in many primate species (Mittermeier et al. 2013; Rowe and Myers 2016). Males are confronted with unfamiliar resident adult males during group entry, which leads to extensive male-male competition (Dittus 1975; Zhao 1994; van Noordwijk and van Schaik 2000; Georgiev et al. 2016; Marty et al. 2016). This male-male competition is costly to new males, as it may result in severe injuries, a deteriorating body condition, unsuccessful group entry, and sometimes even death (Neville 1968a; Lindburg 1969; Dittus 1975; van Noordwijk and van Schaik 1985, 2000; Zhao 1994; Georgiev et al. 2016; Marty et al. 2016). Additionally, also female behaviour may result in costs for new males. Philopatric females form close social bonds with their female group members (reviewed in: Silk 2002; Massen et al. 2010; Seyfarth and Cheney 2012), but also female-male bonds are common (e.g. Hill 1990; Lemasson et al. 2008; Alberts 2012; Massen and Sterck 2013; Baniel et al. 2016; Haunhorst et al. 2016). Closely bonded and related females generally support each other in conflicts, and may form coalitions in response to social threats or harassment, such as a new male in their group (Bernstein et al. 1977; Packer and Pusey 1979; Sterck et al. 1997). Consequently, resident females may commonly aggress new males in coalitions, but also noncoalitionary aggression from resident females to new males has been observed (Neville 1968a; Bernstein et al. 1977; Packer and Pusey 1979; Wilson and Gordon 1979; Cheney and Seyfarth 1983; Cooper et al. 2001; Fruteau et al. 2010). Moreover, building new social bonds with resident females can be challenging, as females may avoid new males (Bernstein et al. 1977; Packer and Pusey 1979; Wheatley 1982). New males may even be unsuccessful in joining a new group when female group members are too closely bonded (Yamada 1963). Thereby, entering a new social group is a socially challenging situations for males, as interactions with resident males and females may result in large costs and could lead to unsuccessful group entry. 
New and resident male behaviour during group male entry is particularly wellstudied in macaques. Male macaques disperse several times in their lives and are thus regularly confronted with the social costs associated with group entry. They leave their natal group between the age of 3.5 and 6.5 years (Boelkins and Wilson 1972; Drickamer and Vessey 1973; Sprague 1992; Mehlman et al. 1995; Sprague et al. 1998; van Noordwijk and van Schaik 2000). Consequently, males usually experience their first group entry before they grow to full adult body size (i.e. around the age of 9 or 10 (van Noordwijk and van Schaik 1985; Sprague 1992). They may maintain this optimal body condition until they are around 13 years of age, their so-called 'prime' years (Bercovitch 1997). During prime years, males often change groups and attempt to obtain the alpha position in a new group (van Noordwijk and van Schaik 1985; Suzuki et al. 1998). Overall, males typically change groups every 2 to 6 years and disperse several times in their lives before, during and after their prime years (Boelkins and Wilson 1972; Drickamer and Vessey 1973; Dittus 1975; Sprague 1992; Sprague et al. 1998; van Noordwijk and van Schaik 2000). Thereby, males face the challenge to obtain a social position in a new social group every few years. Consequently, the costs and benefits associated with male group entry are important determinants for a male's lifetime fitness.

\section{Male behavioural strategies during group entry}

Although males may migrate regularly, they are not always able to successfully enter a new group (Bernstein et al. 1977; van Noordwijk and van Schaik 1985, 2000). This failure is costly, since they do experience the costs of dispersal, but do not obtain any benefits. To increase their chances of successful group entry, males may use different behavioural strategies. Thereby, they may minimize the social costs and increase the benefits obtained through dispersal. Male macaques may enter a new group as 'unobtrusive' or 'bluff' immigrants (van Noordwijk and van Schaik 1985). Males that enter a group unobtrusively start with a position low in the dominance hierarchy (Drickamer and Vessey 1973; van Noordwijk and van Schaik 1985; Sprague et al. 1998; Suzuki et al. 1998; Hayakawa and Soltis 2011; Marty et al. 2016). They show submission to, and may lose conflicts from, resident males (van Noordwijk and van Schaik 1985). Yet, males entering a group unobtrusively may avoid the costs of extensive male-male competition by submitting to residents, and are never injured during group entry (van Noordwijk and van Schaik 1985; Marty et al. 2016). However, also the fitness benefits they will obtain are low, as unobtrusive immigrants usually remain on the periphery of the group (Drickamer and Vessey 1973). Females may even prevent them from entering the core of the group (Yamada 1971; Packer and Pusey 1979). Males of all age classes may enter unobtrusively (van Noordwijk and van Schaik 1985; Sprague 1992). Yet, this strategy may be particularly used by males that recently left their natal group or males past their prime age, who are unlikely to win male-male competition. Young males that entered a group unobtrusively usually spend several years in a group before they challenge other resident males over dominance. Once they reach prime age and challenge resident males they are often successful in taking 
over their group (van Noordwijk and van Schaik 1985; Marty et al. 2016). Consequently, unobtrusive immigrants may only gain mating access to females, and benefit from group entry, after spending several years in the group (Marty et al. 2016).

When prime aged males disperse and enter a new group, they may enter as bluff immigrants (van Noordwijk and van Schaik 1985; Suzuki et al. 1998). Bluff immigrants attempt to take over the highest dominance rank immediately after arrival (Neville 1968b; van Noordwijk and van Schaik 1985; Sprague 1992; Sprague et al. 1998; Hayakawa and Soltis 2011; Georgiev et al. 2016; Marty et al. 2016). They engage in extensive male-male competition, which may result in severe injuries or deteriorating body condition (van Noordwijk and van Schaik 2000; Hayakawa and Soltis 2011; Georgiev et al. 2016; Marty et al. 2016). Thereby, bluff immigrants experience large costs during group entry. Some bluff immigrants win aggressive encounters with resident males and successfully take over a group (Neville 1968b; Georgiev et al. 2016). However, more often they are unsuccessful or only succeed in taking over smaller groups (van Noordwijk and van Schaik 1985, 2000; Hayakawa and Soltis 2011). Yet, when successful, bluff immigrants gain mating access to the females right after group entry (Neville 1968b; Hayakawa and Soltis 2011; Georgiev et al. 2016) and gain immediate fitness benefits. This strategy may be especially beneficial for strong and prime aged males, who have high chances of winning male-male competition. Although exceptional, males may even delay their dispersal to reach optimal body size and be more successful in taking over a new group (Marty et al. 2017a).

Thus, macaque males may use different behavioural strategies when entering a new group, affecting the costs and benefits they will experience during group entry. Studies reporting on the costs and benefits associated with these different male strategies focus on the costs of male-male competition, and assume that males immediately gain mating benefits when obtaining the alpha position. Yet, this rules out female mate choice, a well-established phenomenon in primates (Clutton-Brock and McAuliffe 2009), and ignores that females may respond to the risk associated with new males, such as infanticide and increased male aggression.

\section{Risks and benefits for females}

Male group entry not only yields costs and benefits for new males, also female fitness may be affected. Male group entry can be risky for females, but may also results in benefits. The female attitude towards new males is likely determined by the balance between these risks and benefits. The major risks of male group entry for females are high levels of male-female aggression, and infanticide. New males may behave aggressively towards resident females, and even injure them (Lindburg 1969; Rose et al. 1972; Bernstein et al. 1977; Wheatley 1982; Winston 1985; Rox et al. 2019). Additionally, new males may be infanticidal and kill dependent offspring (van Noordwijk and van Schaik 2000; Singh et al. 2006; Zaunmair et al. 2015). Consequently, female macaques may respond aggressively towards new males entering their group, as is reported in the few studies reporting resident female-new male interactions (Neville 1968b; Bernstein et al. 1977; Packer and Pusey 1979) and even injure them (Packer 
and Pusey 1979; Cheney and Seyfarth 1983). Some studies even report females successfully preventing new males to enter their group (Bernstein et al. 1977; Packer and Pusey 1979). Thus, female macaques may respond aggressively to new males entering their group, which may affect the male's chances of successful group entry.

Females may also obtain benefits from male group entry. New males may be preferred mating partners for resident females (Sprague 1992; Manson 1995; Inoue and Takenaka 2007; Hayakawa and Soltis 2011). Females may benefit from mating with new males as it decreases chances of inbreeding (Packer 1979) and it increases the genetic diversity of their offspring (Clutton-Brock and McAuliffe 2009). Moreover, mating with a new male that successfully took over the alpha position in de the group (i.e. successful bluff immigrant), ensures females they mate with a strong and high quality male (Packer 1979; Henzi and Lucas 1980). Additionally, promiscuous mating with several different males, including new males, may benefit females in general, as it decreases the chances of infanticide (Hrdy 1979; van Belle et al. 2010) and the offspring may receive more paternal care (Packer 1979). To obtain these benefits, females are expected to stimulate the group entry of preferred males.

Thus, females may be at risk during male group entry, but could also benefit from having new males in their group. Females are expected to adjust their behaviour in order to increase the benefits they obtain from male group entry, while decreasing the risks. Female interference with male group entry has indeed been reported in some studies, describing female-new male aggression or a female mating preference for new males (Sprague 1992; Manson 1995; Inoue and Takenaka 2007; Hayakawa and Soltis 2011). However, whether a female experiences costs and benefit during male group entry may differ between individuals. In particular, a female's reproductive state may determine her response towards new males. Females with young infants are more likely to incur costs, as they are at direct risk of infanticide (Hrdy 1977, 1979; Beehner et al. 2005). Consequently, particularly these females may respond aggressively towards new males. Pregnant females, in contrast, may preferentially associate and mate with new males to confuse paternity, a possible counterstrategy against infanticide (Hrdy 1977, 1979; Ebensperger 1998). Fertile females may show a similar response, and even lead new males to the centre of the group, where they can become part of the social unit (MehIman 1986). However, female reproductive status may not be equally important in determining the female response to male group entry in all species, as infanticide risk may differ between species (reviewed in: Lukas and Huchard 2014). But also other factors, such as sexual dimorphism or female dominance over males, may affect the risks associated with male group entry, and thereby determine the female response to new males (Packer and Pusey 1979, reviewed in: Rox and Sterck in preparation). Thus, individual and species characteristics may determine the female response to new males.

Yet, female behaviour during male group entry is a neglected topic in literature and has never been studied systematically. The few studies reporting female-new male interactions during male group entry are generally relatively old studies, often providing descriptive, and thus non-quantitative, information on female behaviour (e.g. Neville 
1968a; Bernstein et al. 1977; Packer and Pusey 1979). Consequently, the effect of resident female behaviour on the costs and benefits of male group entry is barely understood. Yet, it is crucial to gain a clear view on the role of all resident group members during male group entry to fully understand the social costs of male dispersal in macaques. Therefore, systematic studies on female behaviour during male group entry are called for.

\section{Challenges in studying male group entry}

Conducting systematic studies on female behaviour during male group entry is, however, difficult. In the wild, male dispersal and male group entry are unpredictable events (Singh et al. 2006; Marty et al. 2017a). This is especially true for a-seasonally breeding species, where males may change groups throughout the year (e.g. van Noordwijk and van Schaik 1985, 2000). In seasonally breeding species, male group entry generally occurs right before or early in the breeding season, when most females are receptive (e.g. Lindburg 1969; Boelkins and Wilson 1972; Drickamer and Vessey 1973; Dittus 1975; Hayakawa and Soltis 2011). Still, researchers rarely witness male group entry in the wild (Singh et al. 2006). Only long-term studies or studies collecting data on many different study groups may be able to collect sufficient data to describe the behavioural patterns of individual group members during male group entry (e.g. van Noordwijk and van Schaik 1985). Altogether, it is difficult to plan studies on male group entry in the wild. Therefore, it is not surprising that most researchers reporting male group entry focussed on the behaviours with the most obvious fitness consequences; such as male-male competition and infanticide.

As the chances of conducting systematic studies on male group entry in the wild are slim, valuable knowledge on female behaviour during male group entry may be derived from captive studies. Captive primates should be housed as naturalistic as possible, to ensure animal welfare and promote the expression of natural behaviour. This includes social housing, following natural group dynamics and closely mimicking natural migration patterns. For macaques, animals commonly seen at biomedical research facilities, this concerns female philopatry and male dispersal from the natal group. Within these groups, male introductions are necessary to prevent inbreeding, similar to wild groups. Captive introductions can be planned, behaviour between resident and new individuals can be observed more easily in captivity compared to the wild, and behaviour can be videotaped to ensure all interactions are reliably scored. Complex social interactions, such as reconciliation behaviour, show similar patterns in wild and naturalistic captive groups (Aureli et al. 1989; Aureli 1992). This implies that captive studies are meaningful when studying social behaviour, and the obtained results can be translated to wild groups. Moreover, careful documentation of all injuries, illnesses, deaths, and births will provide the opportunity to study the possible fitness consequences of female behaviour during male group entry. Therefore, studies on male introductions in a naturalistic captive setting are a valuable addition to research on male group entry, and contributes to our understanding on the role resident females play during male group entry. 
Moreover, studying male introductions may contribute to optimizing behavioural management strategies of captive primate groups and increase animal welfare. Housing animals in naturalistic groups promotes the expression of natural species typical behaviour, a commonly used marker of animal welfare (Olsson and Westlund 2007; Fraser 2008). Understanding the animal's natural group composition and sociodynamics is crucial to successfully manage captive primate groups (Olsson and Westlund 2007). Yet, managing captive primate groups is challenging, even when natural group composition and migration patterns are closely mimicked. Introductions of new individuals into existing social groups are risky. New males introduced into groups containing multiple adult females may be rejected and unsuccessful in obtaining a new social position in their new group (Bernstein et al. 1977; Rox et al. 2019). Females may injure new males or, in extreme cases, kill them (records Biomedical Primate Research Centre (BPRC); Rox et al. 2019). But also females and their offspring can be injured during male introductions (BPRC records), and new males may commit infanticide and kill dependent offspring (BPRC records; Zaunmair et al. 2015). Moreover, in the wild, male group entry is associated with high stress levels in new males and resident females (Alberts et al. 1992; Beehner et al. 2005; Marty et al. 2017b). Thus, male introductions may lead to stress and severe, occasionally fatal, injuries. Thereby, these necessary male introductions may compromise animal welfare. To be able to minimize the risks associated with male introductions and optimize introduction success, it is crucial to understand female and male behaviour during introductions. Female-new male interactions may signal females resistance to or liking of new males, and thereby indicate the risks or future success of introductions. Moreover, understanding which factors determine female resistance and which males are particularly liked by females may improve male-to-group matching and increase the chances of successful introduction. Therefore, studying male introductions from an applied point of view will provide important information to optimize captive introduction management and improve animal welfare.

\section{Aim of this thesis}

This thesis aims to gain better understanding of the process of male group entry in primates, particularly in macaques. Therefore, introductions of unfamiliar male rhesus macaques (Macaca mulatta) and long-tailed macaques ( $M$. fascicularis) into naturalistic captive groups are studied at the Biomedical Primate Research Centre (BPRC) in Rijswijk, the Netherlands. The BPRC houses its primates in naturalistic breeding groups wherein natural group composition and migration patterns are closely mimicked. Generally, wild macaques live in multi-male multi-female groups, but also one-male groups are observed in the wild (Neville 1968a; Lindburg 1969; van Noordwijk and van Schaik 1985; Ménard and Vallet 1993; Keane et al. 1997; Singh et al. 2006). During the introductions at the BPRC, one-male groups are created. The male introductions are necessary management procedures to prevent inbreeding, and provide the opportunity to systematically study male group entry under circumstances closely mimicking the wild situation. 
The formation of one-male groups allows us to look beyond male-male competition. This thesis will therefore particularly focus on interactions between resident adult females and new males. Resident female behaviour during male group entry is a neglected topic in literate, yet, female behaviour may affect the costs and benefits males experience during group entry. Additionally, females may prevent successful introductions of new males in captive groups (Bernstein et al. 1977). Therefore, the male introductions will be studied from both an applied (Chapter 3-5) and fundamental (Chapter 2 and 6-8) point of view, with a main focus on the following three questions:

1. How do female macaques respond to male group entry?

2. How can we apply knowledge on female and male behaviour during male group entry to improve captive introduction management?

3. How does female behaviour during male group entry affect new male and female fitness?

The first crucial step in understanding the costs, benefits and consequences of male group entry, is understanding how females respond to new males and why they do so. Therefore, Chapter $\mathbf{2}$ concerns a review on information present in literature regarding female-new male interactions during male group entry in primates. The importance and necessity of systematic studies on female behaviour during male group entry will be stressed in this chapter, while pointing out the current gaps in knowledge on the female role in male group entry. This thesis particularly focusses on filling the gaps in knowledge identified in this review, by conducting behavioural observations on femalenew male interactions during captive male introductions.

Chapter 3 provides a detailed description of the naturalistic housing system at the BPRC, and the BPRC introduction procedure. The housing conditions of the BPRC, where individuals remain in their natal group for life (females) or until puberty (males), are unique among biomedical research institutes world-wide. Understanding of the housing conditions and BPRC introduction procedure is necessary to put the observational studies into perspective and to allow translation of the results to the natural situation. Additionally, other institutes that house captive macaques may use the information provided in this chapter to adjust their own housing system and management procedures. Thereby, male introduction procedures and animal welfare may be improved. The next two chapters elaborate on these male introduction procedures, and aim to apply knowledge on male and female behaviour to improve captive introduction management.

In Chapter 4, the general behavioural pattern observed during three male introductions in rhesus macaques is described. Female-new male behaviour during male introductions may signal when it is safe to leave a male in the group, and may provide information on the long-term stability of the new male's position in the group. This information can be crucial to improve captive introduction management. Chapter 5 presents a retrospective assessment of 64 male introductions that took place in the BPRC breeding colony between 2003 and 2018. Data on the timing of the introductions, 
the characteristics of the group, the characteristics of the new male were, and the outcome the introductions were collected from introduction reports. The study aimed to identify which factors determine the success of the introductions, and long-term stability of the new male's position in the group. The information obtained from this study may help to select males and groups that perform better during introductions, and thereby decrease the risks associated with captive male introductions.

The final part of this thesis approaches the behaviour observed during male introductions from a fundamental point of view. Chapter 6 focusses on the social integration of new males into the existing, closely bonded, social core of the groups. Up to now, many studies have described the existence and benefits of social bods within primate groups (Palombit et al. 1997; Schülke et al. 2010; Mcfarland et al. 2017). Yet, there are no studies describing the establishment of social bonds between adult primates. Female-male bonds are common among macaques (e.g. Massen and Sterck 2013), but not every female builds a good social bond with a male. Understanding how and with whom female-male social bonds are formed, is crucial to understand the fitness consequences of male dispersal. Therefore, social interactions between females and their new male are observed during four male introductions in rhesus macaques, and linked to the bonds established after the introductions.

Another important benefit a new male can obtain after group entry, mating access, is the focus of Chapter 7. Historically, females have been considered as relatively passive participants in mating. Although female mate choice has been well described in primates (see Clutton-Brock and McAuliffe 2009), females are not expected to actively employ mating tactics to obtain access to their preferred male. During the onemale introductions at the BPRC, there is limited access to a highly preferred mating partner; circumstances where female mating tactics can be expected. Therefore, the use of female and male mating tactics is studied, putting the female role in mating into a new perspective. If females indeed use mating tactics during the introductions, they may play an important role in increasing the reproductive benefits of new males.

Next, Chapter 8 elaborates on one of the factors expected to affect female-new male behaviour; infanticide risk. Primate males may kill dependent offspring of resident females (Hrdy 1977, 1979; Fedigan 2003; Ichino 2005; van Belle et al. 2010; Amann et al. 2017; Brasington et al. 2017). Male group entry is therefore stressful for females at risk of infanticide. This stress may help females to respond adequately to the risks associated with new males, but may also have negative fitness consequences on the long-term. However, infanticide risk is not equally high in all species; it is much higher in non-seasonally breeding long-tailed macaques, compared to the seasonally breeding rhesus macaques. We compare the long-term stress response to male group entry between the species, and aim to identify how female-new male interactions mediate the female response to male group entry. Thereby, this last chapter not only focusses on the costs and benefits of male dispersal for the dispersing males, but also investigates the fitness consequences of male group entry for females.

Finally, in Chapter $\mathbf{9}$ the main findings of this thesis are summarized and discussed. By addressing both applied and fundamental questions concerning the female role in 
captive male introductions, a process closely mimicking male group entry in wild macaques, the importance of female-new male behaviour is highlighted. First, tools are provided to improve the welfare and management of captive primate groups at biomedical research facilities. Moreover, the need to explore a more naturalistic housing system for other animal species at research facilities is addressed. Second, the effect of female behaviour on the costs and benefits of male dispersal and the remaining gaps in knowledge are discussed. Overall, this thesis clearly shows that females are active contributors to, and not passive onlookers in, the process of male group entry. 




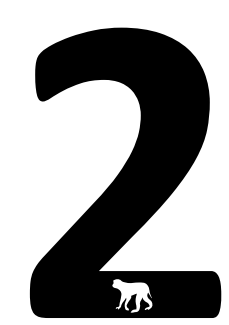

\section{Indifferent or involved? The role of resident females in male group entry in primates}

Astrid Rox, Elisabeth HM Sterck 


\begin{abstract}
Males are the dominant sex in the majority of primate species, while females have often been considered less important or passive group-members. Consequently, female behaviour received less attention from researchers. This sex-bias is especially apparent in studies on male group entry, one of the most challenging situations in a primate's social life. It is evident that resident male behaviour is important during male group entry, yet the role of resident females is understudied. Our review aims to identity the role females play during male group entry. We found that female-new male aggression, mating, and affiliation play a key role in determining the costs, benefits and success of male group entry. Yet it is difficult to generalize the results and draw decisive conclusions, since information is only available on a limited number of species. Overall, systematic studies on social interactions between females and new males are needed. Still, there is emerging evidence that females are active contributors to, and not passive onlookers in, the process of male group entry.
\end{abstract}

\title{
Introduction
}

Males are the dominant sex in the majority of primate species (Mittermeier et al. 2013; Rowe and Myers 2016). Consequently, females have often been considered as less important, sometimes passive, group-members whose only focus is nursing and rearing offspring (Hrdy 1999). It is therefore not surprising that female behaviour received less attention from researchers in the past compared to male behaviour. This sex-bias is especially apparent in studies on male group entry, one of the most challenging situations in a primate's social life.

In many primate species, males generally enter a new group several times in their lives. Dispersing males may experience high costs, such as increased risk of predation (Isbell et al. 1993; Alberts and Altmann 1995). During group entry, they incur additional costs of establishing social relationships with new group members, such as aggression among strangers (Isbell et al. 1993; Isbell and van Vuren 1996). These social costs can be especially high in species living in multi-male multi-female groups; a common social system among primates. Primate males need to obtain a position in a group with a cohesive core of females and multiple males to compete with. In the literature, especially the costs of male-male competition during male group entry are emphasized. Interactions with resident males can result in high costs for new males, in the form of aggression, injuries or even death (Lindburg 1969; Hrdy 1977; Henzi and Lucas 1980; van Noordwijk and van Schaik 1985, 2000; Sprague 1992; Zhao 1994; Gros-louis et al. 2003; Fedigan and Jack 2004; Georgiev et al. 2016).

Incurring these costs of male-male competition does not guarantee a new male any benefits. Males may be unsuccessful in entering a new group and may not be able to access resident females (Jones 1983; van Noordwijk and van Schaik 1985, 2000; Sussman 1992; Fedigan and Jack 2004). Males may be more successful in entering a group they know (Jones 1983). Long-tailed macaque males (Macaca fascicularis) are more successful in re-entering their natal group after a period of semi-solitariness than non-natal males (van Noordwijk and van Schaik 1985, 2000). This may be attributed to 
the presence of male allies. Yet, male allies may also be present in non-natal groups since primate males may disperse together with related peers, or into groups where peers are present (Parga 2010; Albers and Widdig 2013; Gerber et al. 2015). This indicates that not only the presence of male allies, but familiarity with the entire group may promote successful group entry. Primate males are indeed more likely to enter neighbouring groups with whom they are familiar than more distant groups (van Noordwijk and van Schaik 1985, 2000; Richard et al. 1993; Alberts and Altmann 1995). Additionally, hostility towards new males can vary between groups and is likely related to familiarity (Yamada 1971; Cheney and Seyfarth 1982). These studies particularly mention hostility of resident females towards unfamiliar males (Yamada 1971; Cheney and Seyfarth 1982). Female hostility may play an important role in the group entry of new males, as females account for the majority of animals in multi-male multi-female groups and group entry of new males may have fitness consequences for females.

Female interference with male group entry may be linked to female mate choice. Females are philopatric in many primate species living in multi-male multi-female groups (Isbell and van Vuren 1996; Sterck et al. 1997; Mittermeier et al. 2013; Rowe and Myers 2016). These female cannot leave unwanted males to join groups with preferred males, as is observed in species living in one-male groups (Sterck and Korstjens 2000; Qi et al. 2009). Therefore, females may use aggressive or affiliative behaviour to regulate which males enter their group. Females may suffer from extreme aggression, injuries, or even death, when a new male enters a group (Lindburg 1969; Pereira 1983; Alberts et al. 1992; Fedigan and Jack 2004; Fruteau et al. 2010). Moreover, infant mortality may increase drastically after the arrival of a new male due to male infanticide (Hrdy 1977; van Noordwijk and van Schaik 2000; Fedigan 2003; Ichino 2005; Amann et al. 2017; Brasington et al. 2017). Consequently, females may respond with aggression and the formation of coalitions to a new male entering the group, possibly decreasing the chances of successful group entry of unwanted males (Bernstein et al. 1977; Packer and Pusey 1979; Rox et al. 2019). Alternatively, females may facilitate group entry of preferred males by preferentially associating with them. New males may be preferred mating partners for females to decrease the chances of inbreeding (Packer 1979), prevent infanticide (Packer 1979; Hrdy 1979) or to ensure offspring from genetically diverse fathers (Clutton-Brock and McAuliffe 2009). Thus, it can be beneficial for females to hamper of promote new male group entry. However, female-new male behaviour barely received attention in studies on male group entry. When female behaviour is mentioned, it often concerns a few sentences with non-quantitative information in male-focused studies. As a result, female behaviour and its consequences during male group entry are poorly understood. Moreover, the lack of data on female behaviour during male group entry may indicate that female behaviour is irrelevant. A clear view on the role of all group members is crucial to fully understand the costs and benefits males and females experience during male group entry, and understand the fitness consequences of male dispersal (Pereira 1983). This review therefore aims to identity how and under which conditions females intervene with male group entry in primates living in multi-male multi-female groups, by studying female-new male behaviour in literature. 
We expect female-new male behaviour during male group entry to affect the costs, benefits, and success of male group entry. Females are likely to protect themselves and their offspring against new males. The aggressive response of resident females against new males some studies reported (Bernstein et al. 1977; Packer and Pusey 1979; Rox et al. 2018) illustrates that females may not just accept any male into their group. However, whether this female aggression is severe enough to alter a male's costs during group entry is unclear. Females may increase the costs males experience during group entry by injuring them or even prevent successful group entry. Alternatively, females may increase the benefits of male group entry by stimulating social bonding or preferential mating with new males, directly increasing their fitness, and possibly promoting successful group entry. To study the effect of female behaviour on the costs, benefits and success of male group entry Information on female-new male aggression, mating, proximity and affiliation, and female emigration will be extracted from literature. We will focus on male group entry in species naturally living in multi-male multi-female groups. Both wild and captive studies on prosimians, new-world monkeys and old-world monkeys were studied (Table 1).

Whether female behaviour alters the costs, benefits, or success of male group entry may differ between species. Therefore, we will first formulate predictions on species characteristics possibly affecting the female response to new males. Second, we will review the current information on female-new male interactions during male group entry, and link this to the costs and benefits males experience during group entry. Then, we will focus on the long-term consequences of female-new male behaviour and identify whether these interactions affect a male's chances of successful group entry. Finally, we will discuss future directions of research.

\section{Literature search procedure}

We searched for all articles that reported on male group entry in prosimians, newworld monkeys and old-world monkeys living in multi-male multi-female groups with male dispersal. We made use of the Google Scholar, Scopus and Web of Science databases. A wide variety of key-words was used to ensure all possible studies covering female behaviour during male group entry were found, including male group entry, male immigration, male dispersal, male migration, male introduction, male transfer,

Table 1 The 23 species covered in this review, their species characteristics, and whether female behaviour is able to affect the costs, benefits and success of male group entry. Sexual dimorphism is based on information in literature, according $\mathrm{m} / \mathrm{f}$ body weight ratio: \pm 1 is no sexual dimorphism, $<1.3$ is slight sexual dimorphism, 1.3-1.6 is moderate sexual dimorphism, $>1.6$ is strong sexual dimorphism. Infanticide risk is determined based on the presence of literature that (in)directly observed infanticide by new males. When the presence or absence of a certain behaviour is known, this is indicated with y (yes) for present, and $\mathrm{n}$ (no) for absent. When literature describes low levels or rare occurrences of behaviours, both in quantitative values or subjective qualitative terms (e.g. 'low', 'rare', 'uncommon'), this is indicated with I (low). The four most right columns summarize the results, with the number of species wherein the presence of a behaviour is studied, in how many species it was present, in how many species is was low, and in how many species it was absent. y=yes, $\mathrm{n}=\mathrm{no}, \mathrm{l}=\mathrm{low}, \mathrm{no}=\mathrm{none}$, $\mathrm{l}=$ =slight, $\mathrm{m}=$ =moderate, st=strong, $\mathrm{M}=$ male, $\mathrm{nM}=$ new male, $\mathrm{rM}=$ resident male, $\mathrm{F}=$ female 
Table 1

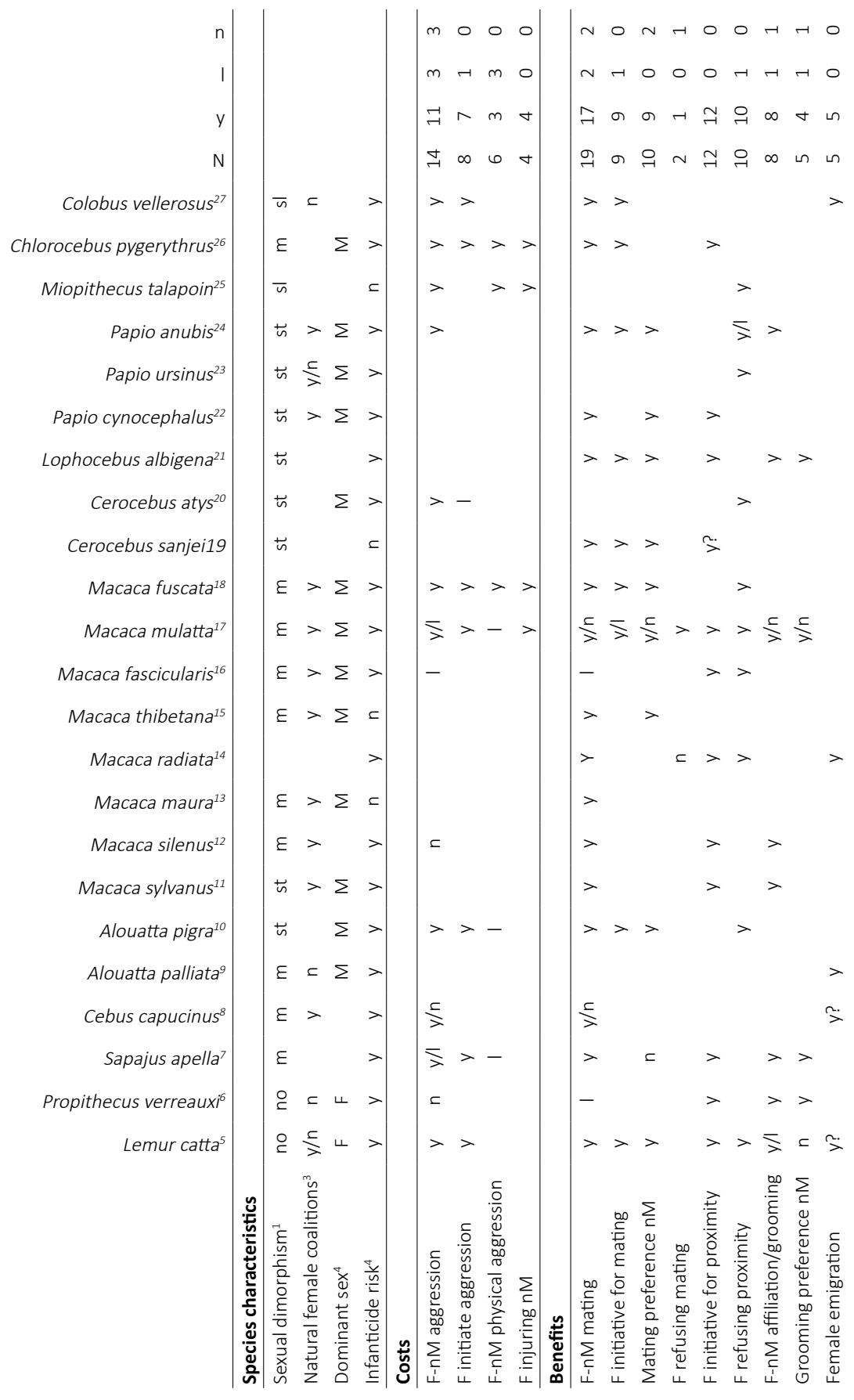


Table 1 continued

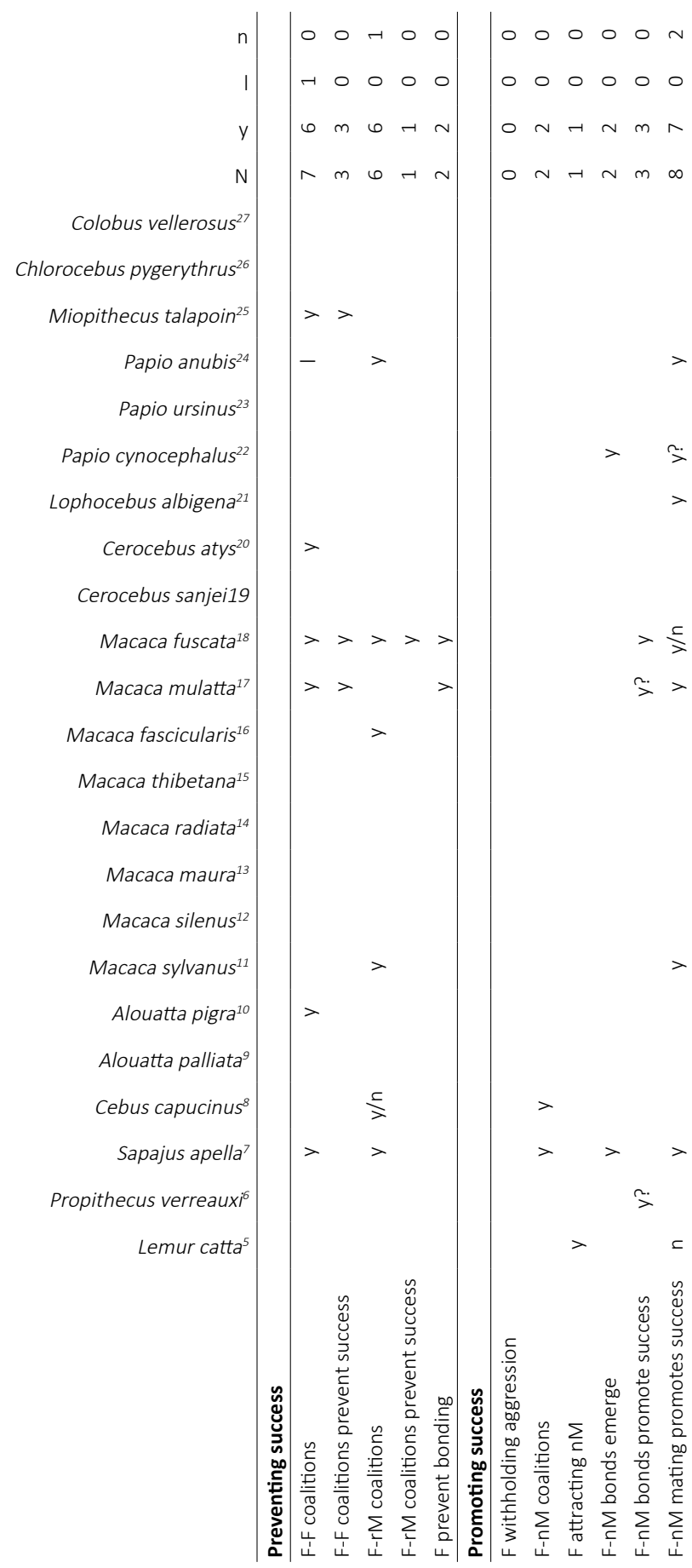




\begin{abstract}
${ }^{1}$ Plavcan and van Schaik 1997; Plavcan 2001; Mittermeier et al. 2013; Rowe and Myers 2016, ${ }^{2}$ Plavcan 2001, ${ }^{3}$ Sterck et al. 1997; Rowe and Myers 2016, ${ }^{4}$ Angst and Thommen 1977; Pereira 1983; Clarke 1983; Camperio Ciani 1984; de Ruiter et al. 1994; Ebensperger 1998; Lewis et al. 2003; Fedigan 2003; Ichino 2005; Singh et al. 2006; Engh et al. 2006; Ramírez-Llorens et al. 2008; Teichroeb and Sicotte 2008; van Belle et al. 2010; Fruteau et al. 2010; Palombit 2012; Arlet et al. 2014; Zaunmair et al. 2015, 5Jones 1983; Pereira and Weiss 1991; Sussman 1992; Gould 1996; Koyama et al. 2002; Ichino 2005; Parga 2010, ${ }^{6}$ Richard et al. 1993; Lewis 2008, ${ }^{7}$ Fragaszy et al. 1994; Cooper et al. 2001; Janson et al. 2012, ${ }^{8}$ (Perry 1998; Fedigan 2003; Gros-louis et al. 2003; Fedigan and Jack 2004; Muniz et al. 2010, ${ }^{9}$ Glander 1992; Clarke and Glander 2010, ${ }^{10}$ van Belle et al. 2010, ${ }^{11}$ Mehlman 1986, ${ }^{12}$ Zaunmair et al. 2015, ${ }^{13}$ Matsumura and Okamoto 1998, ${ }^{14}$ Singh et al. 2006, ${ }^{15}$ Zhao 1994, ${ }^{16}$ Angst 1973; Wheatley 1982; van Noordwijk and van Schaik 1985, 2000, ${ }^{17}$ Neville 1968b, a; Lindburg 1969; Rose et al. 1972; Bernstein and Gordon 1974; Bernstein et al. 1977; Wilson and Gordon 1979; Winston 1985; Rox et al. 2018, 2019, ${ }^{18}$ Yamada 1963, 1971; Sugiyama and Ohsawa 1975; Packer and Pusey 1979; Sprague 1992; Sprague et al. 1998; Suzuki et al. 1998; Hayakawa and Soltis 2011, ${ }^{19} \mathrm{Fernández} \mathrm{2017,}{ }^{20} \mathrm{Fruteau}$ et al. 2010, ${ }^{21}$ Olupot and Waser 2001, 2005, ${ }^{22}$ Pereira 1983; Samuels and Altmann 1986; Alberts et al. 1992; Alberts and Altmann 1995, ${ }^{23}$ Beehner et al. 2005; Engh et al. 2006, ${ }^{24}$ Packer 1979; Packer and Pusey 1979, ${ }^{25}$ Rowell 1974, ${ }^{26}$ Henzi and Lucas 1980; Cheney and Seyfarth 1983, ${ }^{27}$ Saj and Sicotte 2005; Teichroeb et al. 2011; Sicotte et al. 2017
\end{abstract}

male takeover, male replacement, male influx, and infanticide. Additionally, we made use of the reference list of the articles that came up during out database search, because there is only limited information of female behaviour during male group entry present in literature. We searched for any references reporting on male group entry. Any information on interactions between new males and resident females was extracted from the articles resulting from the database search and the search of the reference list.

We found information on female-new male interactions in 23 primate species (Table 1). There is only limited, often non-quantitative information on female behaviour during male group entry available in literature. This makes it difficult to quantify the rates at which females interact with males, and compare those rates between the species. We will compare species based on quantitative data whenever these data are available. Sometimes, researchers classified behaviour rates using subjective, qualitative terms, such as 'high or low levels' or 'many females'. We will use these terms in our review, but use quotation marks and refer to the specific page in the paper mentioning this term. However, we will be mostly forced to describe the presence or absence of female behaviour during male group entry. This will be done by indicating in how many of the studied species the occurrence of the behaviour is studied (i.e. presence or absence mentioned in at least on study), and in how many of those species it was present or absent. The data is summarized in Table 1 . When different studies report different information on the presence or absence of a certain interaction between a female and a new male, both the presence and absence of this behaviour is reported. This may, for example, result in a certain behaviour that has been studied in 10 species, of which the presence is reported in 8 species, and the absence of the same behaviour is reported in 3 species (studied: 10/23 species, present: $8 / 10$ species, absent: $3 / 10$ species). This indicates there may be intra-specific variation in the female response to new males. We indeed expect intra-specific variation; not every female 
may respond the same to a new male, and not every new male may elicit the same response. Yet, information on which or how many females interact with new males is scarce. We will mention this intra-specific variation whenever information is available, but are not able to explain these differences due to lack of in-depth studies. Therefore, this review will mostly report differences in the presence or absence of female-male interactions during male group entry, compare this between species, and link these interactions to the costs, benefits and success of male group entry.

\section{Linking female risks and benefits to species characteristics}

The female response to new males likely depends on the risks associated with male group entry and the benefits females can obtain. The risks of male group entry are mainly related to male aggression towards the females and their offspring. New males may behave aggressively towards resident females (studied: 10/23 species, present: 10/10 species, Sapajus apella: Cooper et al. 2001, Cebus capucinus: Brasington et al., 2017, Macaca fascicularis: Wheatley, 1982, M. mulatta: Rose et al. 1972; Bernstein et al. 1977; Winston 1985; Rox et al. 2018, Cerocebus atys: Fruteau et al. 2010, Papio cynocephalus: Alberts et al. 1992; Pereira 1983, P. ursinus: Beehner et al. 2005, P. anubis: Packer 1979, Colobus vellerosus: Saj and Sicotte 2005, Miopithecus talapoin: Rowell 1974, absent: 0/10 species). New male-female aggression may be 'persistent and intense' (Papio cynocephalus: Pereira 1983 p.93), and new males may be more than 3-4.5 times more aggressive towards females than resident males (Cerocebus atys: Fruteau et al. 2010, Papio cynocephalus: Alberts et al. 1992). Sometimes, this aggression may lead to female injuries (studied: $5 / 23$ species, present: $5 / 5$ species, Sapajus apella: Cooper et al. 2001, Cebus capucinus: Brasington et al. 2017, Macaca radiata: Singh et al. 2006, M. mulatta: Lindburg 1969; Rox et al. 2019, Papio cynocephalus: Alberts et al. 1992; Pereira 1983, absent 1/4 species: Cebus capucinus: Brasington et al. 2017; Fedigan and Jack 2004). Yet, the number of injured females may vary between one, 'some' and 'many' (C. capucinus: Brasington et al. 2017 p.4), and injuries may not be 'severe' (C. capucinus: Brasington et al. 2017 p.4) or not 'serious' (Sapajus apella: Cooper et al. 2001 p.671). In extreme cases, male group entry may even lead to the death or disappearance of 'many' group members (C. capucinus: Brasington et al. 2017 p.4; Fedigan and Jack 2004, Miopithecus talapoin: Rowell 1974). Especially infants may die or disappear during male group entry, as new males may commit infanticide to increase their own reproductive opportunities (Hrdy 1977, 1979; van Noordwijk and van Schaik 2000; Fedigan 2003; Ichino 2005; Singh et al. 2006; Amann et al. 2017; Brasington et al. 2017). However, other studies report that aggression between new males and resident females may be absent ( $C$. capucinus: Brasington et al. 2017), 'rare' (Sapajus apella: Fragaszy et al. 1994 p.411), 'mild' (S. apella: Cooper et al. 2001 p.663), or low (i.e. 0.19 incidences per 30 minutes, Macaca mulatta: Winston 1985). In summary, there is risk of high levels of new malefemale aggression during male group entry, yet, the risks may vary between species or between different occasions of male group entry in the same species. 
Additionally, females may benefit from male group entry. In particular, the new males may be valuable mating partners. First, mating with new males decreased the chances of inbreeding, as new males are likely unrelated (Packer 1979). Second, females may mate with new males to ensure offspring from genetically diverse fathers (Clutton-Brock and McAuliffe 2009). Third, especially strong males will be able to successfully enter their a group (Henzi and Lucas 1980; Rox et al. 2019), and there is more extensive male-male competition in groups with more males. Thereby, the likelihood of mating with a good (i.e. the fittest) male is increased (Packer 1979). Finally, having more males in the group increases the opportunities for promiscuous mating. Promiscuous mating may help females to confuse paternity. Consequently, infanticide risk will be decreased (Hrdy 1979; van Belle et al. 2010) and the offspring may receive more paternal care (Packer 1979). Thus, females may benefit from having a new male in their group, and stimulate male group entry.

These risks and benefits for females associated with male group entry may be affected by species characteristics. Therefore, we expect between species differences in the female response to new males, depending on the sexual dimorphism in the species, the presence of female coalitions, the dominant sex and the risk of infanticide (Figure 1). Based on these species' characteristics, different predictions on the role of females during male group entry can be formulated.

First, the species' sexual dimorphism may influence whether females are able to affect male group entry (Packer and Pusey 1979). Female mate choice can be limited in more sexually dimorphic species (Smuts and Smuts 1993). Therefore, females from sexually dimorphic species may be less inclined to affect male group entry. In addition, these females are also at higher risk when engaging in aggression with new males, and may be less likely to prevent successful group entry. Therefore, females from sexually dimorphic species are not expected to engage in female-new male aggression, and not attempt to prevent new males from entering the group. Instead, they may use affiliation or preferential mating with new males to stimulate bonding and minimize the chances of new male-female aggression. Finally, non-philopatric females from sexually dimorphic species may be more likely to leave their group during male group entry (Figure 1).

Second, the presence of female coalitions against female group members, labelled natural female coalitions, is expected to affect the females' tendency to influence male group entry. In general, primate females are smaller than males (Mittermeier et al. 2013; Rowe and Myers 2016). For smaller single females, the risks of displaying aggression towards new males are high and the chances of winning aggressive encounters are low. The formation of female coalitions, resulting in a communal attack by several females, will decrease the risks associated with female-male aggression and increase the likelihood of winning aggressive encounters. In some species, female coalitions are naturally present in all groups, while female coalitions are uncommon in others (Table 1). Female coalitions against new males may be more easily formed in groups with naturally occurring female coalitions. Moreover, they are used to receiving support from their coalition partners in conflicts with other animals. Consequently, we 


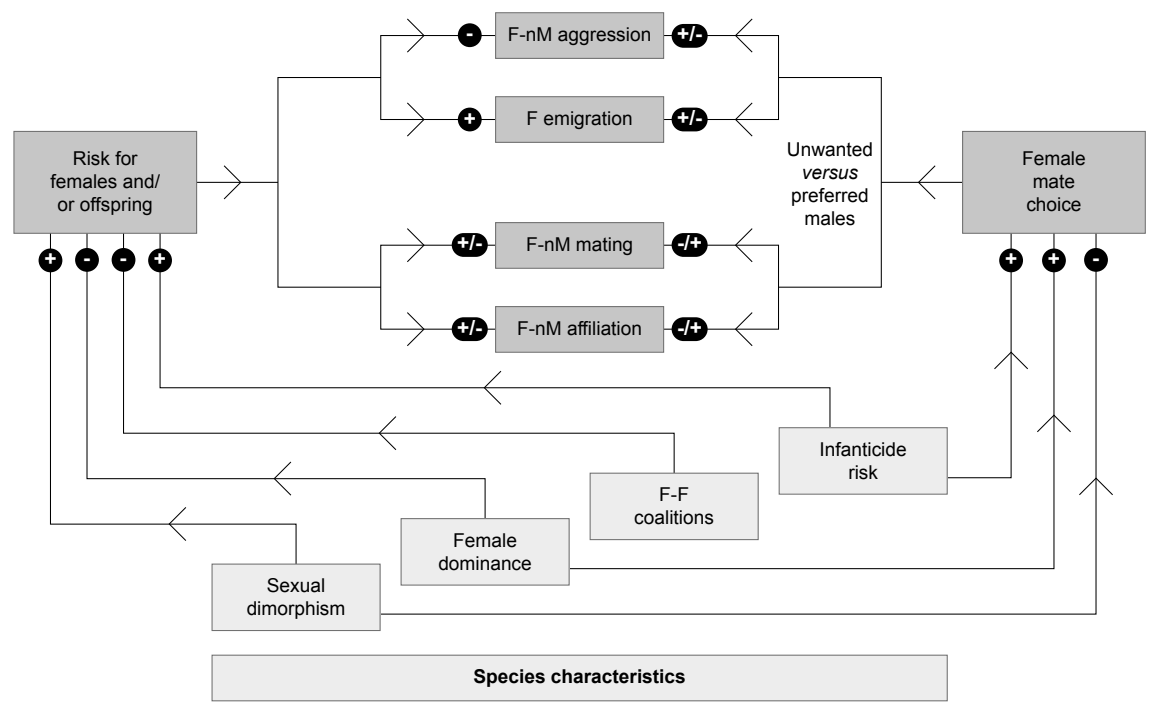

Figure $1 \mathrm{~A}$ schematic overview of the predictions on how species characteristics may affect female-new male behaviour during male group entry. The arrows indicate the direction of the effect. + indicates a positive relationship between two factors, - indicates a negative relationship, +/- indicates that the relationship can either be positive or negative. In case of an effect of female mate choice, this first sign refers to unwanted males / the second sign refers to preferred males.

predict that females from species where female coalitions naturally occur may be more likely to show aggression towards new males. Moreover, the coalitionary back-up may render these females more confident in associating with new males, and engage in mating and affiliation. Female coalitions are not expected to affect female-new male behaviour through mate choice (Figure 1).

Third, female dominance over males may play a role in female influence on male group entry. In species with female dominance, new males pose little risk for females, and females are likely not limited in their mate choice. They are expected to use their dominant position to strictly regulate which males can enter their social group and which males cannot. They may prevent unwanted males from entering their group through aggression, and promote the entry of preferred males through preferentially associating and mating with them. Therefore, females from species with female dominance are expected to have an enhanced influence on the costs, the benefits, and the chances of successful group entry by new males (Figure 1). 
Finally, infanticide risk is expected to affect female behaviour towards new males. New males gain additional reproductive opportunities by committing infanticide (Hrdy 1977), while the loss of an infant is a large cost for females. Infant mortality may increase drastically after a new male entered a group (Engh et al. 2006; Fruteau et al. 2010; van Belle et al. 2010; Amann et al. 2017). Therefore, infanticide is one of the greatest risks for females during male group entry. Females from species with high infanticide risk are expected to display more aggression towards new males and avoid social contact as a counterstrategy against infanticide (Hrdy 1979). Pregnant females may, however, show a different counterstrategy to infanticide and preferentially mate with new males to confuse paternity (Hrdy 1977, 1979). Additionally, females from species with high infanticide risk are expected to be more choosy, and promote the entry of preferred, possible non-infanticidal males, and prevent the entry of unwanted infanticidal males (Figure 1).

These predictions will be tested while extracting information from literature. Yet, there is lack of detailed information in literature. Consequently, we will not be able to systematically address all predictions in relation to all studied behaviours. Therefore, we will only address a prediction whenever there is information available that may support or disprove our predictions.

\section{Female behaviour costly to males}

One of the major costs of male group entry is aggression among strangers (Isbell and van Vuren 1996). Aggression between resident and new males is often severe and well described (Lindburg 1969; Hrdy 1977; Henzi and Lucas 1980; van Noordwijk and van Schaik 1985, 2000; Sprague 1992; Zhao 1994; Gros-louis et al. 2003; Fedigan and Jack 2004), but also resident females may receive aggression from new males and respond aggressively to male group entry. This female-new male aggression may result in costs for new males.

\section{Female-new male aggression}

Female-new male aggression is described in many studies on male group entry, in a variety of primate species (studied: $14 / 23$ species, present: 12/14 species, Lemur catta: Pereira and Weiss 1991; Gould 1996; Ichino 2005, Sapajus apella: Cooper et al. 2001, Cebus capucinus: Brasington et al. 2017, Alouatta pigra: van Belle et al. 2010, Macaca fascicularis: Angst 1973, M. mulatta: Neville 1968a; Bernstein et al. 1977; Wilson and Gordon 1979; Winston 1985; Rox et al. 2018, 2019, M. fuscata: Packer and Pusey 1979, Cerocebus atys: Fruteau et al. 2010, Papio anubis: Packer and Pusey 1979, Miopithecus talapoin: Rowell 1974, Chlorocebus pygerythrus: Cheney and Seyfarth 1983; Isbell et al. 1993, Colobus vellerosus: Saj and Sicotte 2005, absent: 3/14 species, Propithecus verreauxi: Richard et al. 1993; Lewis 2008, Cebus capucinus: Brasington et al. 2017, Macaca silenus: Zaunmair et al. 2015, Table 1). Female vervet monkeys (Chlorocebus pygerythrus) aggress new males 'often' (Cheney and Seyfarth 1983 p.395), up to four times more often than they threaten resident males (Isbell et al. 1993). Additionally, female rhesus macaques (Macaca mulatta) may aggress new males 
more than 30 times in the first hour of contact (Bernstein et al. 1977), yet, elevated aggression levels at the start of male group entry may decrease over time (Bernstein et al. 1977; Rox et al. 2018). Female-new male aggression may be more prevalent than resident male-new male aggression (M. fuscata: Packer and Pusey 1979), although other studies report the opposite (Lemur catta: Jones 1983, Papio anubis: Packer and Pusey 1979). Only few studies report that female-new male aggression is 'rare' (Sapajus apella: Fragaszy et al. 1994 p.411) or happens 'occasionally' during male group entry (Macaca mulatta: Neville 1968a p.2) Only a few females may aggress a new male (1 or 2 out of 8 females in M. fascicularis: Angst 1973), or females may even 'not pay attention' to new males entering a group (Propithescus verreauxi: Richard et al. 1993 p.15). Consequently, female aggression towards new males may be absent ( $P$. verreauxi: Richard et al. 1993; Lewis 2008, Macaca silenus: Zaunmair et al. 2015). In summary, female-new male aggression is often high during new male group entry. Thereby, female-new male aggression has the potential to increase the costs males experience during group entry. Yet, female-new male aggression may differ between species, or between individuals of the same species.

The effect of female-new male aggression on male costs may especially be high when females initiate aggression or injure new males. In all studies that particularly mention the initiator of conflicts, females start fights with new males (studied: 8/23 species, present: 8/8 species, Lemur catta: Pereira and Weiss 1991; Ichino 2005, Sapajus apella: Cooper et al. 2001, Alouatta pigra: van Belle et al. 2010, Macaca mulatta: Neville 1968b, M. fuscata: Yamada 1971; Packer and Pusey 1979, Cerocebus atys: Fruteau et al. 2010, Chlorocebus pygerythrus: Cheney and Seyfarth 1983, Colobus vellerosus: Saj and Sicotte 2005, absent: 0/8 species, Table 1). Females may initiate up to $30 \%$ of the conflicts (C. vellerosus: Saj and Sicotte 2005), while other studied report females even start the majority of the conflicts with new males (Lemur catta: Ichino 2005, Sapajus apella: Cooper et al. 2001). Females may especially initiate aggression to keep new males distant (Aloutta pigra: van Belle et al. 2010, Macaca fuscata: Yamada 1971). Only in sooty managebeys (Cerocebus atys), females are generally only aggressive towards new males after they received aggression, as 98\% of the femalenew male aggression was in response to attacks by new males (Fruteau et al. 2010), yet also these females initiated a few conflicts. Overall, female initiation of aggression towards new males is common, indicating they are able to increase a male's costs during group entry.

In addition, females may injure new males when they physically attack them and thereby jeopardize their health and survival. Female contact aggression towards new males is described in a variety of species (studied: 6/23 species, present: 6/6 species: Sapajus apella: Cooper et al. 2001, Alouatta pigra: van Belle et al. 2010, Macaca mulatta: Bernstein et al. 1977, M. fuscata: Packer and Pusey 1979, Miopithecus talapoin: Rowell 1974, Chlorocebus pygerythrus Cheney and Seyfarth 1983, absent: 0/6 species, Table 1). Yet, contact aggression may only occur 'occasionally' (Sapajus apella: Cooper et al. 2001 p.677). Less than 10\% of all female-new male aggression may be physical, and males may receive physical aggression less than 0.2 times per 
hour (Macaca mulatta: Bernstein et al. 1977). Other studies report that physical female-male aggression only occurs in specific situations such as infanticidal attacks by new males (Alouatta pigra: van Belle et al. 2010), while physical attacks on new males by females seem more common in other studies (Macaca fuscata: Packer and Pusey 1979, Chlorocebus pygerythrus: Cheney and Seyfarth 1983). Especially young males may receive contact aggression (Macaca fuscata: Suzuki et al. 1998), but also prime aged new males can be physically attacked by females ( $M$. fuscata: Packer and Pusey 1979). These attacks can result in 'serious' or 'severe' injuries (M. fuscata: Packer and Pusey 1979 p.214, Chlorocebus pygerythrus: Cheney and Seyfarth 1983 p.395). Some studies even reported new males dying of female inflicted injuries (Macaca mulatta: Rox et al. 2019, Miopithecus talapoin: Rowell 1974). Altogether, these studies imply that female contact aggression is relatively uncommon, but is a considerable risk for new males, as they may be severely or deadly injured.

Female-new male contact aggression was present is all species where it was studied. Yet, the intensity or occurrence differs between the species. Differences in species characteristics may explain why the occurrence of female-new male contact aggression and female-inflicted injuries vary between species. Males may be more likely to be injured in species where sexual dimorphism is low (Packer and Pusey 1979). Indeed, sexual dimorphism is low in the species where females severely injured males (Macaca fuscata and Chlorocebus pygerythrus: Plavcan and van Schaik 1997; Plavcan 2001; Mittermeier et al. 2013; Rowe and Myers 2016). Yet, sexual dimorphism is high in Southern talapoin monkeys, in which females killed new males (Rowell 1974). A study on mandrills (Madrillus sphinx), a species that lives in one-male groups and that shows extreme sexual dimorphism, supports the notion that females can kill a new male even when sexual dimorphism is high (Setchell et al. 2006). Thus, there is no clear connection between sexual dimorphism and females injuring new males. Alternatively, infanticide risk may play a role. Females may be more likely to physically attack new males in defence of their offspring (e.g. Lemur catta: Ichino 2005, Alouatta pigra: van Belle et al. 2010). Yet, male infanticide has not been reported after male group entry in rhesus macaques, while rhesus macaque females are reported to have killed a new male (Rox et al. 2019). Thus, it is apparent that female aggression can affect male costs during group entry as males may be physically attacked, yet, the species characteristics mediating this aggression remain unclear. This is mainly due to lack of studies reporting the initiator of female-new male aggression, and the presence of physical aggression and male injuries.

\section{Female behaviour benefitting males}

Female behaviour may not only result in costs for new males. New males can also benefit from female behaviour, when females promote mating and affiliate with them. Females may use these behaviours to promote the first non-aggressive contact with new males (Henzi and Lucas 1980). 


\section{Female-new male mating}

The entry of a new male in the group can stimulate sexual behaviour in females (Sapajus apella: Fragaszy et al. 1994). Many studies, on a wide variety of species, report females engage in mating with new males (studied: 19/23 species, present: 19/19 species, Lemur catta: Sussman 1992, Propithecus verreauxi: Richard et al. 1993, Sapajus apella: Fragaszy et al. 1994; Cooper et al. 2001, Cebus capucinus: Muniz et al. 2010, Alouatta pigra: van Belle et al. 2010, Macaca sylvanus: Mehlman 1986, M. silenus: Zaunmair et al. 2015, M. maura: Matsumura and Okamoto 1998, M. radiata: Singh et al. 2006, M. thibetana: Zhao 1994, M. fascicularis: van Noordwijk and van Schaik 1985, M. mulatta: Neville 1968b; Rose et al. 1972; Bernstein et al. 1977; Wilson and Gordon 1979; Winston 1985; Rox et al. 2018, M. fuscata: Sugiyama and Ohsawa 1975; Sprague 1992; Sprague et al. 1998; Hayakawa and Soltis 2011, Cerocebus sanjei: Fernández 2017, Lophocebus albigena: Olupot and Waser 2001, Papio cynecephalus: Pereira 1983; Samuels and Altmann 1986, P. anubis: Packer 1979, Chlorocebus pygerythrus: Henzi and Lucas 1980, Colobus vellerosus: Saj and Sicotte 2005; Teichroeb et al. 2011, absent: 2/18 species: Cebus capucinus: Muniz et al. 2010, Macaca mulatta: Neville 1968a; Bernstein et al. 1977; Winston 1985, Table 1). Males often gain mating access soon after group entry, varying from within 1.5 hours to one day (Sapajus apella: Cooper et al. 2001, Macaca mulatta: Bernstein et al. 1977; Georgiev et al. 2016; Rox et al. 2018, Papio anubis: Packer 1979, Colobus vellerosus: Saj and Sicotte 2005), and may mate with all or the majority of the females in the group (Alouatta pigra: van Belle et al. 2010, Macaca radiata: Singh et al. 2006, M. mulatta: Wilson and Gordon 1979, Papio cynocephalus: Samuels and Altmann 1986, Colobus vellerosus: Saj and Sicotte 2005). However, entering a new group does not always guarantee a male mating access. New males may only have 'little access' to females (Macaca fascicularis: van Noordwijk and van Schaik 1985 p.855), mate with part of the females (M. radiata: Singh et al. 2006, Papio cynocephalus: Pereira 1983), or with only one female (Propithecus verreauxi: Richard et al. 1993). Moreover, males may only remain in a group briefly and not sire any offspring (Cebus capucinus: Muniz et al. 2010), not mate at all (Macaca mulatta: Neville 1968a; Bernstein et al. 1977; Winston 1985), or start reproducing after 1 to 3 years (Cebus capucinus: Muniz et al. 2010). Altogether, new males may mate in all species for which the presence or absence of new male mating was studied. Yet, not every female mates with every new male, indicating that not every male obtains mating benefits from entering a new group. Still, males generally benefit from sexual interactions with females, who often engage in high levels of mating with new males.

Female mate choice may explain why not all males benefit from increased mating access after group entry. Female mate choice can be studied by determining with whom females initiate mating, or whether they prefer mating with new males over resident males. Females are reported to take initiative for mating with new males (studied: 9/23 species, present: 9/9 species, Lemur catta: Pereira and Weiss 1991, Alouatta pigra: van Belle et al. 2010, Macaca mulatta: Wilson and Gordon 1979; Winston 1985, M. fuscata: Sprague et al. 1998, Cerocebus sanjei: Fernández 2017, Lophocebus albigena: Olupot and Waser 2001, 2005, Papio anubis: Packer 1979, 
Chlorocebus pygerythrus: Henzi and Lucas 1980, Colobus vellerosus: Saj and Sicotte 2005, absent: 0/9 species, Table 1). Females may even initiate the first sexual interaction with a new male (Chlorocebus pygerythrus: Henzi and Lucas 1980), only initiate sexual interactions with new males (Lemur catta: Pereira and Weiss 1991), or take more initiative than the new males themselves (Cerocebus sanjei: Fernández 2017). Only one study reports that females rarely invite new males to mate, as only one female presented in total three times during 7.5 hours of contact with new males (Macaca mulatta: Winston 1985). Female mating solicitations can increase a new male's mating success (Cerocebus sanjei: Fernández 2017), showing that female-new male interactions may influence new male fitness. This implies that females play an active role in mating with new males and can thereby affect new male fitness.

New males may greatly benefit from sexual interactions with resident females as mating rates with new males are generally high, varying from 4 up to 50 times per hour (Lemur catta: Pereira and Weiss 1991, Macaca mulatta: Bernstein et al. 1977; Rox et al. 2018). This may indicate a female preference for mating with new males. Comparative studies support this, as females generally mate more often with new males than with resident males (Alouatta pigra: van Belle et al. 2010, Macaca thibetana: Zhao 1994, M. mulatta: Wilson and Gordon 1979, Cerocebus sanjei: Fernández 2017, Papio anubis: Packer 1979), and males may have higher mating success in their new group compared to the group they left (Lemur catta: Parga 2010, Macaca fuscata: Hayakawa and Soltis 2011, Lophocebus albigena: Olupot and Waser 2001, Papio cynocephalus: Alberts and Altmann 1995). This female preference for mating with new males may be especially apparent in species with high infanticide risk to confuse paternity, a common counterstrategy to infanticide (Hrdy 1977, 1979). Infanticide after male group entry is indeed observed in many (19/23) of the species showing a mating preference for new males (Table 1). In black howler monkeys (Alouatta pigra) female interest in a new male even increased after he committed infanticide (van Belle et al. 2010), implying a role for infanticide in female mating preference for new males. Taken together, new males are preferential partners and female behaviour can increase new males' reproductive benefits by promoting mating. Infanticide risk may facilitate this behaviour.

Females may, however, also decrease a new male's benefits by refusing mating (studied: 2/23 species, present: 1/1 species, Macaca mulatta: Bernstein et al. 1977, absent: $1 / 1$ species, $M$. radiata: Singh et al. 2006, Table 1). Not all new males may be preferred mating partners. One study did not find increased mating rates during male group entry (Sapajus apella: Cooper et al. 2001), indicating that females did not value the new male more than resident males. Moreover, females may attack unwanted new males attempting to mate (Macaca mulatta: Bernstein et al. 1977). However, one study reports that new males may force females to mate with them (M. radiata: Singh et al. 2006), while using force and aggression to prevent females from rejecting their mating attempt. Taken together, although there is limited information available on females refusing to mate with new males, these three studies illustrate that females may have to the potential to minimize the reproductive benefits males gain during group entry, but males may sometimes overrule female mate choice. 


\section{Female-new male bonding}

Primate males that enter a new social group may be excluded from the social unit encompassing the residents (Bernstein and Gordon 1974). They only become part of the social unit when they form social bonds with resident group members. These social bonds are characterized by frequent affiliation, grooming and proximity, high levels of tolerance, and support in conflicts (Silk 2002; Massen et al. 2012). Females may stimulate bond formation through spending time in proximity with new males and by showing affiliation or, alternatively, prevent the development of social bonds when avoiding contact. Thereby, they may increase or decrease the new male's benefits (Massen et al. 2012; Massen and Sterck 2013; Archie et al. 2014).

Females may approach new males and initiate proximity (studied: 12/23 species, present: 12/12 species, Lemur catta: Pereira and Weiss 1991, Propithecus verreauxi: Lewis 2008, Sapajus apella: Cooper et al. 2001, Macaca sylvanus: Mehlman 1986, M. silenus: Zaunmair et al. 2015, M. radiata: Singh et al. 2006, M. fascicularis: Angst 1973, M. mulatta: Winston 1985, Lophocebus albigena: Olupot and Waser 2001, Papio cynocephalus: Pereira 1983, Chlorocebus pygerythrus: Henzi and Lucas 1980, Colobus vellerosus: Saj and Sicotte 2005, absent: 0/11 species, Table 1). Females may spend 4 to $9 \%$ of their time in proximity of the new male (Sapajus apella: Fragaszy et al. 1994). They may even spend more time with new males than with resident males, and some females take more initiative for social contact than the new males themselves (Propithecus verreauxi: Lewis 2008, Macaca mulatta: Wilson and Gordon 1979, Colobus vellerosus: Saj and Sicotte 2005). This indicates that females prefer proximity with new males over spending time with resident males. This is in line with the previously described mating preference for new males. However, some studies mention that females 'rarely' approach new males (Miopithecus talapoin: Rowell 1974 p.146; 1 or 2 out of 8 females in Macaca fascicularis: Angst 1973), that new males are mainly responsible for maintaining proximity to the females ( $M$. mulatta: Winston 1985, Colobus vellerosus: Saj and Sicotte 2005) or only one female 'frequently' approached the new male (Papio cynocephalus: Pereira 1983 p.95). Another study reports that only females without infants approached new adult males (Macaca radiata: Singh et al. 2006). Unfortunately, there is little information on female proximity to new males available in literature, covering only a few species. Therefore, it is hard to explain the differences between or within species, but infanticide risk may play a role. However, what can be concluded is that females can seek proximity to new males, likely facilitating the start of social bonds.

However, females may also prevent the development of social bonds by refusing proximity to new males (studied: 10/23 species, present:10/10 species, Lemur catta: Gould 1996; Pereira and Weiss 1991, Alouatta pigra: van Belle et al. 2010, Macaca radiata: Singh et al. 2006, M. fascicularis: Wheatley 1982, M. mulatta: Bernstein et al. 1977, M. fuscata: Packer and Pusey 1979; Yamada 1963, 1971, Cerocebus atys: Fruteau et al. 2010, Papio ursinus: Beehner et al. 2005, P. anubis: Packer and Pusey 1979, Miomithecus talapoin: Rowell 1974, absent: 0/9 species, Table 1). Japanese macaque (Macaca fuscata) males often have a hard time establishing new social bonds. New 
males are refused access to the centre of the group, where the females reside (Yamada 1963). Females may aggress new males attempting to enter the centre of the group (Yamada 1971; Packer and Pusey 1979), and can successfully prevent these males to come near in $90 \%$ of the attempts (Packer and Pusey 1979). Similar aggressive responses to new males approaching females have been described in other primate species, and females can succeed in keeping new males distant (Lemur catta: Pereira and Weiss 1991; Gould 1996, Alouatta pigra: van Belle et al. 2010, Papio anubis: Packer and Pusey 1979, Miopithecus talapoin: Rowell 1974). However, in olive baboons (Papio anubis), females may 'usually' be unable to chase new males away (Packer and Pusey 1979 p.214). Instead, when new males are able to enter the centre of the group, females may actively avoid contact with them (Macaca fascicularis: Wheatley 1982, M. mulatta: Bernstein et al. 1977, M. fuscata: Packer and Pusey 1979, Papio anubis: Packer and Pusey 1979). Especially lactating females may avoid new males, which is a possible counterstrategy to infanticide (Macaca radiata: Singh et al. 2006, M. fascicularis: Wheatley 1982, Cerocebus atys: Fruteau et al. 2010, Papio ursinus: Beehner et al. 2005). Yet, infanticide by new males is not equally common in every species where female avoidance of new males has been described (Table 1) and is probably not be the only factor regulating proximity between new males and females. In general, females are able to regulate the proximity with new males by actively seeking or preventing contact. Infanticide risk may contribute to female avoidance of new males.

Whether females actually start bonding during male group entry can be determined by looking at affiliation. All studies focussing on affiliation between new males and resident females, report at least some form of affiliation by females towards new males (studied: 8/23 species, present: 8/8 species, Lemur catta: Pereira and Weiss 1991; Gould 1996, Propithecus verreauxi: Lewis 2008, Sapajus apella: Fragaszy et al. 1994; Cooper et al. 2001; Macaca sylvanus: MehIman 1986, M. silenus: Zaunmair et al. 2015, M. mulatta: Bernstein et al. 1977; Winston 1985; Rox et al. 2018, Lophocebus albigena: Olupot and Waser 2001, Papio anubis: Packer 1979, absent: 0/8 species, Table 1). Moreover, some studies particularly mention the start of social bonds between resident females and new males during male group entry (Macaca silenus: Zaunmair et al. 2015, M. mulatta: Rox et al. 2018). Even though the female's first response to new males is often aggressive, affiliation may replace aggression when the male becomes part of the social unit (M. mulatta: Bernstein et al. 1977; Rox et al. 2018). Females affiliation may even start on the first day the male first enters the group ( $M$. sylvanus: Mehlman 1986, M. mulatta: Bernstein et al. 1977). New males may receive female grooming more than 4 times per hour during the first 10 days he spends with the group (M. mulatta: Bernstein et al. 1977). Affiliation, together with mating, may be the most frequent behaviour during the first two weeks a male spends in his new group ( $M$. mulatta: Rose et al. 1972). 23-46\% of all interactions between resident females and new males may be affiliative (M. mulatta: Bernstein et al. 1977). New males may spend $6 \%$ of their time affiliating (giving and receiving) with resident females (Sapajus apella: Fragaszy et al. 1994) and females may groom new males in $90 \%$ the time they spend in their proximity (Macaca mulatta: Winston 1985). All adult females may affiliate with 
new males daily (Sapajus apella: Fragaszy et al. 1994). Only few studies report that females may be hesitant in affiliating with new males. Affiliation between females and new males only starts after males have been resident for 2.5 months in one study (Lemur catta: Gould 1996), while another study reports that 2 out of 7 females did not groom with their new male (L. catta: Pereira and Weiss 1991). Overall, this illustrates that affiliation between females and new males is common and females play an active role in bonding with new males. Yet, female-new male affiliation may differ between species and not every female may affiliate with new males.

The female role in social bonding with new males will be especially apparent if females show more affiliation than new males, when they preferentially affiliate with new rather than resident males, or when they are able to prevent bonding of new males. Females may groom new males more than new males groom them (Sapajus apella: Cooper et al. 2001), indicating that females take more initiative for bonding. Moreover, new males may be preferred social partners for females, as females may show more affiliation towards new males than towards resident males (Propithecus verreauxi: Lewis 2008, Macaca mulatta: Wilson and Gordon 1979). Female-new male grooming rates may even be 4 times higher than female-resident male grooming rates (Propithecus verreauxi: Lewis 2008). Moreover, males may receive more grooming from females in their new group, than they received in their previous group (Lophocebus albigena: Olupot and Waser 2001). However, new males not always receive frequent affiliation from females. When females are not interested in new males, they may not show any affiliative behaviour (Macaca mulatta: Bernstein et al. 1977). Moreover, females may rarely affiliate with new males, compared to resident males (Lemur catta: Gould 1996). Which factors determine whether new males are preferential social partners or not remains unknown, as there is only limited information on female affiliation towards new males present in literature. However, there is evidence that females often take initiative for affiliation, and often preferentially associate with new males. Thereby, they can facilitate bonding.

\section{Female emigration}

A final way through which females can mediate the benefits of male group entry is through emigration. Females leaving their group after male group entry decrease the number of potential social and mating partners in the group, and thereby decrease the benefits males can obtain. Especially in species where both female and male dispersal occurs, females may leave their group when an unwanted male enters (Teichroeb and Jack 2017). This has previously been described in species living in one-male groups (Sterck and Korstjens 2000; Qi et al. 2009). But also in reported in some species living in multi-male multi-female groups (studied: 2/23 species, present: 2/2 species: Alouatta palliata: Glander 1992, Colobus vellerosus: Sicotte et al. 2017, absent: 0/2 species, Table 1). In multi-male mantled howler groups (Alouatta palliata), females can leave after a new male takes over (Glander 1992). Additionally, female emigration after male group entry is common in white-tighted colobus monkeys (Colobus vellerosus: Sicotte et al. 2017). Even females from species where female philopatry is the norm 
may leave their group after a new male entered. A study on male group entry in white faced capuchins (Cebus capucinus) reports 12 resident group members leaving their home range after a new male entered their group (Fedigan and Jack 2004). The age and sex of the leaving individuals have not been specified. Yet, when considering the average group composition of capuchin groups (e.g. Fedigan 2003; Fedigan and Jack 2004), it is highly likely this leaving group of 12 contained at least some adult females. Female transfer is described in several species were females usually remain in their natal group throughout their life (Lemur catta: Koyama et al. 2002, Propithecus verreauxi: Lewis 2008, Macaca fascicularis: van Noordwijk and van Schaik 1985, Chlorocebus pygerythrus: Isbell et al. 1993). Females may leave their group during socially instable situations (Lemur catta: Koyama et al. 2002), and male group entry may result in social instability and thereby trigger female emigration. Yet, the social context during female emigration remains unreported most studies, making it impossible to study the role of male group entry in a female's decision to leave her group. Additionally, the social instability during male group entry may provide an opportunity for new females to join the group, as was observed in bonnet macaques (Macaca radiata). Possibly, these females were attracted to the group after a new preferred male took over the alpha position from an older, crippled adult male ( $M$. radiata: Singh et al. 2006). Unfortunately, female emigration after male group entry is only systematically studied once, and observed once or twice in other species living in multi-male groups. More studies are needed to draw conclusions on the importance of female dispersal in mediating the benefits of male group entry.

\section{Female behaviour preventing successful male group entry}

Female behaviour may not only affect the costs and benefits males experience during group entry, but also the chances of successful male group entry. There has not been much attention for these consequences of female behaviour during male group entry. Yet, combining the little information present in literature may provide indications of a female influence on a new male's chances of success.

\section{Female coalition formation}

First, females may prevent males from entering a group by using aggression. There are no reports of females individually preventing new males from entering a group in any species. In general, males are larger and stronger than females (Plavcan and van Schaik 1997; Plavcan 2001; Mittermeier et al. 2013; Rowe and Myers 2016). Consequently, individual females are unlikely to win conflicts from males. Yet, females may form coalitions that specifically target new males (studied: $7 / 23$ species, present: 7/7 species, Sapajus apella: Cooper et al. 2001, Alouatta pigra: van Belle et al. 2010, Macaca mulatta: Rox et al. 2018, M. fuscata: Packer and Pusey 1979, Cerocebus atys: Fruteau et al. 2010, Papio anubis: Packer and Pusey 1979, Miopithecus talapoin: Rowell 1974, absent: 0/7 species, Table 1). Coalitions can be involved in up to $27 \%$ of the female conflicts with new males (Macaca fuscata: Packer and Pusey 1979) and can be large, as the majority of females in a group may participate in such coalitions (Sapajus 
apella Cooper et al. 2001). Female coalitions can defeat males up to twice the size of an individual female (Macaca mulatta: Bernstein and Gordon 1974), and prevent them from entering a group (Macaca mulatta: Neville 1968a; Rox et al. 2019, M. fuscata: Packer and Pusey 1979). Yet, female coalitions may mainly be involved in reactive aggression to new males, rather than females proactively forming coalitions to dispel unwanted males. In Japanese macaques, female coalitions are formed in reactions to new males attempting to enter the centre of the group (Yamada 1971). Additionally, a rhesus macaque coalition attacked an unwanted new male when he attempted to mate (Bernstein et al. 1977). Also nonreceptive Southern talapoin (Miopithecus talapoin) females teamed up and defeated new males (Rowell 1974). Moreover, female can form coalitions against new males attempting to commit infanticide (Alouatta pigra: van Belle et al. 2010, Cerocebus atys: Fruteau et al. 2010). However, female coalitions against new males are not equally common in all species. In olive baboons, female coalitions are rare and only occur in $2 \%$ of the female conflicts with new males (Packer and Pusey 1979). Moreover, female coalitions may not always successful in preventing male group entry, yet, reports on unsuccessful female coalitions against new males are lacking. Infanticide risk may affect female coalition formation against new males, as infanticidal attacks may trigger the formation of female coalitions. Yet, female coalitions against new males are also present in species where infanticide after male group entry is not observed (e.g. Macaca mulatta: Rox et al. 2018). Therefore, the factors mediating female coalition formation against new males remain unknown. Still, female coalitions may be an important defence mechanism when a new male enters a group. These coalitions are powerful and can occasionally prevent new males from entering a group.

Second, resident males can join existing female coalitions, and thereby increase the chances of females successfully preventing new male group entry (studied: 2/23 species, present: 2/2 species, Macaca fuscata: Yamada 1971; Packer and Pusey 1979; Suzuki et al. 1998, Papio anubis: Packer and Pusey 1979, absent: 0/2 species, Table 1). Females may receive support from resident males in $8 \%$ (Macaca fuscata) to $12 \%$ (Papio anubis) of their conflicts with new males (Packer and Pusey 1979), while $44-100 \%$ of all resident male-new male aggression may occur with support of female coalitions (Macaca fuscata: Yamada 1971; Packer and Pusey 1979). Rhesus macaque females only aggressed a new male when resident males were in sight (Neville 1968b). This implies that support from resident males can be an important mediator of a female's attitude towards new males. In Japanese macaques, female aggression with the support of resident males is the likely reason why only strong, prime-aged males are successful in entering a new group and obtaining a position high in the dominance hierarchy (Suzuki et al. 1998). Again, this implies an important role for female coalitions, either with resident males or with females, in determining the success of male group entry.

Third, females can also support resident males in conflict with new males (studied: 4/23 species, present: 4/4 species, Sapajus apella: Cooper et al. 2001; Janson et al. 2012, Cebus capucinus: Fedigan and Jack 2004, Macaca sy/vanus: Mehlman 1986, M. fascicularis: van Noordwijk and van Schaik 2000, absent: 1/4 species, Cebus capucinus: 
Gros-louis et al. 2003, Table 1). Females may especially support dominant resident males against new males when new males attempt to take over the alpha position (Macaca fascicularis: van Noordwijk and van Schaik 2000). Female support of resident males is also observed in ongoing conflicts between resident and new males (Cebus capucinus: Fedigan and Jack 2004), but not when resident males attack a new male following the group from a distance (C. capucinus: Gros-louis et al. 2003). This implies that females may especially form coalitions when responding to aggression by new males. Still, the female contribution to male-male conflicts can be valuable for resident males. Female support can prevent or delay the takeover of the alpha position by new males (Sapajus apella: Cooper et al. 2001; Janson et al. 2012, Cebus capucinus: Fedigan and Jack 2004). Especially lactating females may be likely to join resident males in fights with new males (Macaca fascicularis: van Noordwijk and van Schaik 2000). New males can pose a large risk for these females, as they are at risk of infanticide, however, there is too little information present in literature to draw conclusions on the role of infanticide risk on female coalition formation with resident males. Still, it is evident that the female contribution to resident male-new male aggression can be valuable for resident males.

In summary, females may prevent new males from entering groups by forming coalitions with other females or by joining existing coalitions of resident males. Moreover, resident males may support female coalitions. This illustrates, despite the limited information present in literature, that coalitions including resident females can play a key-role during male group entry.

\section{Exclusion from social unit}

Females may prevent successful male group entry by refusing male integration into the social group (i.e. becoming part of the social unit). As described above, females have the power to prevent males from coming close and affiliating with them. Unfortunately, there is little information in literature on the consequences of female refusal to associate with new males. Yet, there is some evidence that the formation of social bonds is important for successful group entry. Close bonds among resident females can prevent males from successfully obtaining a position in the social group (Macaca fuscata: Yamada 1963). Similarly, captive rhesus macaque males can be excluded from the social unit by females during group entry (Bernstein et al. 1977). The females rejected the males and group entry was unsuccessful. Finally, female talapoin monkeys (Miopithecus talapoin) rarely affiliated with new males during unsuccessful introductions (Rowell 1974). Altogether this implies that affiliation between females and the new male may be necessary for successful male group entry. Yet, more research is needed to draw conclusions on whether females that refuse to affiliate with new males have the power to prevent males from entering their group. 


\section{Female behaviour promoting successful group entry}

Females may promote group entry of preferred new males by withholding aggression, forming coalitions with new males, by promoting males to attempt to enter their group, or by stimulating the formation of social bonds through affiliation or preferential mating. There is limited information on this topic present in literature, still, the information present may provide some clues on whether females behaviour can potentially promote male group entry.

\section{Females withholding aggression}

Although aggression levels are generally high at the start of male group entry, some studies report low levels of female-new male aggression (Table 1). Aggression levels may differ within species when comparing males entering different groups (Cebus capucinus: Brasington et al. 2017, Macaca mulatta: Rox et al. 2018), or between different females in the same group (Lemur catta: Ichino 2005). Possibly, females withhold aggression towards specific new males they prefer. Yet, this topic has not received any attention in literature, and needs to be studied in the future.

\section{Female coalitions with new males}

Females may promote successful group entry by forming coalitions with new males against resident males (studied: 2/2 species, present: 2/2 species, Sapajus apella: Cooper et al. 2001, Cebus capucinus: Gros-louis et al. 2003, absent: 0/2 species, Table 1). This has been reported in capuchin monkeys (Sapajus apella: Cooper et al. 2001, Cebus capucinus: Gros-louis et al. 2003) . Additionally, when the alpha male is removed from captive groups, female proximity and coalition formation with subordinate resident males determines which subordinate male becomes the new alpha male (Chlorocebus pygerythrus: Raleigh and McGuire 1989), implying that female coalitions with new males can promote the establishment of a new social position. Moreover, female hostility towards the resident alpha male can destabilize his position and promote take-over by another male (Cebus capucinus: Perry 1998). However, whether females destabilizing the alpha male's position also contributes to successful male group entry remains unknown. Therefore, more studies are needed to draw conclusions on whether primate females use coalition formation to facilitate new male group entry.

\section{Females attracting new males}

Females may promote successful male group entry when they attract new males to their group. Females inviting new males may show less resistance and thereby increase a male's chances to successfully enter the group. Female ring-tailed lemurs reply to calls of dispersing males, and inform them about the location of the group, and thereby may increase the likelihood of male group entry (Jones 1983). Moreover, rhesus macaque females may associate with extra-group males to promote male transfer between groups (Wilson and Gordon 1979). Thus, females may attract new males to their group, but whether this indeed promotes successful group entry needs to be studied in the future. 


\section{Females stimulating bond formation}

Females may facilitate successful group entry by preferentially associating with new males. Affiliating and mating with new males may form the basis for the formation of social bonds, which are expected to promote successful male group entry. Social bonds between resident females and new males can emerge within two weeks after group entry (Sapajus apella: Fragaszy et al. 1994; Cooper et al. 2001, Papio cynocephalus: Samuels and Altmann 1986). These bonds can be strong (Sapajus apella: Fragaszy et al. 1994) and persist for at least 6 months to one year (Macaca mulatta: Rox et al. in prep., Papio cynocephalus: Samuels and Altmann 1986). Female bonding with new males always leads to successful group entry in Japanese macaques (Yamada 1971). Moreover, female Verreaux's sifakas (Propithecus verreauxi) promote group membership of lowranking males by actively seeking contact with them, and grooming them more than dominant resident males (Lewis 2008). Once a first female engages with a new male, more females may follow soon, as one female's interactions with a new male can affect the other females' attitude (Macaca mulatta: Wilson and Gordon 1979). Males may already successfully enter a group when part of the females engage in social interactions (M. mulatta: Rox et al. 2018). This implicates that bonding with one female during group entry may suffice to promote successful group entry.

\section{Mating promoting successful group entry}

Next to affiliation, also mating may promote successful male group entry. Indeed, several researchers particularly mention that mating can help males to enter a new group (studied: 8/23 species, present: 7/8 species, Sapajus apella: Cooper et al. 2001, Macaca sylvanus: Mehlman 1986, M. mulatta: Wilson and Gordon 1979, M. fuscata: Hayakawa and Soltis 2011, Lophocebus albigena: Olupot and Waser 2001, Papio cynocephalus: Samuels and Altmann 1986, P. anubis: Packer 1979, absent: 2/8 species: Lemur catta: Sussman 1992, Macaca fuscata: Sprague 1992, Table 1). This process may be closely linked to affiliation, as especially females that mate with new males engage in grooming (Sapajus apella: Cooper et al. 2001, Macaca fuscata: Sprague 1992; Hayakawa and Soltis 2011, Papio cynocephalus: Samuels and Altmann 1986). Indeed, social bonds between nonreceptive females and new males generally emerge later (Sapajus apella: Cooper et al. 2001). Moreover, females that are fertile or mate with new males can lead them from the periphery to the centre of the group, where they can become part of the social unit (Macaca sylvanus: Mehlman 1986, Papio anubis: Packer 1979). One study even reports that males that do not mate only visit groups for a short period of time, while males that do mate also successfully enter the social group (Lophocebus albigena: Olupot and Waser 2005). However, mating may not always predict whether new males enter a group. Some studies report females mating with visiting males, with males that fail to enter their group, and with males that successfully enter the group (Lemur catta: Sussman 1992, Macaca fuscata: Sprague 1992). Still, the authors of one of these studies mention that mating may help males entering a new group (Sprague 1992). Thus, female enhancement of mating likely facilitates successful male group entry and increases a male's chances of success. 


\section{Discussion}

This review aimed to identity whether females are passive group-members or play an active role during male group entry in primates living in multi-male multi-female groups. To asses this, we studied the effect of female interactions with new males on the costs, benefits and success of male group entry. Female-new male behaviour has barely received attention in studies on male group entry. Papers focusing on male group entry generally elaborate on male-male interactions, while only a few sentences contain information on female behaviour towards new males. As a result, female behaviour and its consequences during male group entry are poorly understood. In this review, we presented evidence that females are active contributors to, and not passive onlookers in, the process of male group entry (Figure 2). Therefore, female behaviour to new males is important and should receive systematic attention.

Females may alter the costs new males experience during group entry, or could hinder male group entry through aggression. Female-new male aggression is, together with mating, the most described female behaviour during male group entry. Generally, females respond highly aggressive to new males and commonly initiate aggression. This results in additional costs for males when aggressively defending themselves. Moreover, females may physically attack males. Physical aggression levels are generally low, but can be a large risk for new males, as females may severely or deadly injure them. Additionally, aggressive females can form coalitions with other females and resident males against new males. These coalitions are often formed in response to new male harassment and can be successful in preventing new males from entering the group. Taken together, it is apparent that female aggression can increase the costs males experience during group entry, and decrease their chances of success.

Additionally, there are indications that females may decrease the costs new males experience by adjusting their aggression levels. This may express female choice. When a preferred male enters the group, females may withhold aggression and lower the new male's costs. Moreover, females may form coalitions with preferred new males to increase their chances of successful group entry. Yet, when females use or withhold aggression to benefit males remains not understood. However, it is clear that not all females always respond aggressively towards all new males. Which individual and species characteristics determine whether a female responds aggressively or not needs to be studied in the future.

Interactions with females can also benefit new males, and increase their chances of successful group entry. First, new males are generally preferred mating partners for females, mating rates with new males are often high and females commonly invite new males to mate. Females behaviour can thus increase a new male's mating access and provide him reproductive benefits. Additionally, several studies indicate that mating increases a male's chances of successful group entry. In contrast, some studies imply that females can also refuse to mate with unwanted new males, indicating that females also have the power to minimize a new male's reproductive benefits. Yet, this has not been thoroughly studied. A final way through which females can mediate a new male's reproductive benefits is through dispersal. Females leaving their group after male 


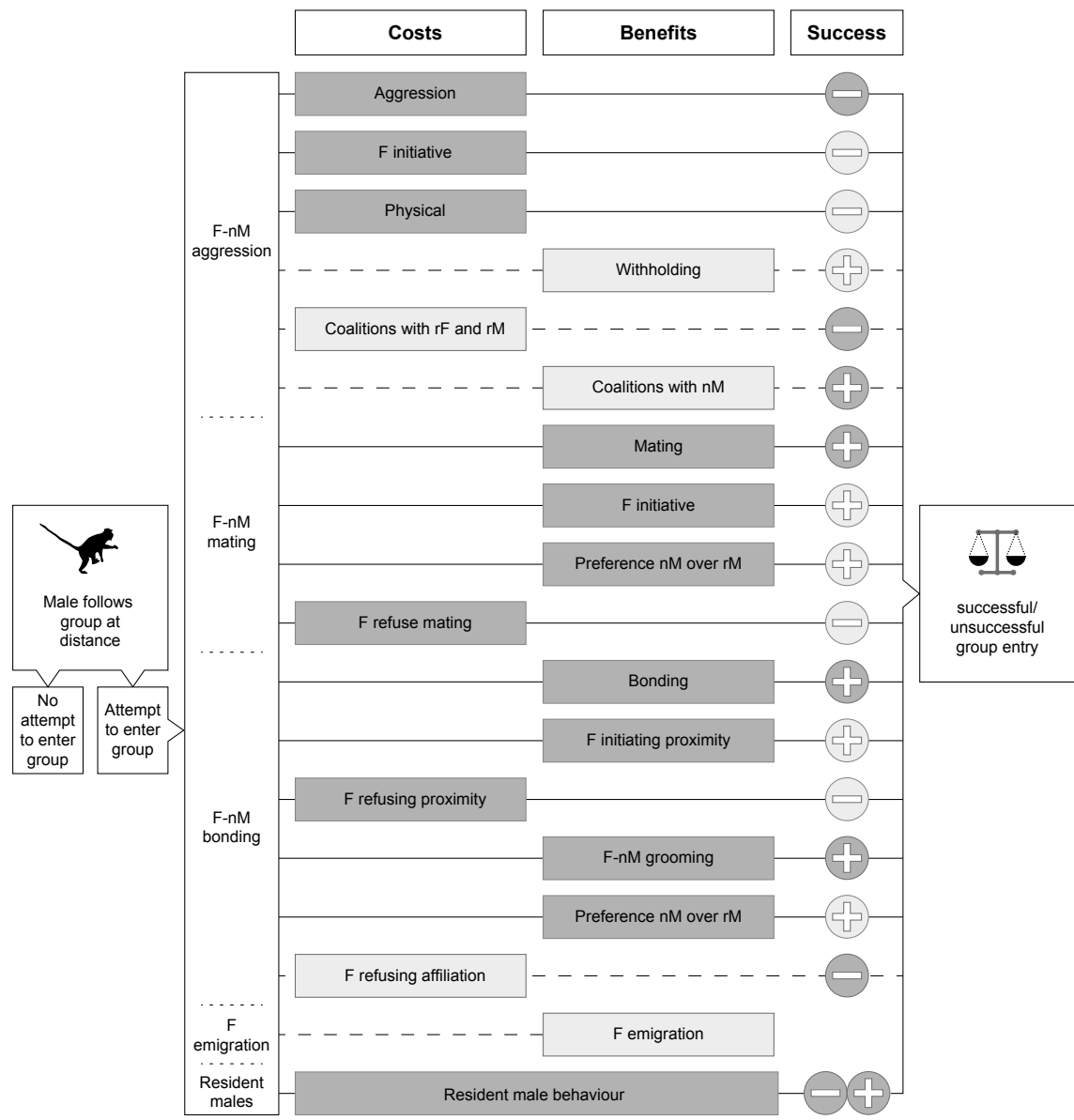

Figure 2 A schematic overview of the female influence on the process of male group entry. When a new male decides to enter, female behaviour can affect the costs and benefits they gain during group entry (Costs; Benefits). Moreover, female behaviour can affect a male's chances of successful group entry (Success). Costs and benefits are indicated by squares, the circles indicate whether the behaviour promotes (+) or prevents (-) success at entering a group. The black boxes and circles are effects that are supported by descriptions or measures found in the literature discussed in this review, while the grey boxes and circles indicate hypothesized

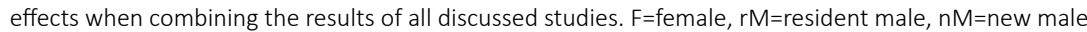


group entry will minimize a new male's reproductive opportunities. This has been reported a few times in literature, both in species with and without female philopatry. Yet, how common female dispersal after male group entry is remains to be established. Overall, it is evident that female sexual behaviour and dispersal can affect the benefits and success of male group entry.

In addition, males can benefit when females affiliate and form social bonds with them. Although studies reporting female-new male affiliation are limited, still an interesting pattern emerges when combining the little information present. Females can actively seek contact with new males, and commonly affiliate with them. They may even prefer new males as social partners. This way, females facilitate bonding with new males, which likely increases a male's chances of successful group entry. Yet, females may also prevent contact with new males through avoidance, or attack them when they come near. Infanticide risk may be an important factor mediating this response. Especially females at risk of infanticide may avoid new males as a counterstrategy (Hrdy 1977, 1979). Thus, female-new male affiliation can mediate a new male's benefits, and likely affect his chances of successful group entry.

Altogether, it is evident that females can affect the costs, benefits and success of male group entry. There may be several benefits for females affecting male group entry. First, they may protect themselves and their offspring against highly aggressive new males, thereby decreasing the risks of injuries and infanticide (Sterck and Korstjens 2000). This fits with the idea that female coalitions, which are likely necessary to prevent male group entry, are often formed in response to male harassment. Second, female mate choice may be important in determining the female's attitude towards new males. In species where females dispersal is common, females may leave their group when the resident male(s) are not preferred mating partners (Sterck and Korstjens 2000; Sterck et al. 2005; Qi et al. 2009). In other species, females may attempt to prevent group entry of unwanted males, while they may stimulate the entry of preferred males. Preferred males may not only be physically strong, but likely also have good social skills or are genetically interesting (Clutton-Brock and McAuliffe 2009). Thus, even though aggressing or associating with new, unfamiliar, males can be risky for females, they can benefit from actively influencing who is entering their group.

It is important to note that the data described in this review are likely biased for several reasons. First, studies focusing on female behaviour during male group entry are rare. Most information covered in this review is derived from male-oriented studies, where only a few sentences provide (non-quantitative) information on female behaviour. The presence of certain interactions may be mentioned, but the absence of behaviours is rarely reported. Therefore, it is difficult to estimate whether interactions between resident females and new males are not reported or not observed. Consequently, our review may underestimate or overestimate the role of female behaviour in male group entry. Second, not all female-new male interactions received equal attention in literature. Up to now, female-new male aggression and mating received considerable attention from researchers, which provides information about new males' costs and reproductive benefits. In contrast, information on bonding with 
new males and how females can promote successful group entry is too limited to draw hard conclusions. Lastly, the conclusions are based on information about a limited number of species. Only two species (i.e. Macaca mulatta and M. fuscata) have been extensively studied (Table 1 ). These species are closely related and have similar social characteristics (Thierry et al. 2000). This makes comparisons between all studied species, and identifying species characteristics affecting female behaviour during male group entry impossible. Therefore, more systematic studies focusing on female behaviour during male group entry in a wide variety of species are called for, describing the presence and absence of all possible interactions between resident females and new males.

However, it may take decades to collect sufficient data in the wild, as male group entry is unpredictable and may only occur relatively infrequent. In the meantime, captive studies on female behaviour during male introductions can be good starting point to gain better understanding of the consequences of female behaviour during male group entry. Within these future studies, there should be more attention for the consequences of female interactions with new males. The direct consequences of the female-new male interactions described in this review are often based on ideas and suggestions, yet they are barely ever measured. This is especially relevant when both resident males and females interact with new males, as is the case in multi-male multifemale primate groups. Likely, female behaviour affects resident male-new male interactions and vice versa (Yamada 1971; Winston 1985). Therefore, it is hard to disentangle what resident males and resident females contribute to the costs, benefits and success of male group entry. Here also, studies of male introductions in captive groups with no or few resident males may provide valuable information. Only if we understand how females behave during male group entry, why they do so, and when the consequences of female interactions with new males are clear, can we truly understand the fitness consequences of male dispersal for both males and females. Moreover, not studying female behaviour during male group entry ignores that females may actively influence who is entering their group.

\section{Conclusion}

To conclude, there is emerging evidence that female behaviour plays a key role in determining the costs, benefits and success of male group entry in primate species living in multi-male multi-female groups. Yet, information is only available on a limited number of species and this information is largely descriptive, which makes it difficult to generalize the results and identify the factors affecting the female influence on male group entry. In particular, more studies on positive social interactions between females and new males, and the consequences of these interactions are needed. However, it is clear that female behaviour should not be overlooked when studying male group entry. Females are active contributors to, and not passive onlookers in, the process of male group entry. 



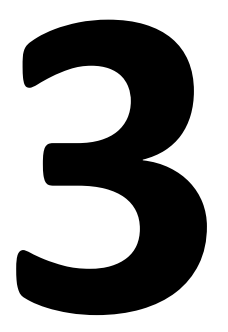

\section{The male introduction procedure for macaques at the Biomedical Primate Research Centre}

Astrid Rox, André H van Vliet, Jan AM Langermans,

Elisabeth HM Sterck, Annet L Louwerse 


\begin{abstract}
Male introductions into captive primate breeding groups can be risky and unsuccessful. Yet, they are necessary to prevent inbreeding in naturalistic breeding groups, while housing primates in such groups increases animal welfare. The procedure used to introduce new individuals may affect the risks and success of an introduction. At the Biomedical Primate Research Centre (BPRC) in Rijswijk, the Netherlands, male rhesus macaque (Macaca Mulatta) introductions into naturalistic social groups achieve relatively high success rates. This paper describes the male introduction procedure used at the BPRC. During introductions, males are stepwise familiarized with and introduced to their new group, while all interactions between the new male and the resident females are closely monitored. Monitoring the behaviour of the resident females and their new male during all stages of the introduction will provide crucial information on whether or not it is safe to proceed. The BPRC introduction procedure is widely applicable and may improve the management of captive primate groups in any housing facility world-wide. Thus, careful introduction management can minimize the risk associated with male introductions and enhance the welfare of captive primates.
\end{abstract}

\title{
Introduction
}

Animals play an important role in the life of many people. Animals are important companions, but are also often used for education and conservation in zoos, or for biomedical research. The welfare of all animals should be of major concern for everyone involved. Animal welfare should be of particular interest to biomedical researchers involved in animal testing, as impaired animal welfare is associated with physiological and behavioural changes that may affect the outcome of their studies (e.g. Barnett 1976; Mineka and Suomi 1978; Kempes et al. 2008; Taylor 2010). Improving animal welfare in biomedical research facilities may therefore result in the reduction and refinement of animal experimentation (Russel and Burch 1959).

An important marker of animal welfare, commonly used by researchers assessing the animal's quality of life, is the expression of natural species-typical behaviour (e.g. Bracke and Hopster 2006; Olsson and Westlund 2007; Fraser 2008). In particular, the expression of natural social behaviour is important for animals living in social groups, such as primates. Primates live in complex social groups, and have the behavioural need to engage in complex social interactions. The welfare of captive primates is impaired when they are restricted in displaying social behaviour, while primate welfare can be enhanced when they have the opportunity to engage in complex social interactions, similar to the interactions observed in wild primate groups (e.g. Rennie and Buchanan-Smith 2006). Therefore, it is important to house primates socially, preferably in naturalistic social groups where they can display natural social behaviour.

Many research institutes world-wide still house their primates under non-naturalistic circumstances, with group composition, demography and migration patterns differing from wild groups. In contrast, the primates in the breeding colony of the Biomedical Primate Research Centre (BPRC), mainly macaques (Macaca spp.), are housed in large 
naturalistic social groups. The composition, demography and migration patterns within these groups closely resemble nature to stimulate the expression of natural complex social interactions. Generally, wild macaques live in multi-male multi-female groups (Cords 2012), but also one-male groups have been observed in the wild (Neville 1968; Lindburg 1969; van Noordwijk and van Schaik 1985; Ménard and Vallet 1993; Keane et al. 1997; Singh et al. 2006). Similarly, the groups at the BPRC consist of multiple adult females, their dependent offspring, and one adult breeding male. Females are philopatric and remain in their natal group throughout their lives, similar to wild groups (Boelkins and Wilson 1972; Drickamer and Vessey 1973; Dittus 1975; Mehlman 1986; Sprague 1992; van Noordwijk and van Schaik 2000; Hayakawa and Soltis 2011). Consequently, these captive females form matrilines (i.e. families related in the female line), which are important for increasing group stability (McCowan et al. 2018; Rox et al. 2019). Males are removed from their natal group at puberty; the same age at which wild macaque males would leave their natal group (Boelkins and Wilson 1972; Drickamer and Vessey 1973; Sprague 1992; Mehlman et al. 1995; Sprague et al. 1998; van Noordwijk and van Schaik 2000). However, when female offspring remains in their natal group, the risk of inbreeding between a breeding male and his maturing daughters arises. Therefore, breeding males need to be replaced every three to five years to prevent inbreeding with maturing daughters, similar to wild male macaques, who change breeding groups several times in their lives (Boelkins and Wilson 1972; Dittus 1975; Sprague 1992; van Noordwijk and van Schaik 2000).

The introduction of a new breeding male into an established social group may be risky. New males may be rejected by the females and introductions may be unsuccessful (Bernstein et al. 1977; Rox et al. 2019). In extreme cases, females may injure or even kill new males (Rox et al. 2019). In addition, also females and their offspring are at risk during male introduction. Females may become injured in fights with new males (Cooper et al. 2001; Rox et al. 2019), and new males may kill dependent offspring (e.g. Zaunmair et al. 2015). Moreover, both new males and resident females may experience high stress levels during introductions, similar to male group entry in the wild (Alberts et al. 1992; Beehner et al. 2005; Marty et al. 2017b). Thus, male introductions may lead to stress, injuries or even death. Therefore, it is important to consider all different management strategies to prevent inbreeding in captive primate groups. An alternative way to prevent inbreeding in captive breeding groups is through the removal of all offspring before maturation. The current age-norm for removing macaque offspring for their natal group in is between 10-14 months (Prescott et al. 2012). Many research institutes adhere to this age norm and rear their macaques in peer-groups. Yet, peerreared individuals experience high levels of stress early in life, which has long-term consequences on their behavioural and physiological development and may affect biomedical research results later in life (Barnett 1976; Mineka and Suomi 1978; Kempes et al. 2008; Veenema 2009; Taylor 2010). Moreover, peer-reared individuals are often socially incompetent and form less stable groups (Kempes et al. 2008; Oates-O'Brien et al. 2010; Rox et al. 2019). This shows that removing offspring from their natal group before reaching adulthood compromises animal welfare, is not a good way to prevent 
inbreeding in primate groups. Therefore, naturalistic group housing with necessary male introductions can be considered a viable option to prevent inbreeding In captive primate groups.

The risks associated with male introductions may be minimized when the introductions are carefully planned and managed. At the BPRC, approximately $77 \%$ of the male introductions are successful, while $77 \%$ of the successful introductions lead to long-term stable groups (i.e. males can reside in the group until they are removed for management reasons (e.g. risk of breeding): Rox et al. 2019). These success rates are comparable to the success rates observed after pair formation in rhesus macaques (e.g. Reinhardt et al. 1995; Truelove et al. 2017); a far less socially complex situation. This implies that the success rate of male introductions at the BPRC is relatively high. Studies focussing on pair formation or the formation of new, non-naturalistic, social groups show that the success of an introduction may depend on the procedure used to compose the group (Bernstein 1969, 1971; Truelove et al. 2017). However, there is little information present in literature regarding the introductions of new males into established breeding groups, and (successful) introduction procedures have not been described or compared. Sharing the BPRC introduction procedure may therefore help other facilities that house captive macaque breeding groups, including zoos and research institutes, to improve their introduction management strategy. Moreover, it allows the comparison of male introduction methods and the search for an optimal introduction procedure. Thereby, the risks associated with male introductions cam be diminished while enhancing animal welfare.

\section{Methods}

\section{Housing conditions}

The BPRC houses two macaque species, rhesus macaques (Macaca Mulatta) and long-tailed macaques ( $M$. fascicularis). The housing conditions of the animals at the BPRC are designed to stimulate natural behaviour and optimize animal welfare. All macaques in the BPRC breeding colony are group-housed in spacious inside $\left(72 \mathrm{~m}^{2}\right.$, $2.85 \mathrm{~m}$ high) and outside enclosures ( $208 \mathrm{~m}^{2}, 3.1 \mathrm{~m}$ high). In total, the BPRC (in summer 2019) houses 28 breeding groups of rhesus macaques and 12 breeding groups of longtailed macaques. Animals have the opportunity to move freely between their inside and outside enclosure, both during day and night. Both enclosures contain a variety of environmental enrichment items, such as elevated beams, fire hoses, climbing structures and a pool, to stimulate natural behaviour (Vernes and Louwerse 2010).

Both the inside and outside enclosures contain different compartments. The inside enclosure consists of four different compartments, of which some are separated through wired mesh, and others are separated through concrete walls (Figure 1). The outside enclosure consists of three different compartments, all separated through wired mesh. The different compartment always have two or more connections to the other compartments, preventing high ranked group members from monopolizing access to certain compartments. These different compartments are used to introduce 
a new male into the group stepwise, and allows animal caretakers to regulate a new male's access to the group during the introductions.

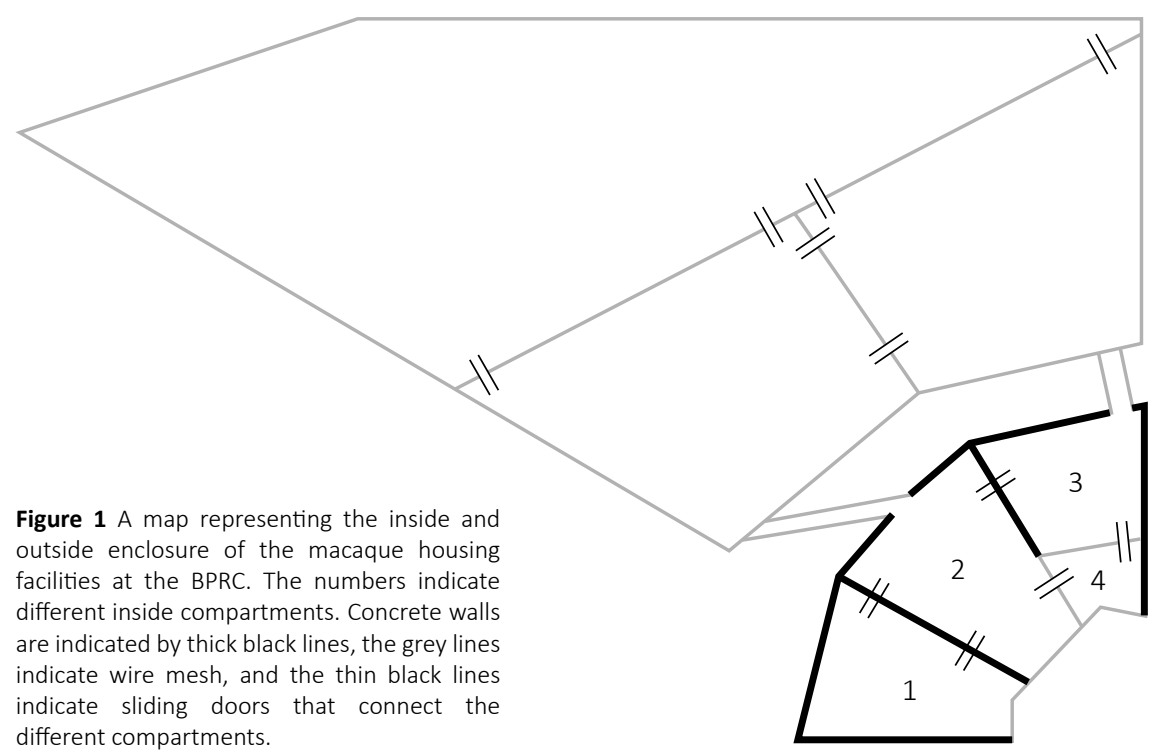

\section{Developing the BPRC introduction procedure}

The BPRC introduction procedure is developed based on personal knowledge and experience of the authors, in particular AHVV and ALL. As there is only limited information on male introductions into breeding groups available in literature, this personal knowledge is used in this paper to explain why particular decisions during the introduction process are made. We will refer to this information as personal communication. Whenever scientific studies are available to support the information provided, we will refer to these studies.

\section{Conducting male introductions at the BPRC}

At the BPRC, adult breeding males are removed from their group when the risk of inbreeding with adult daughters arises. It may take up to a year before a new breeding male is introduced after the removal of the previous male. A new male is introduced according to the BPRC introduction guidelines, wherein males are familiarized with and introduced into a new group stepwise. During the introductions, the new males need to obtain the alpha position immediately after group entry, mimicking bluff immigration in the wild (van Noordwijk and van Schaik 1985). The process of introducing a new breeding male is described below. When describing the procedure, we focus on five 
different steps, all crucial to optimize introduction success and long-term group stability, namely: 1) Selecting a breeding male; 2) Preparing the male and the group for the introduction; 3) Familiarizing the new male and the group; 4) Physically introducing the new male; and 5) Post-introduction monitoring.

The introductions are carried out by specialized animal caretakers, and are under close observation by behavioural specialists. The caretakers decide on the exact duration of the different phases of the introduction, depending on the specific behaviour of the animals involved. The introduction process described below particularly focuses on the process of introducing a new male to a rhesus macaque breeding group, as this species is most prevalent among research institutes. Yet the same procedure is used to introduce long-tailed macaque males at the BPRC. However, the timing of the introductions differs between the species. Rhesus macaque introductions take place between October and March, which corresponds to their breeding season, and is the natural time frame for male dispersal is this species (Boelkins and Wilson 1972; Drickamer and Vessey 1973; Bernstein et al. 1977). Longtailed macaque introductions take place throughout the year, as long-tailed macaques are non-seasonal breeders where male dispersal occurs throughout the year (van Noordwijk and van Schaik 1985, 2000).

\section{Male introduction procedure}

\section{Step 1. Selecting a breeding male}

Matching the right male to the right group is the first crucial step in increasing male introduction success. Biomedical research facilities often have multiple breeding groups and multiple breeding males available. At the BPRC, males are selected based on several parameters, of which genetics is one of the most important factors (step $1 \mathrm{~A}$ in Figure 2). It is key to maintain genetic diversity in captive populations and minimize inbreeding (Ballou et al. 2010). Males that are genetically overrepresented in the colony are excluded from introductions, while males with rare genes are particularly preferred breeding males. Moreover, male genetics should be dissimilar from the resident females' genetics (Ballou et al. 2010). This may also contribute to successful introductions, as females generally prefer genetically dissimilar males over males with genetics more similar to them (e.g. Setchell and Huchard 2010).

After genetic matches are made, social behaviour and social experience are assessed (step 1B in Figure 2). First, it is important that the new male is reared in a social group and stayed in his natal group for least 3.5 years to ensure proper social development (Rox et al. 2019). Moreover, the selected male should not have caused any social problems in his natal group (e.g. inappropriate aggression towards other animals). After removal from his natal group and transfer to an all-male group, he should not be involved in extensive aggression and not become injured or injure other males in his group. Moreover, he should not be afraid of other animals or humans, engage in positive social interactions with his group members, actively participate in positive reinforcement training, but not be too bold or too pronounced. Usually, these males 


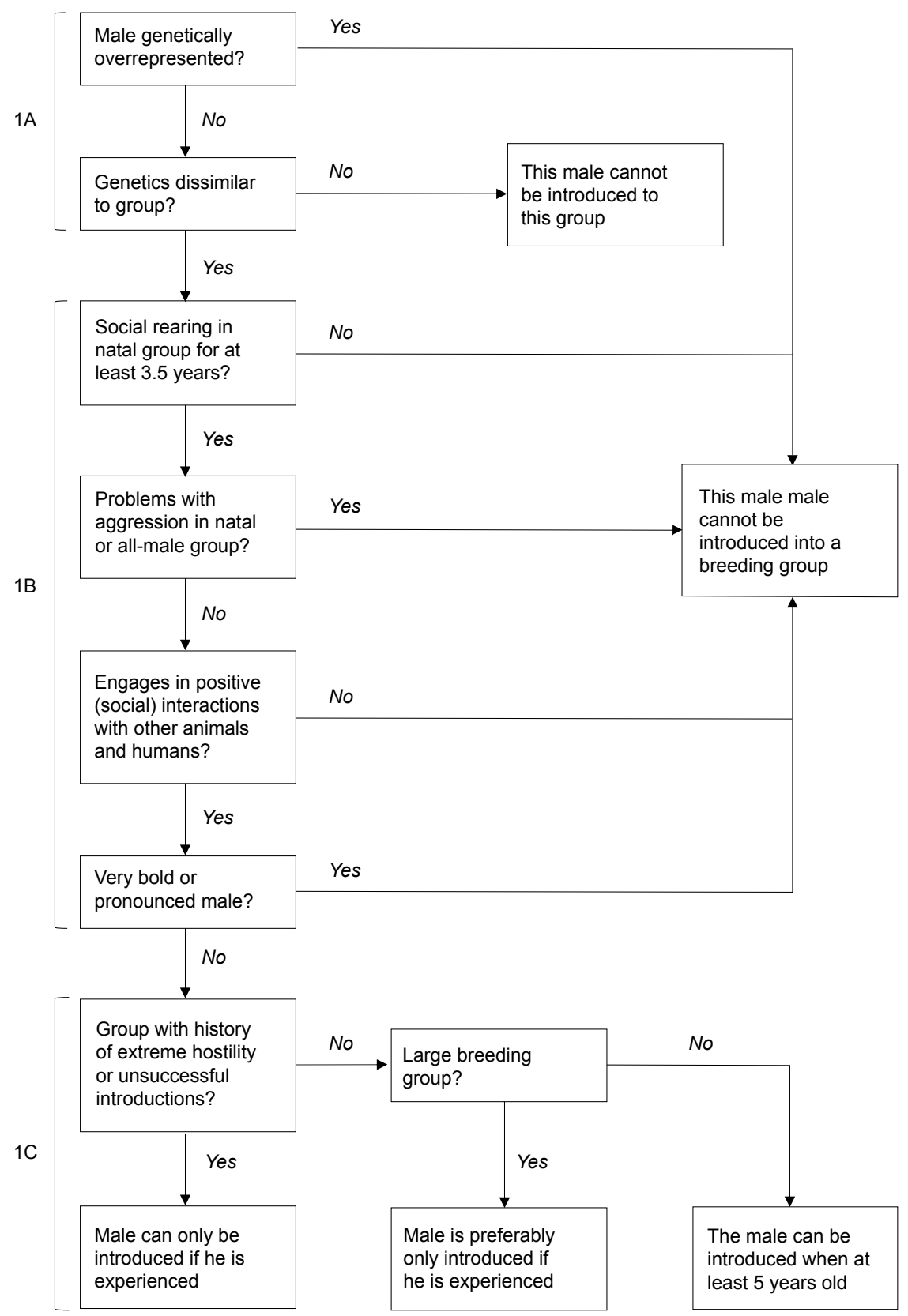

Figure 2 Step 1: The stepwise assessment to determine whether a male can be introduced into a particular group, concerning the new male's genetic (1A), his social behaviour (1B), and body condition (1C). 
turn out the be males from middle-ranking matrilines in their natal group (personal communication).

Finally, age and body condition are used to decide which of the candidate males will be introduced to the group (step $1 \mathrm{C}$ in Figure 2). Within the all-male groups, males are housed in after removal from their natal group, they have the opportunity to mature and grow to prime body condition. They can be introduced to a breeding group from the age of 5, after obtaining adult male body features. These young and inexperienced breeding males are usually introduced into smaller breeding groups to increase the chances of successful introductions (personal communication). Similarly, inexperienced and young wild long-tailed macaque males are more successful in obtaining the alpha position in smaller groups (van Noordwijk and van Schaik 1985). After they gained experience in their first breeding group and reached prime age (i.e. between 9 and 12 Rox et al. 2019), males may be introduced into larger and more complex social groups. These strong prime aged males are usually more successful during introductions (Marty et al. 2017a; Rox et al. 2019). In particular, young inexperienced males are never introduced into groups with a history of extreme hostility against new males and unsuccessful male introductions.

\section{Step 2. Preparing the male and the group for the introduction}

When a male is selected for introduction into a particular breeding group, the introduction needs to be prepared. It is key to carefully plan and execute the introduction to increase the chances of success.

First, the residing alpha male needs to be removed from the group, possibly alongside the sub-adult natal males (i.e. males aged 3 or more) (step $2 \mathrm{~A}$ in Figure 3). The males should be removed before the start of the breeding season to prevent female pregnancy. The presence of pregnant females decreases the chances of successful male introductions (Rox et al. 2019). At the BPRC, residing males are preferably removed from the group at the start of summer, in June or July. Groups always spend at least four weeks without the previous breeding male in their group before a new male is introduced. This allows the group to settle in their new situation and stabilize without an adult male present in their group (personal communication). However, removal of natal males is not necessary when a new male is introduced into the group right before or early in the breeding season, which corresponds with the natural timing of male group entry in the wild (Boelkins and Wilson 1972; Drickamer and Vessey 1973; Bernstein et al. 1977). At the BPRC, natal males may breed with their (half)sisters or other (related) females in their group from the age of 2.5 (unpublished data). The presence of natal males does not affect introduction success (Rox et al. 2019), and introducing a new male early in the breeding season may prevent the subordinate natal males from reproducing. However, when a group spends one or two breeding seasons without a breeding male, removal of natal males is necessary to prevent inbreeding. Introductions into groups that spend at least one breeding season without an adult male may be beneficial, as there are no infants present in these groups. This may remove a trigger for escalating female-new male aggression, which often starts when infants squeak or scream towards 


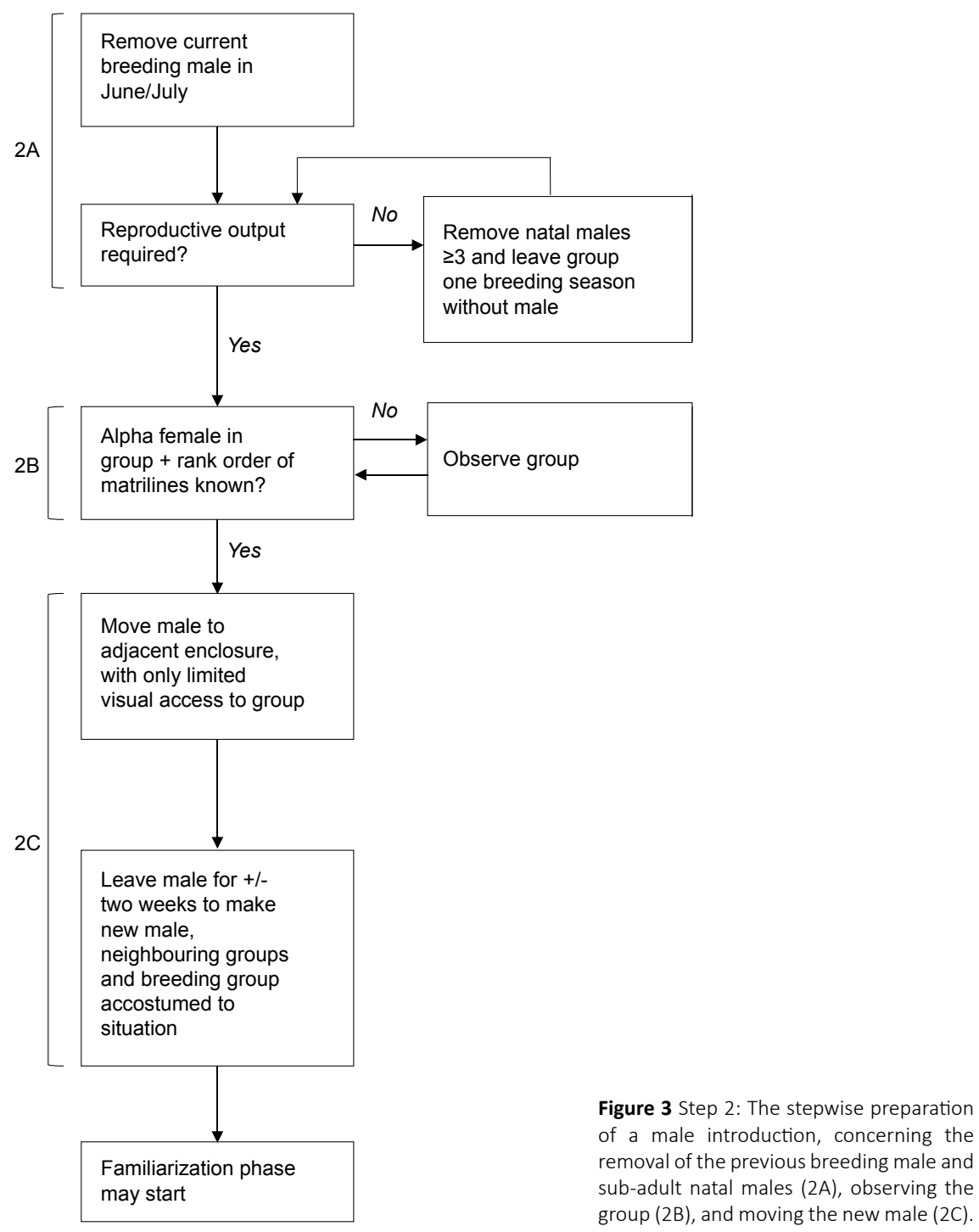

new males (personal communication). Bernstein et al. reported that all male introductions into groups of lactating mothers outside the breeding season were unsuccessful (Bernstein et al. 1977). However, data collected during 64 introductions at the BPRC rhesus macaque breeding colony shows that the presence of infants does not affect introduction success (Rox et al. 2019). Therefore, when high reproductive output is needed in the breeding colony, a new male can be introduced in the breeding season 
immediately following the removal of the previous breeding male. In summary, the resident breeding male should be removed before the start of the breeding season. Natal males may stay in their group if a new male is introduced in the same breeding season, but not when the group spends one or more breeding seasons without an adult breeding male.

Second, it is important to observe the group before a new male is introduced (step $2 \mathrm{~B}$ in Figure 3). The females that may have a large influence on the group's behaviour, such as the alpha female and the matriarchs, need to be identified. Moreover, the rank order of the different matrilines in the group needs to be known, and it needs to be clear which female belongs to which matriline. This information is crucial during the introduction, as this may help to predict whether fights between females and a new male are likely to escalate (personal communication). Females within the same matriline are likely to support each other in conflicts (Silk 2002; Suomi 2005; Silk et al. 2006; Oates-O'Brien et al. 2010). Moreover, it is necessary to understand the matrilineal group structure when the introduction goes wrong and the group needs to be split for some reason (e.g. a matrilineal overthrow due to social instability, or certain females that continue to aggress their new male). When the group structure is known, the group can be split relatively quickly while leaving the natural matrilineal structure of the group intact. It is important to conduct the observations after the previous breeding male and the sub-adult natal males are removed and the females of the group have settled in their new situation, as the presence of the males may affect female behaviour.

Third, once the group structure is known and the important females have been identified, the new male can be moved to an enclosure adjacent to the females (compartment 1 in Figure 1, step 2C in Figure 3). In this housing condition, the male is separated from his future group through a concrete wall and is housed solitary. This situation allows auditory, olfactory and limited visual contact between the new male, the group, and their neighbours. Spending some time solitary will allow the male to become acquainted to his new surroundings, in particular to the neighbouring groups, and recover from any potential stress associated with moving (see Davenport et al. 2006). This minimizes the chances of neighbouring groups interfering with the introduction (personal communication). Moreover, it is crucial for a male to understand that he is removed from his previous group and will not gain any support from previous group members during the introduction. This may prevent escalated aggression from the new male to the members of his new group (personal communication). At the $\mathrm{BPRC}$, the male is housed adjacent to the females for approximately two weeks before the introduction proceeds. The importance of the new male and the neighbours becoming acquainted to the new situation always needs to be balanced against the male being housed solitary, which may also impair his welfare. Therefore, it is important the introduction proceeds as soon as the new male and the neighbours are accustomed to the new situation. 


\section{Step 3. Familiarizing the new male and the group}

In the wild, new males may follow and observe groups from a distance before they attempt to enter or take over the group (van Noordwijk and van Schaik 2000). During this period, males may become gradually acquainted with the group and estimate the chances of successful group entry. A similar procedure, wherein a male is familiarized with his new group stepwise before physically introducing the male to the group (step 4 ), is used during male introductions at the BPRC. During the familiarization phase, the male is only able to interact with the group through wired mesh, and is not yet physically introduced to the group.

The first step of the familiarization phase is moving the male to another compartment of the inside enclosure where he can see the group through wired mesh, but not yet touch them (compartment 3 in Figure 1, step 3A in Figure 4). During this phase, the group has access to their entire outside enclosure, and two inside compartments (compartments 1 and 2 in Figure 1). The male will remain in this compartment until the first aggression between him and the group diminishes, which usually occurs after one or two days (personal communication). Next, the male will get additional access to a compartment where he can physically interact with the group through wire mesh (compartment 4 in Figure 1, step 3B in Figure 4). Interaction through the mesh is always supervised and is only allowed for a limited period of time each day. Generally, there are high levels of aggression between the resident females and the new male during the first interactions through the fence (personal communication). Therefore, interaction is only allowed for up to one hour on the first day. After this hour, the male is moved back to the compartment 3 (Figure 1), where he can see but not touch the females. On the second and third day, aggression levels usually decrease drastically and the first females may show interest in the new male and display affiliative or sexual behaviour through the wired mesh (personal communication). If aggression levels between the females and the new male remain high for several days and there are no signs of diminishing aggression, the introduction is stopped. This is particularly important when there is a risk of severe injuries on fingers, arms, and sometimes the face through the wired mesh. Additionally, the introduction is stopped when the new male displays submission to any resident group members, the male is not interested in interacting with the females at all and stays away from them for several days, or when females do not show any positive interest (i.e. display mating or affiliation) in the new male. When particular females remain highly aggressive to the new male, or start the majority of conflicts with the new male, these females may be individually introduced to the new male. In this case, the aggressive female is separated from the group and physically introduced to the new male in the inside enclosure, while the group is locked outside without visual access to the introduced pair. This visual barrier prevents the group from interfering in interactions between the new male and the female. Females may spend up to two hours with the new male. Individual females should not be separated from the group for too long (at most 4 hours), as this may lead to social instability and matrilineal overthrow within the group (unpublished data; OatesO’Brien et al. 2010). Usually, aggression levels diminish after this one-to-one contact 


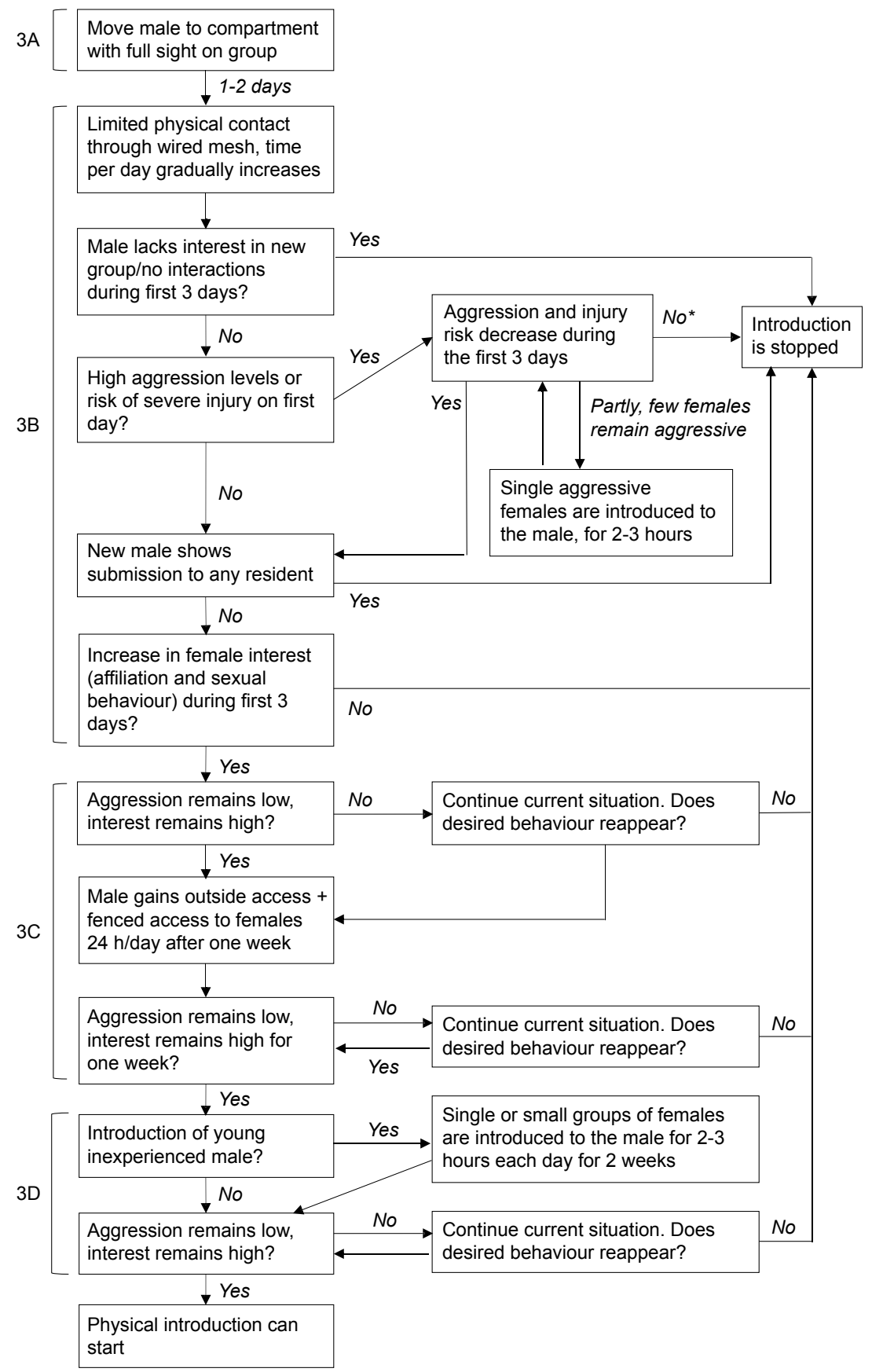


between highly aggressive females and their new male (personal communication). If one or a few females remain highly aggressive towards the new male even after oneto-one contact, the introduction of this male cannot continue in this group. Often, the introduction is stopped and considered unsuccessful.

However, sometimes, the female(s) aggressing the new male during an introduction have a history of repeated extreme aggression towards new males, and their behaviour has led to several unsuccessful introductions. In this case, one could consider permanently removing the repeatedly aggressive female(s) from the group. Yet, removal of individual females from a group may cause social instability (Oates-O'Brien et al. 2010), and can only be done when the remaining members of the female's matriline will be able to defend their rank without the presence of the removed female. Moreover, the welfare of the removed female will be impaired, as she will experience stress from moving (Davenport et al. 2006) and will be alone after removal from her group. Therefore, this measure should only be taken in extreme circumstances, and requires careful deliberation about all possible options. An alternative strategy may be removing the entire matriline of the highly aggressive female(s) from the group, forming two different breeding groups. This leaves the matrilineal structure of the groups intact, which increases social stability (Oates-O'Brien et al. 2010; Rox et al. 2019). Yet, this may not always be possible due to restrictions in space.

When female-new male aggression decreased and female interest increased during the first three days of contact though the fence and the situation remains stable for at least one week, the introduction can proceed. The male will gain additional access to one outside compartment, allowing him to interact with the female through the fence in both the inside and outside enclosure. Moreover, he will have access to these compartments separating him and the females through wired mesh for $24 \mathrm{~h} /$ day (step $3 \mathrm{C}$ in Figure 4). Access to the outside compartment is important as this may promote exercise. At the BPRC, animals are more active outside than inside (unpublished data). Outside access may therefore promote muscle formation, which in its turn, may increase introduction success (Rox et al. 2019). Moreover, males that are more fit will be better able to escape coalitionary attacks by females (personal communication). Therefore, it is important to ensure the new male is in good physical shape before he is physical introduced to his group. This situation will continue for one to two weeks.

The final step of the familiarization phase depends on the new male's social history: whether he is inexperienced or experienced. If the male is young and inexperienced, he will be physically introduced to small sub-groups of females each day for approximately two weeks (step 3D in Figure 4). The females will spend up to two hours

Figure 4 Step 3: The steps used to familiarize a new male and a breeding group, concerning providing full visual contact (3A), the first days of physical contact through wired mesh (3B), providing the male access to an outside compartment with limited supervision (3C), and introducing individual females to inexperienced males (3D). *When the introduction cannot continue in the group because of one or a few highly aggressive females who have a history of extreme hostility towards new males, an alternative strategy could be to remove the aggressive female(s) and continue the introduction. 
per day with the male in his compartments of the inside enclosure (compartments 3 and 4 in Figure 1), while the remainder of the group can see them through wired mesh (access to compartments 1 and 2, and their outside enclosure). During the remainder of the time, the male is able to interact with the entire group through wired mesh. Again, is it important to only separate females from their group for a limited amount of time each day to prevent social instability. This full physical access to part of the females may help an inexperienced male to practice his social skills and mating behaviour as an alpha male (personal communication). After two weeks of various small sub-groups of females visiting the new male the physical introduction may start. If the new male is an experienced breeding male, this final step may be skipped and the physical introduction may start after the male had fenced access to the females $24 \mathrm{~h}$ /day for one to two weeks. Note that if aggression between the females and their new male flares up again at any time during the familiarization phase, the introduction is slowed down to see whether aggression decreases again. If this is not the case, the introduction can be stopped at any time during this process.

\section{Step 4. Physically introducing the new male}

If the familiarization phase is successful, the male is physically introduced to the group. As there are no other adult males in the group, the new male should become the alpha male immediately after physical introduction into the group. Therefore, the introduced males can be seen as bluff immigrants, who attempt to obtain the alpha position in the group right after group entry in the wild (van Noordwijk and van Schaik 1985).

The physical introduction of a male starts in the outside enclosure, which consists of three different compartments separated through wire mesh. To increase the chances of the male successfully escaping a potential coalitionary attack by his new group, he gets the opportunity to explore the outside enclosure before he is introduced to the group (step 4A in Figure 5). The group will be locked inside, while the male gets access to his inside compartment and the entire outside enclosure for up to two hours. Usually, this is done the day before the physical introduction starts. The physical introduction starts outside because the experienced animal caretakers conducting the introductions can oversee the entire outside enclosure at once, while it is not possible to oversee the entire enclosure when the animals have access to both their inside and outside enclosures. This overview is crucial at the start of the physical introduction to be able to identify which individuals cause problems, and quickly interfere with the introduction when necessary. Moreover, the presence of an animal caretaker may cause a restrain on aggression during the introduction, as animals may be less inclined to impulsively attack each other in the presence of people (personal communication). Therefore, locking animals outside may decrease the risks associated with male introductions and helps the animal caretakers to oversee the group's behaviour.

Next, on the day the male is physically introduced to the group for the first time (step 4B in Figure 5), the group will be locked outside, limiting their access to their two outside compartments only (see Figure 1). The male will be housed in the third outside 
compartment, to which he already had access during the familiarization phase, and is able to interact with the group through wire mesh. If aggression levels remain low, the sliding doors between the compartment will open. The male and the group will have access to the entire outside enclosure, for approximately 1 to 1.5 hours on the first day. During the remainder of the day, the male will be able to interact with the group through wired mesh from his two inside (compartment 3 and 4 in Figure 1) and one outside compartment. The time a male spends with the group each day is gradually increased when the introduction progresses. At first, the male is only allowed to interact with the group under supervision by the specialized animal caretakers. All interactions between him and his new group members are closely monitored. Some females may tolerate the new male in their group from the first day, while others are more hostile (Rox et al. 2018). Unwilling females may defend themselves when the new male approaches them, which may result in a coalitionary attack on the new male. Therefore, the male should wait for the females to approach him, and only take initiative for interactions after she approached him first (personal communication). If a male keeps on approaching the females who run away and avoid him, almost like the male is herding sheep, the introduction is stopped for the day. The male will be returned to his own compartments where he can interact with the group through, and introduced again the next day. If this behaviour continuous for several days, the introduction is stopped.

However, even when a new male waits for females to take initiative, high levels of aggression, female-new male submission and mating may be observed at the start of introductions, while affiliation is relatively rare. Over time, aggression and submission rates should decline, while affiliation increases (Bernstein et al. 1977; Rox et al. 2018). High levels of aggression at the start of the introduction are in principle not problematic, the male may even use female threats to confirm his dominant position and show his strength and social skills by responding with appropriate counter aggression (personal communication). Generally, there is no need to interfere with aggression and animals will be able to sort out their new social position by themselves (personal communication). Therefore, the animal caretakers only interfere with introductions when aggression is extreme, and there is risk of severe injury or social defeat. Socially defeated males crouch passively and cease resistance to group attack (Bernstein et al. 1983). When socially defeated males are likely to be killed by resident females when they are not removed from the group immediately (personal communication). Therefore, immediate intervention is necessary to prevent females from socially defeating the new male when they coalitionary attack a new male and he is expected to lose this conflict. In this case, the introduction is stopped and considered unsuccessful. Moreover, the male should never be injured during the introduction, but female injuries may occur at the start of the introduction. If the new male becomes injured, the introduction should be stopped right away to prevent females from winning conflicts from the new male (personal communication). Within three days, aggression levels should have decreased. If the group still responds highly aggressive to their new male, the introduction is stopped. If only one or a few females remain highly aggressive, these females may be 
Physical introduction outside,

time physical access increases

gradually

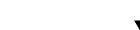

Male unpatient, follows avoiding females, "herding

sheep"

$\underset{N o}{\text { Yes }}$

Remove male for the day. few days?

New male shows submission to any resident

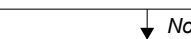

High aggression levels or risk of severe injury on first day?

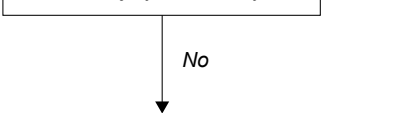

Male injured by females

$$
\checkmark \text { No }
$$

Male starts conflicts without clear trigger

$\downarrow$ No

Physical coalitionary attack by females, male expected to lose

$$
\downarrow \text { No }
$$

Several females tolerate new male in their group ${ }^{* *}+$ no

physical/serious conflicts for one week

\section{Yes}

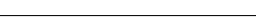

Aggression and injury risk decrea days

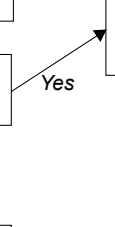

3

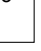
$+$

Partly, few females remain aggressive

Single females causing trouble are introduced to the male, for 2-3 hours
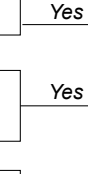

$\longrightarrow$ Yes $\longrightarrow \begin{aligned} & \text { Immediate } \\ & \text { intervention }\end{aligned}$

$\downarrow$ Yes

Supervision decreases,

caretaker moves to hearing

distance from group

$4 C$

\begin{tabular}{l|l}
\hline $\begin{array}{l}\text { No physical/serious conflicts for } \\
\text { several days, female interest } \\
\text { remains }\end{array}$ & \\
\hline
\end{tabular}

Continue current

situation. Does

desired behaviour remains

$$
\text { Yes }
$$

Continue current

situation. Does No

reappear?

Additional access to the inside enclosure is provided. Time of physical access gradually increases to $8 \mathrm{~h} /$ day, with minimal supervision.

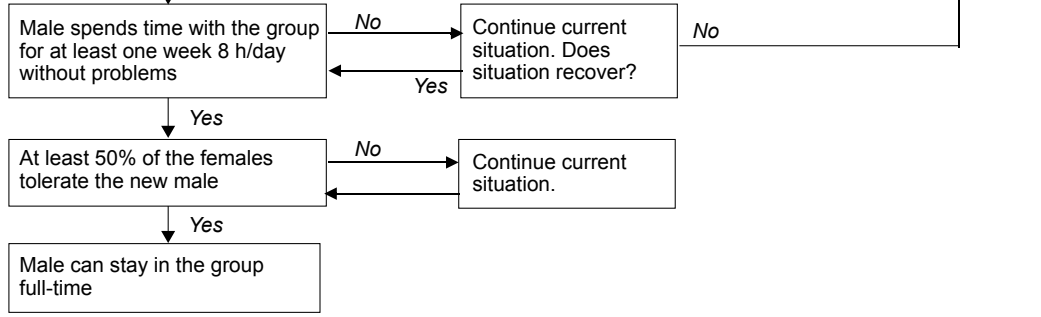


introduced to the new male alone, similar to the familiarization phase. In general, this decreases a female's hostile attitude. If not, one could consider stopping the introduction, removing the aggressive female(s) from the group, or splitting the group (see above). Moreover, the introduction should be stopped if the male shows submission to or loses conflicts from resident animals to prevent social defeat (personal communication). Finally, the introduction should be stopped if the male repeatedly responds with aggression to non-threatening social situations, as this shows social incompetence (Kempes et al. 2008). In summary, the first days of an introduction should be carefully monitored as the behaviour displayed during this period shows whether the introduction has potential to succeed or should be stopped.

The introduction progresses when several females tolerate the new male in their group (see Rox et al. 2018) and no physical or serious aggression has been observed for at least one week. First, the animal caretaker's supervision decreases (step 4C in Figure 5). The caretaker will move out of sight for some time when the male has physical access to the females, but will remain within hearing distance from the group. Consequently, the behaviour of the animals is no longer affected by the presence of people. Still, severe aggression will be noticed as this is generally accompanied by loud vocalisations (personal communication). Making use of video observations are helpful during this stage, as this will allow identification of the trigger and initiator of the conflict. If there is no increase in aggression or decrease in female interest in the new male, the animals will gain additional access to their inside enclosure whenever the male spends time with the group. Then, the time the male spends with the group gradually increases to approximately eight hours per day (i.e. a full workday) with minimal supervision (step 4D in Figure 5). When this goes well for at least one week, and at least half of the females in the group tolerated the new male and engage in positive social interactions (e.g. mating, proximity, or affiliation) with him, it is safe to leave the male in the group full-time (personal communication). It is not necessary for all female to tolerate the new male in their group for an introduction to be successful, as their relationship may grow after the introduction (Rox et al. 2018). Yet, the majority of the females need to tolerate the male, as this minimizes the risk of female coalitionary attacks on the new male (personal communication). The specialized animal caretakers make the decision to proceed with the introduction based on knowledge and experience. However, usually, when the male is completely settled in his new group, he becomes less cooperative with separation and returning to his own compartments at

\footnotetext{
Figure 5 Step 4: The stepwise physical introduction of a new male into a breeding group, concerning the male exploring the outside enclosure (4A), the first days of physical interactions outside (4B), decreasing direct supervision (4C), and proving additional access to the inside enclosure (4D). *When the introduction cannot continue in the group because of one or a few highly aggressive females who have a history of extreme hostility towards new males, an alternative strategy could be to remove the aggressive female(s) and continue the introduction. ${ }^{* *}$ for definition see Rox et al. 2018
} 
the end of the day. This is an additional sign that the male is ready to remain in the group full-time (personal communication). To increase introduction success, it is crucial to take steps slowly, and give the animals time to establish new social relationships (personal communication). Thereby, not only aggression but also the establishment of positive social relationships between the females and their new male should be considered.

\section{Step 5. Post introduction monitoring}

It is important to continue to monitor the group after the introduction has finished. Not all social relationships may have been established during the introduction. Moreover, during new contexts that the animals did not experience during the introduction, such as feeding or sleeping arrangements at night, may trigger aggression. Therefore, the group and the male should be checked immediately in the morning following his first night in the group. Hereafter, they are checked once a day, for at least 30 minutes per day, by the caretaker who conducted the introductions, and twice daily by the animal caretaker responsible for the daily care of the animals. During these checks, special attention is payed to possible injuries and other signs of social instability (e.g. increased stress-related behaviour or aggression). The introduction is considered successful when the male stayed with the group full-time for four weeks (Rox et al. 2018).

\section{Discussion}

This paper describes the procedure used to introduce an adult breeding male into a naturalistic group of rhesus macaques at the BPRC. Introducing new males is necessary to prevent inbreeding between maturing daughters and adult breeding males in naturalistic macaque groups. Yet, the process of male group entry may be risky, and male introductions may be unsuccessful. The success and risks associated with male introductions may be affected by the introductory procedure (Bernstein 1969, 1971; Truelove et al. 2017). To our knowledge, this paper is the first to describe a successful procedure to introduce an adult breeding male into a naturalistic group of rhesus macaques in detail. The male introduction procedure used at the BPRC shows relatively high success rates (Rox et al. 2019). Sharing this procedure may therefore help to improve male introduction management in other facilities that house captive macaque breeding groups.

Key to the BPRC introduction procedure is careful monitoring of the behaviour of the animals involved. Particular attention should be provided to the male's ability to win fights from female coalitions, as it is key the male obtains and maintains the dominant position in the group. Once a male loses a conflict, which may result in social defeat, he will not be able to obtain a dominant position in another group (personal communication). This is in line with the winner-loser effect, which is widespread throughout the animal kingdom and shows that once an individual loses a conflict, he is significantly less likely to win future conflicts (reviewed in: Rutte et al. 2006). Therefore, it is crucial to introduce a new male stepwise and the introductions are conducted by people that are experts in primate behaviour. At the BPRC, specialized 
animal caretakers conduct the introductions, in close collaboration with an ethologist. They attempt to minimize the risks of males losing conflicts from females by monitoring aggression, submission, and positive social interactions between females and their new male. Moreover, they take notice of who initiates conflicts and the triggers of aggression to identify individuals that are particular risk to the introduction. Whenever they are in doubt whether an introduction progresses well, they will not proceed to a next step of the introduction process, or even revert to an earlier step. Taking additional time to introduce a new male will not harm the introduction, introducing a male too fast can have detrimental consequences (personal communication). However, when an introduction takes too long, it is questionable whether the male and the group are a good match. In this case, stopping the introduction may be the preferred option.

We described the steps that can be taken during male introductions and identified potential behaviours signalling whether the introduction progresses well or not, but it is impossible to cover all interactions during male introductions. It is important to realize that every introduction is different, and there is not one perfect way to introduce a new male into a group. Small, unanticipated events, such as a squeaking baby, may already trigger a coalitionary attack on a new male, even when the introduction progressed well for several weeks (Rox et al. 2019). Therefore, flexibility in the process of introducing a new male is required, and the introduction should remain under close supervision.

There is only limited published information on male introduction management. The results of the few publications analysing the factors affecting the outcome of male introductions (e.g. (Bernstein et al. 1977; Rox et al. 2019) are generally in line with the BPRC introduction procedure, which strengthens the notion the BPRC introduction procedure is a relatively good and well-established method. Moreover, the success rate $(77 \%)$ is comparable to the success rate of the formation of pairs in rhesus macaques, a far less socially complex situation (Reinhardt et al. 1995; Truelove et al. 2017; Rox et al. 2019). Still, most of the procedure is designed based on experience and insights of the ethologists at BPRC. Up to now, there are no possibilities to compare the outcomes and guidelines of the BPRC introduction procedure to the procedures used at other facilities. Therefore, we call for more studies describing and analysing male introduction procedures. Only if we combine information from more institutes all over the world, we may find the best way to manage male introductions. In the meantime, the BPRC introduction procedure can be used to optimize the management of captive primate groups at other facilities.

The introduction procedure described in this paper focusses on the introduction of a new male into rhesus macaque groups in the breeding colony of the BPRC. The BPRC houses many different rhesus macaque groups, providing the luxurious position wherein a breeding male can be carefully matched with a new group. Yet, even when there is only one possible breeding male to a particular group, which may be the case in zoo-housed primates, the BPRC introduction procedure can still be used to introduce a new male to a group stepwise. The risks associated with male introductions may even be larger in this case, as the selected male may not meet all the criteria used to selected 
breeding males at the BPRC. Moreover, the BPRC introduction procedure may also be used to introduce new males into non-naturalistic primate groups. The risks of female coalitions will be lower in such groups, as there will be no matrilines that naturally support each other in the group (Silk 2002; Suomi 2005; Silk et al. 2006; Oates-O'Brien et al. 2010). Consequently, the chances of female socially defeating a new male will be lower. Yet, females may still exclude the male from their social unit and prevent successful introductions (Bernstein et al. 1977). Thus, also male introductions into non-naturalistic primate groups should be conducted carefully. Finally, the introduction procedure described in this paper can also be used to introduce new males into existing social groups in other primate species. The BPRC uses the same procedure to introduce new males in rhesus macaques and long-tailed macaques. Male introductions into long-tailed macaque groups may be expected to be more challenging, as this species breeds a-seasonally (van Noordwijk and van Schaik 2000). Consequently, male introductions into groups containing pregnant or lactating females, factors decreasing male introduction success in rhesus macaques (Bernstein et al. 1977; Rox et al. 2019), are more common in this species. Yet, almost $100 \%$ of the male introductions into long-tailed macaque groups are successful at the BPRC (unpublished data). This shows that the same procedure is successful in introducing males in different species, and possible success rates may differ between species.

In summary, the BPRC introduction guidelines are widely applicable, and can be used by any facility housing captive primates, including zoos, rescue or rehabilitation centres, and research facilities. Thereby, the risk associated with male introductions and the chances of unsuccessful male introductions can be minimized, while enhancing the management of captive primate groups and primate welfare. 
30 



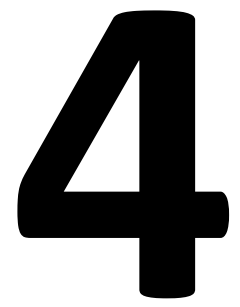

\section{Female social behaviour during three male introductions in captive groups of rhesus macaques}

Astrid Rox, Han de Vries, Annet L Louwerse, Elisabeth HM Sterck 


\begin{abstract}
Introductions of new males into captive primate groups are often necessary to prevent inbreeding, but also bear high social risks. To minimize these risks, it is crucial to understand the social behaviour accompanying male introductions. While the behaviour of new males is generally understood, information on resident female behaviour during introductions is lacking. We studied female behaviour towards the new male during introductions of three adult male rhesus macaques (Macaca mulatta)-each into a different captive group. All three males were successfully introduced; respectively $100 \%, 92 \%$, and $83 \%$ of the females tolerated the male as a group-member at the end of the introductions. Older females started tolerating the male significantly faster than younger females, while no additional effect of female dominance rank, fertility, or the number of female coalitionary partners on timing of tolerance was found. During the course of the integration, female aggression and submission towards the male, and male mating access decreased, while female affiliation towards the male increased. The increase of female tolerance and the changes in social behaviour were similar between the introductions, indicating a general pattern in female behaviour, although some variation in effect size and significance level was observed. Based on these results, we suggest that low female submission levels towards an introduced male may constitute a criterion to assess the risk of leaving the male in the group full-time. Moreover, low female aggression levels at the end of the introduction may signal long-term group stability. Overall, we conclude that female behaviour can provide valuable information about the male introduction process and should not be overlooked.
\end{abstract}

\title{
Introduction
}

Nowadays, naturalistic group housing is the preferred way to house captive social animals. Naturalistic group housing not only concerns a natural, or close to natural group composition, but also mimicking of group dynamics observed in the wild, such as migration patterns. In the wild, migration promotes gene flow between groups and prevents inbreeding (Moore and Ali 1984; Krause and Ruxton 2002). Similarly, in captivity inbreeding is often prevented by introducing unfamiliar animals into existing social groups. The introduction of unfamiliar animals is thus necessary for captive management, but also bears risks. The social risks of immigration found in wild animals may also be present during captive introductions mimicking the wild immigration process.

The risk of immigration of unfamiliar animals into a group is especially well reported in primates. Migration in primates is often male-biased (Greenwood 1980). Primate males face the challenge of immigrating into a social group, comprised of a cohesive core of related resident females, and obtaining a new position in the social network. This challenge is especially prominent in species that live in multi-male multi-female groups. The entrance of new males in such groups is associated with high levels of aggression, in both captive and wild situations (captivity: Rose et al. 1972; Bernstein et 
al. 1977, wild: Alberts et al. 1992; Zhao 1994; Bercovitch 1997; Teichroeb et al. 2011; Marty et al. 2017). Additionally, there is risk of injuries for adults of both sexes (wild: Packer 1979; Pereira 1983; Zhao 1994; Marty et al. 2017, free-ranging: Lindburg 1969), high stress levels (wild: Alberts et al. 1992; Marty et al. 2017) and low immune resistance (wild: Alberts et al. 1992). Moreover, new males can be infanticidal, posing a threat to resident females' young (captivity : Zaunmair et al. 2015, wild: Hrdy 1979; Pereira and Weiss 1991; Borries 1997; Sterck and Korstjens 2000; van Belle et al. 2010). Males can vary in their behaviour when entering a group; some males are aggressive while others may be more unobtrusive (wild: van Noordwijk and van Schaik 1985, 2000; Marty et al. 2016). The male's behavioural strategy may influence his success in establishing a position in the new group. A male may not be accepted by resident group members, and thereby not succeed in entering the group (captivity: Bernstein et al. 1977, free-ranging: Vessey 1971, wild: Yamada 1963, 1971; Neville 1968; Packer 1979; Packer and Pusey 1979; van Noordwijk and van Schaik 1985). These risks associated with the entrance unfamiliar primate males into existing social groups are well acknowledged, yet the behavioural process accompanying integration (i.e. establishing a social position) is understudied.

Our current knowledge on social behaviour during integration mainly derives from relatively old, often descriptive studies of both wild and captive primates, which focus on the new male's behaviour. These studies report that initial high levels of aggression decrease over time (captivity: Rose et al. 1972; Bernstein et al. 1977). After a brief period of aggression, new males can engage in grooming with resident females (captivity: Bernstein et al. 1977). Moreover, high levels of submissive behaviour commonly accompany interactions between unfamiliar animals (captivity: Bernstein and Mason 1963; Rose et al. 1972; Brent et al. 1997). Overall, these studies provide general understanding of the behaviour of new males. However, the role of resident females during male entrance has not received much attention, although their tolerance of the new male is probably crucial for successful integration (wild: Yamada 1971). The few studies addressing female behaviour during the entrance of a new male show contrasting results. Some females may not tolerate a new male and refrain from associating, while others respond with aggression (captivity: Kawai 1960; Rose et al. 1972; Bernstein et al. 1977). Females may employ aggression to prevent new males from entering a group through the formation of coalitions (wild: Packer and Pusey 1979). Female aggression towards new males may be absent in successful introductions (captivity: Zaunmair et al. 2015), but also when female aggression is present, it may quickly be replaced by affiliation (captivity: Bernstein et al. 1977). Moreover, females may engage in high levels of mating immediately after male entrance into a group, since females prefer to mate with novel males (captivity: Bernstein and Mason 1963; Rose et al. 1972, free-ranging: Manson 1994, 1995, wild: Packer 1979), and submission levels are high (captivity: Rose et al. 1972). However, no quantitative data on female behaviour towards new males are available, although their behaviour is probably directly linked to introduction success. In addition, female behaviour may differ 
depending on their individual characteristics. Younger females compared to older ones may be more afraid of the new male (Bernstein and Ehardt 1985). Furthermore, high ranking females may associate more often with the new male (Kawai 1960). In addition, females whose fertility occurs sooner after the start of the introduction may tolerate the new male sooner, since fertile females associate more often with unfamiliar males than non-fertile females (captivity: Zaunmair et al. 2015). Lastly, females forming larger coalitions may tolerate the male later, since their coalitions are stronger and may thus be better able to prevent male entrance. Moreover, females disliking the new male may be more likely to form coalitions. Altogether, it is crucial to increase our quantitative understanding of resident female behaviour during male introductions for the improvement of introduction management procedures.

This study has two aims: (1) to identify the female characteristics that influence female behaviour towards new males and (2) to provide a general understanding of female social behaviour during male introductions. To this end, female behaviour towards the new male was studied during introductions of three adult male rhesus macaques (Macaca mulatta)-each into a different captive social group consisting of adult females and their immature offspring. Though wild rhesus macaques generally live in multi-male multi-female groups, there is often only a single male present in captive groups. Creating single-male, multi-female groups prevents male-male competition, which bears high risks in a confined enclosure. In our study, single-male, multi-female groups were created, and natural migration patterns were mimicked.

During the introductions, we expect variance in female tolerance of the new male, possibly depending on individual female characteristics, namely her age, dominance rank, fertility, and on the number of her female coalitionary partners. In addition, we expect that female social behaviour towards the new male changes during the introductions, namely that initial high levels of female aggression, submission and mating towards the new male decrease and initial low levels of affiliation increase. Finally, we studied the new males' behaviour at the start (i.e. first five hours) of their introductions, since this may explain possible differences in female behaviour. This part of our study is by necessity explorative due to the limited number of introductions.

\section{Methods}

\section{Subjects and housing}

Subjects were 41 female rhesus macaques, ranging in age from 3 to 19 years, living in three different social groups (the Liby group, the Marieke group, and the Clio group). The groups consisted of 12 to 16 adult females ( $\geq 3$ years of age) and their non-adult offspring (Table A1-A3 in Appendix 1). Female offspring remain in the group during their whole life. Male offspring are removed around 4 years of age, mimicking natural migration processes.

The groups were housed at the Biomedical Primate Research Centre (BPRC) in Rijswijk, the Netherlands. The enclosures contained multiple elevated beams and environmental enrichment items (Vernes and Louwerse 2010). The inside enclosure 
measured $72 \mathrm{~m}^{2}$ and was $2.85 \mathrm{~m}$ high, the outside enclosure measured $208 \mathrm{~m}^{2}$ and was $3.1 \mathrm{~m}$ high. The three compartments of the outside enclosure were separated by wire mesh. Concrete walls separated inside compartments one, two, and three. Inside compartment four was separated from the compartments two and three by wire mesh (Figure A1 in Appendix 1). Outside introductory events, the resident group had access to inside compartments one and two and all three outside compartments and the new male was housed in inside compartments three and four. Animals received standard monkey chow [Ssniff ${ }^{\odot}$ ] in the morning and fresh fruit, vegetables or bread in the afternoon. Water was available ad libitum.

\section{Introduction process}

Three adult males were introduced during this study-each into a different social group. All three introductions took place during the breeding season and will hereafter be referred to as introduction A (Liby group), introduction B (Marieke group), and introduction $C$ (Clio group). The introductions were part of the regular management procedures at BPRC and followed the BPRC introduction guidelines. An experienced animal caretaker carried out the introductions, deciding on the duration of the different phases of an introduction by estimating the risks of severe attacks from the females to the new male and vice versa, based on personal knowledge and experience. Before the introduction, the residents and the new male were familiarized with each other, by allowing interaction through wire mesh. The male was then introduced to the group. During the introduction, contact possibilities between the resident females and the new male, and time of contact were progressively increased. At first, the animals only had access to the outside enclosure during introductory events. After four to seven days of contact, the animals were given additional access to the inside enclosure during introductory events. The duration of these introduction events began at 1 to 1.5 hours on the first day and was gradually increased to approximately 7 hours per day. The exact timing typically differed between introductions. Ultimately, the male was allowed to remain in the group full-time: this happened after 12, 24, and 20 days with physical contact for introductions $A, B$, and $C$, respectively.

The husbandry process of introducing a new male needs to be distinguished from the male's integration process. The introduction of an animal is a husbandry process. An introduction is considered successful when the new male is allowed to remain in the group full-time for at least 4 weeks (Brent et al. 1997). This criterion is based on introductions of chimpanzees (Pan troglodytes), with the aim of composing long-term stable groups, comparable to our study. Integration, in turn, concerns the process of establishing a social position in the group, and can be studied by looking at an animal's behaviour. While the introduction process is typical for captivity, the integration process takes place both in captive and wild groups. Whether a male successfully integrates into a group is based on female tolerance of the new male (see Measures below, based on: Bashaw et al., 2010; Meder, 1990). 


\section{Behavioural observations}

Data were collected between December 2014 and May 2015. Continuous focal observations of the new male and his female interaction partners were carried out during the study. We scored the social interactions among the new male and the females whenever the male was visible to the observer. All aggressive, unprovoked (i.e. not in response to aggression) submissive and affiliative behaviour from the females towards the male and vice versa was recorded. Furthermore, we scored all approaches from and towards the male, whether these were accepted (i.e. remain within $1 \mathrm{~m}$ for at least 3 seconds) or rejected (Kempes et al. 2008), and all mating events. Finally, submission, i.e. bared teeth and give ground behaviour between all group members was scored ad libitum. For a detailed description of the recorded behaviours, see the ethogram in Appendix 2.

Behavioural observations were conducted whenever the new males spent time with the group, on workdays between 9:00 $\mathrm{h}$ and 12:00 h, and between 13:00 h pm and 16:00 h. Observations started at the first day of physical contact and ended 2 (introductions $\mathrm{A}$ and $\mathrm{C}$ ) to 4.5 (introduction $\mathrm{B}$ ) weeks after the completion of the introduction (i.e. the male was allowed to remain in the group full time). In total, 48 (introduction A), 95 (introduction B), and 66 (introduction C) hours of observational data were collected, of which the male was visible for 24 (introduction A), 44 (introduction B), and 33 (introduction C) hours. A different observer observed each introduction. All observers were trained until they reached agreement with the first author. The location of the observations ( $50 \%$ inside and $50 \%$ outside) followed a semirandom schedule when the animals had inside and outside access.

\section{Fertility scoring}

Female sex skin coloration was used as a measure for fertility during introductions $A$ and $C$. The sex skin coloration of each female was scored once every observation day. Skin coloration was measured at three locations on the face and one location on the hindquarters (cf. Dubuc et al. 2009), using Pantone ${ }^{\odot}$ colour scales. Fertility scores ranging from 1 to 12 were assigned to the Pantone $C$ and PC colours 1767, 1777, 1787, Red 031, 1797, and 1807. The lightest colour received score 1 and the darkest colour received score 12 . Female sex skin coloration was assigned the same score as the Pantone colour it resembled most closely. The comparisons were made from a distance of approximately $5 \mathrm{~m}$. Visual comparisons are known to be equally reliable as the digital analysis of red, green, and blue reflectance values (Dubuc et al. 2009). All animals in a group were scored in the same area of the enclosure and under similar artificial lighting conditions. The observers were trained until they reached agreement with the first author in real life observations. Moreover, more than $80 \%$ agreement was reached in a picture test.

Monthly peaks in female sex skin coloration were only visible on the hindquarters and not on the face. Two females in introduction $\mathrm{C}$ showed no variation in sex skin coloration. 


\section{Measures}

Female tolerance of the new male occurred when he gained social access to her (based on: Bashaw et al., 2010; Meder, 1990). Female tolerance was considered to have been granted by a female when at least one of the following is true: 1) she accepted at least 3 of the last 4 approaches by the male; 2 ) she approached the male at least twice non-aggressively; 3 ) she had been together (i.e. within $1 \mathrm{~m}$ for at least 3 seconds) with the male at least twice outside the context of sexual encounters; or 4) she groomed, or was groomed by, the male at least once. For the analysis, intolerant females $(\mathrm{N}=3$ ) were considered to have tolerated the new male on the last observation day +1 day, based on the assumption that they would have tolerated the male later. In any event, the results were hardly affected when these three individuals were excluded from the analysis.

The female characteristics used in this study concerned four different individual measures. The first measure is female age on the first day the male was introduced. The second measure is female dominance rank in the social hierarchy which is based on bared teeth and give ground (Altmann 1962; de Vries 1998). The female dominance hierarchy was significantly linear in all three groups (Liby group: $h^{\prime}=0.96$; Marieke group: $h^{\prime}=0.85$; Clio group: $h^{\prime}=0.80$, all $p \leq 0.002 ; h^{\prime}$ is Landau's linearity index corrected for the number of unknown relationships (de Vries 1995)). The dominance rank was set on a scale with the most dominant female ranking 1 . The third measure is the first day a female achieved the highest fertility score on her hindquarters. We considered this to match her first peak in fertility during the introduction. Females not showing any variation in sex skin coloration $(\mathrm{N}=2)$ were considered to have their peak at the last observation day +1 day. In any event, the results were hardly affected when these two individuals were excluded from the analysis. The fourth individual measure is the number of female coalitionary partners that each female has; this was determined by the total number of different females aged $\geq 3$ years that supported the subject in conflicts with the new male. A female supported another female when she joined an already present conflict by displaying aggressive behaviour towards the male or his supporters.

The number of successful copulations between the females and the new male, regardless of female identity, was used as a measure of male mating access. Since female collaboration is required for mating, this is not only determined by the male. Consistent with this view, forced mating by the male was rare (5 out of 1661 copulations) and females could successfully reject male mating attempts (90 out of 1223 maleinitiated mating attempts) (cf. Overduin-De Vries et al. 2012).

\section{Data analysis}

To identify the new male's behavioural strategy, rates per hour of his aggression, submission, affiliation, and mating were calculated for the first 5 hours a male had physical access to the females (covering the first 4 (introduction A), 5 (introduction B), and 3 (introduction $C$ )) days of the introduction. We choose an equal period of time rather than an equal number of days so that all males had equal opportunities to 
interact with the females. A male's initial attitude towards the females was typically observable during this time frame. Note that our sample size of three males is too small to allow for a meaningful statistical analysis of male behaviour.

The focus of this study was on female social behaviour, which was analysed using the following approach. First, the day of tolerance was determined for each female. Next, general linear models, each including separate slopes for the three introductions, were fitted to test the effect of (1) female age, (2) the inverse of female age (1/age; this transformed variable is used to test for a non-linear (i.c. negative exponential) relationship between age and day of tolerance), (3) female dominance rank, (4) female fertility, and (5) the number of female coalitionary partners on the timing of female tolerance. In these models, the day of tolerance is the dependent variable, while the female characteristics are predictor variables, and the introduction $(A, B$, or $C)$ is entered as categorical factor in the models. We employed the following stepwise forward selection procedure to arrive at the best fitting model. We started by testing each of the five predictor variables (age, inverse age, female rank, female fertility and number of coalitionary partners) separately, to see which of these predictors explained the highest percentage of variance $\left(\mathrm{R}^{2}\right)$ in the dependent variable: 'day of tolerance'. This turned out to be the variable 'inverse age'. In the next step, we added each of the other four variables (age, rank, fertility, and coalitionary partners) separately, and used $F$ tests to see whether each of these variables would add significantly in explaining the variance $\left(R^{2}\right)$ in 'day of tolerance'. It was found that none of these four variables had an additional effect. Normality and homoscedasticity of the residuals of the best-fitting model were visually checked as well as tested via the Shapiro-Wilk test and the Levene's test, and Cook's distance was smaller than 0.5 for all data points.

One individual from introduction C, Mees, was an outlier and was left out of this analysis. Mees was maternally deprived during childhood, showed impaired social behaviour in the social group, and responded to the new male with extreme fear. As the other females in our study grew up in social groups with their mother present, we considered Mees not representative of normal social behaviour during male introductions. The results were in the same direction when taking Mees into account during our analysis, but less significant or non-significant. However, the main outcome and conclusions did not change.

Secondly, we analysed the changes in female behaviour during the increase of female tolerance of the male. To analyse these changes independent of the duration of the introductions, we calculated the rates of female behaviour towards the male for each period of days in which the same number of females had tolerated the male. We refer to these time frames with constant degree of tolerance as 'tolerance bins'. The percentage of females tolerating the male is a measure that is independent of the duration of the introduction. Comparing rates of female behaviour across tolerance bins shows how behaviour changes when additional females tolerate the male. There were 12,8 , and 9 tolerance bins for introductions A, B, and C, respectively. The number of observation days within each bin varied from 1 to 20 days, with an average of $3.38 \pm$ 4.21 days. 
We divided the sum of occurrences of female aggression, unprovoked submission, affiliation, and mating access towards the new male, by the observation time within each tolerance bin (i.e. calculating behaviour rates per hour for each tolerance bin). Subsequently, these rates of female behaviour towards the male were logarithmically transformed using the natural log. General linear models, each with separate intercepts and separate slopes for the three introductions, were fitted to test whether female aggression, submission, and affiliation towards the male, as well as male mating access linearly increased or decreased with integration progress (i.e., the percentage of females tolerating the male). Female behaviour was the dependent variable, while tolerance percentage was entered in each model as predictor variable, and the introduction $(A, B$, or C) was entered as categorical factor. The weight of each data point was set to the number of days a tolerance bin lasted. Normality and homoscedasticity of the residuals of the models were visually checked as well as tested via the Shapiro-Wilk test and the Levene's test, and Cook's distance was smaller than 0.5 for all data points.

All tests were two-tailed with critical significance level set to 0.05 . $R$ version 3.2.3 was used for statistical analyses. For the general linear model we used the packages $/ \mathrm{m}$ (to fit and test general linear models) and anova (to compare different models). Graphics were made with $R$ version 3.2.3.

\section{Ethical approval}

All applicable national, and/or institutional guidelines for the care and use of animals were followed. All procedures performed in studies involving animals were in accordance with the ethical standards of the institution or practice at which the studies were conducted.

\section{Results}

\section{Male behaviour}

All three males were successfully introduced into their respective new group and obtained the alpha position. No clear differences in aggression (average \pm SEM: 11.11 \pm 0.38 ), submission (average \pm SEM: $0.70 \pm 0.70$ ), and affiliation (average \pm SEM: 1.96 \pm 0.69 ) were found between the three new males during the first 5 hours of their introductions (Figure 1a-c). In contrast, a clear difference between the three males was visible in mating access (average \pm SEM: $26.24 \pm 10.11$ ). During the first five hours, the mating rate of the male in introduction $C$ was more than three times higher than the mating rates of the males in introductions $A$ and $B$ (Figure $1 d$ ).

\section{Female tolerance}

A fast increase in the percentage of females tolerating the male during the first 7 days of contact was seen in all three introductions. Thereafter, it took longer before additional females tolerated the new male. At the end of the observation period, $100 \%$ (introduction A), 92\% (introduction B), and 83\% (introduction C) of the females tolerated the male (Figure 2). 

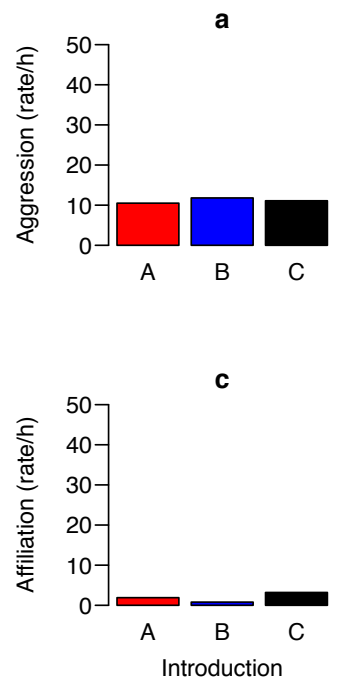

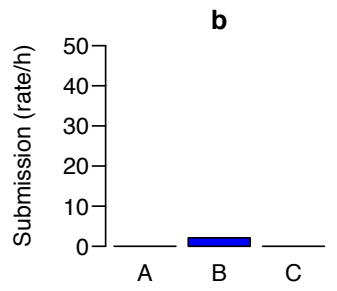

d

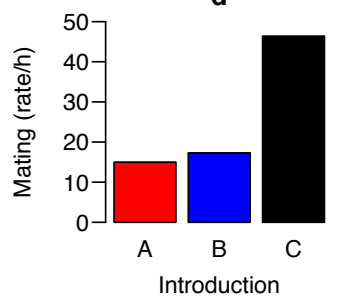

Figure 1 The rates of new male to resident female aggression (a), submission (b), affiliation (c), and mating access (d) within the first 5 hours of the introduction. There are no obvious differences between the males in aggression, submission and affiliation, while the male in introduction $\mathrm{C}$ mated more than twice as much as the other two males.

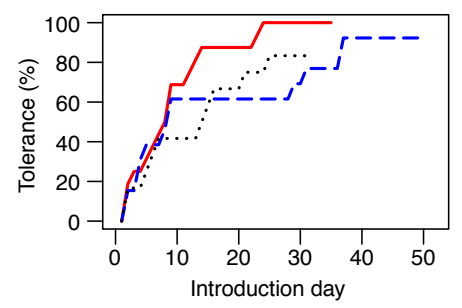

We investigated whether female tolerance of the new male depended on female age, dominance rank, fertility, and the number of female coalitionary partners. Female age ( $\left.G L M, N=40, R^{2}=0.262, p=0.011\right)$, the inverse of female age (1/age) (GLM, $N=40$, $\left.R^{2}=0.545, p<0.001\right)$, and female dominance rank ( $G L M, N=40, R^{2}=0.192, p=0.050$ ) significantly correlated with the timing of female tolerance during the three introductions (Table 1). The number of female coalitionary partners ( $G L M, N=40$, $\left.R^{2}=0.072, p=0.434\right)$ and female fertility ( $\left.G L M, N=27, R^{2}=0.055, p=0.506\right)$ did not significantly influence the timing of female tolerance (Table 1 ). The inverse of age is the best predictor of female tolerance, explaining almost $55 \%$ of the variation in the timing of female tolerance (Table 1 and Figure 3). Adding age, dominance rank, the number of female coalitionary partners, or fertility to this best-fitting model did not add significantly to the model. Each of the extended models was compared with the bestfitted model yielding non-significant F-tests: $F(3,33)=1.07,1.20,1.64$ and $F(2,22)=0.08$, respectively ( $p \geq 0.20$; Table 2 ). We also tested whether the model with separate 
intercepts for each of the introductions added significantly to the best-fitting model (which has the same fitted value for the intercept irrespective of the introduction); this was not the case: $F(2,34)=0.96(p=0.39)$. So, the final, best fitting model has inverse age as the only predictor of the timing of female tolerance and has different slopes for the three introductions, but not different intercepts (Figure 3b). In conclusion, older females tolerated a new male significantly sooner during introductions than did younger females. Other female characteristics did not add significantly to this inverseage effect on the integration progress.

Table 1 The effect of individual female characteristics on the timing of female tolerance during each of the three (or two) introductions, and the overall explained variances $\left(R^{2}\right)$. The second model (Inverse age) is the best-fitting model.

\begin{tabular}{lllll}
$\begin{array}{l}\text { Dependent variable: } \\
\text { Day of tolerance }\end{array}$ & Introduction A & Introduction B & Introduction C & $\begin{array}{l}\text { Variance } \\
\text { explained }\end{array}$ \\
\hline Model & $\mathrm{N}=16$ & $\mathrm{~N}=13$ & $\mathrm{~N}=11$ & $\mathrm{~N}=40$ \\
\hline Age & $\mathrm{t}=-3.425^{* *}$ & $\mathrm{t}=-2.556^{*}$ & $\mathrm{t}=-3.535^{*}$ & $\mathrm{R}^{2}=0.262^{*}$ \\
Inverse age & $\mathrm{t}=3.423^{* *}$ & $\mathrm{t}=6.402^{* * *}$ & $\mathrm{t}=4.483^{* * *}$ & $\mathbf{R}^{2}=\mathbf{0 . 5 4 5 * *}$ \\
Dominance rank & $\mathrm{t}=0.990$ & $\mathrm{t}=3.074^{* *}$ & $\mathrm{t}=2.139^{*}$ & $\mathrm{R}^{2}=0.238^{*}$ \\
Coalition size & $\mathrm{t}=-1.434$ & $\mathrm{t}=-1.070$ & $\mathrm{t}=-0.272$ & $\mathrm{R}^{2}=0.072$ \\
\hline & $\mathrm{N}=16$ & & $\mathrm{~N}=11$ & $\mathrm{~N}=27$ \\
\hline Fertility & $\mathrm{t}=-0.126$ & $\mathrm{x}$ & $\mathrm{t}=1.025$ & $\mathrm{R}^{2}=0.055$ \\
$* 0.05 \geq \mathrm{p}>0.01, * * 0.01 \geq \mathrm{p}>0.001, * * * \mathrm{p} \leq 0.001$ & &
\end{tabular}

Table 2 The additional effect of each of the characteristics Age, Dominance rank, Coalition size and Fertility on the timing of female tolerance during the three (or two) introductions, when adding each of these characteristics to the best-fitting model (Inverse age as only predictor). The last column presents $\mathrm{F}$ values showing that none of these characteristics adds significantly to the best-fitting model.

\begin{tabular}{|c|c|c|c|c|c|}
\hline $\begin{array}{l}\text { Dependent variable: } \\
\text { Day of tolerance }\end{array}$ & $\begin{array}{l}\text { Introduction } \\
\text { A }\end{array}$ & $\begin{array}{l}\text { Introduction } \\
\text { B }\end{array}$ & $\begin{array}{l}\text { Introduction } \\
\mathrm{C}\end{array}$ & $\begin{array}{l}\text { Variance } \\
\text { explained }\end{array}$ & \\
\hline Model & $\mathrm{N}=16$ & $\mathrm{~N}=13$ & $\mathrm{~N}=11$ & $\mathrm{~N}=40$ & F test \\
\hline $\begin{array}{l}\text { Age added } \\
\text { Inverse age } \\
\text { Age }\end{array}$ & $\begin{array}{l}\mathrm{t}=2.515^{*} \\
\mathrm{t}=1.531\end{array}$ & $\begin{array}{l}\mathrm{t}=4.386 * * * \\
\mathrm{t}=0.844\end{array}$ & $\begin{array}{l}t=3.137 * * \\
t=1.166\end{array}$ & $\mathrm{R}^{2}=0.586 * * *$ & $F(3,33)=1.08$ \\
\hline $\begin{array}{l}\text { Dominance rank added } \\
\text { Inverse age } \\
\text { Dominance rank }\end{array}$ & $\begin{array}{l}\mathrm{t}=2.437^{*} \\
\mathrm{t}=0.573\end{array}$ & $\begin{array}{l}\mathrm{t}=4.553 * * * \\
\mathrm{t}=0.636\end{array}$ & $\begin{array}{l}t=2.404^{*} \\
t=1.398\end{array}$ & $\mathrm{R}^{2}=0.576 * * *$ & $F(3,33)=0.80$ \\
\hline \multirow[t]{2}{*}{$\begin{array}{l}\text { Coalition size added } \\
\text { Inverse age } \\
\text { Coalition size } \\
\end{array}$} & $\begin{array}{l}t=2.683 * \\
t=-0.784\end{array}$ & $\begin{array}{l}t=6.510 * * * \\
t=-2.067 *\end{array}$ & $\begin{array}{l}\mathrm{t}=3.413 * * \\
\mathrm{t}=0.274\end{array}$ & $\mathrm{R}^{2}=0.604 * * *$ & $F(3,33)=1.64$ \\
\hline & $N=16$ & & $\mathrm{~N}=11$ & $N=27$ & F test \\
\hline $\begin{array}{l}\text { Fertility added } \\
\text { Inverse age } \\
\text { Fertility }\end{array}$ & $\begin{array}{l}t=2.516^{*} \\
t=0.340\end{array}$ & $\begin{array}{l}x \\
x\end{array}$ & $\begin{array}{l}t=3.294 * * \\
t=-0.172\end{array}$ & $\mathrm{R}^{2}=0.393 *$ & $F(2,22)=0.08$ \\
\hline
\end{tabular}


a

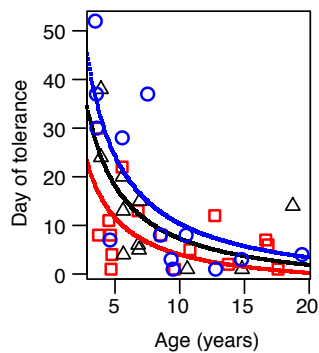

b

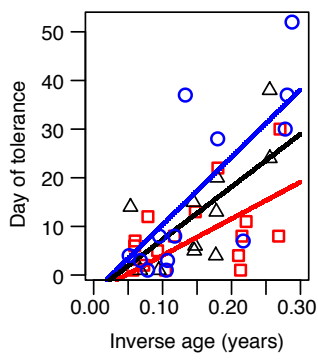

Figure 3 The inverse of female age is the best predictor of the timing of female tolerance during introductions A (red squares), B (blue circles), and $\mathrm{C}$ (black triangles). Older females tolerated the new male sooner than younger females (a). The predicted lines are fitted by GLMs with inverse age as predictor and include different slopes, but not different intercepts (b).

\section{Changes in Behaviour with Integration Progress}

Female aggression, submission, affiliation, and male mating access are expected to change as integration of the new male progresses. For each of these behaviours we fitted a general linear model (GLM) with female tolerance percentage (i.e. the tolerance bins) as a predictor, while including separate intercepts and slopes for the three introductions. Female aggression ( $G L M, N=29, R^{2}=0.486, p<0.001$; Figure 4a) and submission ( $G L M, N=29, R^{2}=0.836, p<0.001$; Figure $4 b$ ) towards the new male decreased significantly with integration progress. Female affiliation towards the male increased significantly as integration progressed ( $G L M, N=29, R^{2}=0.413, p<0.001$; Figure $4 c$ ). Male mating access decreased significantly with integration progress (GLM, $N=29, R^{2}=0.739, p<0.001$; Figure $4 d$ ). For each of the four behaviours, all changes were in the same direction for all three introductions. However, not all slopes differed significantly from zero and effect sizes differed between the introductions (Table 3). In conclusion, a general decrease in female aggression, submission, and male mating access was observed with integration progress, while female affiliation increased.

a

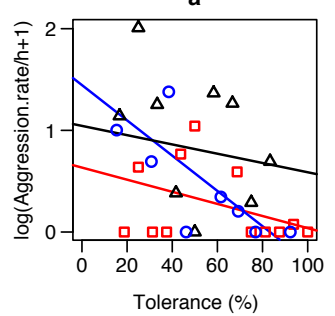

c

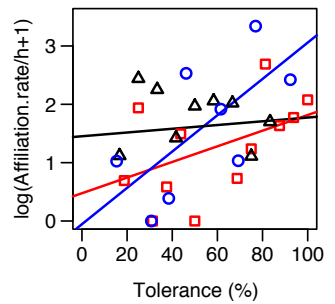

b

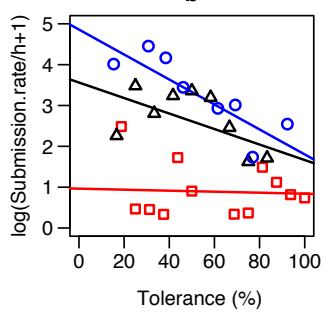

d

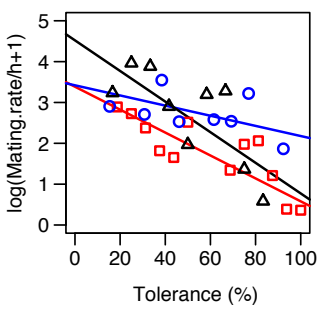

Figure 4 The changes in female aggression (a), submission (b), affiliation (c), and mating access (d) to the new male during introduction $A$ (red squares), B (blue circles), and C (black triangles). The predicted lines are fitted by GLMs that include different slopes and different inter-cepts. 
Table 3 The variance $\left(R^{2}\right)$ in female social behavior towards the new male explained by integration progress (i.e. \% of female tolerance) in the four GLMs. Per introduction the fitted values of the separate intercepts and slopes are given, as well as their statistics.

\begin{tabular}{|c|c|c|c|c|c|}
\hline $\begin{array}{l}\text { Predictor variable: } \\
\% \text { female tolerance }\end{array}$ & $\begin{array}{l}\text { Introduction } \\
\text { A }\end{array}$ & $\begin{array}{l}\text { Introduction } \\
\text { B }\end{array}$ & $\begin{array}{l}\text { Introduction } \\
\text { C }\end{array}$ & $\begin{array}{l}\text { Variance } \\
\text { explained }\end{array}$ & \\
\hline Dependent variable & $\mathrm{N}=12$ & $\mathrm{~N}=8$ & $\mathrm{~N}=9$ & $N=29$ & \\
\hline \multirow{2}{*}{ Aggression } & Intercepts & $\begin{array}{l}0.634 \\
\mathrm{t}=1.702\end{array}$ & $\begin{array}{l}1.445 \\
t=3.716 * *\end{array}$ & $\begin{array}{l}1.044 \\
t=3.130 * *\end{array}$ & \multirow{2}{*}{$\mathrm{R}^{2}=0.486 * * *$} \\
\hline & Slopes & $\begin{array}{l}-0.006 \\
t=-1.233\end{array}$ & $\begin{array}{l}-0.017 \\
\mathrm{t}=-2.708 *\end{array}$ & $\begin{array}{l}-0.005 \\
t=-0.809\end{array}$ & \\
\hline \multirow{2}{*}{ Submission } & Intercepts & $\begin{array}{l}0.946 \\
t=1.930\end{array}$ & $\begin{array}{l}4.859 \\
t=9.314 * * *\end{array}$ & $\begin{array}{l}3.563 \\
\mathrm{t}=7.964 * * *\end{array}$ & \multirow{2}{*}{$\mathrm{R}^{2}=0.836 * * *$} \\
\hline & Slopes & $\begin{array}{l}-0.001 \\
t=-0.192\end{array}$ & $\begin{array}{l}-0.031 \\
t=-3.661 * *\end{array}$ & $\begin{array}{l}-0.019 \\
t=-2.510 *\end{array}$ & \\
\hline \multirow{2}{*}{ Affiliation } & Intercepts & $\begin{array}{l}0.476 \\
t=0.835\end{array}$ & $\begin{array}{l}-0.055 \\
t=-0.083\end{array}$ & $\begin{array}{l}1.453 \\
t=2.848 * *\end{array}$ & \multirow{2}{*}{$\mathrm{R}^{2}=0.413 * * *$} \\
\hline & Slopes & $\begin{array}{l}0.013 \\
t=1.815\end{array}$ & $\begin{array}{l}0.031 \\
\mathrm{t}=3.269 * *\end{array}$ & $\begin{array}{l}0.003 \\
t=0.371\end{array}$ & \\
\hline \multirow{2}{*}{ Mating access } & Intercepts & $\begin{array}{l}3.386 \\
t=5.959 * * *\end{array}$ & $\begin{array}{l}3.421 \\
t=5.766 * * *\end{array}$ & $\begin{array}{l}4.520 \\
t=8.886 * * *\end{array}$ & \multirow{2}{*}{$\mathrm{R}^{2}=0.739 * * *$} \\
\hline & Slopes & $\begin{array}{l}-0.028 \\
\mathrm{t}=-3.810 * * *\end{array}$ & $\begin{array}{l}-0.012 \\
t=-1.308\end{array}$ & $\begin{array}{l}-0.038 \\
\mathrm{t}=-4.357 * * *\end{array}$ & \\
\hline
\end{tabular}

\section{Discussion}

This study identified male and resident female behaviour during male introduction in captive rhesus macaques and showed that female age is the paramount female characteristic that influences a female's attitude towards new males.

\section{Male behaviour}

The initial, relatively high levels of aggression and low levels of affiliation by the three new males is in accordance with previous research (captivity: Rose et al. 1972; Bernstein et al. 1977; wild: Alberts et al. 1992; Zhao 1994; Bercovitch 1997; Teichroeb et al. 2011; Marty et al. 2017). Behavioural differences between the new males may explain variances in female behaviour (van Noordwijk and van Schaik 1985, 2000; Marty et al. 2016). In our study, however, no clear differences were observed between the three new males in initial aggressive, submissive, and affiliative behaviour towards the resident females. Nonetheless, the male in introduction $\mathrm{C}$ had much higher mating access than the other two males. However, we consider male mating access a result of both new male and resident female behaviour, since female cooperation is required. Therefore, our results indicate that there is only minor variation in the males' behaviour. Conversely, we have seen variation in female aggression, submission, and affiliation levels towards the different males. Thus, our data do not show an obvious relation between the male's behaviour at the start of the introduction, and the subsequent integration process. Unfortunately, the current number of introductions was too low to 
produce any meaningful statistics on the possible effect of male behaviour on female behaviour during introductions. Either a male's initial behaviour may not affect how females respond, or the link between male and female behaviour may be less straightforward.

\section{Introduction and integration success}

Linking the integration success (the final female tolerance percentage) to the introduction success (the caretaker's decision to leave the male in the group permanently) can have important implications for introduction management. All three introductions were successful while the final percentages of female tolerance of the male were 83,92 and $100 \%$. This shows that a successful introduction is not tantamount to a $100 \%$ successful integration. Thus, tolerance by a subset of females seems sufficient to decrease the risk of attack by female coalitions drastically (as assessed by the experienced caretakers). Unfortunately, behavioural data on unsuccessful introductions are lacking, making it difficult to identify a possible female tolerance threshold (i.e. minimum percentage of tolerating females) for successful introductions. We aim to fill this knowledge gap in future studies, since approximately 1 in 5 introductions are unsuccessful (unpublished data from the BPRC, collected between 2004 and 2011, $\mathrm{N}=43$ ).

\section{Female characteristics}

Female age, dominance rank, fertility, and the number of female coalitionary partners were expected to affect the timing of female tolerance of the new male. However, only female age determined tolerance: younger females tolerated the male later than older females. The inverse of female age was a better predictor than age itself. Thus, the relationship between female age and tolerance is not linear but negative exponential (Figure 3a). Young individuals were slow in tolerating the new male, but females above a certain age tolerated the male relatively fast. When looking at our data, the breakpoint for quick tolerance may be around the age of 7 years. These females had less experience with male introductions. All of the 7 years and older females had experienced a male introduction before (75\% during adulthood, 25\% as juvenile), while none of the younger females did. This shows that experience with male introductions may be an important factor influencing a female's attitude to an unfamiliar male and could explain the observed effect of the inverse of age. Moreover, younger females may be more fearful during male introductions, since younger individuals generally behave more submissively than older ones (Bernstein and Ehardt 1985). Lastly, the effect of age on female tolerance of the male may overrule the effects of female dominance rank and fertility found in other studies (Kawai 1960; Zaunmair et al. 2015). Dominance rank and fertility possibly come into play after female tolerance, determining male social and mating access at a later stage. 


\section{Changes in female behaviour towards the new male}

Changes in a female's behaviour towards the male during the introduction represent the female's role in the integration process.

\section{Aggression}

Female aggression to the new male indicates the resistance a male encounters during an introduction. As expected, female aggression towards the new male decreased with integration progress, possibly due to increased familiarity. A decrease in aggression with integration progress is in accordance with previous studies on rhesus macaque introductions (Bernstein and Mason 1963; Bernstein et al. 1977). In contrast to our findings, female aggression towards the male was absent when creating a one-male, multi-female lion-tailed macaque (Macaca silenus) group (Zaunmair et al. 2015). Lion-tailed macaques are a relatively tolerant species, while rhesus macaques are considered rather intolerant (Thierry 2000). This indicates there are speciesspecific responses during introductions (Clarke et al. 1995).

We found that aggression during introductions can also differ between introductions within the same species. In two of the three introductions ( $A$ and $B$ ), the rate of aggression was virtually zero after $75 \%$ of the females tolerated the male. In the third introduction (C), however, aggression did not peter out, while at the same time the percentage of females that tolerated the male did not exceed $83 \%$. Although the male in group $\mathrm{C}$ was successfully introduced, there may still have been resistance present among the females against this male, possibly with long-term implications. Indeed, after several months this male had to be removed from the group due to high aggression levels, while the males in the other two introductions stayed in the group for at least three years (BPRC animal register). Thus, very low female aggression levels at the end of the introduction may signal long-term group stability. However, no hard conclusions on possible long-term effects of remaining aggression can be drawn from comparing these three cases only.

\section{Submission}

A decrease in female submission during an introduction may indicate acceptance of the new male's dominant position. As expected, female submission decreased significantly with integration progress. This is in accordance with results from a previous, descriptive, study (Bernstein and Mason 1963). However, when comparing the three introductions, only significant decreases in submission were visible during introductions B and C. During introduction A, female submission remained low and was the lowest of all three introductions (Figure $4 \mathrm{~b}$ ). This variation may be due to differences in female characteristics (e.g. personality) between the three groups or due to male's behaviour. 
Submission levels were low at the end of the three introductions. These low rates of female submission towards the male may signal that the females no longer perceive him as a threat. This is an important step in the tolerance of the new male and thus a crucial aspect of a successful integration. Indeed, introduction A (with the lowest female submission rate) had the fastest female tolerance increase, and the management decision to leave the male in the group full-time was made after only 12 days of contact. Although low submission rates may indicate short-term safety, they appear unrelated to long-term aggression and affiliation patterns (e.g. introduction C). Still, these results suggest that animal caretakers can use a low rate of female submission towards the introduced male as a signal that the male can stay in the group full-time.

\section{Affiliation}

Female affiliation towards the new male increased significantly during the course of the three introductions. It has been suggested that affiliation replaces aggression during introductions (Bernstein et al. 1977). Consistent with this idea, the introduction with the largest decrease in aggression, introduction B, also showed the largest increase in affiliation, and the introduction with the smallest decrease in aggression, introduction $\mathrm{C}$, also showed the smallest increase in affiliation, suggesting that these two social strategies may depend on each other.

An increase in affiliation may indicate that the females started bonding with the male during the introductions. Initial social interactions are important in the formation of social bonds on the longer term (Evers et al. 2015; Dunayer and Berman 2017). The observed affiliation may thus eventually provide the male with a position in the female social network, in which rhesus macaque males usually become well integrated (Hill 1990). Possibly, less or weaker social bonds were formed in introduction C. The longterm effects of the minimal affiliation in this group remains unknown, but the existence of weak social bonds may have led to the removal of the male from the group a few months after the introduction (see above). However, we cannot statistically support this supposition due to our low sample size.

\section{Male mating access}

Male mating access decreased with integration progress, after initial high copulation rates. Female mating interest may decrease with pregnancy; however, female rhesus macaques also mate when pregnant (Loy 1971). Alternatively, the Coolidge effect, i.e. when an individual loses sexual interest in a mating partner that has been available for a longer period of time (Dewsbury 1981), may explain the decrease in mating access. Indeed, primates prefer novel mating partners (Manson 1995; Inoue and Takenaka 2007). The possible existence of a Coolidge effect in rhesus macaques can have important management implications for captive breeding colonies. Mating interest, and therefore reproductive success, may decrease when the male has spent more time in the group. When reproduction rates are lower than desired, likely after a few years, the introduction of a new breeding male may restore mating interest and increase reproduction. 


\section{Conclusion}

To summarize, female aggression and submission towards the new males decreased as integration progressed. Female affiliation increased, and mating rates decreased. In general, the integration progress and the changes in social behaviour were consistent across the introductions, indicating a general pattern in female behaviour during male introductions. Low levels of female submission can play an important role in determining when it is safe to leave a male in the group full-time. Low female-male aggression levels, on the other hand, may indicate long-term group stability. Although a general pattern in female behaviour is discernable, differences in effect sizes and significance within this general pattern cannot be ignored. This may be due to female characteristics, but not to differences in male behaviour. Overall, we conclude that female behaviour can provide valuable information about the integration process during male introductions, that can be helpful in management decisions. 


\section{Appendix 1}

\begin{tabular}{|c|c|c|c|c|}
\hline Name & Gender & Age (years) & Dominance rank & \multirow{6}{*}{$\begin{array}{l}\text { Table A1 The composition of } \\
\text { the Liby group (introduction } \\
\text { A) during the study, and the } \\
\text { characteristics of the animals } \\
\text { in the group. The horizontal } \\
\text { lines separate the different } \\
\text { matrilines in the group. }\end{array}$} \\
\hline Lambik' & Male & 11 & - & \\
\hline $\mathrm{Niby}^{\mathrm{s}}$ & Female & 17 & 1 & \\
\hline $\mathrm{Cloe}^{\mathrm{s}}$ & Female & 10 & 2 & \\
\hline Quran $^{\mathrm{S}}$ & Female & 4 & 3 & \\
\hline Glowy & Female & 0 & - & \\
\hline Ruby $^{\text {s }}$ & Female & 12 & 5 & \multirow{22}{*}{$\begin{array}{l}\text { 'Immigrant male } \\
\text { s Subject of the study }\end{array}$} \\
\hline Zeptos & Female & 4 & 6 & \\
\hline Romano & Male & 0 & - & \\
\hline Femto & Female & 2 & - & \\
\hline Barbie $^{\mathrm{s}}$ & Female & 8 & 4 & \\
\hline Minnie $^{s}$ & Female & 16 & 7 & \\
\hline Escape $^{\mathrm{s}}$ & Female & 9 & 9 & \\
\hline Oka ${ }^{\mathrm{s}}$ & Female & 4 & 10 & \\
\hline Langoer & Female & 0 & - & \\
\hline Karma $^{\mathrm{S}}$ & Female & 3 & 11 & \\
\hline Prison & Male & 2 & - & \\
\hline Shift & Female & 0 & - & \\
\hline Frerin ${ }^{\mathrm{s}}$ & Female & 5 & 8 & \\
\hline Hurley & Male & 0 & - & \\
\hline Maxxie & Female & 2 & - & \\
\hline What $^{\mathrm{s}}$ & Female & 16 & 12 & \\
\hline Millis & Female & 3 & 14 & \\
\hline Then $^{\text {s }}$ & Female & 13 & 13 & \\
\hline $\operatorname{San}^{\mathrm{S}}$ & Female & 6 & 16 & \\
\hline Dra & Female & 2 & - & \\
\hline Bantoe $^{\mathrm{s}}$ & Female & 4 & 15 & \\
\hline B52 & Male & 0 & - & \\
\hline
\end{tabular}




\begin{tabular}{|c|c|c|c|}
\hline Name & Gender & Age (years) & Dominance rank \\
\hline Smack' & Male & 13 & - \\
\hline Marieke $^{\mathrm{s}}$ & Female & 19 & 1 \\
\hline Zeta $^{\mathrm{s}}$ & Female & 14 & 7 \\
\hline Coma $^{\mathrm{s}}$ & Female & 9 & 11 \\
\hline Tehillas & Female & 3 & 12 \\
\hline Suzie $^{\mathrm{S}}$ & Female & 7 & 9 \\
\hline Nasya $^{\mathrm{S}}$ & Female & 3 & 10 \\
\hline $\mathrm{Dai}^{\mathrm{s}}$ & Female & 5 & 8 \\
\hline Han & Male & 3 & - \\
\hline Knoxville & Female & 2 & - \\
\hline Jones $^{S}$ & Female & 12 & 6 \\
\hline Julia & Female & 2 & - \\
\hline Lammie $^{\mathrm{S}}$ & Female & 10 & 2 \\
\hline Pompedomp & Male & 3 & - \\
\hline Tokkie & Female & 2 & - \\
\hline Nora $^{\mathrm{s}}$ & Female & 9 & 13 \\
\hline Mora & Male & 2 & - \\
\hline Raksha $^{\text {s }}$ & Female & 8 & 3 \\
\hline Chills $^{\mathrm{s}}$ & Female & 4 & 5 \\
\hline Thuu $^{\mathrm{s}}$ & Female & 3 & 4 \\
\hline Fifty & Male & 2 & - \\
\hline
\end{tabular}

Table A2 The composition of the Marieke group (introduction B) during the study, and the characteristics of the animals in the group. The horizontal lines separate the different matrilines in the group. Note that one adult female past away in the first week after the introduction and could not be used as a subject in this study.

I Immigrant male

s Subject of the study

\begin{tabular}{|c|c|c|c|}
\hline Name & Gender & Age (years) & Dominance rank \\
\hline Bar' $^{\prime}$ & Male & 9 & - \\
\hline Griotje $^{\mathrm{s}}$ & Female & 6 & 2 \\
\hline Sparrow & Female & 2 & - \\
\hline Chupa $^{\mathrm{s}}$ & Female & 5 & 1 \\
\hline $\mathrm{Clio}^{\mathrm{s}}$ & Female & 18 & 10 \\
\hline Panda $^{\mathrm{S}}$ & Female & 10 & 8 \\
\hline Ling-Lings & Female & 6 & 9 \\
\hline BMW & Male & 1 & - \\
\hline Ping-Ping & Male & 0 & - \\
\hline Zhen-Zhen ${ }^{\text {S }}$ & Female & 3 & 6 \\
\hline Lexus $^{\mathrm{S}}$ & Female & 3 & 12 \\
\hline Infinity & Female & 2 & - \\
\hline Mees $^{s}$ & Female & 17 & 11 \\
\hline Zwaluw ${ }^{\mathrm{s}}$ & Female & 5 & 3 \\
\hline Gier & Female & 2 & - \\
\hline Koolmees & Male & 0 & - \\
\hline River $^{s}$ & Female & 14 & 7 \\
\hline Spekkie ${ }^{\text {s }}$ & Female & 6 & 5 \\
\hline Mrs. Bacon & Female & 0 & - \\
\hline Alblas ${ }^{s}$ & Female & 5 & 4 \\
\hline
\end{tabular}

Table A3 The composition of the Clio group (introduction C) during the study, and the characteristics of the animals in the group. The horizontal lines separate the different matrilines in the group.

I Immigrant male

s Subject of the study 


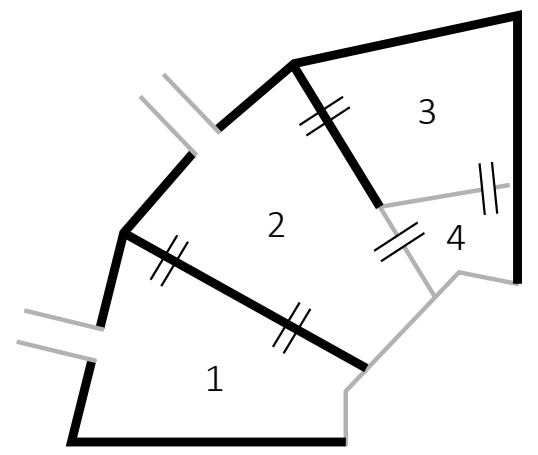

Figure A1 A map of the inside enclosure. The numbers indicate the different compartments. Concrete walls are indicated by the thick black lines. The thick grey lines indicate wire mesh. The thin black lines represent sliding doors that connect the different compartments The location of the tunnels to the outside compartments were connected to either inside compartment 1 and 2 (shown here), or inside compartment 2 and 3. 


\section{Appendix 2}

Table A4 The ethogram used during this study (Altmann 1962; Angst 1974)

BEHAVIOUR DESCRIPTION

\begin{tabular}{ll}
\hline $\begin{array}{l}\text { Aggressive behaviour } \\
\text { immigrant }\end{array}$ & $\begin{array}{l}\text { A female supports another female during an aggressive conflict with the immigrant } \\
\text { male, possibly preceded by check-looks from the supported animal towards the } \\
\text { supporter. The animal supports by displaying aggressive behaviour towards the } \\
\text { immigrant male or his supporters. }\end{array}$ \\
\hline Open mouth & $\begin{array}{l}\text { The animal has a tense body posture, the eyes are wide open and focussed on another } \\
\text { individual. The ears stand out, eyebrows may be somewhat raised, the mouth is open } \\
\text { with lips covering teeth. }\end{array}$ \\
\hline Stare & $\begin{array}{l}\text { The individual has a tense body posture, the eyes are wide open and focussed on } \\
\text { another individual. The ears stand out and the eyebrows may be somewhat raised, the } \\
\text { mouth is closed. }\end{array}$ \\
\hline Lunge & $\begin{array}{l}\text { A sudden movement towards another individual, can include stepping, reaching out with } \\
\text { the arms or stomping with a hand on the ground/an object. There is no physical contact } \\
\text { with the other individual and no chasing. }\end{array}$ \\
\hline Point & $\begin{array}{l}\text { The body is tense and stretched out, the eyes are focussed on another individual. The } \\
\text { body is shaped like an arrow with the head in line with the rest of the body. The } \\
\text { eyebrows are raised, the ears are flat and positioned against the head, the eyes may be } \\
\text { somewhat closed and the lips are positioned in a triangular shape. }\end{array}$ \\
\hline Chase & $\begin{array}{l}\text { An animal aggressively runs after or approaches an individual at higher pace, sometimes } \\
\text { accompanied by other agonistic behaviours. }\end{array}$ \\
\hline Push, pull, slap & An individual is aggressively pushing, pulling or slapping another individual. \\
\hline Bite & An individual aggressively bites another individual.
\end{tabular}

\section{Submissive behaviour}

\begin{tabular}{ll}
\hline Bared teeth & $\begin{array}{l}\text { The eyebrows and forehead are raised, the ears are positioned against the head and the } \\
\text { upper and lower lips are drawn back, displaying teeth and gum. Can be spontaneous (i.e. } \\
\text { unprovoked) or in response to aggressive behaviour of the receiver (i.e. provoked). }\end{array}$ \\
\hline Fear scream & $\begin{array}{l}\text { The individual screams loudly with the eyes and mouth wide open, the eyebrows and } \\
\text { forehead are raised and the ears are positioned against the head. Can be spontaneous } \\
\text { (i.e. unprovoked) or in response to aggressive behaviour of the receiver (i.e. provoked). }\end{array}$ \\
\hline Make room & $\begin{array}{l}\text { An avoiding movement away from another individual when that individual is } \\
\text { approaching. The actor remains in approximately the same spot, and may make room } \\
\text { with moving/repositioning part of its body (less than } 1 \text { meter). Can be spontaneous (i.e. } \\
\text { unprovoked) or in response to aggressive behaviour of the receiver (i.e. provoked, } \\
\text { approach not necessary). }\end{array}$ \\
\hline Give ground & $\begin{array}{l}\text { Walking away from another individual when that individual is non-aggressively } \\
\text { approaching (i.e. coming within one meter of the subject, unprovoked), or after } \\
\text { receiving aggression from that individual (i.e. provoked, approach not necessary). }\end{array}$ \\
\hline Escape/flee & $\begin{array}{l}\text { Moving away from another individual at high pace. Can be spontaneous (i.e. } \\
\text { unprovoked) or in response to aggressive behaviour of the receiver (i.e. provoked). }\end{array}$
\end{tabular}




\begin{tabular}{ll}
\hline Lifting & \multicolumn{1}{c}{ Affiliative behaviour } \\
\hline Lip smacking & $\begin{array}{l}\text { The individual is making smacking movements with the lips and mouth. The movement } \\
\text { individual. Often the ears move towards the head during lifting. } \\
\text { forehead are raised, while the face is directed towards another individual. }\end{array}$ \\
\hline Social grooming & $\begin{array}{l}\text { Taking care of the fur of another individual by pushing aside its fur, pulling hairs and/or } \\
\text { inspecting for foreign objects. Also includes taking care of another animal's teeth or skin. }\end{array}$
\end{tabular}

\section{Sexual behaviour}

\begin{tabular}{ll}
\hline \multirow{2}{*}{ Mount } & $\begin{array}{l}\text { An individual climbs on the hindquarters of another individual, often by clinching its feet } \\
\text { against the thighs of the other. Can be accompanied by less than } 3 \text { pelvic thrusts, } \\
\text { penetration is not necessary. }\end{array}$ \\
\hline \multirow{2}{*}{$\begin{array}{l}\text { Reject } \\
\text { mounting }\end{array}$} & $\begin{array}{l}\text { An individual rejects the mounting attempt of another animal, by moving away (possibly } \\
\text { at high pace) when the mounter is grasping or intents to climb on the hind quarters, or } \\
\text { by sitting down when the mounter attempts to climb on. The actor may show aggression } \\
\text { towards the mounter, or can be accompanied by submissive behaviour. }\end{array}$ \\
\hline \multirow{3}{*}{ Copulation } & $\begin{array}{l}\text { A sexually reproductive male climbs on the hindquarters of a female, often by clinching } \\
\text { his feet against the thighs of the female. The male shows continuous pelvic thrusts, al } \\
\text { least 3, and penetration is necessary. }\end{array}$ \\
\hline \multirow{2}{*}{ Forced mating } & $\begin{array}{l}\text { The male prevents a female from rejecting his mating attempt by using force or threat, } \\
\text { or succeeds to mate with her within } 5 \text { seconds after a rejection. This includes forcing her } \\
\text { to stand up after sitting down and preventing her from sitting down and/or moving away. }\end{array}$
\end{tabular}

\section{Proximity behaviour}

\begin{tabular}{ll}
\hline Being together & An individual is within 1 meter of another individual, for at least 3 seconds. \\
\hline Approach & $\begin{array}{l}\text { An individual moves into the proximity, within } 1 \text { meter, of another individual and } \\
\text { becomes stationary at this position for at least } 3 \text { seconds. However, this will not be the } \\
\text { case when an approach is prevented. }\end{array}$ \\
\hline $\begin{array}{l}\text { Accepting } \\
\text { approach }\end{array}$ & $\begin{array}{l}\text { The approacher is accepted for more than } 3 \text { seconds within } 1 \text { meter of the approached } \\
\text { animal. }\end{array}$ \\
\hline Reject & $\begin{array}{l}\text { The approached individual moves within } 3 \text { seconds from less than } 1 \text { meter to more than } \\
\text { approach }\end{array}$ \\
$\begin{array}{l}1 \text { meter from the approacher. The approacher does not move away. Only score when } \\
\text { the approacher settles itself in the proximity of the rejecter (when rejected after the } \\
\text { approacher settles), or where the rejecter was stationary before rejecting. }\end{array}$ \\
$\begin{array}{l}\text { Prevent } \\
\text { approach }\end{array}$ & $\begin{array}{l}\text { The approacher is prevented from joining the approached individual and is not accepted } \\
\text { within } 1 \text { meter for more than } 3 \text { seconds. The approacher moves away, often after } \\
\text { receiving aggression from the approached individual. The approached individual does } \\
\text { not move away. }\end{array}$
\end{tabular}




$$
\begin{aligned}
& 30 \\
& 4
\end{aligned}
$$





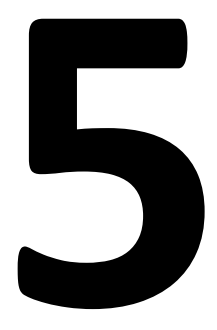

\section{Factors determining male introduction success and long-term stability in captive rhesus macaques}

Astrid Rox, André H van Vliet, Jan AM Langermans,

Elisabeth HM Sterck, Annet L Louwerse 


\begin{abstract}
The entrance of new males into non-human primate groups bears high social risk, yet migration is necessary to prevent inbreeding. Males are not always accepted in their new group. In the wild, males may increase the likelihood of successful group entry by choosing a new group based on their own and the group's characteristics. Understanding whether these characteristics also determine a male's ability to enter captive groups is crucial to improve introduction management. This study aims to identify which factors determine male introduction success (i.e. male stays in the group for at least 4 weeks) and long-term stability (i.e. the male does not cause considerable behavioural problems after success) after male introductions in captive groups of rhesus macaques (Macaca mulatta), creating one-male groups. We studied 64 male introductions at the breeding colony of the Biomedical Primate Research Centre in Rijswijk, The Netherlands. 49 (77\%) introductions were successful, with the male obtaining a long-term stable social position in the group in 38 (59\%) introductions. Introductions of males that reached at least prime age, into groups with more adult females, but without pregnant females were most successful. Moreover, long-term stability was highest when males were heavier, were at least 3.5 years old when they were first removed from their natal group, and groups had few matrilines and no pregnant females were present. Males should be introduced at the time they would naturally immigrate, when they are strongest. Moreover, groups should consist of few large matrilines, as observed in the wild, with philoatric females and males that are removed at natural age. Our study highlights the importance of composing naturalistic groups and mimicking natural migration patterns to maintain long-term stable breeding groups in captivity.
\end{abstract}

\title{
Introduction
}

Primates are highly social animals with the behavioural need to display a wide variety of complex social behaviours. The welfare of captive primates can be enhanced through naturalistic group housing, which will enable them to display natural social behaviour (Mallapur and Choudhury 2003; Lutz and Novak 2005). Naturalistic group housing is common in zoos, while primates in research facilities are generally housed in unnatural groups. Especially pair-housing is common in research facilities. It is therefore not surprising that much research has been done on the management of pair-housed individuals. It is known which animals should be introduced to each other during pair-formation, and which introduction technique is best (Line et al. 1990; Crockett et al. 1994; Reinhardt et al. 1995; Doyle et al. 2010; Truelove et al. 2017; Worlein et al. 2017). However, introducing animals into larger and more naturalistic groups is considered more difficult and more risky than pair-formation, as primate groups have complex social dynamics. Yet, there is very limited knowledge on the management of naturalistic primate groups, while introducing new males is necessary to prevent inbreeding. The Biomedical Primate Research Centre (BPRC) in Rijswijk, the Netherlands is a research facility that houses macaques (Macaca spp.) in naturalistic one-male groups. Their primates live in groups wherein natural group composition and migration patterns are mimicked to optimize primate welfare. 
Wild macaques live in multi-male multi-female groups with male biased migration to prevent inbreeding (Makwana 1978; Marty et al. 2017). Groups generally consist of a few matrilines with multiple adult females, their non-adult offspring and several adult males. Females are phylopatric and remain in their natal group during their lives (de Ruiter and Geffen 1998; Lutz and Novak 2005; Oates-O'Brien et al. 2010). Males migrate from their natal group when reaching sexual maturity, and may change groups several times in their lives (Dittus 1975; van Noordwijk and van Schaik 1985; Zhao 1994; Lutz and Novak 2005; Georgiev et al. 2016; Marty et al. 2017). The arrival of a new male into an existing group leads to extensive male-male competition, commonly resulting in severe injury or sometimes even death (Dittus 1975; Zhao 1994; Lutz and Novak 2005; Georgiev et al. 2016), or unsuccessful immigration (Packer and Pusey 1979; van Noordwijk and van Schaik 1985). This male-male competition is often prevented in naturalistic captive groups through the formation of single-male multifemale (i.e. harem) groups. The resident breeding male is generally removed from these groups before a new male is introduced. Still, similar to male immigration in the wild, male introductions into captive multi-female groups are associated with high aggression levels and stress (Rose et al. 1972; Bernstein et al. 1977; Rox et al. 2018). Moreover, males may fail to enter a group, leading to unsuccessful introductions ((Bernstein et al. 1977), records BPRC). Furthermore, even after successful group entry a wild macaque male may not be able to obtain a long-term stable position in his new group (van Noordwijk and van Schaik 1985, 2000). Similarly, successful introductions may lead to socially instable groups in captivity (Bernstein et al. 1977; Truelove et al. 2017; Rox et al. 2018) where some individuals need to be separated. Thus, not only a male's ability to enter a group initially is crucial for introduction management, also a male's ability to obtain a stable social position in the group is highly important. Composing long-term stable groups should be a main goal of introductions, as animals in socially instable situations often suffer from stress and injuries through increased aggression levels (Sapolsky 1983, 1992; Shively et al. 1986; Crockford et al. 2008). Moreover, the frequency of risky introductions can be reduced when males maintain their position in a group for a longer period of time. In the wild, migrating males may increase the likelihood of successful group entry and obtaining a stable social position by timing their migration with the least female resistance, waiting for optimal body condition (Marty et al. 2017), and by preferably entering groups with the least resistance to new males. Applying knowledge obtained from the wild may increase introduction success and long-term stability.

First, the timing of male group entry may play a role. Male migration in seasonal breeding species (e.g. wild rhesus macaques, Macaca mulatta) is concentrated right before or early in the breeding season (Lindburg 1969; Boelkins and Wilson 1972; Drickamer and Vessey 1973; Cheney and Seyfarth 1983; Mehlman 1986; Sprague 1992; Sussman 1992; Hayakawa and Soltis 2011), as females may be more receptive to new males during this period. Captive male rhesus macaque introductions are indeed more successful during the breeding season, compared to outside the breeding season 
(Bernstein et al. 1977). This implies that timing male introductions with the time frame of natural immigration may increase the chances of male group entry in captivity.

Second, male characteristics may affect a male's ability to successfully enter a group. Single-male introductions into multi-female groups mimic the so-called bluff strategy observed in the wild (van Noordwijk and van Schaik 1985; Sprague 1992; Suzuki et al. 1998; Hayakawa and Soltis 2011; Georgiev et al. 2016; Marty et al. 2016). Bluff immigrants attempt to obtain the alpha position directly after entering a new group. Sub-adult, juvenile, and old males are less successful as bluff immigrants than full-grown prime adult males (Boelkins and Wilson 1972; Sprague 1992; Suzuki et al. 1998; Jack and Fedigan 2004). In addition, males may not enter a new group before they reach high body mass. These heavier males are more successful in taking over a new group (Packer 1979; Marty et al. 2017). Taken together, this suggests that males at their prime age and males with higher body mass may be more successful during introductions than younger, older, or lighter males. However, not only a male's strength may determine his ability to enter a group and maintain a social position, they will also need social skills. Males may gain social skills in their natal group. On average, male macaques leave their natal group at the age of almost 4, with variation from 3 to 8 years (Boelkins and Wilson 1972; Drickamer and Vessey 1973; Mehlman 1986) and $90 \%$ of the males have left their natal group at an age of 6 years (Drickamer and Vessey 1973). Removing animals too early from their social group in captivity may lead to inadequate social behaviour (Bastian et al. 2003; Kempes et al. 2008; Veenema 2009), thereby negatively affecting a male's ability to settle in a new group. Additionally, males may gain social experience in non-natal breeding groups, since they may change groups several times in their lives (Sprague et al. 1998; Kuester and Paul 1999; van Noordwijk and van Schaik 2000). Males may use their social experience gained in a previous group to increase chances of successful group entry during their second immigration. Therefore, captive males with experience as breeding male may be more successful and more long-term stable during introductions.

Third, the composition of the group may affect a male's ability to enter a new group. Males likely enter groups with a composition that increases the chances of successful entry. Males may prefer new groups with a large number of females, as sexual attraction to females is the likely reason for males to migrate (Dittus 1975; Cheney and Seyfarth 1983). Yet female resistance may be larger in groups with more females, as female coalitions against new males may be larger (Packer and Pusey 1979; Cooper et al. 2001; Rox et al. 2018). Possibly, the males balance their preference for more females with the additional costs of increased female resistance. In line with this balance, the number of females does often not affect a male's group choice (Boelkins and Wilson 1972; Drickamer and Vessey 1973; Henzi and Lucas 1980; Cheney and Seyfarth 1983; van Noordwijk and van Schaik 1985; Marty et al. 2016). In addition, females often form coalitions with their family members (Widdig et al. 2006), and the presence of family is important to maintain group stability (Ehardt and Bernstein 1986; Thierry 1990; OatesO'Brien et al. 2010). Groups with a large number of families (i.e. matrilines) may be 
more unstable, which is increased even further through introductions (Ehardt and Bernstein 1986; Oates-O’Brien et al. 2010; McCowan et al. 2018). This indicates that introductions into groups with few females and few matrilines may be more successful. Female reproductive state may affect their attitude towards new males, and their tendency to join coalitions. Lactating and pregnant females can be at risk of infanticide when a new male enters their group (Beehner et al. 2005; Engh et al. 2006; Zaunmair et al. 2015), and may show more resistance to a new male (Pereira and Weiss 1991; Ichino 2005). Moreover, the presence of sub-adult males in a group may affect male group entry. Male long-tailed macaques (Macaca fascicularis) avoid immigration into groups with many sub-adult males (van Noordwijk and van Schaik 2000), while immigrant crested macaques ( $M$. nigra) often enter groups with more males (Marty et al. 2016). In Japanese macaques ( $M$. fuscata), young males preferred to enter groups with many other males, while older males preferred groups with few adult males (Suzuki et al. 1998). Overall, the number of females and matrilines in a group, female reproductive state, and the presence of sub-adult males may affect a male's group choice in the wild. Determining whether or how these factors also affect male introduction success and long-term stability is crucial to improve captive introduction management.

This study aims to identify the factors affecting male introduction success and longterm stability in captive rhesus macaques. We studied the effect of introduction timing, male characteristics and group characteristics during single-male introductions (i.e creating one-male groups) in the rhesus macaque breeding colony of the Biomedical Primate Research Centre in Rijswijk, the Netherlands. Firstly, we expect introductions right before or early in the breeding season to be more successful than introductions later in or outside the breeding season. Secondly, we expect prime aged males, males with higher body mass, more experienced males, and males who left their natal group at a natural age to be more successful during introductions. Lastly, introductions into groups with fewer females, fewer matrilines, without sub-adult males, without pregnant females, and with fewer lactating females may be more successful. We expect similar patterns for long-term stability.

\section{Methods}

\section{Subjects}

Data were collected on 64 male rhesus macaque introductions into existing breeding groups at the Biomedical Primate Research Centre (BPRC) in Rijswijk, The Netherlands. We defined existing breeding groups as groups with multiple adult females (age $\geq 3$ ) and their non-adult offspring, who had already lived together with a breeding male prior to the studied introduction. Natural migration patterns are mimicked in these groups, as females remain in their natal group during their lives and males are generally removed when reaching sexual maturity. These removed natal males are housed in small (i.e. 2-8 individuals) all-male groups for several years, before they may be 
introduced as a breeding male. This form of naturalistic group-housing increases animal welfare and provides better animals for biomedical research, as it increases repeatability and reproducibility of the results (Hannibal et al. 2017), and thereby contributes to refinement and reduction.

The studied groups ranged in size from 4 to 32 animals (average: 15.8), with 2 to 16 adult females (average: 7.4 ) divided over 1 to 7 different matrilines (average: 3.0 ) in each group. $30 \%$ of the females (range: $0-80 \%$ per group) were lactating during the introductions (i.e. they had an infant aged $<1$ year at the start of the introduction). Pregnant females were present in 19 groups, varying from 1 to 6 pregnant females (average: 2.1) per group. Females were considered pregnant if they gave birth to an infant fathered by a male (i.e. previous breeding male or natal male) that was removed from the group before the introduction started. When a natal male that remained in the group during the introduction fathered a child, females were considered pregnant if they gave birth less than 4.5 months after the introduction started. Paternity was determined based on genetics (Sterck et al. 2017). These natal males (age $\geq 3$ ) were present in 20 of the groups; with on average 1.75 (range: 1-4) natal males in these groups.

During the 64 introductions, 49 different males were introduced; 34 males were studied once, while 15 males were studied during introductions into two different groups. As only few males were used multiple times, male identity could not be included as a factor in our analysis. However, 3 out of 4 studied male characteristics change over time. Therefore, we considered multiple introductions of the same male as independent data points. The same is true for the groups the males were introduced to. Generally, introductions were separated in time by several years. Group composition, and thereby dynamics, changes over time. Individuals may be born into the group, may die or mature, or can be removed. Sometimes, groups are even split or female rank reversals take place. Groups sampled at different time points are therefore not comparable. Only once, a group experienced a male introduction twice in one year. However, several adult females causing trouble when introducing new males were removed from the group between the two introductions, changing the group composition and dynamics.

Experienced males were introduced in $42 \%(\mathrm{~N}=27)$ of the cases. Males were considered experienced if they spent time as a breeding male in a social group prior to the studied introduction. The males' ages varied from 4.25 to 22.16 years (average: 9.63), while the ages at which they were removed from their natal group varied from 0.38 to 9.17 years (average: 3.89 ). All animals born at the BPRC since 2001 are groupreared and left their natal group when they were at least 2.5 years old. All males had the opportunity to mature after being removed from their natal group. No males were directly transferred from their natal group to a new breeding group. All males were unfamiliar and unrelated to the groups they were introduced into.

The groups were housed in spacious inside and outside enclosures of approximately $3 \mathrm{~m}$ high and measuring approximately $280 \mathrm{~m}^{2}$ in total. The enclosures contained multiple environmental enrichment items, such as climbing structures, fires hoses, 
tires, and a swimming pool (Vernes and Louwerse 2010). The enclosures could be separated into different compartments. During introductions, the group's access to some of these compartments could be restricted. For a detailed description of the housing conditions during the introductions see Rox et al. 2018. The animals always had plenty of opportunity to avoid each other and they could make use of visual barriers.

\section{Introductory procedure}

The studied introductions were management procedures carried out by experienced animal caretakers, between 2003 and 2016. 25 of the introductions started right before or early in the rhesus macaque breeding season (i.e. between September and December), while 39 introductions were started at the end of or outside the breeding season. The rhesus macaque breeding season at the BPRC lasts from October until March. All introductions followed the BPRC introduction guidelines, wherein males are familiarized with and introduced to the group stepwise. The experienced animal caretakers closely monitored the introductions. During the first steps, a male only spent time with the group under supervision. When the introductions progressed, the animal caretakers' supervision gradually decreased up to the point no supervision was present. The animal caretaker decided whether supervision was needed and when the introduction could progress, based on personal knowledge and experience. The risk of severe aggression and the interest between the group and the new male were estimated.

Prior to an introduction, the previous breeding male was removed from the group, often together with all three year and older natal males. Generally, this took place approximately one year before the introduction. How long a group spent without a male depended on the presence of infants, the number of animals born in the breeding colony, and the genetic representation of the group in the breeding colony. Then, a familiarization phase started several weeks before an introduction. First, the male was moved into an inside compartment that separated him from the group through a concrete wall. This situation allowed auditory, olfactory, and minimal visual contact between the group and the new male. Next, the male was provided with full-visual, and limited physical contact with the group through wire mesh. This could last from a few hours up to a week. The next step depended on the behaviour of the animals. If aggression levels remained high for a longer period of time, the animal caretaker chose to familiarize a single female or a small group of females with the male. The male and the female(s) would spend a few hours up to two days in the same compartment of the enclose. Then, either a new (group of) female(s) was familiarized with the male, or the physical introduction started. If female aggression levels through the fence diminished quickly and the group was interested in the new male, the physical introduction was started immediately. During two of the introductions in our sample, aggression remained high during the familiarization period. These introductions were stopped and were classified as unsuccessful. 
The first step of the physical introduction was introducing the male to the entire group in the outside enclosure. Generally, the male spent 1 to 1.5 hours with the females on the first day. This time was gradually increased as the introduction progressed. Next, additional access to the inside enclosure was provided when the male spent time with the group. Eventually, the male spent approximately 7 hours per day with the females. When the group appeared stable, the male remained in the group full-time. This occurred on average after 44 days (median: 12 days). However, the duration of the introductions varied from 1 to 357 days, with only six introductions exceeding a 100-day period. The exact timing could differ between the introductions, based on the animals' behaviour. Some aggression during introductions is normal, and aggression levels are often elevated at the start of male introductions (Rox et al. 2018). In general, conflicts between animals are brief, and are necessary to confirm their position towards unfamiliar individuals. The animal caretakers only interfered in aggression and stopped the introduction if severe aggression remained between the group and the new male, or the male or females were (at risk of being) severely injured during the introduction. Aggression could generally be stopped from outside the enclosure by either producing loud noise, opening and closing hatches in the enclosure, or pretending to open the door to the enclosure to enter. Then, males could be removed from the group, by moving them into a compartment wherein a concrete wall separates them from the group, Often, the males are happy to move away from the group after interfered conflicts. Therefore, males generally move to the separated compartment voluntarily. In two cases the introduction appeared to progress well for a few weeks, but the male was found dead due to female inflicted injuries.

\section{Data collection}

Data were collected from the digital database of the BPRC. This database contains the main characteristics of each animal at the BPRC (e.g. gender, date of birth, etc.), and includes reports on management procedures (e.g. moving to new enclosure), childbirths, and genetic information (e.g. relatedness to other individuals). Moreover, the database contains information on all introductions, in the form of the summarized administration of the animal caretakers. The start date of each of the different familiarization and introduction steps, the end date of the introductions, and important behavioural events (e.g. female interest and severe aggression) were noted. We retrieved this information on all male introductions and determined the composition of the group a male was introduced to. We selected all male introductions into existing breeding groups at the current housing facilities of the BPRC breeding colony for our analysis.

The raw data is available at: https://doi.org/10.1371/journal.pone.0219972.s001

\section{Ethics statement}

The introductions concerned management procedures necessary to prevent inbreeding at the BPRC breeding colony and were not experimentally induced. This study is a retrospective analysis of these introductions and only used information from 
introduction reports. The introductions adhered to all institutional, national and European animal welfare standards. No invasive research or experimental procedures requiring ethics approval according to the European Directive 2010/63 and the Dutch law were performed. Therefore, no approval by the BPRC animal ethics committee was required. Nevertheless, the management procedures were communicated with and approved by the institute's Animal Welfare Body (IVD BPRC). Introductions were performed under supervision of an experienced ethologist and all animals were under close observation by one of the veterinarians of the BPRC.

\section{Measures}

All measurements were calculated based on the start date of an introduction, defined as the first day a male was physically introduced into the entire group. The majority of variables included in our analysis were transformed into categorical variables, because either the expectations were non-linear or the variables did not meet the assumptions for our statistical models (see below: Statistics). Whether the introductions took place right before or early in the breeding season or not (early versus late), and whether the introduced males were experienced (experienced versus inexperienced) were already categorical factors. Male age at the start of the introduction was divided into three categories. Males between the age of 7 and 12 years were classified as prime aged males, based on data from free-ranging rhesus macaques (Bercovitch 1997) and the notion that animals in captivity generally mature faster than in the wild (Regan and Kitchener 2005). Males before their prime age where classified as young, while males after prime age were defined as old. A similar classification was made for the age the animals were removed from their natal group. Males removed from their natal group between the age of 3.5 and 6.5 years would have left their natal group at normal age (Drickamer and Vessey 1973; Mehlman 1986; van Noordwijk and van Schaik 2000). Males that were removed from their natal group before the age of 3.5 were classified as early, while males removed after the age of 5.5 were classified as late migrants. For one male, information on the age he was removed from this natal group was lacking. This missing data point was filled with the average age (3.9 years) at which the males in our sample were removed from their natal group. Of the 26 introductions with early males, 22 were with males that were raised in peergroups. These peer-reared males were generally introduced to a group during the transition period from the old single-mating system (i.e. individually housed females with infants being transferred to peer-groups after infancy) to the current naturalistic breeding groups at the BPRC between 1996 and 2001 (Kempes et al. 2008), or concerned males from other research institutes with 'standard' breeding conditions (i.e., peer-groups formed following infancy) introduced into BPRC groups to increase genetic variability in the colony. Next, the number of matrilines, i.e. a group of animals that descended from the same female ancestor, was divided into two categories; groups with 3 or fewer matrilines are considered to have few matrilines (i.e. similar to the natural number in macaques (de Ruiter and Geffen 1998; Larson et al. 2018)), while the remaining groups had many matrilines. Moreover, we indicated whether 
there was at least one natal male aged three or more present in the studied group. Similarly, we used the presence or absence of pregnant females in our analysis. The number of females in a group, the percentage of lactating females in the group, and male body weight were the only continuous variables in our models. However, the number of females was logarithmically transformed to fit the assumptions of the model. Generally, males were weighted when they moved to the enclosure adjacent to the females. If this was not the case, we used the last known body weight of the male before the introduction. On average, the males were weighed 96 days (range: 0-359) before their introduction started. An overview of all different predictor variables, their categories and definitions, and their descriptives can be found in table 1.

Table 1 The predictor variables used in this study, including the different categories, their definition, and their descriptives ( $N$, average and SD).

\begin{tabular}{|c|c|c|c|c|}
\hline Variable & Categories & Definition & Sample size & Average ( $\pm S D$ ) \\
\hline \multirow[t]{2}{*}{ Timing } & Early & Sept. - Dec. & $\mathrm{N}=25$ & n.a. ${ }^{1}$ \\
\hline & Late & Jan. - Aug. & $N=39$ & n.a. ${ }^{1}$ \\
\hline \multirow[t]{3}{*}{ Male age } & Young & $\leq 6$ years & $N=22$ & $5.8( \pm 0.7)$ \\
\hline & Prime & $7-12$ years & $N=28$ & $9.5( \pm 2.0)$ \\
\hline & Old & $\geq 13$ years & $\mathrm{N}=14$ & $16.0( \pm 2.7)$ \\
\hline Body weight & Cont. $^{2}$ & Body weight in kg & $N=64$ & $11.1( \pm 2.0)$ \\
\hline \multirow[t]{2}{*}{ Experience } & Yes & Was already breeding male & $N=27$ & n.a. ${ }^{1}$ \\
\hline & No & New breeding male & $N=37$ & n.a. ${ }^{1}$ \\
\hline \multirow[t]{3}{*}{ Natal age } & Early & $<3.5$ years & $N=26$ & $1.6( \pm 1.2)$ \\
\hline & Normal & $3.5-5.5$ years & $N=24$ & $4.8( \pm 0.6)$ \\
\hline & Late & $>5.5$ years & $\mathrm{N}=14$ & $6.3( \pm 0.9)$ \\
\hline Females & Cont. $^{2}$ & Log10(number of females) & $\mathrm{N}=64$ & $7.4( \pm 2.9)$ \\
\hline \multirow[t]{2}{*}{ Matrilines } & Few & $\leq 3$ matrilines & $\mathrm{N}=41$ & $2.2( \pm 0.8)$ \\
\hline & Many & $\geq 4$ matrilines & $N=23$ & $4.3( \pm 0.8)$ \\
\hline \multirow[t]{2}{*}{ Pregnant females } & Yes & $\geq 1$ pregnant females & $N=19$ & $2.1( \pm 1.2)$ \\
\hline & No & 0 pregnant females & $N=45$ & 0 \\
\hline Lactation & Cont. $^{2}$ & $\%$ females with infant $<1$ year & $\mathrm{N}=64$ & $30.5( \pm 24.5)$ \\
\hline \multirow[t]{2}{*}{ Natal males } & Yes & ${ }^{3} 1$ natal males aged 3 or more & $\mathrm{N}=20$ & $1.8( \pm 1.0)$ \\
\hline & No & 0 natal males aged 3 or more & $N=44$ & 0 \\
\hline
\end{tabular}

${ }^{1}$ Non-numerical variable, ${ }^{2}$ Continuous variable

All these variables were used to predict introduction success and whether a male obtained a long-term stable position in the group, called long-term stability in the remainder of this paper. An introduction was successful when the male remained in the group full-time for at least 4 weeks (Rox et al. 2018). Whether the male obtained a long-term stable position was determined based on the removal of the male from the 
group. If the male was long-resident and was removed from the group for management reasons (e.g. preventing inbreeding with adult daughters), the introduction was longterm stable. When the male was removed from the group due to a behavioural problem (e.g. severe aggression or wounding by or to females) the group was not long-term stable. Two males were still in their new group at time of writing, and already spent 2.7 and 2.8 years in their groups. Up to now, they did not cause or experience any considerable behavioural problems and are not expected to do so in the future. Therefore, we treat the introductions of these males as long-term stable.

\section{Statistics}

We used stepwise backwards logistic regression models to identify the effect of the above-named measures on introduction success and on long-term stability. Three separate models were run to test for the effect of introduction timing, male characteristics, and group characteristics on either introduction success or long-term stability. Introduction success and long-term stability were entered as the dependent variables, each in three models, while the predictor variables varied (Table 1). In the first model, the predictor variable was whether the introduction took place in the breeding season (i.e. timing). In the second model, male age, body weight, experience, and the age a male was removed from his natal group (i.e. male characteristics) were entered as predictor variables. The third model contained the number of matrilines, the number of females, the presence of pregnant females, the presence of natal males ages three or more, and the percentage of females with an infant (i.e. group characteristics) as predictor variables. When combining any of two categorical predictor variables included in the same model (e.g. determining how many groups with pregnant females also contained natal males aged three or more), all combinations of the variables occur at least once. One combination occurred once, two combinations occurred three times, while all other combinations occurred at least 5 times. However, there is one exception; there are no experienced young males in our sample. Therefore, we ran an additional model on introductions of prime and old males only.

Model selection was based on the Akaike information criterion (AICc). In each step, the variable that resulted in the lowest AICC after removal was removed from the model, until removing additional variables did not lower AICc further. The remaining model was considered the best predicting model. However, not every variable in the model may be of equal importance (i.e. contribute to the best fit). We considered a variable an important predictor of introduction success or long-term stability if delta AICc was close to or larger than 2 when removing the factor from the best predicting model. When delta AICc is much lower than 2, the factor is not considered an important predictor of introduction success or long-term stability. After selecting the best predicting models, we calculated Chi-square and McFadden's pseudo $R^{2}$ (McFadden 1973) to estimate the variance explained by the model. Finally, post-hoc testing was performed to identify the direction of the effect, or which categories within the predictor variables differed from each other. This was done by including the single predictors in the linear regression models. 
Analysis were done using R studio version 1.1.4, with significance level set to $p \leq 0.05$. Collinearity within the models was checked; the variance inflation factor (VIF) did not exceed 2.95. Moreover, we tested the interdependence of the predictor variables within the same model (see Results section). Figures were created using the Effects package and visualize the outcome of the best predicting model.

\section{Results}

\section{Interdependence of the predictor variables}

The interdependence between predictor variables within the same model were tested. When related factors are included in the same best predicting model, we will provide additional analysis to identify which factor if the main predictor. Male age was related to male body weight ( $A N O V A, F(2,61)=4.209, p=0.019$ ), male experience (Chisquare test: $\left.\chi^{2}=26.221, p<0.001\right)$ and the age at which a male was removed from his natal group (Chi-square test: $\left.\chi^{2}=12.716, p<0.013\right)$. In fact, young males were less heavy than prime and old males (Tukey HSD, $p=0.014$ ), and were never experienced. Moreover, early removal from the natal group was less often present in young males (14\%) than in prime (46\%) and old $(77 \%)$ males. There was no difference in body weight between the experienced and inexperienced males ( $t$-test: $t=0.857, p=0.395$ ), or between males that were removed from their natal group at different ages (ANOVA: $F(2,61)=0.074, p=0.929)$. Finally, male experience was unrelated to the male's rearing condition (Chi-square test: $\chi^{2}=4.472, p=0.106$ ). Thus, male age was related to all other factors in our model, while all other factors were unrelated. Therefore, we ran our analysis on male characteristics twice, once with the entire sample and once with the young males excluded.

Next, the interdependence between the predictor variables in the model concerning group characteristics was tested. The number of females in the group was not related to the presence of pregnant females ( $t$-test: $t=0.401, p=0.690$ ), the number of infants in a group (Pearson correlation test: $\mathrm{R}=0.022, \mathrm{p}=0.865$ ), and the number of matrilines in the group (t-test: $t=1.730, p=0.090)$. In contrast, groups containing natal males aged three or more also contained more females ( $t$-test: $t=2.571, p=0.013$ ). The presence of natal males aged three or more was not related to the presence of pregnant females (Chi-square test: $\chi^{2}=0, p=1.000$ ), the presence of infants in the group ( $t$-test: $t=0.341$, $p=0.735)$, or the number of matrilines in a group (Chi-square test: $\chi^{2}=0, p=1.000$ ). Moreover, the presence of pregnant females was unrelated to the presence of infants (t-test: $t=0.621, p=0.539$ ), and the number of matrilines in the group (Chi-square test: $\chi^{2}=0, p=1.000$ ). Finally, there neither was a relation between the number of matrilines and the presence of infants ( $t$-test: $t=0.160, p=0.874$ ). Thus, the group characteristics are independent of each other, except for the presence of natal males aged three or more and the number of females in a group. 


\section{Introduction success and long-term stability}

A new male was successfully introduced to a group during $77 \%(N=49)$ of the 64 introductions. 77\% ( $\mathrm{N}=38)$ of the successfully introduced males did not cause considerable behavioural problems in the group in the years after and were eventually removed from the group for management reasons (i.e. were long-term stable). Altogether, $59 \%(\mathrm{~N}=38)$ of all introduced males were able to obtain a long-term stable position in their group (table 2).

Table 2 The number and percentage of successful and unsuccessful introductions, and the introductions in which the males obtained a long-term stable position in the group.

\begin{tabular}{l|ll|lr|rr} 
Success & \multicolumn{2}{|c|}{ Introduction success (N=64) } & \multicolumn{2}{|c|}{$\begin{array}{l}\text { Long-term stability- } \\
\text { (success only) (N=49) }\end{array}$} & \multicolumn{2}{|c}{ Long-term stability (N=64) } \\
\hline Yes & 49 & $77 \%$ & 38 & $77 \%$ & 38 & $59 \%$ \\
No & 15 & $23 \%$ & 11 & $23 \%$ & 26 & $41 \%$
\end{tabular}

\section{Introduction success}

We composed three separate models to test for the effect of seasonality, male characteristics and group characteristics on introduction success. First, introductions right before or early in the breeding season appeared to be more successful than introduction late in- or outside the breeding season (McFadden $R^{2}=0.046, \chi^{2}=3.206$ ). Yet, delta $\mathrm{AlCc}<2$, indicating that the timing of an introduction is not a reliable predictor of success (Table A1 in Appendix 1). Second, a model including the male characteristics age, experience and body weight predicted introduction success best (McFadden $\mathrm{R}^{2}=0.190, \chi^{2}=13.226$, Table A2 in Appendix 1). Post-hoc analysis revealed that prime males were more successful than young males (McFadden $R^{2}=0.126, b=2.197$, $z=2.578$ ), that heavier males had a higher chance of success (McFadden $R^{2}=0.081$, $b=0.392, z=2.157)$, and that more experienced males tended to be more successful (McFadden $\mathrm{R}^{2}=0.061, \mathrm{~b}=1.346, \mathrm{z}=1.906$ ). However, there are no inexperienced young males in our dataset and young males are least heavy. Therefore, we ran the same analysis on a sample including prime and old aged males only. This results in a best predicting model with male age as the only predictor (McFadden $R^{2}=0.095, \chi^{2}=3.288$, Table A3 in Appendix 1). Additionally, when testing the effect of body weight on the success of young males only, the null model is the best predicting model (Table A4 in Appendix 1). Taken together, this indicates that there is no effect of experience or body weight, and age is the most important male characteristic predicting introduction success. Indeed, delta AICc $<1$ when removing these factors from our model (Table A2 in Appendix 1), implying no effect of experience and body weight on introduction success. We therefore consider the model with only age as a predictor the best predicting model, wherein young males are less successful than prime aged males (Figure 1). Third, in the model including group characteristics, the number of females in the group and the presence of pregnant females best predicted introduction success (McFadden $\mathrm{R}^{2}=0.176, \chi^{2}=12.255$, Table A5 in Appendix 1). Post-hoc analysis revealed that introductions into groups with more females (McFadden $R^{2}=0.039, b=-4.060$, 
$z=-1.945$ ) but without pregnant females (McFadden $R^{2}=1.116, b=2.038, z=2.921$ ) were most successful (Figure 2). Taken together, introductions of males that had reached at least prime age, into groups with more females but without pregnant females were most successful.

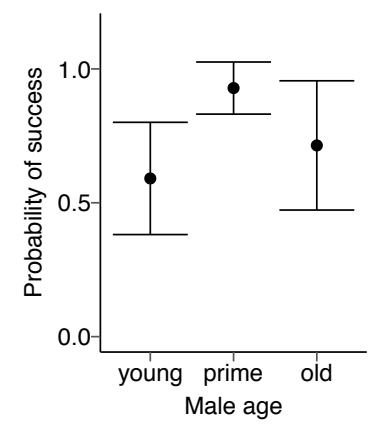

Figure 1 The effect of male age on introduction success (mean + 95\% confidence interval).
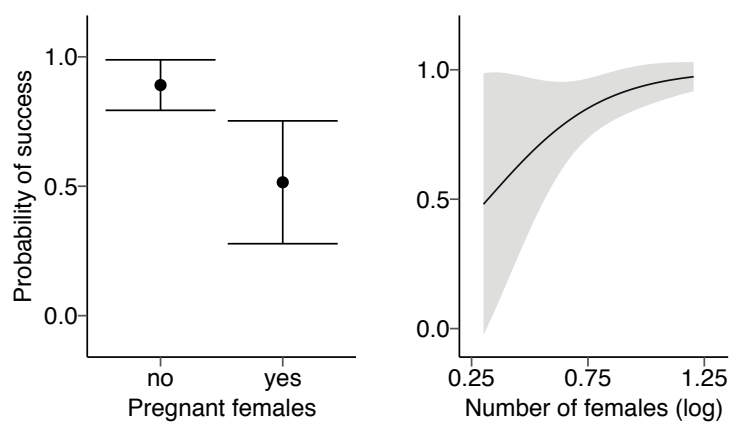

Figure 2 The effect of the presence of pregnant females (left) and the number of females (right) in the group on introduction success (mean $+95 \%$ confidence interval).

\section{Long-term stability}

Three separate models were composed to test the effect of seasonality, male characteristics and group characteristics on long-term stability (including both successful and unsuccessful introductions). First, seasonality did not affect long-term stability (McFadden $\mathrm{R}^{2}=0.015, \chi^{2}=1.282$ ), as the null model was a better predictor of stability than the model including the timing of the introductions (Table A6 in Appendix 1). Second, when focussing on male characteristics, long-term stability was best predicted by a model including male experience, the age a male was removed from his natal group, and body weight (McFadden $R^{2}=0.130, \chi^{2}=11.210$, Table A7 in Appendix 1). Post-hoc analysis revealed that introductions of males that were removed from their natal group at normal age were more often long-term stable than introductions with males that were removed from their natal group early (McFadden $R^{2}=0.051$, $b=-1.710, z=-2.407)$. Males that were removed late from their natal group did not differ from early removed males $(b=-0.658, z=-0.895)$ or males removed at normal age $(b=-$ $1.052, z=-1.364$ ) (Figure 3). The sample of males removed early from their natal group included peer-reared individuals, while the normal and late removed males concerned only group-reared individuals. To ensure the observed effect was not caused by peerrearing only, we compared the long-term stability of peer-reared individuals with that of group-reared individuals. Yet, the null-model is a better predictor of long-term stability than the model including peer-rearing (Table A8 in Appendix 1), implying that the observed effect of the age males were removed from their natal group is not 
caused by peer-rearing only. Introductions of heavier males were more long-term stable (McFadden $R^{2}=0.045, b=-0.288, z=-1.906$ ) (Figure 3). Male experience was not an important factor in the model, as delta $\mathrm{AIC} c<0.25$ when removing male experience from the model (Table A7 in Appendix 1). Finally, the model including group characteristics revealed that the number of matrilines and the presence of pregnant females in the group best predicted long-term stability (McFadden $R^{2}=0.083, \chi^{2}=7.177$, Table A9 in Appendix 1). Post-hoc analysis showed that introductions into groups with few matrilines (McFadden $\mathrm{R}^{2}=0.043, \mathrm{~b}=1.078, \mathrm{z}=1.937$ ) and without pregnant females (McFadden $R^{2}=0.038, b=1.065, z=1.826$ ) were more long-term stable (Figure 4). Delta AICc of the number of matrlines is close to 2 , indicating that this factor is important for the model, while the contribution of pregnancy to the model is less clear as AICc $\approx 1.4$ for this factor (Table A9 in Appendix 1). Taken together, introductions of heavy males that were not removed from their natal group at early age, into groups with few matrilines and without pregnant females were most likely to lead to long-term stability.

\section{Male experience}

Male experience as a breeding male does not automatically certify future performance. Of the introductions of experienced males $(N=27) 89 \%$ were successful, while $67 \%$ was long-term stable. Moreover, males that were unsuccessful or unstable after their first introduction ( $N=7$ ) were always successfully introduced into another group (100\%), and long-term stable after $71 \%$ of the introductions. Thus, a males' performance during previous introductions cannot be directly translated to future introductions.

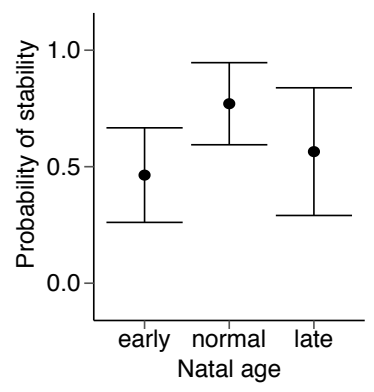

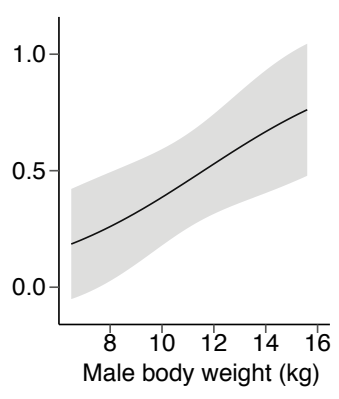

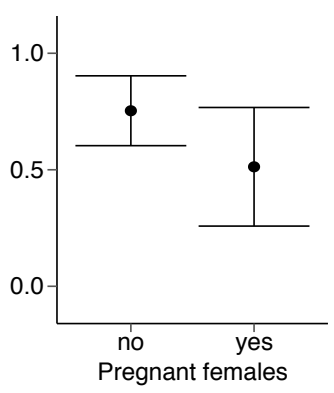

Figure 3 The effect of the age a male was removed from his natal group (left) and male body weight (right) on long-term stability (mean $+95 \%$ confidence interval).

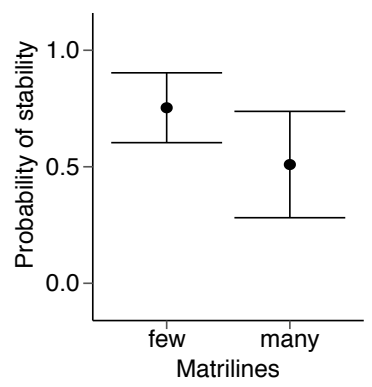

Figure 4 The effect of the number of matrilines (left) and the present of pregnant females in the group (right) on long-term stability (mean $+95 \%$ confidence interval). 


\section{Discussion}

This study aimed to identify the factors affecting introduction success and longterm group stability of male rhesus macaque introductions. Introductions of males that reached at least prime age into groups with more females but without pregnant females were most successful. Long-term stability was highest after introductions of heavy males that were removed from their natal group when they were at least 3.5 years old, into groups with few matrilines and without pregnant females.

\section{Introduction success and long-term stability}

Almost $77 \%$ of the studied introductions were successful, meaning that the male remained in the group full-time for at least four weeks. In the wild, males may fail to enter groups (Packer and Pusey 1979; van Noordwijk and van Schaik 1985); and unsuccessful immigration has even been reported in a wild group lacking adult males (Neville 1968). This shows that it is natural for males to fail in entering a group. However, it is not clear how high male immigration success in wild groups is. Male introduction success in captivity may vary from 0 to $100 \%$, possibly depending on the timing of the introduction (Bernstein et al. 1977). However, in this study (Bernstein et al. 1977) also the group composition differed between the successful and unsuccessful introductions: all introductions into female-only groups $(\mathrm{N}=6)$ were successful and occurred during the breeding season; while all introductions into groups of females with dependent infants $(\mathrm{N}=6)$ were unsuccessful and occurred outside the breeding season. In our study, group composition was more similar to that of the unsuccessful introductions, as the study groups consisted of adult females and their non-adult offspring. This indicates that $77 \%$ introduction success may be relatively high. Unfortunately, no other studies on male introduction success were found in literature. However, our view that $77 \%$ introduction success is relatively high is strengthened when comparing this to introduction success of pair-formation in macaques. Pairformation only concerns two individuals and is therefore socially less complex that introductions in a group. Different studies reported pair-formation in macaques with success rates varying from 40 to $100 \%$, with on average $85 \%$ success (Line et al. 1990; Crockett et al. 1994; Reinhardt et al. 1995; Doyle et al. 2010; Truelove et al. 2017; Worlein et al. 2017). This implies that introducing a new male into an existing social group, while carefully managing the introduction, is almost equally risky as forming pairs. Although the current introduction success is reasonably good, understanding what contributes to introduction success may improve husbandry procedures even further.

About $77 \%$ of the males that were successfully introduced obtained a long-term stable social position in their new group. In unstable groups, the males were removed due to within group aggression (i.e. either male-female or female-male aggression). Social instability is associated with stress and injuries through increased aggression levels (Sapolsky 1983, 1992; Shively et al. 1986; Crockford et al. 2008). Moreover, removing the male from a group due to social instability, will lead to another risky introduction. Therefore, assessing social instability is crucial when aiming to improve 
animal welfare. In wild long-tailed macaques, up to $28 \%$ of the males may leave their group within several months after successful group entry (van Noordwijk and van Schaik 1985, 2000). These numbers are difficult to translate to our study as not all wild males obtained the alpha position in their new group, and male-male competition may have been at play. However, it illustrates that it is not unnatural for males to fail to settle in their new group after successful entry. To our knowledge, we are the first to report this process in captivity through focussing on long-term stability after male introductions. Similarly, one study focussing on pair-formation showed that $94 \%$ of initially successful pairs was compatible (Truelove et al. 2017). Altogether, this indicates that not all animals may be compatible, even after successful introductions, and that this phenomenon is natural. Therefore, groups should be closely monitored, even after the successful introduction of a male.

\section{Timing}

Female receptiveness towards new males was expected to be higher right before and early in the breeding season; the time frame at which natural migration occurs (Lindburg 1969; Boelkins and Wilson 1972; Drickamer and Vessey 1973; Cheney and Seyfarth 1983; Mehlman 1986; Sprague 1992; Sussman 1992; Hayakawa and Soltis 2011). However, the timing of the introductions did not affect introduction success or long-term stability. This contrasts with previous research on rhesus macaque introductions (Bernstein et al. 1977) and implies that female receptivity for males does not affect female hostility towards unfamiliar males. The main difference between this previous study and our study is the composition of the groups. The groups in our study were naturalistic, consisting of different matrilines, while the previously published study described male introductions into groups of unrelated females. Moreover, they did not focus on the male's characteristics. Our results indicate that both male characteristics and the presence of matrilines are important for the outcome of introductions. Possibly, the new male's characteristics and the group composition are most important in determining introduction success and long-term stability, and may thereby overrule the effect of timing.

\section{Male strength}

Female resistance to new males may be less successful and cease sooner when stronger males are introduced. A male's body weight can be used as a measure for strength, as high body mass corresponds to fighting ability in wild male crested macaques (Marty et al. 2017). Heavier males in our study had higher long-term stability. This indicates that male strength plays a role during introductions. However, the relationship between male body weight and body condition in captivity is less straightforward than in the wild. Heavier males may be obese instead of stronger, as captive macaques may suffer from obesity (Bauer et al. 2011). Obese animals are less fit and may experience physical difficulties in conflicts with resident females. However, in our data set obesity is rare (BPRC data) and variability in body weight is likely more related to a male's strength. Therefore, our results imply that stronger males are better 
able to obtain and maintain a position in a new social group, and only strong males should be selected for introductions.

In the wild, male strength may be greatest when males are at prime age. Indeed, younger males were less successful during introductions than prime aged males, while old males did not differ from young or prime aged males. This shows that males should not be introduced before they reached full adult strength. However, old males were as successful as prime aged males, while their body condition may be deteriorating. Possibly, captive primates are able to maintain their strength for longer than their wild counterparts due to food abundance in captivity. Therefore, older males may still be strong and successful during introductions. Altogether, our results indicate that heavy, prime aged and old males should be selected for introductions to increase the chances of introduction success and long-term stability.

\section{Male social history and experience}

Males are expected to need social skills to obtain and maintain a position in a new group. Therefore, a male's social history and experience may affect introduction success and long-term stability. First, the age at which a male was removed from his natal group affected long-term stability, but not introduction success. Males removed from their natal group before the age of 3.5 years were able to initially enter a group, but often failed to establish a stable social position on the long-term. Removing animals from their natal group too early often leads to inadequate social behaviour (Bastian et al. 2003; Kempes et al. 2008; Veenema 2009). However, most studies reporting these effects compared peer-reared animals with animals growing up with their mother. Post-hoc analysis revealed that there was no difference in long-term stability between males that were peer-reared and male that grew up in naturalistic social groups. A statistical difference only emerged when taking the males removed from their natal group at early age together with the peer-reared males. Altogether, this indicates that it is important for males to be in their natal group at the onset of puberty. During this time, males may not only practice sexual behaviour, which drastically increases at puberty (Michael and Wilson 1973; Hanby and Brown 1974), but also learn social skills necessary to establish a stable social position in future groups. Therefore, males should stay in their natal group until they are at least 3.5 years old to fully develop their social behaviour. This corresponds to the age at which wild males naturally start migrating (Drickamer and Vessey 1973; Mehlman 1986; van Noordwijk and van Schaik 2000). The current norm for weaning at research facilities is generally between 10-14 months (Prescott et al. 2012). This weaning age should be drastically increased to allow the animals to fully develop their social skills, thereby improving animal welfare and decreasing the risks of male introductions.

The presence of natal males aged three or more during introductions did not affect introduction success and long-term stability. The natal males were likely too small to affect male-male competition with the introduced adult males, which is a great cost wild males experience during migration (Dittus 1975; Zhao 1994; Marty et al. 2017). 
Therefore, leaving males in their natal group until they are at least 3.5 years old will not affect future introductions into their natal group, while it improves the settlement of the males themselves in their future groups.

Second, a male's experience as a breeding male did not affect introduction success and long-term stability. In the wild, males may change groups several times in their lives (Sprague et al. 1998; Kuester and Paul 1999; van Noordwijk and van Schaik 2000). Males with experience in previous breeding groups could have gained social skills that enable them to better obtain a position in a new group. However, when selecting breeding males, their genetic representation in the breeding colony (Ballou et al. 2010) should be taken into account. Experienced males already have offspring in the breeding colony, and may therefore be less preferred males for reintroduction. Therefore, inexperienced males are often preferred. Moreover, males that failed during their first introduction (i.e. unstable or unsuccessful) did not automatically fail during their second introduction. Thus, if males with valuable (i.e. underrepresented) genetics fail during their first introduction, introduction into another group can still be considered.

\section{Group composition}

The composition of the group may affect a male's ability to enter a group in the wild. First, introductions into groups with more females were more successful, while there was no effect of the number of females on long-term stability. This effect is surprising, as wild males do not select their new group based on the number of females (Boelkins and Wilson 1972; Drickamer and Vessey 1973; Henzi and Lucas 1980; Cheney and Seyfarth 1983; van Noordwijk and van Schaik 1985; Marty et al. 2016). Moreover, female coalitions against new males (Packer and Pusey 1979; Cooper et al. 2001; Rox et al. 2018) can be larger in groups with more females. Therefore, introductions into larger groups were expected to be less successful. Possibly, since the number of matrilines is independent of the number of females in a groups, larger groups are more stable because they consist of larger matrilines. Female primates within a matriline form close social bonds (Silk 2002; Silk et al. 2006). These bonds may buffer stress during introductions (Crockford et al. 2008), and increase stability within a group (McCowan et al. 2018). Increased stability is, in its turn, associated with lower aggression levels from females to males (Shively et al. 1986), which may increase introduction success. Second, introductions into groups with fewer than four matrilines resulted in more long-term stable groups, than introductions into groups with many matrilines. Wild macaque groups may consist of 1-3 matrilines (de Ruiter and Geffen 1998; Larson et al. 2018), while groups with a large number of matrilines are unstable (Ehardt and Bernstein 1986; Oates-O'Brien et al. 2010). Males likely experience difficulties in obtaining a stable social position in a group when the relationships between resident individuals are already unstable. Overall, our results indicate that males should be introduced in groups with many females divided over few matrilines. To obtain such groups, females should remain in their natal group during their whole life to support the formation of large matrilines, similar to the wild. 


\section{Female reproductive state}

Female reproductive state was expected to affect a female's attitude towards new males, and thereby affect introduction success and long-term stability. Pregnant and lactating females were expected to show increased resistance during male introductions since they are at risk of infanticide (Beehner et al. 2005; Engh et al. 2006). Even though infanticide risk is generally low in rhesus macaques, infanticide has been observed in this species (Camperio Ciani 1984). Indeed, introductions into groups containing pregnant females were less successful, and less long-term stable. This indicates that infanticide risk can have long-lasting effects on female resistance to new males, which fits with the notion that males may commit infanticide until an infant no longer suppresses its mother's fertility (Camperio Ciani 1984; Crockett and Sekulic 1984; Winkler et al. 1984; Soltis et al. 2000). Therefore, also females with dependent infants at the start of the introduction may be at risk of infanticide when a new male enters a group (Beehner et al. 2005; Engh et al. 2006). However, the presence of infants did not affect introduction success or long-term stability. This contrasts with previous studies in species with high infanticide risk (Pereira and Weiss 1991; Ichino 2005; Engh et al. 2006). Possibly, part of the infants present during the introduction were at weaning age, and their mothers' fertility was no longer inhibited by the nursing. Male infanticide is only beneficial if a female becomes fertile sooner when her infant is killed (Hrdy $1977,1979)$, as would be the case for pregnant females. Therefore, the infanticide risk is higher for pregnant females compared to lactating females. Overall, introductions into groups containing pregnant females should be avoided, while the presence of lactating females does not affect the outcome of introductions.

In summary, necessary male introductions in naturalistic captive groups are potentially risky, and sometimes unsuccessful. Implementing information on the male's natural preference to enter specific groups and female resistance into captive introduction management can enhance introduction success and long-term stability. We show that carefully managed introductions are often successful and long-term stable. Introduction management can be further improved through only introducing males after they reached prime age. Moreover, introduced males should be heavy and should have remained in their natal group for at least 3.5 years. Groups should consist of few large matrilines, and preferably not contain pregnant females. Overall, the more closely the group and the introduction mimic natural migration patterns, the higher the chances of introduction success and long-term stability. This fits with the idea that animal welfare can be optimized when husbandry and group management remain close to the animal's natural situation. Therefore, naturalistic group housing and dynamics are important to ensure long-term stable captive primate groups. 


\section{Appendix 1}

Table A1 The delta AICc during stepwise backwards model selection of the logistic regression model on the effect of timing on introduction success.

\begin{tabular}{ll}
\multicolumn{2}{c}{ Step 1 } \\
\hline Excluded & $\boldsymbol{\Delta ~ A I C c}$ \\
\hline- & 0 \\
Timing & 1.206
\end{tabular}

Table A2 The delta AICc during stepwise backwards model selection of the logistic regression model on the effect of male characteristics on introduction success.

\begin{tabular}{ll|ll}
\multicolumn{2}{c|}{ Step 1 } & \multicolumn{2}{c}{ Step 2 } \\
\hline Excluded & $\boldsymbol{\Delta \text { AICC }}$ & Excluded & $\boldsymbol{\Delta}$ AICC \\
\hline Natal age & -1.754 & - & 0 \\
- & 0 & Age & 0.160 \\
Experience & 0.646 & Experience & 0.353 \\
Age & 0.626 & Body weight & 0.794 \\
Body weight & 1.556 & &
\end{tabular}

Table A3 The delta AICc during stepwise backwards model selection of the logistic regression model on the effect of male characteristics on introduction success, when only including introductions of prime and old males.

\begin{tabular}{ll|ll|ll|ll}
\multicolumn{2}{c|}{ Step 1 } & \multicolumn{2}{c|}{ Step 2 } & \multicolumn{2}{c|}{ Step 3 } & \multicolumn{2}{c}{ Step 4 } \\
\hline Excluded & $\boldsymbol{\Delta} \boldsymbol{A} / \boldsymbol{C} \boldsymbol{c}$ & Excluded & $\boldsymbol{\Delta} \boldsymbol{A} \boldsymbol{C} \boldsymbol{C c}$ & Excluded & $\boldsymbol{\Delta ~ A I C c}$ & Excluded & $\boldsymbol{\Delta} \boldsymbol{A} \boldsymbol{C} \boldsymbol{C}$ \\
\hline Natal age & -3.575 & Body weight & -0.338 & Experience & -0.297 & - & 0 \\
Body weight & -0.36 & - & 0 & - & 0 & Age & 1.289 \\
- & 0 & Experience & 0.365 & Age & 2.391 & & \\
Experience & 0.396 & Age & 1.664 & & & & \\
Age & 1.087 & & & & & &
\end{tabular}

Table A4 The delta AICc during stepwise backwards model selection of the logistic regression model on the effect of male characteristics on introduction success, when only including introductions of young males.

\begin{tabular}{ll|ll}
\multicolumn{1}{c|}{ Step 1 } & & \multicolumn{2}{c}{ Step 2 } \\
\hline Excluded & $\boldsymbol{\Delta} \boldsymbol{A I C \boldsymbol { C }}$ & Excluded & $\boldsymbol{\Delta} \mathbf{A I C C}$ \\
\hline Natal age & -1.692 & Body weight & -0.819 \\
- & 0 & - & 0 \\
Body weight & 0.378 & &
\end{tabular}


Table A5 The delta AICc during stepwise backwards model selection of the logistic regression model on the effect of female characteristics on introduction success.

\begin{tabular}{|c|c|c|c|c|c|c|c|}
\hline \multicolumn{2}{|c|}{ Step 1} & \multicolumn{2}{|c|}{ Step 2} & \multicolumn{2}{|c|}{ Step 3} & \multicolumn{2}{|c|}{ Step 4} \\
\hline Excluded & $\triangle A I C C$ & Excluded & $\triangle A I C C$ & Excluded & $\triangle A I C C$ & Excluded & $\triangle A I C C$ \\
\hline Natal males & -1.532 & Matrilines & -1.325 & Lactation & -0.223 & - & 0 \\
\hline Matrilines & -1.515 & Lactation & -0.125 & - & 0 & Females & 2.186 \\
\hline Lactation & -0.260 & - & 0 & Females & 2.390 & Pregnancy & 7.513 \\
\hline - & 0 & Females & 2.952 & Pregnancy & 7.128 & & \\
\hline Females & 2.402 & Pregnancy & 7.127 & & & & \\
\hline Pregnancy & 7.241 & & & & & & \\
\hline
\end{tabular}

Table A6 The delta AICc during stepwise backwards model selection of the logistic regression model on the effect of timing on long-term stability.

Step 1

\begin{tabular}{ll}
\hline Excluded & $\boldsymbol{\Delta A I C \boldsymbol { C }}$ \\
\hline Timing & -0.719 \\
- & 0
\end{tabular}

Table A7 The delta AICc during stepwise backwards model selection of the logistic regression model on the effect of male characteristics on long-term stability.

\begin{tabular}{ll|ll}
\multicolumn{2}{c|}{ Step 1 } & \multicolumn{2}{c}{ Step 2 } \\
\hline Excluded & $\boldsymbol{\Delta}$ AIC & Excluded & $\boldsymbol{\Delta ~ A I C C}$ \\
\hline Age & -3.281 & - & 0 \\
- & 0 & Experience & 0.427 \\
Experience & 0.139 & Body weight & 1.980 \\
Body weight & 1.298 & Natal age & 2.604 \\
Natal age & 1.571 & &
\end{tabular}

Table A8 The delta AICc during stepwise backwards model selection of the logistic regression model on the effect of peer-rearing on long-term stability.

\begin{tabular}{ll}
\multicolumn{2}{c}{ Step 1 } \\
\hline Excluded & $\boldsymbol{\Delta A I C c}$ \\
\hline Peer-rearing & -1.371 \\
- & 0
\end{tabular}


Table A9 The delta AICc during stepwise backwards model selection of the logistic regression model on the effect of female characteristics on long-term stability.

\begin{tabular}{ll|ll|ll|ll}
\multicolumn{2}{c|}{ Step 1 } & \multicolumn{2}{c|}{ Step 2 } & \multicolumn{2}{c|}{ Step 3 } & \multicolumn{2}{c}{ Step 4 } \\
\hline Excluded & $\triangle A I C C$ & Excluded & $\triangle$ AICC & Excluded & $\triangle A / C C$ & Excluded & $\triangle A I C C$ \\
Lactation & -1.945 & Natal males & -1.395 & Females & -0.944 & - & 0 \\
Natal males & -1.368 & Females & -0.608 & - & 0 & Pregnancy & 1.431 \\
Females & -0.529 & - & 0 & Pregnancy & 1.658 & Matrilines & 1.868 \\
- & 0 & Matrilines & 1.685 & Matrilines & 2.618 & & \\
Pregnancy & 1.605 & Pregnancy & 1.688 & & & & \\
Matrilines & 1.696 & & & & &
\end{tabular}





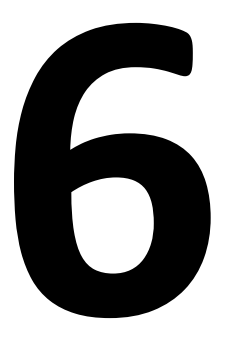

\section{Establishing new between-sex social bonds during male introductions in captive rhesus macaques}

Astrid Rox, Annet L Louwerse, Jan AM Langermans, Elisabeth HM Sterck 


\begin{abstract}
Many group living animals form close social bonds with their fellow group members. The existence of benefits of social bonding has been extensively studied. Yet, the mechanism through which new social bonds are established remains unknown. Therefore, this study focusses on the establishment of new female-male social bonds in captive rhesus macaques (Macaca mulatta) during four male introductions. Patterns in social behaviour and tolerance at the start of the introductions were compared to the social bonds established after the introductions. Females that tolerated their new male during the first five hours of contact showed aggression, affiliation, and mating with their new male during this time frame. Male-female affiliation and mating were exchanged with female-male aggression, and male-female affiliation was related to female-male affiliation. Especially females that received male affiliation and mated with their new male were likely to establish a social bond. A preliminary analysis one year after one of the introductions revealed that 2 of 4 established social bonds endured, and no new bonds were established. Altogether, our results imply that the initial interactions between unfamiliar individuals determine the establishment of social bonds. In particular, mating, reciprocation in affiliation, and possibly reconciliation of female-male aggression through mating and male affiliation may contribute. The female-male bonds formed during these early interactions can be long-lasting. Therefore, the initial interactions between unfamiliar individuals may have important long-term fitness consequences.
\end{abstract}

\title{
Introduction
}

Social bonds are common among group living animals, such as horses (Equus caballus: Cameron et al. 2009), goats (Capra aegagrus: Aschwanden et al. 2008), African elephants (Loxodonta africana: Archie and Chiyo 2012), spotted hyena's (Crocuta crocuta: Smith et al. 2011), and raven (Corvus corax: Braun and Bugnyar 2012). The existence of social bonds is particularly well-described in primates, who form complex social relationships with their group members (reviewed in: Silk 2002; Massen et al. 2010; Seyfarth and Cheney 2012). Especially female-female bonds have been extensively studied (e.g. Henzi and Barrett 1999; Hohmann et al. 1999; Cords 2002; Silk et al. 2006, 2010a; Langergraber et al. 2009), but also female-male (e.g. Hill 1990; Hohmann et al. 1999; Lemasson et al. 2008; Langergraber et al. 2013; Massen and Sterck 2013; Haunhorst et al. 2016), and male-male (e.g. Mitani 2009; Berghänel et al. 2011; Ostner and Schülke 2014; Young et al. 2014) bonds are common in primates. Primates with close social bonds are more likely to support each other in conflicts (Manson 1994; Berghänel et al. 2011; Carne et al. 2011; Young et al. 2014; Haunhorst et al. 2017); often resulting in a higher dominance rank (van Schaik et al. 2004; Schülke et al. 2010; Gilby et al. 2013). In addition, social bonds can act as a buffer to stress, making animals less responsive to socially stressful situations (Beehner et al. 2005; Crockford et al. 2008; Wittig et al. 2008). Moreover, animals may protect individuals with whom they have close bonds from danger (Cords 2002) and harassment (Manson 1994; Beehner et al. 2005; Lemasson et al. 2008; Haunhorst et al. 2017). Finally, 
animals with more social bonds often have higher reproductive success (Silk et al. 2003, 2009; Schülke et al. 2010; Massen et al. 2012; Mcfarland et al. 2017), and may live longer (Silk et al. 2010b). Thus, it is clear that primates commonly form close social bonds with their group members and these bonds can increase an individual's fitness.

In contrast to the existence and benefits of social bonds, little is known about the mechanisms through which social bonds are established in non-human primates. The start of social bonding is occasionally studied in humans. However, the establishment and maintenance of social bonds in humans is often studied using questionnaires (e.g. Ladd 1990; Wentzel and Erdley 1993; Hollingsworth and Buysse 2009), ignoring the behavioural mechanisms underlying social bonding. Moreover, one known mechanism through which children form bonds, simply asking other children to be their friend and then play with them as if they are friends (Danby et al. 2012), is a unlikely mechanism for social bonding in non-human primates. However, the behavioural mechanisms through which social bonds are established in non-human primates is not yet studied. Therefore, the behavioural mechanism that underlies social bonding in primates remains unknown.

The need to establish new social bonds comes with the arrival of new individuals in a group through birth or immigration. In many primate species, including macaques (Macaca spp.), males migrate to new groups several times in their lives; a possible mechanism against inbreeding (Dittus 1975; Greenwood 1980; van Noordwijk and van Schaik 1985; Zhao 1994). Consequently, unfamiliar adult males enter existing social groups, resulting in the need to establish new female-male and male-male bonds. Social bonding between females and new males may start soon after a new male entered a social group (Samuels and Altmann 1986; Fragaszy et al. 1994; Cooper et al. 2001). Social bonding could help males to successfully enter a new group (Yamada 1971; Bernstein et al. 1977; Wilson and Gordon 1979; Lewis 2008; Kawazoe and Sosa 2019), and may therefore have important fitness consequences. Yet, how these bonds are formed remains unknown. When infants are born into a group, they particularly establish good social bonds with individuals they interact with early in life (Dunayer and Berman 2017). A similar mechanism may be at play during the establishment of bonds between females and a new male. Some females may tolerate a new male soon after his group entry and spend time in his proximity, while other females refrain from associating (Rox et al. 2018). Females that tolerate a new male soon, may interact with him more often, which possibly leads to the establishment of a social bond.

Additionally, the behaviour individuals use to establish social bonds is unclear. Since primate social bonds are commonly measured using proximity and grooming levels between individuals (Hohmann et al. 1999; Mitani 2009; Silk et al. 2009; Langergraber et al. 2009; Schülke et al. 2010; Massen et al. 2012; Massen and Sterck 2013), the common view is that social bonds form through affiliation. Indeed, newborn infants are likely to establish social bonds with individuals that display affiliative behaviour, including grooming, embracing and cuddling, towards them (Dunayer and Berman 2017). Receiving these behaviours has been linked to the release of oxytocin (Crockford et al. 2013), a neurotransmitter and hormone that plays an important role in attachment 
and pair-bond formation in voles (Microtus spp.) (reviewed in: Young et al. 2008). Moreover, the release of oxytocin promotes affiliative behaviour (Campbell 2008; Ragen and Bales 2013). Consequently, animals that show affiliation may receive affiliation or tolerance in return (Henzi and Barrett 1999; Carne et al. 2011; Xia et al. 2013). Thus, reciprocity in affiliative behavior and tolerance may lead to social bonds (Xia et al. 2013), as is also indicated by the EMO-model (i.e. an agent based model on the emergence of social bonds in primate groups) (Evers et al. 2015). In bonobos (Pan paniscus) and Assamese macaques (Macaca assamensis), reciprocity in grooming is indeed higher between more closely bonded individuals (Hohmann et al. 1999; Haunhorst et al. 2016). Thus, females that tolerate new males early and affiliate with him soon after his group entry may establish female-new male bonds through reciprocity.

Another behaviour that several researchers linked to the establishment of social bonds is mating (Samuels and Altmann 1986; Sprague 1992; Cooper et al. 2001; Rox et al. 2018). Females may groom a new male after mating to secure protection for themselves and their future offspring (Beehner et al. 2005; Sonnweber et al. 2015), services that males may provide to females with whom they have a close social bond. Additionally, consort formation after mating has previously been proposed as an important factor in the formation of social bonds in savanna baboons (Papio cynocephalus anubis) (Bercovitch 1991), and social bonds more often follow than precede mating in chacma baboons ( $P$. ursinus) (Baniel et al. 2016). Finally, females that mate with a new male soon after his group entry may establish a social bond with him soon (Samuels and Altmann 1986; Cooper et al. 2001; Hayakawa and Soltis 2011), while social bonds between non-receptive females and new males may emerge later (Cooper et al. 2001). Since female mating interest in new males is generally high (Bercovitch 1991; Manson 1995; Inoue and Takenaka 2007; Rox et al. 2018, 2019a), mating may play an important role in the formation of female-male social bonds.

Lastly, also aggression has been linked to social bonds. Female-new male aggression levels are usually high during male group entry (Bernstein et al. 1977; Rox et al. 2018). Females may particularly show aggression towards males they do not like, implying that low aggression levels may be linked to the establishment of social bonds. However, one study reported that especially females that recently attacked a new male started grooming him (Kawai 1960). Similarly, animals that groom each other more often may also show more aggression towards each other than animals that spend less time together (Carne et al. 2011; Crofoot et al. 2011). Although counterintuitive, high levels of aggression may be exchanged with affiliation through reconciliation. Reconciliation is common among primates and occurs more often between closely bonded animals (Aureli and Cords 2000). Reconciliation may increase the strength of a bond and repair valuable social bonds after aggression (Koyama 2001). Possibly, reconciliation may also increase the strength of newly emerging social bonds. Yet, a link between reconciliation of aggression and the establishment of social bonds has never been studied. High aggression levels could underlie the establishment of social bonds, as long as this aggression is reconciled. 
Taken together, researchers have suggested several mechanisms through which social bonds can be established. Yet, the process of social bonding has never been studied. Therefore, this study aims to identify the mechanism through which femalenew male social bonds are formed, by studying four male introductions in captive groups of rhesus macaques (Macaca mulatta). These introductions mimic the natural migration patterns of rhesus macaques and offer the opportunity to study the establishment of new social bonds in detail. Although rhesus macaque groups are female-bonded, adult males play an important role in the group and are usually well integrated into the social network (Hill 1990). We therefore expect multiple females in each group to establish a social bond with their new male during the introductions. The established social bonds are measured right after an introduction is finished. We predict that the initial interactions between the females and the new males at the start of the introductions determine which females establish a social bond. Females that tolerate their new male in their proximity at the start of the introduction are expected to engage in affiliation, mating, and aggression with their new male, which may leads to the establishment of new social bonds. Finally, we assess whether the social bonds established after the introductions are still present one year later, based on data of one of the four groups.

\section{Methods}

\section{Subjects and Housing}

The study was conducted at the Biomedical Primate Research Centre (BPRC) in Rijswijk, the Netherlands. We studied 45 adult female rhesus macaques from four different captive groups (the Liby group, the Marieke group, the Clio group, and the Mayke group). Group sizes varied from 5 to 16 adult females ( $\geq 3$ years of age) and their non-adult offspring. Natural migration patterns are mimicked in the groups. Male offspring are removed from the groups when they are approximately 4 years of age, female offspring remain in their natal group for life. Non-natal breeding males are replaced about every 4 years, to prevent inbreeding. Four of such necessary management procedures were followed during this study. The new breeding males were experienced (i.e. they were the breeding male in another group at the BPRC prior to the studied introduction) and ranged in age from $9.6-13.4$ years. The males were unfamiliar and unrelated to individuals in their new group.

The animals were housed in spacious indoor ( $72 \mathrm{~m}^{2}, 2.85 \mathrm{~m}$ high) and outdoor (208 $\mathrm{m}^{2}, 3.1 \mathrm{~m}$ high) enclosures, connected through elevated corridors. The enclosures contained multiple elevated beams and environmental enrichment items (Vernes and Louwerse 2010).

\section{Introduction Process}

One adult male rhesus macaque was introduced to each of the subject groups, referred to as introduction $A$ (Liby group), introduction $B$ (Marieke group), introduction $\mathrm{C}$ (Clio group), and introduction $\mathrm{D}$ (Mayke group). The introductions were part of the 
regular BPRC management procedures and followed the BPRC introduction guidelines (Rox et al. 2018, 2019). The males were gradually introduced into their new social groups. First, the males could only access the females through wired mesh. When aggression levels were low, the males were physically introduced to the females. They spent part of their day with the females, while they were housed in an enclosure adjacent to the females during the remainder of the day and at night. Contact possibilities and time of contact were progressively increased. The males were allowed to stay with the group full-time after respectively 12 (introduction A), 14 (introduction B), 20 (introduction C), and 47 (introduction D) days of the contact. Experienced animal caretakers managed the introductions, deciding when to increase contact time and contact possibilities.

\section{Behavioural Observations}

Data were collected between December 2014 and May 2015 (introductions A, B, and C), and between February and May 2016 (introduction D). All aggressive and affiliative behaviour between the resident females and their new male was recorded during continuous focal sampling of the male. Additionally, the time the females spent in proximity (i.e. within $1 \mathrm{~m}$ ) of their new male, and all mating between the subjects and their male were scored.

Behavioural observations were carried out from the first day of physical contact up until 2 weeks after the completion of the introduction (i.e. when the male was allowed to remain in the group full-time). During the introductions, observations were carried out whenever the male spent time with the group. When the male was allowed to stay with the group full-time, observations took place on workdays between 9 am and 12 am, and between $1 \mathrm{pm}$ and $4 \mathrm{pm}$. Video recordings of the new male were used to complement the behavioural observations at the start of the introductions, until the frequency of interactions decreased to the point the observer was able to record all interactions in real time. When the animals had access to the inside and outside enclosures, the location of the observations followed a semi-random balanced (i.e. $50 \%$ inside, $50 \%$ outside) schedule. The time each subject spent inside or outside was recorded to determine the time the individual was visible to the observer.

Finally, the animals in introduction A were observed for two weeks exactly one year after the end of the introduction. The observation method was the same as the method used right after the introduction.

\section{Measures}

This study compared behaviour from two different time periods: the start of the physical introduction; and the period after completion of the introduction. The start of the introduction concerns the first five hours the male had physical access to the resident females. During this period, the animals were locked outside during all four introductions, so that all interactions between the resident females and the new male were observed. The time period after the introduction concerns the first two weeks 
the male was allowed to remain in the group full-time. The animals had access to both their inside and outside enclosures during this period.

It is likely crucial for females to tolerate the new male in her proximity to bond. Therefore, we estimated female tolerance of a new male at the start of the introduction (cf. Rox et al. 2018). Tolerating females either accepted approaches by their new male, approached their new male non-aggressively themselves, spent time in his proximity, or engaged in grooming with him. We determined for each female whether or not she tolerated her new male early during the introduction. Early tolerance between a dyad occurred when a female started tolerating their new male during the first five hours of the introduction.

The established social bonds were measured during the first two weeks after the introductions, based on the time a female spent in proximity of the new male. Females were in proximity of the male when they were within $1 \mathrm{~m}$ of the male, this includes being/sitting together, but also interactions such as grooming or mating. A majority (66\%) of the female-male dyads spent less than $2 \%$ of their time in each other's proximity. Female-male dyads that spent at least $2 \%$ of their time together were more distinct (Figure 1). Therefore, we only consider females that spent at least $2 \%$ of their time in proximity of the male as socially bonded with their new male. The same method was used to determine the existence of social bonds between the new male and the females one year after introduction $A$.

To analyse which behaviours were correlated to early tolerance and the establishment of social bonds, data on the occurrence of female-male aggression, male-female aggression, female-male affiliation, male-female affiliation and mating were transformed to binary data. This was necessary as there were too many zeroes in our sample, caused by the lack of (certain) interactions in many female-male dyads. 0 indicated the absence of a certain interaction in a dyad, while a 1 indicated a certain interaction did occur in a dyad. This was indicated for both time frames (start of the introduction, and after the introduction) separately. Note that the presence of early tolerance at the start of the introduction (yes/no) and the establishment of social bonds after the introduction (yes/no) were already binary variables.

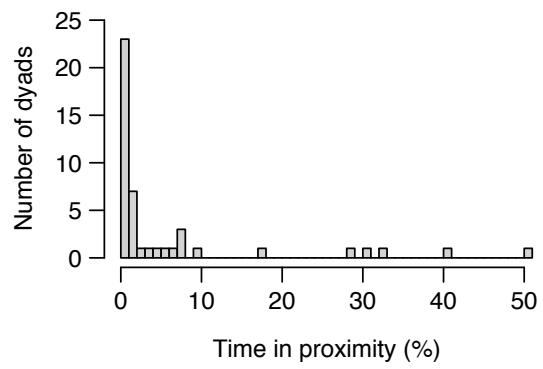

Figure 1 A histogram showing the relation between the number of female-male dyads and the time they spent in proximity during the first two weeks after the introductions. 
Finally, we combined different types of behaviours (i.e. female aggression, male aggression, female grooming, male grooming, mating) together in a single variable that represents the number of different types of interactions in a dyad. First, we counted in how many different types of interactions each female-male dyad engaged, varying from 0 to 5 . Second, we composed a combined variable from the occurrence of mating and male-female affiliation, to test whether animals that engaged in both these behaviours early in the introduction had higher chances of establishing social bonds than females that engaged in only one of these interactions, varying from 0 to 2 . Therefore, we scored for each female whether she engaged in none of these interactions early in the introduction, engaged in only one of these interactions, or in both. Females that engaged in mating only $(\mathrm{N}=2)$ were grouped with the females that received affiliation only $(N=4)$, due to the low number of females that engaged in only one of these interactions.

\section{Data analysis}

First, Kruskal-Wallis teste were used to test for differences in behaviour during the four introductions. As the tests revealed no significant differences in female-male aggression, male-female aggression, female-male affiliation, male-female affiliation and mating between the introductions (all $p \geq 0.47$ ), data from the four introductions were grouped for analysis. Still, introduction ID was added to all models to ensure the observed effects do not differ between the introductions.

Logistic regression models and $\mathrm{Chi}^{2}$ tests were used to analyse the binomial data. The first logistic regression model tested whether early tolerance was related to the establishment of social bonds, with the establishment of social bonds as dependent variable, early tolerance as predictor variable, and introduction ID as covariate. Second, we tested whether early tolerance predicted the occurrence of interactions between the females and their new male using logistic regression models. Therefore, the occurrence of female-male aggression, male-female aggression, female-male affiliation, male-female affiliation or mating was added as the dependent variable, each to a separate model. Early tolerance was added as predictor variable to each of these models, and introduction ID as a covariate. Third, we tested the relation between all the behaviours whose early occurrence was significantly predicted by early tolerance, using $\mathrm{Chi}^{2}$ tests. $\mathrm{Chi}^{2}$ tested were used because the behaviours occurred in the same time frame (i.e. the first 5 hours of the introductions), making it impossible to determine which behaviour would be the dependent variable and which behaviour would be the predictor variable in a model. Fourth, logistic regression models were run to determine whether the occurrence of a certain behaviour during the first five hours of contact predicted the occurrence of the same behaviour after the introduction. Therefore, the occurrence of either female-male aggression, male-female aggression, female-male affiliation, male-female affiliation or mating after the introduction was added as the dependent variable, while the occurrence of the same behaviour at the start of the introduction was added as predictor variable. Again, a separate model was 
run for each behaviour and introduction ID was added as covariate. Finally, we tested the relation between the behaviour early during the introduction and the establishment of social bonds. The same was done for the behaviour that occurred in the two weeks after the introduction. Therefore, the establishment of social bonds was added as dependent variable to logistic regression models. The occurrence of female-male aggression, male-female aggression, female-male affiliation, male-female affiliation, and mating, the combined occurrence of mating and male-female affiliation, and the number of different interactions a dyad engaged in during the first five hours of the introduction or after the introduction were added as the dependent variable, with a separate model for variable and introduction ID as a covariate. Post-hoc Tukey testing was used for the logistic regression model on the effect of the combined occurrence of mating and male-female affiliation to identify which categories (none, only one behaviour, both behaviours) significantly differed from each other.

$R$ version 3.2.3 was used for statistical analysis and composing figures, using the packages multcomp, effects, jtools, and ggplot2. Testing was two-tailed and significance level was set to $p \leq 0.05$. All $p$-values were corrected for multiple testing using Bonferroni correction.

\section{Results}

\section{Establishment of social bonds}

In total, 16 of the 45 females had established a social bond after the introduction with their new male. This concerned 4 out of the 16 females (25\%) in introduction A, 6 out of the 13 females (46\%) in introduction B, 4 out of the 12 females (33\%) in introduction C, and 2 out of 5 females (40\%) in introduction D. Possibly, the interactions between females and their new male are important determinants of the establishment of these social bonds. Indeed, females that tolerated their new male during the first five hours of the introductions were more likely to establish a social bond with their new male (binary logistic regression: $N=45, \chi^{2}=11.859, p=0.002$, Figure 2 ). There was no significant difference between the introductions $(p=1.000)$.

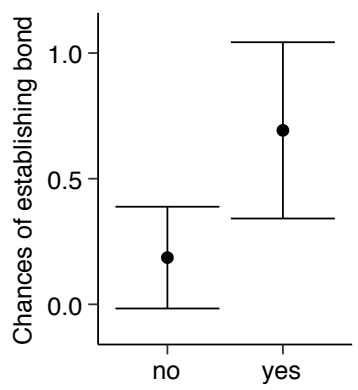

Early tolerance
Figure $\mathbf{2}$ Early tolerance of a new male by a female significantly increases the chance she will establish a social bond with the new male. 


\section{Early tolerance and behaviour}

As a first step in determining how the early tolerating females establish social bonds with their new male, we studied the interactions between early tolerating females and their new male. Early tolerating females were more likely to engage in female-male aggression (binary logistic regression: $N=45, \chi^{2}=7.477, p=0.004$ ), female-male affiliation (binary logistic regression, $N=45, \chi^{2}=16.474, p<0.001$ ), male-female affiliation (binary logistic regression: $\mathrm{N}=45, \chi^{2}=14.736, \mathrm{p}<0.001$ ), and mating with their new male (binary logistic regression: $N=45, \chi^{2}=38.871, p<0.001$ ) during the first five hours of the introductions than females that did not tolerate their new male during that time (Table 1). Male-female aggression during the first five hours of contact was independent of early tolerance (binary logistic regression: $N=45, \chi^{2}=6.955, p=0.365$; Table 1 ). There were no significant differences between the introductions during any of the analyses (all $p \geq 0.205$, Table 1). Thus, females that tolerate the male early on engage in a variety of interactions with their new male, and these interactions may contribute to the establishment of social bonds.

Table 1 The effect of early tolerance on male-female and female-male on the establishment of social bonds at the end of the introductions interactions during the first five hours of contact. Significant $p$-values $(p \leq 0.05)$ are indicated in bold.

\begin{tabular}{lllll} 
Dependent variable & Independent variable & $\mathbf{C h i}^{\mathbf{2}}$ & $\mathbf{p}$-value & $\begin{array}{l}\text { Introduction differences } \\
\text { (p-value) }\end{array}$ \\
\hline Social bonds & Early tolerance & 11.859 & $\mathbf{0 . 0 0 2}$ & 0.493 \\
Female-male aggression & Early tolerance & 7.477 & $\mathbf{0 . 0 0 4}$ & 0.205 \\
Male-female aggression & Early tolerance & 6.955 & 0.365 & 1.000 \\
Female-male affiliation & Early tolerance & 16.474 & $\mathbf{< 0 . 0 0 1}$ & 1.000 \\
Male-female affiliation & Early tolerance & 14.736 & $\mathbf{0 . 0 0 1}$ & 1.000 \\
Mating & Early tolerance & 38.871 & $\mathbf{< 0 . 0 0 1}$ & 1.000
\end{tabular}

\section{Relationship between early behaviours}

Second, we studied whether females that engaged in one early behaviour were also more likely to engage in other types of interactions with their new male at the start of the introductions. Females-male aggression was not related to female-male affiliation (Chi ${ }^{2}$ test: $N=45, \chi^{2}=5.409, p=0.080$ ). Yet, females that displayed female-male aggression were more likely to receive male-female aggression (Chi ${ }^{2}$ test: $N=45, \chi^{2}=7.386$, $\mathrm{p}=0.026)$, receive male-female affiliation ( $\mathrm{Chi}^{2}$ test: $\left.\mathrm{N}=45, \chi^{2}=6.856, \mathrm{p}=0.035\right)$ and mate with their new male ( $\mathrm{Chi}^{2}$ test: $\left.\mathrm{N}=45, \chi^{2}=10.355, \mathrm{p}=0.005\right)$. Next, male-female aggression was not related to female-male affiliation (Chi ${ }^{2}$ test: $N=45, \chi^{2}<0.001$, $\mathrm{p}=1.00$ ), male-female affiliation (Chi ${ }^{2}$ test, $\mathrm{N}=45, \chi^{2}=1.412, \mathrm{p}=0.940$ ), or mating (Chi ${ }^{2}$ test: $\left.N=45, \chi^{2}=1.999, p=0.628\right)$. However, females that affiliated with their new male were more likely to receive male-female affiliation (Chi ${ }^{2}$ test: $N=45, \chi^{2}=11.845, p=0.002$ ) and mate with him (Chi ${ }^{2}$ test: $N=45, \chi^{2}=14.957, p<0.001$ ). Finally, females that mated 
with their new male were also more likely to received male-female affiliation (Chi ${ }^{2}$ test: $\left.\mathrm{N}=45, \chi^{2}=26.910, p=0.003\right)$. Thus, once a female interacts with her new male, she is likely to engage in different kinds of interactions with her new male during the first five hours of the introductions (Table 2). These behaviours may have long-term consequences.

Table 2 The relationship between the behaviours that were linked to social tolerance during the first five hours of the introductions. Significant $p$-values $(p \leq 0.05)$ are indicated in bold.

\begin{tabular}{llll}
$\mathbf{1}^{\text {st }}$ variable & $\mathbf{2}^{\text {nd }}$ variable & $\mathbf{C h i}^{\mathbf{2}}$ & $\mathbf{p}$-value \\
\hline Female-male aggression & Male-female aggression & 7.386 & $\mathbf{0 . 0 2 6}$ \\
Female-male aggression & Female-male affiliation & 5.409 & 0.080 \\
Female-male aggression & Male-female affiliation & 6.856 & $\mathbf{0 . 0 3 5}$ \\
Female-male aggression & Mating & 10.355 & $\mathbf{0 . 0 0 5}$ \\
Male-female aggression & Female-male affiliation & $<0.001$ & 1.000 \\
Male-female aggression & Male-female affiliation & 1.412 & 0.940 \\
Male-female aggression & Mating & 1.999 & 0.628 \\
Female-male affiliation & Male-female affiliation & 11.845 & $\mathbf{0 . 0 0 2}$ \\
Female-male affiliation & Mating & 14.957 & $<\mathbf{0 . 0 0 1}$ \\
Male-female affiliation & Mating & 26.910 & $\mathbf{0 . 0 0 3}$
\end{tabular}

\section{Long-term correlates of early behaviour}

Next, we tested whether the behaviours displayed during the first five hours of contact are reliable predictors of the female-male interactions on the long term. Females that displayed female-male aggression during the first five hours of the introductions, were not more likely to display female-male aggression after the introduction (binary logistic regression: $N=45, \chi^{2}=9.742, p=0.970$ ). Neither did malefemale affiliation predict the occurrence of male-female affiliation (binary logistic regression: $N=45, \chi^{2}=2.738, p=1.000$ ), or predicted the occurrence of mating during the first five hours whether a female mated with the male after the introduction (binary logistic regression: $\mathrm{N}=45, \chi^{2}=2.038, \mathrm{p}=0.970$ ). In contrast, females that received malefemale aggression at the start of the introductions were more likely to also receive aggression after the introductions (binary logistic regression: $N=45, \chi^{2}=8.219, p=0.035$ ). Additionally, the occurrence of female-male affiliation significantly predicted the occurrence of female-male affiliation after the introductions (binary logistic regression: $\left.\mathrm{N}=45, \chi^{2}=9.304, p=0.045\right)$. There were no significant differences between the introductions in any of these analysis ( $p \geq 0.225$, Table 3 ). Overall, the occurrence of 2 out of the 5 studied behaviours at the start of the introductions predicted the occurrence of the same behaviour on the long-term. 
Table 3 The relationship between the behaviours displayed during first five hours of the introductions (f) and the same behaviours after the introductions (a). Significant $p$-values $(p \leq 0.05)$ are indicated in bold.

\begin{tabular}{lllll} 
Dependent variable & Independent variable & $\mathbf{C h i}^{\mathbf{2}}$ & $\mathbf{p}$-value & $\begin{array}{l}\text { Introduction } \\
\text { differences } \\
\text { (p-value) }\end{array}$ \\
\hline Female-male aggression (a) & Female-male aggression (f) & 9.742 & 0.970 & 0.225 \\
Male-female aggression (a) & Male-female aggression (f) & 8.219 & $\mathbf{0 . 0 3 5}$ & 1.000 \\
Female-male affiliation (a) & Female-male affiliation (f) & 9.304 & $\mathbf{0 . 0 4 5}$ & 1.000 \\
Male-female affiliation (a) & Male-female affiliation (f) & 2.738 & 1.000 & 1.000 \\
Mating (a) & Mating (f) & 2.038 & 0.970 & 1.000
\end{tabular}

\section{Early and late behaviour and social bonds}

Finally, we tested whether the behaviours females displayed early in the introduction determined whether they established a social bond after the introduction with their new male. Females that engaged in more different types of interactions with their new male at the start of the introductions were most likely to establish a social bond (binary logistic regression: $\mathrm{N}=45, \chi^{2}=10.604, \mathrm{p}=0.015$; Figure 3 ). When looking into the behaviours in more detail, especially females that received male-female affiliation (binary logistic regression: $N=45, \chi^{2}=9.600, p=0.035$ ) and mated with their new male (binary logistic regression: $\mathrm{N}=45, \chi^{2}=9.298, \mathrm{p}=0.045$ ) established social bonds (Figure 3, Table 4). Especially females that engage in both of these behaviours may establish a social bond with their new male. Yet, females that received both male affiliation and mated with him were not more likely to establish a social bond than females that engaged in only one of these interactions. Even though the model predicted the establishment of social bonds significantly (binary logistic regression: $N=45, \chi^{2}=8.518$, $\mathrm{p}=0.035$ ), post-hoc testing revealed no significant differences (post-hoc Tukey correction: $z=-0.558, p=0.841$ ). Additionally, female-male aggression (binary logistic regression: $\mathrm{N}=45, \chi^{2}=7.582, \mathrm{p}=0.090$ ), male-female aggression (binary logistic regression: $N=45, \chi^{2}=3.007, p=0.182$ ), and female-male affiliation (binary logistic regression: $\left.N=45, \chi^{2}=5.119, p=0.475\right)$ did not affect the establishment of social bonds (Table 4).

Finally, female-male affiliation after the introduction particularly occurred between females that established a social bond with their new male (binary logistic regression: $\mathrm{N}=45, \chi^{2}=25.685, p<0.001$ ), while male-female aggression after the introduction was not related to the established social bonds (binary logistic regression: $N=45, \chi^{2}=3.300$, $p=0.316)$. Altogether, females that engage in more different types of interactions with the male at the start of the introductions, and particularly engage in mating or receive male affiliation, are likely to establish social bonds with their new male (Table 4, Figure 4). 

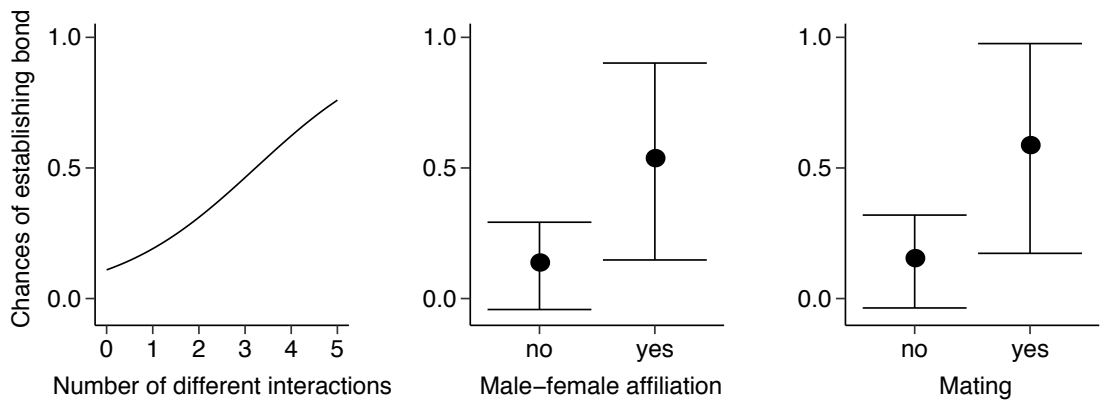

Figure 3 Females that engaged in more different types of interactions with their new male during the first five hours of contact (left), females that received male-female affiliation during that time frame (middle), or engaged in mating with their new male (right) were more likely to establish a social bond.

Table 4 The relationship between the behaviours displayed during first five hours of the introductions (f), their long-term correlates ( $a$ ) and the bonds established after the introductions. Significant $p$-values $(p \leq 0.05)$ are indicated in bold.

\begin{tabular}{lllll} 
Dependent variable & Independent variable & Chi $^{\mathbf{2}}$ & p-value & $\begin{array}{l}\text { Introduction } \\
\text { differences } \\
\text { (p-value) }\end{array}$ \\
\hline Social bonds & Number types of interactions (f) & 10.604 & $\mathbf{0 . 0 1 5}$ & 1.000 \\
Social bonds & Female-male aggression (f) & 7.582 & 0.090 & 1.000 \\
Social bonds & Male-female aggression (f) & 3.007 & 0.182 & 1.000 \\
Social bonds & Female-male affiliation (f) & 5.119 & 0.475 & 1.000 \\
Social bonds & Male-female affiliation (f) & 9.600 & $\mathbf{0 . 0 3 5}$ & 1.000 \\
Social bonds & Mating (f) & 9.298 & $\mathbf{0 . 0 4 5}$ & 1.000 \\
Social bonds & Male-female affiliation + mating (f) & 8.515 & $\mathbf{0 . 0 3 5}$ & 1.000 \\
Social bonds & Male-female aggression (a) & 3.300 & 0.316 & 1.000 \\
Social bonds & Female-male affiliation (a) & 25.685 & $\mathbf{0 . 0 0 1}$ & 1.000
\end{tabular}

\section{Durability of social bonds}

For introduction A, data were collected on the established social bonds one year after the introduction. Two of the 16 females showed a social bond with their male one year after the introduction. These females were two of the four females that had established a social bond with their male after the introductions. Thus, 50\% of the bonds established during the introduction endured until at least one year after the introduction. Moreover, no new bonds emerged that were not yet present directly after the introduction. Unfortunately, sample size is insufficient to conduct statistics or draw hard conclusions on the durability of the bonds that are established during introductions. 


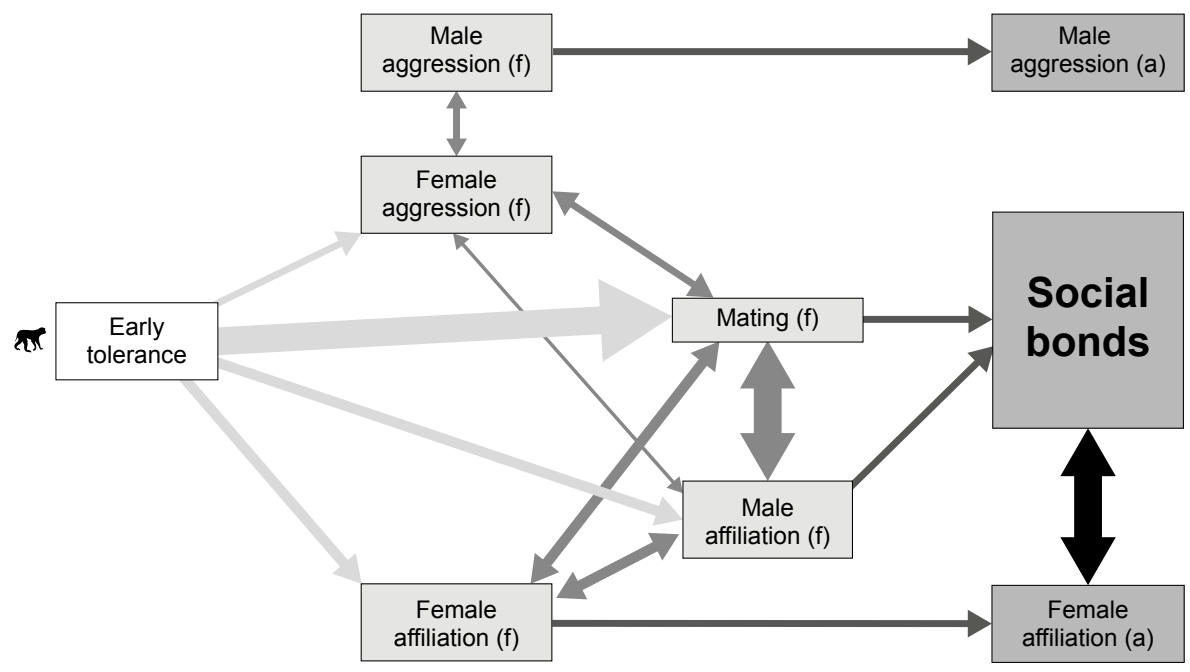

Figure 4 An overview of the effect of early tolerance (white) on the behaviour at the start of the introduction (light grey, f), and the relation between these behaviours and the behaviour after the introductions (a), including the establishment of social bonds (dark grey). Significant relations between the behaviours are indicated by the arrows, the thickness of the lines represents the strength of the relationship (i.e. $\chi^{2}$ from the logistic regression models). The darkness of the boxes and arrows indicat the time frame during which the behaviours occurred, varying from white at the start of the introduction, to dark grey at the end of the introduction.

\section{Discussion}

This study aimed to identify the mechanisms through which female-male social bonds are formed by studying four male introductions in captive groups of rhesus macaques. We explored female-male and male-female interactions during the first five hours of contact, as we expected the initial interactions to be important determinants of the establishment of social bonds. Our results show that females that tolerated their new male early, were more likely to engage in female-male aggression, female-male affiliation, male-female affiliation and mating during the first five hours of contact. Females that engaged in more different interactions with their new male during these first five hours were more likely to have established a social bond after the introduction. In particular, females that received male affiliation and mated with their new male were most likely to establish a social bond. These results show that the initial interactions between individuals are indeed linked to the formation of social bonds, and that affiliation and mating may play an important role in establishing social bonds. 


\section{Early tolerance}

We expected that initial interactions between females and their new male were important in the establishment of social bonds. Indeed, females that tolerated their new male during the first five hours of the introductions were more likely to establish a social bond. This is in line with the only known study reporting on the formation of social bonds in which it is shown that infants born into a social group particularly form bonds with individuals that associate with them early in life (Dunayer and Berman 2017). This strengthens the view that early interactions are important in establishing social bonds. Moreover, female tolerance is determined based on proximity measures (see Rox et al. 2018). Proximity is often used as a measure of bond strength in studies on primates (Hohmann et al. 1999; Mitani 2009; Langergraber et al. 2009; Schülke et al. 2010; Massen and Sterck 2013; Baniel et al. 2016; Haunhorst et al. 2016) and other species (goats: Aschwanden et al. 2008, horses: Cameron et al. 2009, spotted hyenas: Smith et al. 2011). It is therefore not surprising that animals that tolerate each other in their proximity are likely to establish bonds. Females may take more initiative for proximity than new males do during introductions (unpublished data, Chapter 7). This implies that female behaviour plays an important role in the establishment of social bonds. Yet, being in proximity may be a passive behaviour; animals may just tolerate each other to be near but not interact actively. Therefore, tolerance itself is likely not the behaviour used to actively establish social bonds, yet, it likely facilitates interactions between animals. These interactions may form the basis of social bonds.

\section{Affiliative behaviour}

Social bonds are characterized by high levels of affiliative behaviour between the individuals (e.g. Silk et al. 2009; Massen and Sterck 2013). Therefore, affiliative behaviour between the females and their new male at the start of the introduction was expected to increase bond formation. Indeed, male-female affiliation was related to the establishment of social bonds. This is in line with previous research, where newborn infants are likely to establish social bonds with individuals that display affiliative behaviour towards them early in life (Dunayer and Berman 2017). Yet, female-male affiliation did not directly affect bond formation. Still, females that received affiliation from their new male were more likely to show affiliation towards him. Additionally, female-male affiliation at the start of the introduction predicted the occurrence of female-male affiliation after the introduction, which was, in its turn, related to the established social bond. Taken together, this may imply that male affiliation is reciprocated by female affiliation, which is linked to social bonding through male affiliation on the short-term, and female affiliation on the long-term. Thus, reciprocation of male-female affiliation may underlie bond formation. This is in line with the results of the EMO-model, an agent based model on the establishment of social bonds in primates (Evers et al. 2015). Additionally, reciprocation of affiliative behaviour has been observed in several species (reviewed in: Freidin et al. 2017), and may be higher in individual with close social bonds (Hohmann et al. 1999; Haunhorst et al. 2016). The hormone and neurotransmitter oxytocin may be the physiological mechanism behind 
the reciprocation of affiliation. Receiving affiliation increases an individual's oxytocin levels, leading to an increased tendency to show affiliation (Regan and Kitchener 2005; Campbell 2008). Thereby, oxytocin may lead to the reciprocation of affiliation and underlie bond formation. The role of oxytocin in intrasexual pair-bond formation is well known in vole species (Young et al. 2008). A similar mechanism may be at play in primates (Crockford et al. 2013). Overall, early tolerance between a female and a new male may lead to male and female affiliation, which may be reciprocated and underlie the formation of social bonds.

\section{Mating}

Females that mated with their new male at the start of the introduction were expected to be more likely to establish a social bond with their new male. Indeed, mating was linked to bond formation. This fits with previous research that suggested that females that mate with new males may build social bonds during group entry (Samuels and Altmann 1986; Cooper et al. 2001; Hayakawa and Soltis 2011). Moreover, this is in line with previous research showing that mating generally precedes bond formation, instead of bonding preceding mating (Baniel et al. 2016). Some researchers may consider mating a male-guided behaviour. Yet, the female role in mating should not be underestimated in macaques. Female macaques commonly invite new males to mate, rendering forced mating rare (unpublished data, Chapter 5). Additionally, primates females can successfully reject male mating attempts (Bercovitch 1995; Arlet et al. 2007; Overduin-De Vries et al. 2012; Rox et al. 2018). Males also can initiate and refuse female mating attempts (e.g. Overduin-De Vries et al. 2012). This shows that mating is a mutual behaviour, with two actively involved partners. Therefore, both males and females may initiate bonding through mating.

Females may groom new males after mating to secure protection for themselves and their future offspring (Sonnweber et al. 2015). In particular, male friends may protect females and their offspring from infanticide (Palombit et al. 2001; Beehner et al. 2005). This is in line with the observed exchange between female-male affiliation and mating, and the clear link between mating and the established social bonds. Yet, also male affiliation is exchanged with mating. Possibly, males use affiliation to gain mating access (Massen et al. 2012; Kulik et al. 2012) and thereby build social bonds with the females. Since mating rates between females and new males are generally high (Bercovitch 1991; Manson 1995; Inoue and Takenaka 2007; Rox et al. 2018, 2019a), mating may be one of the most important behaviours used to establish social bonds after a new male enters an existing group.

\section{Aggression}

Aggression was expected to be linked to bond formation. Yet, neither male-female aggression nor female-male aggression at the start of the introduction predicted the establishment of social bonds. Additionally, male-female aggression was not linked to early tolerance or any other behaviours displayed at the start of the introduction, except female-male aggression. This implies no link between male-female aggression and bond 
formation. In contrast, early tolerating female were more likely to display female-male aggression. This fits with previous studies in primates showing that aggression levels may be high between individuals that spend more time together (Carne et al. 2011; Crofoot et al. 2011). Additionally, female-male aggression was exchanged with mating and male-female affiliation at the start of the introductions. This fits with previous research reporting that especially females that attacked new males also engaged in grooming with him (Kawai 1960), yet, this concerned female-male grooming, in contrast to male-female affiliation in our study. Positive social behaviours, such as mating and male-female affiliation, may be used to reconcile after conflicts between the females and their new male (Aureli and Cords 2000). However, whether mating and affiliation indeed follow female-male aggression, a condition necessary for reconciliation, remains to be studied. Still, a link between reconciliation and bonds formation would not be surprising, as especially individuals with good social bonds reconcile (Aureli and Cords 2000). Additionally, the uncertainty reduction hypothesis on the possible function of reconciliation states that individuals may reconcile to decrease uncertainty about a relationship between individuals (Aureli and Schaik 1991). The uncertainty about a relationship will be especially high between unfamiliar individuals that are not yet socially bonded but do spent time in each other's proximity. Therefore, reconciliation can be expected between females and their new male at the start of the introduction, using behaviours linked to bond formation to reconcile. This also fits with our results indicating that dyads that engage in more different interactions (e.g. aggression and affiliation) are more likely to establish social bonds than females that engage in affiliation, aggression, or mating only. Thus, female-male aggression may be linked to bond formation through reconciliation, yet, there is no direct effect of female-male and malefemale aggression on the establishment of social bonds.

\section{Stability of social bonds}

We measured the establishment of social bonds over a relatively short time-span, varying from 12-47 days of contact (see Methods). Primates form long-lasting bonds with same-sex and opposite-sex group members (Hohmann et al. 1999; Silk et al. 2006, 2010a; Mitani 2009; Massen and Sterck 2013; Baniel et al. 2016). We cannot show conclusively that the bonds formed during the introductions are such long-lasting social bonds. Yet, there are indications that the bonds found directly after the introduction are indeed long-lasting. Our preliminary analysis on the presence of social bonds one year after one of the introductions showed that no new social bonds emerged, and two of the four bonds established after the introductions remained. Additionally, previous studies reported that the social bonds formed during male group entry can be strong (Fragaszy et al. 1994), and persist for at least six months (Samuels and Altmann 1986). Altogether, this strengthens our view that the initial interactions between individuals are important in establishing long-lasting social bonds.

However, not all females that established a social bond at the end of the introduction also maintained this bond. Our sample size on the long-term stability of social bonds is limited, but may provide valuable information for future analysis. The two females that 
maintained their bond were the two highest ranking females in the group. Several studies report that especially high ranking primate females form strong social bonds with males (Palombit et al. 2001; Archie et al. 2014; Baniel et al. 2016). Males may therefore focus their affiliation more on these females, as they may not have sufficient time to maintain bonds with many females in their group. This rank preference may be less apparent during the introductions compared to one year later, as the males may not be familiar with the female dominance hierarchy in their new group yet. Moreover, early tolerance, an important factor in the establishment of social bonds, is not affected by female dominance rank (Rox et al. 2018). Alternatively, we may need to be more conservative in our cut-off point for labelling a dyad socially bonded. We used data on the four introductions and saw a clear difference in the number of females that spent less than $2 \%$ of their time with their new male, and the number of females that spent more than $2 \%$ of their time with their new male (Figure 1). We therefore considered females that spent more than $2 \%$ of their time with their new male as socially bonded. The females we considered socially bonded in introduction A spent respectively 5.3\%, $7.5 \%, 19.3 \%$, and $27.2 \%$ of their time in proximity with their new male. The two females that spent most time with their new male maintained their social bond. Thus, longlasting social bonds may be formed during the introductions, yet, out dataset is too limited to draw decisive conclusions. Moreover, what exactly determines which bonds are long-lasting needs to be established in the future.

\section{Translation to other bonds and species}

Our results provide insights in the mechanism through which female-male social bonds are formed in rhesus macaques. However, this mechanism may differ between groups, between the types of bonds that are formed, between captive and wild groups, and between species. The question therefore remains how our results can be generalized to bond formation under different conditions or in different species. Our analysis revealed no differences in the mechanisms through which social bonds are formed between the introductions. This implies there is no effect of individual characteristics of the males, or the size or composition of the social groups on the mechanisms through which bonds are formed. Moreover, our results fit with previous studies, on different species, reporting the emergence of new social bonds between females and new males (Samuels and Altmann 1986; Cooper et al. 2001; Hayakawa and Soltis 2011). However, these studies only report with whom social bonds are formed (i.e. sexually receptive females), while details on the mechanism through which social bonds are established are lacking. The only known study focussing on the mechanism of bond formation showed that initial interactions between individuals affects the development of social bonds. Individuals that affiliated with infants when they were young and dependent on their mother were most likely to have established a social bond with the infant when it was more independent of the mother (Dunayer and Berman 2017). This implies that the mechanisms through which these bonds are formed may be similar to the mechanism found in our study. Additionally, manu researchers study the presence of social bonds within a wide array of species and in 
different environments using the same behaviours we found to affect the establishment of social bonds; namely affiliation and proximity (Hohmann et al. 1999; Beehner et al. 2005; Aschwanden et al. 2008; Cameron et al. 2009; Braun and Bugnyar 2012; Baniel et al. 2016; Haunhorst et al. 2016). This implies that early proximity between individuals and affiliative behaviour may determine the establishment of different kinds of social bonds in different species. Sexual behaviour or mating may only be used in the establishment of social bonds between adult males and females, yet mating can also be considered a positive social interaction between individuals of the opposite sex. Altogether, the mechanism through which female-male social bonds are established in our study may be widespread among the animal kingdom. Yet, more research, on a wide array of species is needed to confirm our findings.

\section{Conclusion}

In summary, females that tolerate new males early in their group engage in interactions important for the formation of social bonds. They receive more male affiliation and engage more in mating early during the introductions and these positive social behaviours are linked to the establishment of social bonds. The more types of interactions males and females engage in during the first hours of contact, the more likely they are to establish a social bond. Especially female reciprocation of male affiliation, and possibly reconciliation after female aggression though mating and maleaffiliation, may be important in bond formation. Thereby, social bonding is a joined effort, wherein both the new male and the female play an active role. Females may tolerate new males and seek proximity to them early during the introductions. Males may affiliate with some of these tolerating females they particularly like, and mate with them. Taken together, the initial positive social interactions between unknown individuals can have important, long-term, consequences for both males and females. Whether and how individuals interact at the start, may determine the establishment of enduring social bonds, and thereby affect their fitness. 



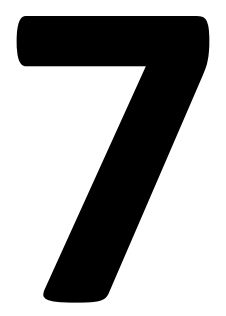

\section{Female rhesus macaques use mating tactics to regulate female access to a newly introduced male}

Astrid Rox, Annet L Louwerse, Jan AM Langermans, Elisabeth HM Sterck 


\begin{abstract}
Females often invest more time and energy in their offspring than males. Consequently, only males are expected to compete over mating access and employ mating tactics. Yet, there is emerging evidence that also females can compete over mating partners. However, it remains unclear which mating tactics females can employ and whether female-female competition indeed leads to increased mating access. Therefore, this study aims to identify whether females use mating tactics and link these tactics leads to increased mating access and reproductive success. This is studied during four male introductions in captive rhesus macaque (Macaca mulatta) groups, creating one-male groups. If females can employ mating tactics, they are expected to use them in this highly competitive situation. Indeed, females that approached their new male more often, and groomed him longer had higher mating access. Moreover, females started more female-female aggression on days they were sexually interested in their new male, and especially targeted other sexually interested females. Females that started more female-female aggression had higher mating access, while female dominance rank did not affect mating. Finally, there was no link between any female mating tactic and female reproductive success. Thus, females can use several mating tactics in a highly competitive situation, namely expressing their preference for a male through approaches and grooming, and expressing female competition through aggression, even though this does not increase reproductive success. These results challenge the common believe that only males employ mating tactics and compete over mates. Likely, mating tactics can be flexibly used by both sexes, depending on the social context.
\end{abstract}

\title{
Introduction
}

In many animal species, females invest more time and energy into their offspring than males (Trivers 1972; Smuts and Smuts 1993). This difference is especially apparent in mammals, where females have obligate care for their offspring during gestation and lactation. As a result, females prefer to mate with high quality males to ensure the best chances of survival for their offspring. In contrast, male fitness can be increased by a high quantity of mates (Trivers 1972; Smuts and Smuts 1993). Consequently, males may fight over access to females (Clutton-Brock et al. 1979; Mainguy et al. 2008) or may use other mating tactics to secure mating access, as is often observed in primates living in multi-male multi-female groups (Clutton-Brock and Parker 1995; Bercovitch 1997; Smuts and Smuts 1993; Bissonnette et al. 2011; Massen et al. 2012). In this, we define a mating tactic as any interaction between animals of the same (e.g. male-male aggression) or opposite sex (e.g. male-female grooming) leading to increased mating access. Females are not believed to employ mating tactics but may exert mate choice and prefer to mate with particular males.

Females may prefer to mate with males who can provide valuable protection for them and their future offspring (Clutton-Brock and McAuliffe 2009; Rebout et al. 2017). Moreover, males providing more paternal investment, which may coincide with friendships in primates, are often preferred mates (Clutton-Brock and McAuliffe 2009; 
Massen et al. 2012; Städele et al. 2019). Finally, females may prefer mating with new males that recently entered their group (Pereira and Weiss 1991; Inoue and Takenaka 2007; Hayakawa and Soltis 2011; Rox et al. 2018). Once a female prefers a certain mating partner, she may frequently approach him and initiate sexual interactions (Bercovitch 1991; Soltis et al. 1997; Setchell 2005; Arlet et al. 2007). However, the expression of female mate choice through approaches and initiation of sexual interactions may be overruled by male mating tactics (Soltis et al. 2001; Rebout et al. 2017). Therefore, it remains unclear under which circumstances females can express mate choice and increase the chances of mating with their preferred male (Soltis et al. 1997, 2001). When mating with preferred males provides monopolisable benefits, such as protection and paternal care, females can be expected to express mate choice (i.e. actively approach and invite males to mate) and increase mating access to their preferred partner by employing mating tactics.

Once a female approaches her preferred mating partner, she may groom him to gain mating access. Males can build long-term grooming relationships with females, so-called friendships, to gain mating access (Massen et al. 2012; Ostner et al. 2013; Städele et al. 2019) but also female-male grooming is common among primates (O'Brien 1991; Massen and Sterck 2013). Females may even play a more active role in female-male friendships than males, as changes in these friendships may result from a change in female rather than male behaviour (Palombit et al. 2001). Females can benefit from friendships when receiving male services, such as increased protection after mating (Palombit et al. 1997; Engh et al. 2006). Female-male grooming may be linked directly to mating, as especially females that engage in mating groom males (Samuels and Altmann 1986; Cooper et al. 2001; Arlet et al. 2007). Additionally, females may especially build long-term grooming relationships with dominant males, who are generally preferred mating partners (O'Brien 1991; Palombit et al. 2001; Arlet et al. 2007). Another study even used female-male grooming as a proxy for female sexual interest (Bercovitch 1991). Altogether, this implies that females may build friendships with males to gain mating access. However, the use of female-male grooming as a female mating tactic has not yet been studied.

Next to interacting with males to obtain mating access, females may also compete with other females over preferred mating partners. Therefore, females can be expected to use their dominance position to gain mating access, similar to primate males (Cowlishaw and Dunbar 1991; Berard 1999; Rodriguez-Llanes et al. 2009; Alberts 2012; Massen et al. 2012). Indeed, dominant females have higher reproductive success than subordinates (Whitten 1983; Harcourt 1987; Borries et al. 1991; van Noordwijk and van Schaik 1999; Pusey 2012; Liu et al. 2018). Yet this may be a consequence of priority of access to food leading to increased fertility or offspring survival rather than increased mating access. However, there is also evidence that female dominance leads to priority of access to males. In seasonal breeding species, dominant females may mate earlier than subordinates, indicating that dominant females can limit mating access of subordinates (Whitten 1983). Additionally, dominant females have more and stronger friendships with males than subordinate females. As there is only a limited number of 
males per group, females are thought to use their dominance to obtain access to males (Palombit et al. 2001; Archie et al. 2014). In yellow baboons (Papio cynocephalus), however, female dominance rank was not related to sexual consort formation (Bercovitch 1991). Taken together, the link between female dominance rank and mating access remains unclear.

If female dominance rank is not sufficient to exclude other females from mating with a preferred male, females may use female-female aggression to obtain mating access (Dunbar and Sharman 1983; Archie et al. 2014). Female baboons (Papio spp.) and redfronted lemurs (Eulemur rufifrons) are less likely to reproduce when there are more females in the group (Dunbar and Sharman 1983; Kappeler and Fichtel 2011). This suggests that females compete over mating and may prevent other females from reproducing, by preventing access to males, due to high costs of female-female fights (Kappeler and Fichtel 2011). Moreover, female long-tailed macaques (Macaca fascicularis) avoid male and female bystanders during mating, indicating that other females may disturb their mating attempts (Overduin-de Vries et al. 2013). Finally, especially reproductively active females engage in high levels of female-female aggression in baboons. Female-female aggression increased when there were more females with sexual swellings (i.e. a sign of fertility), and especially swollen females were the target of female-female aggression (Huchard and Cowlishaw 2011; Baniel et al. 2018). In addition, cycling females are more likely to be injured than pregnant or lactating females (MacCormick et al. 2012). Altogether, this implies that females may use female-female aggression to gain access to males. Yet, it remains unclear whether females gain mating access or other reproductive benefits (cf. male-male aggression; review: Alberts 2012) from engaging in female-female aggression.

Male behaviour may decrease the effectiveness of female mating tactics. Primate males are generally larger and stronger than females (Mittermeier et al. 2013; Rowe and Myers 2016). Therefore, they may overrule female mate choice and force females to mate using their strength or aggression (Smuts and Smuts 1993; Clutton-Brock and Parker 1995; Soltis et al. 1997). Such male coercion may especially overrule female mate choice when sexual dimorphism is high, decreasing the opportunities for female mating tactics. However, even highly sexually dimorphic baboon females may successfully reject a male's attempt to mate (Bercovitch 1995). Therefore, also females from sexually dimorphic species may employ mating tactics.

Taken together, there are indications that females may use female-male approaches, female-male grooming, their dominance, and female-female aggression as a mating tactics. Yet this has never been studied in detail. Moreover, it remains unknown whether females employing these mating tactics actually gain reproductive benefits. Therefore, this study aims to identify whether females use mating tactics, and whether these tactics yield reproductive benefits. This is studied during four male introductions into naturalistic captive rhesus macaque (Macaca mulatta) groups, creating one-male groups. During the introductions, there is limited and monopolizable access to a highly preferred mating partner, leading to a highly competitive situation. Thereby, we created circumstances where females are mostly expected to compete for mating. 
Additionally, rhesus macaques are seasonal breeders where reproductive synchrony between the females enhances female-female competition over males. The studied introductions are necessary management procedures for captive groups, and provide us with the opportunity to study female mating tactics in naturalistic primate groups from an experimental point of view. The situation is extreme but not unnatural. Macaques naturally live in multi-male multi-female groups, yet one-male groups have been observed (Neville 1968; van Noordwijk and van Schaik 1985; Ménard and Vallet 1993; Keane et al. 1997). All other group-settings, including group composition, female philopatry, and male migration patterns mimicked nature as closely as possible (see Rox et al. 2019), making it possible to translate the results to wild groups.

We expect females interested in mating with their new male to actively seek contact, and therefore approach him often and invite him to mate by presenting their hind quarters. Females that approach and present to their new male more often, are expected to gain more mating access, will be more likely to become pregnant, and give birth earlier than females that do not approach or present often. Similar results are expected for females that groom their new male longer, females with higher dominance ranks, and females that engage more in female-female aggression. The new males will have plenty of opportunity to mate, and will not need to pursue reluctant females to mate. Therefore, the males are not expected to employ any mating tactics.

\section{Methods}

\section{Subjects and housing}

The study was conducted in the rhesus macaque (Macaca mulatta) breeding colony of the Biomedical Primate Research Centre (BPRC) in Rijswijik, the Netherlands. Subjects were 43 adult females (age $\geq 3$ years) and four adult males. The females were part of four different social groups; the Liby group (introduction A), the Marieke group (introduction $\mathrm{B}$ ), the Clio group (introduction C), and the Mayke group (introduction D). The males were each introduced into one of these social groups. The groups varied in size from five to sixteen adult females, and their non-adult (age $<3$ years) offspring (five-ten individuals). Three females in the Clio group were pregnant at the start of the introduction, these females were not included in our analysis. In addition, the Marieke group contained two natal sub-adult males (age 3.5 years). All female subjects ( $N=43$ ) were potentially fertile (i.e. not on contraceptives and not pregnant) and ranged in age from 3.5 to 19.4 years. The introduced males were experienced breeding males (i.e. they were the breeding male in another group at the BPRC prior to the studied introduction) ranging in age from $9.6-13.4$ years. The males were unfamiliar and unrelated to individuals in their new group. The introductions were part of the colony management procedures at the BPRC. Natural migration patterns are mimicked in the BPRC breeding colony, meaning that females are philopatric and males are removed from their natal group when they reach sexual maturity. The groups contain one adult breeding male that is replaced every four to five years to prevent inbreeding. 
The animals were housed in spacious inside (72 $\mathrm{m}^{2}, 2.85 \mathrm{~m}$ high) and outside (208 m², $3.1 \mathrm{~m}$ high) enclosures, and could move freely between these enclosures outside introductory events. Both enclosures contained several environmental enrichment items, including elevated beams, firehoses, and a swimming pool (Vernes and Louwerse 2010).

\section{Introduction procedure}

The introductions followed the BPRC introduction guidelines and were conducted by specialized animal caretakers. The introductions took place during the 2014/2015 (introductions A, B and C) and 2015/2016 (introduction D) breeding seasons (i.e. between October and March). After familiarization with the group through wired mesh, the new male was physically introduced into his new group. The time the male spent with the group each day increased gradually, until he was allowed to stay in the group full-time. How fast an introduction progressed depended on the behaviour of the animals and estimations of introduction progress by the experienced animal caretakers. The four studied new males were allowed to remain in their group full-time after 12 (introduction A), 14 (introduction B), 20 (introduction C), and 47 (introduction D) days of contact with the females. For a detailed description of the BPRC introduction procedure see (Rox et al. 2018, 2019).

\section{Behavioural observations}

Before the introductions started, two weeks of baseline data were collected in the groups. During these observations, there was no adult male present. The new male was housed in an enclosure adjacent to the females, separated by a concrete wall. All occurrences of female-female conflicts and submission were recorded on eight days between $9 \mathrm{am}$ and $12 \mathrm{am}$, and between $1 \mathrm{pm}$ and $4 \mathrm{pm}$.

During the introductions, continuous focal observations were conducted on the new male, focussing on the interactions between him and the females. First, all mounts and copulations between the new male and the females, from now on labelled mating, were recorded. For each mating interaction, we indicated whether the interaction was voluntary or forced (i.e. the male used force or aggression to prevent a female from rejecting his mating attempt). Second, we scored mating invitation from the females to the new male through recording female presenting. Third, we noted all aggression, approaches (only introduction A, C, and D) and grooming time between the females and the new male and vice versa. Simultaneously, we recorded all occurrences of female-female conflicts. Finally, displacements and bared teeth between all individuals in the group were scored ad libitum to reconstruct the dominance hierarchy, using Matman (de Vries 1998). All groups showed a significant linear dominance hierarchy (Liby group: linearity index $h^{\prime}=0.96$, Marieke group: linearity index $h^{\prime}=0.85$, linearity index Clio group: $h^{\prime}=0.80$, Mayke group: linearity index $h^{\prime}=1$, all $\left.p<0.05\right)$.

Behavioural observations were carried out from the first day a male was physically introduced into the group, until 2 (introductions A \& C) or 4 (introduction B \& D) weeks after a male was allowed to stay with the group full-time. Observations took place 
whenever the male had physical access to the group. When the male was allowed to stay in the group during the entire workday or longer, observations were carried out between 9am and 12am, and between 1pm and 4pm. In total, 48 (introduction A), 95 (introduction B), 66 (introduction C), and 185 (introduction D) hours of observational data were collected.

\section{Measures}

Before the analysis started, the rate/h of all behaviours were calculated. The behaviour rates during the baseline period were calculated based on the time each female was visible to the observer. The rate of female-new male and new-male female interactions during the introductions, and the percentage of time grooming were calculated based on the time the new male was visible to the observer. We used the rates/ $h$ at which individuals started and received (i.e. was the target of) conflicts as a measure for aggression. Moreover, for part of our analysis, the occurrence of femalenew male grooming was transformed to binary data. Zero indicates no female-new male grooming occurred during the introduction and one implied grooming occurred at least once in a dyad. Similar binary transformations were applied to the occurrence of new male-female grooming and of mating. Next, we determined for each female during which introduction days she was sexually interested in the new male. We considered females sexually interested on the days they either presented to, did not reject sexual investigation by, or mated with their new male during the introduction. They were not sexually interested on all other introduction days. Finally, we calculated the change in female-female aggression started and received by subtracting the rate of female-female aggression during the baseline observations from the female-female aggression rates on the days a female was sexually interested. When correlating female-female mating competition to mating, mating rates were calculated based on the observation time on the days a female was sexually interested.

To estimate the effect of female and male mating tactics on female reproduction, we determined whether a female gave birth within one year after the start of the introduction. Moreover, the timing of giving birth was determined, where the timing concerned the order in which females gave birth, with the first female in a group numbered 1. Finally, we created categories of female dominance rank, to be able to combine the data of the four groups when analysing reproduction. The females within each group were divided in three equal parts based on rank, distinguishing high, middle and low ranked females.

\section{Statistics}

Kruskall-Wallis tests were used to identify possible differences in behaviour rates between the introductions. There were no differences in the rates of female-new male and new male-female interactions between the introductions, therefore, we grouped the data from all four introductions for analysis. Still, whenever it was possible to run models on our data, we added group ID as a factor to these models. 
First, we studied female initiative for contact by comparing female-new male and new-male female approach rates using Wilcoxon signed-rank test, and correlating female-new male approaches and female-new male presenting with mating using Spearman rank correlation tests. Data on approaches were available for three of the four introductions (A, C, and D), concerning 30 females.

Second, the link between female-new male grooming and mating was studied. As a first step in this analysis, we tested whether mating particularly occurred between dyads that groomed. This was necessary as there were relatively many females that did not engage in mating or grooming with their new male. We ran a binary logistic regression model with the binary occurrence of mating as the dependent variable, the binary occurrence of female-new male grooming as the predictor variable, and group ID as a covariate. Next, we used a generalized mixed model with a separate slope for each introduction to test whether females that groomed the male longer also had higher mating access. Therefore, data of the 25 females that groomed their new male at least once were selected. The percentage of time the females groomed their new male and the rate/h of female-new male mating were transformed using square root transformation. Mating rate/h was the dependent variable in the model, while femalenew male grooming was the predictor variable. Lastly, we investigated a possible direct link between female-new male grooming and mating. Therefore, we compared the time each female spent grooming the new male between days they were sexually interested and days they were not interested in their new male. Only data from females that groomed and mated with their new male at least once could be included in this analysis.

Third, the effect of female dominance on mating was tested using Spearman rank correlation tests. This was done for each of the introductions separately, as dominance rank is group-specific.

Fourth, we studied the effect of female-female aggression on mating. The rates at which each female started and received female-female aggression were compared between the baseline observations, days she was sexually interested, and days she was not sexually interested in the new male using Friedman tests. 37 females showed sexual interest in their new male at least once and could be included in this analysis. Finally, we tested whether the change in female-female aggression started or received was related to mating access, using Spearman rank correlation tests.

Fifth, we tested the occurrence of male mating tactics. Therefore, the occurrence of voluntary and forced mating was investigated. Moreover, Spearman rank correlation tests were used to correlate the rate/h of male initiated conflicts against a particular female with mating, female-new male approaches, and female-new male presenting. Next, the relationship between new male-female grooming and mating was tested similarly to the previous described method to test for a relationship between female grooming and mating, using binary logistic regression and a linear mixed model. However, a log transformation was used on the data entered into the linear mixed model, instead of a square root transformation. Finally, we compared the mating rate/h of individual females before they were groomed for the first time by their new male with the mating rate/h after the first grooming incidence, using Wilcoxon signed 
rank test. Only the 15 females that were groomed by the new male at least once could be included in this analysis.

Finally, we tested the effect of the different mating tactics on female reproductive success. Binary logistic regression models were used to test whether the mating tactics affected a female's chances of becoming pregnant. The behaviour rates belonging to a female or male mating tactic were each added as predictor variable to a separate model, together with group ID as a covariate, and female pregnancy as the dependent variable. Female dominance rank was entered as a categorical predictor variable to be able to combine data from the four groups. Furthermore, we used Spearman rank correlation tests to study the effect of the mating tactics on the timing of giving birth.

$R$ version 3.6.0 with the package gmodels was used for analysis. All $p$-values were adjusted using Bonferroni correction, significance levels were set to $p \leq 0.05$.

\section{Results}

\section{Mating and births}

28 of the 43 studied females mated with the new male in their group. On average, the males mated $1.2 \pm 0.3$ times per hour with each female (Table 1 ). Mating rates did not significantly differ between the introductions (Kruskal-Wallis test: $\chi^{2}=3.701, \mathrm{df}=3$, $p=0.296)$.

\section{Female-new male approaches}

During the introductions, 25 of the 30 females approached their new male at least once during introductions A, C, and D, while the males approached 29 of the 30 females (Table 1). Note that there are no approach data from introduction B. The females approached their new male siginificantly more often than the male approached them (Wilcoxon signed-rank test: $N=43, Z=-3.201, p<0.001$ ). Additionally, females that approached their new male more often, also presented more often towards him (Spearman rank correlation test: $\mathrm{N}=30, \mathrm{R}_{\mathrm{s}}=0.610, \mathrm{p}<0.001$ ) and mated more often (Spearman rank correlation test: $N=30, R_{s}=0.809, p<0.001$ ), indicating that these particular females were sexually interested in their new male.

\section{Female-new male grooming}

Females may groom their new male in exchange for mating. Indeed, females that groomed their new male at least once were more likely to mate with him than females that never groomed their new male (Binary logistic regression, $N=43, z=3.412, p<0.001$ ). There were no differences between the introductions ( $p=0.634)$. To indentify whether females that groom longer also mate at higher rates, a model including female-new male grooming and group ID as predictor variables was run. The model showed that females that groomed their male longer mated more often (Generalized linear model, $\mathrm{N}=25, \mathrm{~F}(4,20)=6.804, \mathrm{p}=0.001$, Figure 1 ). This effect was significant in introduction $\mathrm{B}$ $(t=3.067, p=0.006)$, introduction $C(t=4.818, p<0.001)$, and introduction $D(t=2.128$, $p=0.046)$, but not in intoduction $A(t=0.960, p=0.249)$. This shows that there is an 
Table 1 The number of females, compared to the total number of females present, that engaged in interactions with the male, and the average occurrence (rate or \% of time) of each behaviour per introduction. FM = female to male, $M F=$ male to female, $F F=$ female to female

\begin{tabular}{|c|c|c|c|c|c|}
\hline Introduction & A & B & C & D & All \\
\hline Mating (n, rate/h) & $\begin{array}{c}11 / 16 \\
0.5 \pm 0.2\end{array}$ & $\begin{array}{c}7 / 13 \\
1.1 \pm 0.4\end{array}$ & $\begin{array}{c}5 / 9 \\
2.4 \pm 1.3\end{array}$ & $\begin{array}{c}5 / 5 \\
1.5 \pm 0.4\end{array}$ & $\begin{array}{c}28 / 43 \\
1.2 \pm 0.3\end{array}$ \\
\hline FM approaches (n, rate/h) & $\begin{array}{c}15 / 16 \\
0.8 \pm 0.2\end{array}$ & $x$ & $\begin{array}{c}5 / 9 \\
3.7 \pm 1.7\end{array}$ & $\begin{array}{c}5 / 5 \\
1.9 \pm 0.7\end{array}$ & $\begin{array}{c}25 / 30 \\
1.8 \pm 0.6\end{array}$ \\
\hline MF approaches (n, rate/h) & $\begin{array}{c}15 / 16 \\
0.26 \pm 0.1\end{array}$ & $x$ & $\begin{array}{c}9 / 9 \\
1.0 \pm 0.3\end{array}$ & $\begin{array}{c}5 / 5 \\
0.3 \pm 0.1\end{array}$ & $\begin{array}{c}29 / 30 \\
0.5 \pm 0.03\end{array}$ \\
\hline FM presenting ( $n$, rate/h) & $\begin{array}{c}11 / 16 \\
0.2 \pm 0.1\end{array}$ & $\begin{array}{c}10 / 13 \\
0.1 \pm 0.04\end{array}$ & $\begin{array}{c}9 / 9 \\
0.5 \pm 0.2\end{array}$ & $\begin{array}{c}5 / 5 \\
0.2 \pm 0.1\end{array}$ & $\begin{array}{c}35 / 43 \\
0.2 \pm 0.05\end{array}$ \\
\hline FF competition started ( $n$, rate/h) & $\begin{array}{c}11 / 13 \\
0.7 \pm 0.4\end{array}$ & $\begin{array}{c}10 / 11 \\
1.6 \pm 0.7\end{array}$ & $\begin{array}{c}8 / 9 \\
0.5 \pm 0.6\end{array}$ & $\begin{array}{c}5 / 5 \\
0.7 \pm 0.4\end{array}$ & $\begin{array}{c}34 / 38 \\
0.9 \pm 0.3\end{array}$ \\
\hline FF competition received ( $n$, rate/h) & $\begin{array}{c}13 / 13 \\
0.7 \pm 0.3\end{array}$ & $\begin{array}{c}11 / 11 \\
1.0 \pm 0.3\end{array}$ & $\begin{array}{c}8 / 9 \\
0.2 \pm 0.2\end{array}$ & $\begin{array}{c}4 / 5 \\
0.3 \pm 0.2\end{array}$ & $\begin{array}{c}36 / 38 \\
0.6 \pm 0.2\end{array}$ \\
\hline FM grooming ( $n, \%$ time) & $\begin{array}{c}9 / 16 \\
1.6 \pm 0.9\end{array}$ & $\begin{array}{c}6 / 13 \\
1.0 \pm 0.4\end{array}$ & $\begin{array}{c}5 / 9 \\
1.7 \pm 0.9\end{array}$ & $\begin{array}{c}5 / 5 \\
5.2 \pm 1.9\end{array}$ & $\begin{array}{c}25 / 43 \\
1.9 \pm 0.5\end{array}$ \\
\hline $\begin{array}{l}\text { FM grooming followed by mating } \\
\text { (\% grooming bouts) }\end{array}$ & 17.0 & 11.6 & 19.1 & 8.9 & 14.1 \\
\hline $\begin{array}{l}\text { Mating preceded by FM grooming } \\
\text { (\% mating) }\end{array}$ & 5.4 & 29.6 & 0.9 & 8.0 & 11.0 \\
\hline Forced mating ( $\mathrm{n} /$ total mating) & $1 / 222$ & 0/703 & $5 / 731$ & $1 / 703$ & $7 / 2358$ \\
\hline MF conflicts ( $n$, rate/h) & $\begin{array}{c}10 / 16 \\
0.3 \pm 0.2\end{array}$ & $\begin{array}{c}12 / 13 \\
0.1 \pm 0.02\end{array}$ & $\begin{array}{c}9 / 9 \\
0.3 \pm 0.1\end{array}$ & $\begin{array}{c}4 / 5 \\
0.2 \pm 0.1\end{array}$ & $\begin{array}{c}35 / 43 \\
0.2 \pm 0.1\end{array}$ \\
\hline MF grooming ( $n, \%$ time) & $\begin{array}{c}05 / 16 \\
0.05 \pm 0.03\end{array}$ & $\begin{array}{c}2 / 13 \\
0.02 \pm 0.01\end{array}$ & $\begin{array}{c}4 / 9 \\
0.09 \pm 0.06\end{array}$ & $\begin{array}{c}4 / 5 \\
0.22 \pm 0.07\end{array}$ & $\begin{array}{c}15 / 43 \\
0.7 \pm 0.02\end{array}$ \\
\hline $\begin{array}{l}\text { Change in mating after 1st MF } \\
\text { grooming (rate/h) }\end{array}$ & $-0.4 \pm 0.3$ & $-4.0 \pm 1.4$ & $-10.7 \pm 7.9$ & $-7.9 \pm 6.4$ & $-5.6 \pm 2.7$ \\
\hline
\end{tabular}

exchange between female-new male grooming and mating during part of the introductions. In particular, females groomed their male significantly more often on days they were sexually interested, compared to days they were not interested (Wilcoxon, $\mathrm{N}=21, \mathrm{Z}=-3.387, \mathrm{p}<0.001$, Figure 2 ), implying a direct link between grooming and mating. Overall, this implies that females may groom their male to gain mating access. 


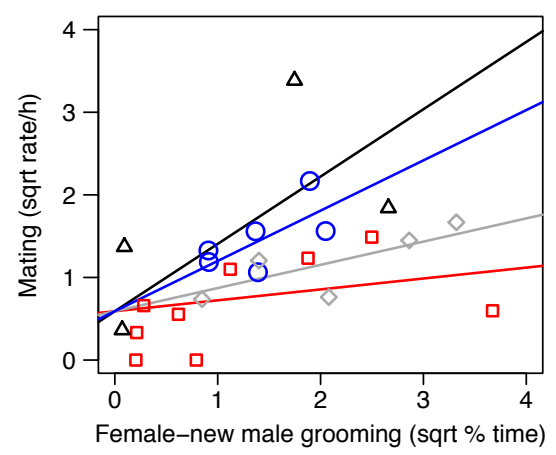

Figure 1 Female-new male grooming is related to increased mating access. Introduction $A=$ red squares, introduction $B=$ blue circles, introduction $\mathrm{C}=$ black triangles, introduction $\mathrm{D}=$ grey diamonds

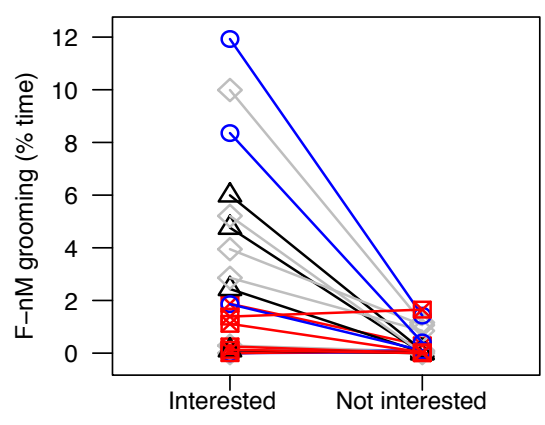

Figure 2 Female-new male grooming on the days females were sexually interested in the new male, compared to days they were not sexually interested. Introduction $\mathrm{A}=$ red squares, introduction $B=$ blue circles, introduction $C=$ black triangles, introduction $\mathrm{D}=$ grey diamonds

\section{Female dominance}

Female dominance rank did not affect the timing of the first mating between a female and their new male (Spearman rank correlation $A: N=16, R_{s}=0.388, p=0.548 ; B$ : $N=13, R_{s}=0.110, p=1.00 ; C: N=9, R_{s}=0.691, p=0.156 ; D: N=5, R_{s}=-0.154, p=1.000$ ), or how often she mated with the new male (Spearman rank correlation $A: N=16, R_{s}=-0.579$, $p=0.075 ; B: N=13, R_{s}=-0.275, p=1.00 ; C: N=9, R_{s}=-0.766, p=0.064 ; D: N=5, R_{s}=-0.400$, $p=1.000$ ). This indicates that females do not use dominance to secure mating access.

\section{Female-female aggression}

Females may aggress other females to secure mating access to their new male. Indeed, the number of female-female conflicts a female started (Friedman, $N=37$, $\chi^{2}=9.166, p=0.010$ ) and received (Friedman, $N=37, \chi^{2}=12.352, p=0.002$ ) was significantly affected by her sexual interest in the male. When sexually interested, females started and received aggression more often compared to the baseline period before the introduction (post-hoc Wilcoxon, started: $p=0.006$, received: $p=0.002$ ), and compared to days they were not sexually interested in their new male (post-hoc Wilcoxon, started: $p=0.001$, received: $p=0.007$, Figure $3-4$ ). Female-female aggression rates did not differ between the baseline and the days females were not sexually interested (post-hoc Wilcoxon, started: $p=1.000$, received: $p=0.151$ ). This shows that females especially aggress other females when they are interested in mating with the new male, and specifically target other sexually interested females.

Next, we studied whether female-female aggression over mating partners leads to increased mating access. Therefore, the change in female-female aggression was determined by subtracting the baseline aggression rate of female-female aggression from the female-female aggression rates on days they were sexually interested in their 


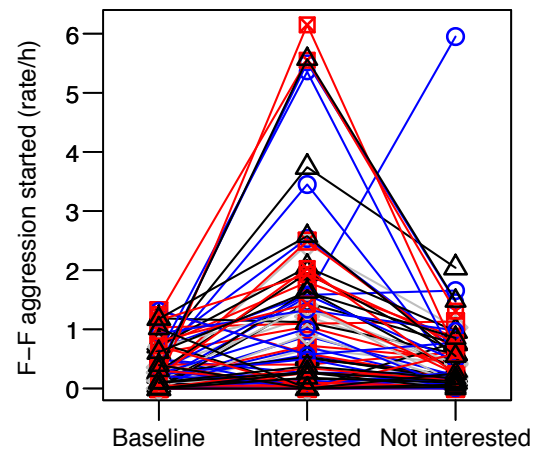

Figure $\mathbf{3}$ The rates at which individual females started female-female aggression during the baseline period, and during the introductions on days they were sexually interested, or not sexually interested in their new male. Each line represents a different female. Introduction $A=$ red squares, introduction $B=$ blue circles, introduction $\mathrm{C}=$ black triangles, introduction $\mathrm{D}=$ grey diamonds

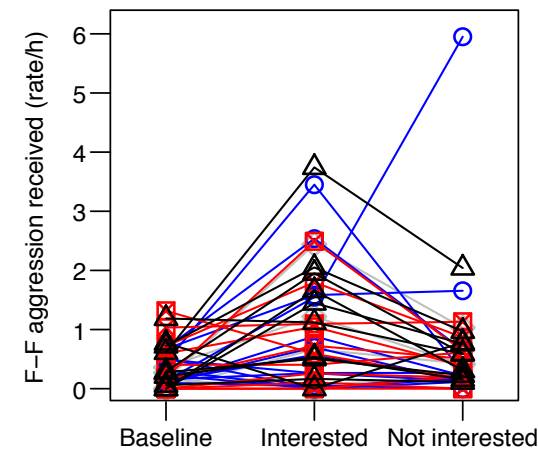

Figure 4 The rates at which individual females received female-female aggression during the baseline period, and during the introductions on days they were sexually interested, or not sexually interested in their new male. Each line represents a different female. Introduction $A=$ red squares, introduction $B=$ blue circles, introduction $\mathrm{C}=$ black triangles, introduction $\mathrm{D}=$ grey diamonds

new male. Females that had a higher increase in female-female aggression given had significantly higher mating rates with their new male (Spearman rank correlation, $N=37$, $\left.R_{s}=0.636, p<0.001\right)$. Yet, the change in receiving female-female aggression did not affect female mating access (Spearman rank correlation, $N=37, R_{s}=0.340, p=0.079$ ). Thus, females can increase their own mating access by engaging in female-female competition, but are not able to reduce the mating access of the females they compete with.

\section{Male mating tactics}

Not only the females may employ mating tactics to gain mating access to their new male, also the new males may use mating tactics. Males may use their dominance to gain mating access, use force or aggression for coercive mating, or groom females to gain mating access. First, all males obtained the alpha position in their new group, as they received submission from all females in their group at least once during the introduction. However, they mated with $54-100 \%$ of their females. This implies that obtaining the alpha position not necessarily grants a male mating access to all females.

Second, forced mating was rare: only 7 of the 2358 observed mating interactions were forced upon by the new males (Table 1). Moreover, there were no females that experienced forced mating only, and the first mating between a female and the new male was never forced. Thus, there is no evidence that the new males gained mating access using coercion. 
Third, females that mated with their new male more often, also received more male aggression (Spearman rank correlation test: $R_{s}=0.427, p=0.004$ ), the males may therefore use aggression as a mating tactic. However, females that were more often the target of male conflicts, also approached their male more often (Spearman rank correlation test: $R_{s}=0.446, p=0.014$ ) and presented more towards him (Spearman rank correlation test: $R_{s}=0.314, p=0.040$ ). This implies that male conflicts coincide with female interest and did not result in fear for the new male. Thus, there is no evidence that the females mated with the new male out of fear.

Fourth, the males groomed 15 of the 43 females, and spent only $0.07 \pm 0.02 \%$ of their time grooming the females (Table 1). A binary logistic regression model was ran to test whether the males particularly groomed the females they mated with. The model indeed showed that the males were more likely to mate with females they groomed at least once during the introductions (binary logistic regression, $N=43$, $z=2.333, p=0.020)$, this effect did not differ between the introductions $(p=0.339)$. Yet, there was no effect of the time a male groomed a female on their mating rate (Generalized linear model, $\mathrm{N}=15, \mathrm{~F}(4,10)=3.440, \mathrm{p}=0.051$ ). Additionally, mating rates were significantly lower after the first time a male groomed a female, compared to before the first grooming incidence (Wilcoxon signed-rank test: $N=15, Z=-2.939$, $p=0.008$, Figure 5). Taken together, this shows that new male grooming was rare and did not increase mating access to the females. Overall, only male aggression was related to mating, but this was not part of a coercive mating tactic.

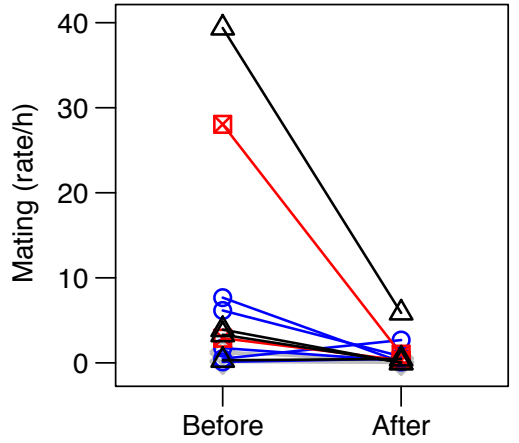

Figure 5 The significant decrease in mating rate/h when comparing mating before the first new male to female grooming incidence, with the mating rate/ $h$ after the first grooming incidence. Each line represents a different female. Introducion $A=$ red squares, introductio $B=$ blue circles, introduction $\mathrm{C}=$ black triangles, introduction $\mathrm{D}=$ grey diamonds

\section{Female reproductive success}

$65 \%$ of the females gave birth in the year after the introductions, varying from $33 \%$ to $77 \%$ of the females per introduction (Table 1). Females that mated with their new male sooner after the start of the introduction did not give birth sooner (Spearman rank correlation $\mathrm{N}=43, \mathrm{R}_{\mathrm{s}}=0.238, \mathrm{p}=0.124$ ), nor did the overall mating rate affect the timing of giving birth (Spearman rank correlation $N-43, R_{s}=0.238, p=0.124$ ). Still, the female and male mating tactics may affect female reproduction. 
Overall, the female-new male approach rate/h did not affect a female's chances of becoming pregnant (Binary logistic regression, $N=43, z=2.008, p=0.156$ ). Yet, there was a significant difference between the introductions $(p=0.013)$. Only during introduction $A$, females that approached their new male were more likely to become pregnant $(z=2.311, p=0.021)$. Additionally, the chances of female pregnancy were not affected by the time she groomed the new male (Binary logistic regression, $N=43, z=0.398, p=0.528$ ), her dominance rank (Binary logistic regression, $N=43, z=0.850, p=0.654$ ), or femalefemale competition (Binary logistic regression, starting: $N=37, z=2.592, p=0.107$; receiving: $N=37, z=2.312, p=0.128)$. There neither was an effect of female new-male approach rate/h (Spearman rank correlation, $N=43, R_{s}=-0.176, p=0.353$ ), female-new male grooming time (Spearman rank correlation, $N=43, R_{s}=0.129, p=1.000$ ), female dominance rank (Spearman rank correlation $A: N=16, R_{s}=-0.486, p=224 ; B: N=13$, $\left.R_{s}=-0.131, p=1.000 ; C: N=9, R_{s}=0.465, p=0.828 ; D: N=5, R_{s}=-0.564, p=1.000\right)$, or femalefemale competition on the timing of giving birth (Spearman rank correlation, started: $N=37, R_{s}=-0.078, p=1.000$; received: $\left.N=37, R_{s}=-0.308, p=0.126\right)$. Thus, none of the possible female mating tactics significantly affected female reproduction.

Finally, there was no overall effect of new male-female approach rate/h on the female's chances of becoming pregnant (Binary logistic regression, $N=43, z=0.448$, $p=0.503)$. Yet, also here the introductions differed significantly $(p=0.017)$. Females that received more approaches from their new male in introduction $A$ were more likely to become pregnant $(z=3.395, p=0.026)$. Additionally, there was no relation between new male-female grooming (Binary logistic regression, $N=43, z=0.312, p=0.571$ ) or new male-female aggression (Binary logistic regression, $N=43, z=1.220, p=0.270$ ) and a female's chance of becoming pregnant. Neither was the timing of giving birth dependent on new male-female approaches (Spearman rank correlation test, $N=43$, $R_{s}=-0.012, p=1.000$ ), new male-female grooming time (Spearman rank correlation test, $\mathrm{N}=43, \mathrm{R}_{\mathrm{s}}=-0.200, \mathrm{p}=0.594$ ), or new male-female conflicts (Spearman rank correlation test, $\left.N=43, R_{s}=-0.002, p=1.000\right)$. Thus, overall, none of the male mating tactics was linked to female reproductive success.

\section{Discussion}

This study aimed to identify whether female rhesus macaques used mating tactics to gain mating access to their new male during four male introductions, and linked these tactics to reproductive success. One-male groups were formed during the introductions. This resulted in a situation with limited and monopolisable mating access to a highly preferred male. If female primates are able to employ mating tactics, they are expected to do so in this highly competitive environment. Indeed, the females obtained mating access through approaching their new male and exchanged grooming for mating. Moreover, they aggressed other females to gain mating access to their new male. However, females employing mating tactics did not gain reproductive benefits. These results imply that females can play an active role in determining male mating access, and challenge the common believe that only males employ mating tactics in primates living in multi-male multi-female groups. 
Female-male approaches are often considered an expression of female mate choice interactions (Bercovitch 1991; Soltis et al. 1997; Setchell 2005; Arlet et al. 2007). Therefore, females interested in mating with their new male were expected to actively seek contact, and approach him often (Bercovitch 1991). Indeed, females commonly approached their new male, they even approached him more often than vice versa. This implies that the females were highly interested in social contact with their new male. As new males are considered preferred partners for females, it not surprising that several other studies report similar high female-new male approach rates (Henzi and Lucas 1980; Fragaszy et al. 1994; Olupot and Waser 2001). Additionally, females that approached their new male more often showed more sexual invitation behaviour and mated more often with their new male. Similar patterns are previously described in grey-checked mangabeys (Lophocebus albigena) and Japanese macaques (Soltis et al. 1997; Olupot and Waser 2001). This shows that social interest often coincides with a sexual interest. Taken together, our results imply that females maintain proximity to preferred males, which appears effective in obtaining mating access. Therefore, female-male approaches are an active expression of female mate choice and can be considered a female mating tactic.

In our highly competitive setting, we expected females to also employ other mating tactics than the active expression of female choice. Female-male grooming was expected to increase a female's mating access. Indeed, especially females that mated with their new male also groomed him. Moreover, the females in three of the four groups exchanged grooming for mating on the long term. These results confirm the relation between grooming and mating observed during male group entry in previous studies (Samuels and Altmann 1986; Cooper et al. 2001; Hayakawa and Soltis 2011). Moreover, they imply that females can use friendships with males as a mating tactic, similar to how primate males may benefit from friendships to gain mating access to females (Massen et al. 2012; Kulik et al. 2012; Ostner et al. 2013; Städele et al. 2019). Moreover, our results show that female-new male grooming often occurs on the same day as mating. However, grooming may not always precede mating. Females may especially groom males after copulating, to secure protection for themselves and their future offspring (Sonnweber et al. 2015). Indeed, female-new male grooming increases over time during introductions, while female mating with new males decreases over time (Rox et al. 2018). This implies that female-new male grooming occurs more often after (the first) mating. Thus, female-new male grooming may not necessarily serve to obtain mating access in the first place, but may function to maintain social and mating access on the long-term. Moreover, mating with a new male may promote the establishment of a social bond between females and the new male, which has been suggested previously (Sprague 1992). However, it remains unknown whether longlasting social bonds are formed during the mating season, as grooming between males and females within the mating season may not reflect what happens outside the mating season (Baxter and Fedigan 1979). Still, it is clear that female-new male grooming can be an effective female mating tactic during the mating season. 
Next, contrary to our predictions, female dominance did not affect female mating access. Thus, there is no evidence that dominant females can limit subordinates in their access to preferred mating partners. This is in line with research in yellow baboons, where female dominance rank was not related to sexual consort formation (Bercovitch 1991). Yet, our prediction was based on the fact that higher ranked females are more likely to have male friends than subordinates, indicating that dominant females can exclude low ranking individuals from access to valuable social partners (Palombit et al. 2001; Archie et al. 2014). Additionally, dominant females did not reproduce earlier than subordinates, which also contrasts with previous research on vervet monkeys (Chlorocebus pygerythrus) (Whitten 1983). What determines the presence or absence of link between access to males, female reproduction, and female dominance rank needs to be determined in the future. Possibly, social instability during male group entry plays a role, as all other studies are conducted in under socially stable circumstances (i.e. outside male group entry). During male introductions, female dominance rank does not affect when females engage in social interactions with a new male. Instead, female age plays an important role in providing social, and likely also sexual, access (Rox et al. 2018). Thereby, other female characteristics and female mating tactics may be more important than female dominance in determining who will interact with new males during introductions. Thus, the effect of female dominance on mating access may differ between social contexts.

Finally, we predicted that females would aggress other females to gain mating access. Indeed, females started more often conflicts with other females on the days they were sexually interested, and specifically directed these conflicts at other sexually interested females Moreover, females that engaged more in female-female aggression were more successful in obtaining mating access. This is in line with previous studies on chacma baboons, that describe that especially reproductively active females display high levels of female-female aggression, and direct this aggression at other reproductively active females (Huchard and Cowlishaw 2011; Baniel et al. 2018). Yet, these studies did not directly link female-female aggression to mating, but classified female-female conflicts as mating competition based on social and ecological factors. We are the first to directly link female-female conflicts to mating access. Thereby, our results indicate that femalefemale competition can be an effective female mating tactic.

The new males in our study did not use any mating tactics. This was predicted, as the new male was the limiting sex that potentially limited the females in their reproduction in our study. Generally, females are thought to be the limiting sex, forcing males to employ mating tactics to pursue reluctant females to mate. In our study, the male and female roles were reversed. Therefore it is not surprising that we did not find an effect of male dominance (Cowlishaw and Dunbar 1991; Berard 1999; RodriguezLlanes et al. 2009; Massen et al. 2012), male-female grooming (Massen et al. 2012; Ostner et al. 2013; Städele et al. 2019), or male coercion (Smuts and Smuts 1993; Clutton-Brock and Parker 1995; Soltis et al. 1997) on male mating access. Yet, the males mated more with females to whom they behaved more aggressively. Some researchers may argue this could be a form of males forcing females to mate, as 
females receiving more aggression may be too fearful to reject a male's mating attempt (Smuts and Smuts 1993). However, females that received more aggression from their new male also approached him more often and showed more sexual invitation behaviour. Thus, especially females that were interested in their new male received aggression, instead of male aggression leading to fear. Possibly, the increased aggression levels are linked to increased proximity, as was previously shown in other primate species (Carne et al. 2011; Crofoot et al. 2011). Females that spend more time with the male simply have more opportunity to receive aggression than females keeping their distance. On the long-term, the link between male aggression towards females and mating may disappear, as females receive less aggression from males they have close social bonds with (Haunhorst et al. 2017). In summary, there is no evidence for male coercion as a mating tactic in our study, neither did the males use any other mating tactics.

Female reproductive success was not affected by any of the studied behaviours. Females that employed mating tactics were not more likely to become pregnant, nor did they give birth earlier. This is in line with previous research stating that female mate choice does not affect paternity (Soltis et al. 2001; Engelhardt et al. 2006). Yet, these studies approached reproductive success from a male perspective, while we focus on female reproductive success. Moreover, the behaviours associated with female mating tactics are potentially costly, making it unlikely they do not result in any (reproductive) beneftis for females. Especially female-female aggression will demand more energy and comes with a risk of injury (Isbell 1991; Huchard and Cowlishaw 2011; MacCormick et al. 2012). Also female-new male grooming can be costly, as females can spend up to $13.5 \%$ of their time grooming a new male and lose valuable time. We expected females to gain reproductive benefits from displaying such costly behaviour, yet, the costs and benefits of female mating tactics are not reflected in our reproductive parameters. Likely, food abundance in captivity plays a role in the absence of a relation between female mating tactics and reproductive success. Captive females will be able to find high-quality food quickly. Thereby, they could easily compensate for the lost energy due to female-female competition. Additionally, spending more time grooming a male does not necessarily decrease their food intake, as there is no need for extensive foraging in captivity. Therefore, the effect of female mating tactics on reproductive success may differ between captive and wild groups. Additionally, the mating tactics may provide female with other benefits than reproductive benefits, such as increased bonding with the new male. Females can benefit from close social bonds with males, as males can provide services like protection for the female herself and her offspring (Engh et al. 2006; Palombit 2009; Sonnweber et al. 2015). Again, the effects of male protection may be more prevalent in the wild, where there are increased risks of predation and infanticide. Thus, it is hard to determine the costs and benefits of female mating tactics in captivity. Therefore, we cannot draw decisive conclusions on the fitness effects of female mating tactics in our captive study.

One important question that arises is how our results can be translated to the wild. We studied mating tactics in an extreme situation and approach our data from an 
experimental point of view. The circumstances during the male introductions are such that they are optimal for female competition over mates, and provide us the opportunity to study whether females are be able to use mating tactics. We showed that females indeed have the potential to employ mating tactics, a result that contrasts with many scientists' view on the battle of the sexes over mating (Hrdy 1999). Our findings in captive animals do not necessitate the occurrence of female mating tactics in natural situations. Still, it is unlikely female mating tactics evolved in the limited time the studied population spent in captivity. Therefore, also wild females may have power over with whom they mate. First, male group entry is relatively common in primates living in multi-male multi-female groups. Consequently, groups regularly contain a highly preferred male. Second, one-male macaque groups have been reported in the wild (Neville 1968; van Noordwijk and van Schaik 1985; Ménard and Vallet 1993; Keane et al. 1997), implying that also in the wild a male may sometimes be the limiting sex. Third, females can successfully reject male mating attempts (wild primates: (Bercovitch 1991, 1995; Arlet et al. 2007), captive primates: (Overduin-De Vries et al. 2012; Rox et al. 2018), showing they at least have some power over with whom they mate. Fourth, there are indications that also wild primate females may use mating tactics (wild macaques and baboons: Baniel et al., 2018; Huchard and Cowlishaw, 2011; Whitten, 1983). The next step is determining under which circumstances females use these mating tactics, and how they relate to the occurrence of male mating tactics. Females mating tactics are particularly expected in social contexts where there is limited access to preferred males. This is found when there is a large female-bias in sex-ratio, immediately after male group entry (i.e. when a highly preferred male is present) and when female synchrony in reproduction is high. Indeed, there is some evidence that females especially compete over access to males after male group entry (Baniel et al. 2018) and that the females' ability to overrule dominant males increased with higher reproductive synchrony (Ihara et al. 2016). Possibly, the occurrence of female mating tactics is negatively related to a male's coercive power. Males may be more able to force females to mate when sexual dimorphism is higher. Finally, is it important to realize that males and females likely both use mating tactics simultaneously. Females may employ mating tactics to gain access to their preferred male, while non-preferred males may attempt to pursue females to mate with them. Still, what social and environmental circumstance determine which female and male mating tactics occur needs to be studied in the future. Altogether, it is clear that both sexes have the potential to use mating tactics. Both males and females may choose to use mating tactics, or not use them at all.

\section{Conclusion}

To conclude, female primates have the potential to use mating tactics in a highly competitive situation. They actively seek mating opportunities with a male, and can actively compete with other females over mating access, and thereby increase their mating access to a new male. Yet, they are not able to affect other female's mating access. Moreover, females may groom males in exchange for mating. In contrast, the 
new males in our study did not use any mating tactics. These results show that females can play an active role in reproduction, which extends further than female mate choice and offspring rearing. They challenge the common believe that only males compete over mating partners and have the power to determine with whom they mate. However, the fitness consequences of female mating tactics remain unclear. Our research provides exciting opportunities for future research on the circumstances under which wild primate females and males use mating tactics. Likely, mating tactics can be flexibly used by both sexes, depending on the social context. 



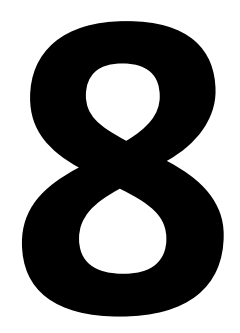

\section{A preliminary analysis of species differences in hair cortisol levels during male introductions in female macaques: a link to infanticide}

Astrid Rox, Linda Hofman, Lisette Minderman, Jan AM Langermans, Elisabeth HM Sterck 


\begin{abstract}
Male dispersal is necessary to prevent inbreeding. In primates, male group entry by dispersing males is associated with increased infanticide risk, and may cause stress in resident females. However, infanticide is not equally common in all primates: the risk is especially high in a-seasonally breeding species. In seasonally breeding species, the female stress response may depend on other factors, such as social buffering and baseline stress levels. This study aims to identify the long-term female stress response to new males, in species that differ in infanticide risk. Hair cortisol levels were measured in female long-tailed macaques (high infanticide risk) and rhesus macaques (low infanticide risk) before and after captive male introductions. During the introductions, hair cortisol levels increased in long-tailed macaque females, while there was no change in rhesus macaques. Overall, lactating females and females with a lower baseline cortisol level experienced more stress. Moreover, long-tailed macaque females that spent more time with the male, mated less with him, and had a more focused social network showed the largest increase in hair cortisol levels. In contrast, cortisol levels increased more in rhesus macaque females with a more dispersed social network. Altogether, the male introductions were most stressful for long-tailed macaque females; the species with a high infanticide risk. The introductions were particularly stressful for lactating females, who experienced highest direct infanticide risk. The stress response in female long-tailed macaques depended on social buffering and social behaviour linked to counterstrategies against infanticide, while there was an opposite effect of social buffering in rhesus macaques. Thus, there are species-specific stress responses of females to new males, which may be linked to infanticide risk.
\end{abstract}

\title{
Introduction
}

Male dispersal is common among primates, yet it causes stress in both residents (Alberts et al. 1992; Beehner et al. 2005; Bergman et al. 2005; Engh et al. 2006; Crockford et al. 2008; Cheney and Seyfarth 2009) and new males (Alberts et al. 1992; Bergman et al. 2005; Arlet et al. 2009). On the short-term, this stress response can help resident females to adequately respond to the risks associated with a new male entering the group (Charmandari et al. 2005; Cheney and Seyfarth 2009), such as high aggression levels (Lindburg 1969; Dittus 1975; Hrdy 1977; Packer and Pusey 1979; van Noordwijk and van Schaik 1985, 2000; Rox et al. 2018) and increased infanticide risk (Hrdy 1977, 1979; Palombit et al. 1997; van Schaik and Janson 2000; Fedigan 2003; Beehner et al. 2005; Engh et al. 2006; Lukas and Huchard 2014; Amann et al. 2017). However, females that receive more aggression from new males do not differ in their short-term stress response from females that receive little aggression (Alberts et al. 1992; Beehner et al. 2005). In contrast, especially pregnant and lactating females (i.e. the individuals that are at direct risk of infanticide) experience high short-term stress levels during male group entry, indicated by increased cortisol levels in faeces (Beehner et al. 2005; Engh et al. 2006). Moreover, observing actual infanticide may increase 
short-term stress levels in lactating females even further (Engh et al. 2006). Thus, not the rate of received aggression, but the infanticide risk is probably an important mediator of a female's stress response to male group entry.

For females at risk of infanticide, short-term stress is considered beneficial, since it elicits the correct responses (e.g. fight or flight) to threatening situations (i.e. offspring being attacked) and may thereby increase an individual's fitness. However, when this response is maintained for a longer period of time and results in long-term stress, indicated by long-term elevated levels of cortisol, animals may experience negative fitness consequences (Charmandari et al. 2005; Honess and Marin 2006; Cheney and Seyfarth 2009). Up to now, all studies on the female stress response during male group entry focused on the short-term stress response. The long-term consequences of an increased stress response due to high infanticide risk, and thereby its effect on female fitness, remain unknown.

It is known that infanticide is a great cost for primate females, who invest a large amount of time and energy in their offspring (van Schaik and Janson 2000). To lower the risks of infanticide, females developed several counterstrategies against infanticide. Lactating females may avoid new males (Hrdy 1979; Ebensperger 1998; Beehner et al. 2005), thereby preventing them to come near her dependent infant. In contrast, pregnant females may approach and mate with the new male in an attempt to confuse paternity (Hrdy 1977, 1979; Ebensperger 1998). Finally, pregnant females may terminate their pregnancies when a new male enters a group to prevent investing energy in offspring susceptible to infanticide, the so-called Bruce effect (Bruce 1960; Hrdy 1979; Ebensperger 1998; Roberts et al. 2012; Amann et al. 2017). Altogether, this illustrates that infanticide risk is an important factor determining a female's response to new males. Pregnant and lactating females that employ counterstrategies during male group entry may experience less stress than females that do not use any counterstrategies, as their infanticide risk is lowered.

Infanticide is, however, not equally common in all primate species. It is far more prevalent in a-seasonally breeding species, compared to seasonally breeding species (van Schaik and Janson 2000). In a-seasonal breeders, infants are born throughout the year and can be of any age when a new male enters the group, which also occurs throughout the year (e.g., long-tailed macaques (Macaca fascicularis): van Noordwijk and van Schaik 1985, 2000; reported infanticide: de Ruiter et al. 1994; Timmermans et al. 1981). Killing young infants could decrease the mothers' inter-birth interval, increasing a new male's reproductive opportunities (Hrdy 1977, 1979). In seasonal breeders, infants are born several months after the start of the breeding season, while male group entry is typically restricted to the months right before or at the start of the breeding season (e.g. rhesus macaques (Macaca mulatta): Lindburg 1969; Bernstein et al. 1977). During male group entry, infants are generally old enough to no longer inhibit their mother's fertility. As mothers are able to reproduce again during the breeding season, infanticide would typically not increase a male's reproductive opportunities. 
Infanticide risk is therefore expected to only influence the stress response to male group entry in a-seasonally breeding primates, and not in seasonally breeding primates. Other factors known to influence the stress response may be more prominent during male group entry in seasonal breeders.

Receiving social support may alleviate stress (Honess and Marin 2006; Brent et al. 2011). The presence of close kin can give social support to individuals through the formation of close bonds and social support in conflicts. Some studies indeed indicate that the presence of kin can buffer stress (Abbott et al. 2003), while others find no proof for such a relation (Crockford et al. 2008; Setchell et al. 2008). Primates not only form close bonds with kin, social bonds between non-related individuals are also common, so called friendships (Silk et al. 2003, 2009; Massen et al. 2010). However, not the number of friends, but rather the strength of social bonds appears important for buffering stress. Cycling females with a more focused social network, a measure for the strength of social bonds, showed a relatively small stress responses to a social stressor (Crockford et al. 2008; Wittig et al. 2008). Similarly, the presence of a specific male friend is an important buffer in pregnant and lactating females (Beehner et al. 2005; Engh et al. 2006). Male friends may serve as effective protectors against infanticide (Hrdy 1979; Palombit et al. 1997; Palombit 2009). Lactating female chacma baboons (Papio ursinus) with a male friend also experienced less stress after observing male infanticide, than lactating females without a male friend (Beehner et al. 2005; Engh et al. 2006). Overall, the strength of an individual's social bonds may determine its ability to socially buffer stress, regardless of whether their social network exists of related or unrelated individuals.

Finally, baseline stress levels may influence an individual's stress response. Animals with chronic higher baseline stress levels are generally less responsive to stressors than animals with lower baseline stress levels (Cheney and Seyfarth 2009). Therefore, females with lower baseline stress levels are expected to show a larger increase in stress during male group entry than females with high baseline stress levels. However, it remains unknown how the presence of kin, an individual's social network, and baseline stress levels influence the female stress response to male group entry in species that differ in their infanticide risk.

This study aims to identify the factors influencing the female stress response to male group entry in species with low and high infanticide risk. Therefore, we studied the long-term female stress response to male introductions in captive long-tailed macaques (a-seasonal breeders, high infanticide risk) and rhesus macaques (seasonal breeders, low infanticide risk), by comparing hair cortisol levels before and after the introductions. Moreover, we study the effect of individual characteristics, social behaviour and social buffering on the female stress response. Hair cortisol is a reliable indicator for long-term stress (Davenport et al. 2006). Due to the higher infanticide risk in long-tailed macaques, we expect the introductions to be stressful for long-tailed macaque females, but not for rhesus macaque females. All long-tailed macaque females are expected to show an increased stress response, irrespective of actual reproductive state. Long-tailed macaques are a-seasonal breeders and mate 
promiscuously, all females are therefore potentially at the early stage of pregnancy at the start of the introduction and may perceive infanticide risk. Pregnant and lactating females experience highest direct infanticide risk in both species, as infanticide is uncommon but not unseen in rhesus macaques (Camperio Ciani, 1984; BPRC records). Therefore, when considering individual characteristics, we expect pregnant and lactating females to experience more stress during the introductions. Additionally, females with high baseline cortisol levels are likely less responsive to stressors. Therefore, we expect females with a lower baseline cortisol level to experience more stress during the introductions. Displaying counterstrategies is expected to decrease the female's perceived infanticide risk, and thereby lower their long-term stress levels. Such strategies may only have evolved in species where infanticide risk is high. Therefore, female long-tailed macaques that spend less time with the new male and mate more with him are expected to have the lowest increase in hair cortisol levels. In contrast, we expect female rhesus macaques with more kin and a more focused social network to have the smallest increase in hair cortisol levels, due to (social) buffering of stress. Male aggression is not expected to influence the stress response in either of the species. Finally, we compared male and female social behaviour between the introductions, to ensure the observed effects are not due to differences in social behaviour.

\section{Methods}

\section{Subjects and housing}

Subjects of this study were twelve adult female long-tailed macaques (age 4-22), and 23 adult female rhesus macaques (age 3-20). The animals were housed in five different social groups at the Biomedical Primate Research Centre (BPRC) in Rijswijk, the Netherlands: two groups of long-tailed macaques (Haas and Lava group) and three groups of rhesus macaques (Front, Zwart and Xalie group). The groups had been established more than 10 years prior to this research, yet, the Lava group and Zwart group were the result of group fissions in the year before the introductions. These group fissions preserved the natural matrilines in the group, which are important for group-stability (McCowan et al. 2018; Rox et al. 2019). The groups consisted of the adult females (4-12 individuals) and their non-adult offspring (age <3 years, 3-11 individuals; Table 1). The groups were housed in spacious and enriched inside $\left(72 \mathrm{~m}^{2}\right.$, $2.85 \mathrm{~m}$ high) and outside ( $208 \mathrm{~m}^{2}, 3.1 \mathrm{~m}$ high) enclosures during the study (Vernes and Louwerse 2010).

Natural migration patterns were mimicked in the groups. Males were removed when reaching sexual maturity, while females remained in their natal group throughout their lives and form matrilines of related females. The groups consisted of two to four different matrilines (Table 1). Adult breeding males are replaced every four to five years to avoid inbreeding. As part of this replacement process, an unfamiliar adult male was introduced into each of the subject groups between 2010 and 2017. The males varied in age from $7-15$ years. Note that the rhesus macaque introductions 
took place during the breeding season. The males were gradually introduced into their new groups, following BPRC husbandry guidelines. All males were successfully introduced to their respective new group (see Rox et al. 2019).

At the start of the introductions, two long-tailed macaque females and six rhesus macaque females were pregnant. Animals were considered pregnant when paternity data confirmed that the infant born was fathered by one of the natal males removed from the group before the introduction started, or when a veterinarian indicated after physical examination that a female was pregnant at the time the introduction started. One pregnant long-tailed macaque female (Kaloa) had an abortion; she was observed carrying a premature baby during the introduction. A veterinarian indicated that the foetus was approximately 4 months old. Moreover, three of the long-tailed macaque females, including one of the pregnant females, and five of the rhesus macaque females were lactating at the start of the introduction. Animals were considered lactating when they had given birth to an infant less than 9 months before the start of the introduction. These infants were all observed suckling during the observation period. Females that were categorized as neither pregnant nor lactating were considered cycling. For the remainder of this paper, we will refer to pregnant and lactating females as non-cycling.

\section{Cortisol analysis}

Hair samples for cortisol analysis were collected during the yearly health checks of the monkeys. The animals are sedated during the health checks, and several health- and physiological measures are taken, including shaving some hair in the animal's neck. Hairs were collected from the skin up to the tip of the hair. The health checks took place between 297 and 249 days before the introductions started, and between 33 and 109 days after the start of the introductions. Hair samples collected before the introductions were considered baseline samples. Samples of the Zwart group before the introduction were not available, therefore, hair samples collected 428 days (i.e. the year after) after the introduction were used as baseline samples for this group (Table 1).

It is crucial that the cortisol levels in the hairs collected right after the introductions reflect the introduction period. Often, an area may be shaven before the stressful event and be shaven again for sample collection, to ensure measuring post-event stress levels only (Davenport et al. 2006; Yamanashi et al. 2013). However, the method of sample collection in our study was limited by the non-invasive nature of our study. The hair samples we used were collected during the yearly health checks of the animals, without shaving prior to the male introduction. This method is also reported in previous studies (Kirschbaum et al. 2009; Fairbanks et al. 2011). Moreover, the time frame for collecting hair samples after the introductions in comparable to the time frame used by Davenport et al. 2006. Therefore, we expect that the cortisol levels in the hair samples represent the introduction period.

The hair samples were packed in tin foil right after collection and stored in a freezer under- $20^{\circ} \mathrm{C}$. The samples were retrieved in September 2017 (Haas and Front group) and March 2019 (Lava, Zwart, and Xalie group), after being stored in the freezer for 0.5-8 
Table 1 The composition of the study groups, and the details on the introductions and data collection during our study. LTM = long-tailed macaques, Rhesus = rhesus macaques, yr. = year

\begin{tabular}{|c|c|c|c|c|c|}
\hline Group & Haas & Lava & Front & Zwart & Xalie \\
\hline Species & LTM & LTM & Rhesus & Rhesus & Rhesus \\
\hline Adult females ( $n$ ) & 12 & 4 & 11 & 8 & 6 \\
\hline Sub-adult males ( $n$ ) & 0 & 1 & 0 & 2 & 0 \\
\hline Non-adults (n) & 11 & 3 & 3 & 9 & 6 \\
\hline Matrilines ( $\mathrm{n}$ ) & 4 & 2 & 2 & 2 & 4 \\
\hline Male age (yr.) & 15 & 8 & 13 & 10 & 7 \\
\hline Start introduction & $01 / 25 / 2017$ & 02/10/2015 & $12 / 19 / 2016$ & $12 / 23 / 2010$ & $12 / 01 / 2011$ \\
\hline End introduction & 03/06/2017 & 03/26/2015 & $12 / 30 / 2016$ & $12 / 29 / 2010$ & $02 / 27 / 2012$ \\
\hline Baseline samples & 04/19/2016 & 06/06/2014 & 03/08/2016 & $02 / 24 / 2012$ & $02 / 07 / 2011$ \\
\hline Introduction samples & 05/03/2017 & 04/01/2015 & 04/07/2017 & $01 / 25 / 2011$ & $02 / 16 / 2012$ \\
\hline Females included (N) & 10 & 2 & 11 & 7 & 5 \\
\hline Pregnant females (N) & 2 & 0 & 4 & 2 & 0 \\
\hline Lactating females (N) & 3 & 0 & 0 & 2 & 3 \\
\hline Behavioural data & Yes & No & Yes & No & No \\
\hline
\end{tabular}

years. The protocol of Davenport et al. 2006 was used to examine the cortisol levels in the hair samples. The complete (i.e. from the skin to the tip) hairs within each sample were mixed and washed with isopropanol twice for three minutes. After drying for 5 days on room temperature, $80-100 \mathrm{mg}$ of each hair sample containing complete hairs was ground into a fine powder using a bead beater. 50mg of powdered hair was collected from each sample and mixed with $1 \mathrm{ml}$ of methanol. The samples were left to incubate for 24 hours on a slowly rotating plate at room temperature, followed by centrifugation at a speed of $2500 \mathrm{rpm}$ for approximately 5 minutes. $0.6 \mathrm{ml}$ of the methanol mixture was transferred into a new tube, and left to dry on a $45^{\circ} \mathrm{C}$ heating block for approximately 5.5 hours. Then, $0.4 \mathrm{ml}$ phosphate buffer was used to liquefy the dried extract and the tubes were shortly vortexed. An enzyme immunoassay kit (Salimetrics: Expanded Range High Sensitivity Salivary Cortisol Enzyme Immunoassay Kit) was used to qualify the cortisol level in each sample. The steps in the instruction manual delivered with the kit were followed. The obtained optical density values were used to calculate the cortisol level in each sample, as indicated by the manual. Finally, the cortisol values were corrected for powder weight by calculating the pg cortisol/mg hair.

To control for the influence of other social stressors or management procedures on the hair cortisol levels, we only analysed samples from females that did not experience any potentially highly stressful events in the three months before the baseline sample 
was collected. Four long-tailed macaque females and two rhesus macaque females were excluded from analysis. These animals were caught and sedated for medical treatment ( $\mathrm{N}=2$ long-tailed macaques, $\mathrm{N}=1$ rhesus macaque), or experienced a female rank reversal ( $\mathrm{N}=2$ long-tailed macaques). Additionally, the hair samples of one rhesus macaque female were not coded reliably and could not be included in our analysis. Samples of 12 long-tailed macaques and 23 rhesus macaques remained for analysis (Table 1).

\section{Behavioural observations}

Behavioural observations were conducted during two of the introductions (longtailed macaques: Haas group, rhesus macaques: Front group). In total, behavioural data were collected on 10 long-tailed macaque and 11 rhesus macaque females (Table 1). Observations were carried out from two weeks before an introduction until the introduction started (i.e. pre-introduction phase) and from the first day of the introduction until the male was allowed to remain in the group full-time. During the pre-introduction phase, scan sampling of the whole group was done to identify the female social network in a group. Scan samples were taken every 30 minutes on 8 different days between $09.00 \mathrm{am}$ and $11.30 \mathrm{am}$, and between $01.00 \mathrm{pm}$ and $03.30 \mathrm{pm}$. In each scan we noted all individuals that were visible and which animals were in their proximity $(<1 \mathrm{~m})$. In total, 79 (long-tailed macaques) and 86 (rhesus macaques) scan samples were collected. During the introductions we conducted focal animal sampling on the new male while recording all interactions with his female interaction partners. All aggressive behaviour he displayed towards and received from the females, the time he spent in proximity with the females, and how often he mated with each female was noted. The subjects had access to both their inside and outside enclosure during all observations during the pre-introduction and part of the introduction (see detailed introduction procedure in Rox et al. 2018). The location of the observations (either inside or outside) was determined by a semi-random balanced schedule. We recorded how often each female was visible when the new male was focalled. Finally, we scored submissive behaviour (bared teeth and displacement) between all individuals in a group ad libitum during all observations.

\section{Measures}

The hair samples collected before the introductions were used to calculate the baseline cortisol level for each individual. The change in hair cortisol levels as a result of the introduction (named the change in cortisol in the remainder of this paper) was calculated by subtracting the baseline cortisol level from the cortisol levels observed after the introduction. A positive reading indicates an increase and a negative reading a decrease in hair cortisol level. The effect of different factors on the change in cortisol was tested.

First, individual characteristics of all 35 subjects were determined. This concerned species, age, pregnancy, lactation status, and the duration of the male introduction. We calculated the individual's age on the first day of the introductions. Pregnancy and 
lactation status were also determined on the first day of the introductions, as defined previously (see Subjects and Housing). The duration of the introductions was determined by counting the number of days between the day a male was physically introduced to the entire group, and the day the male was allowed to stay with the group full-time. Rhesus macaque introductions last 44 days on average (Rox et al. 2019). We considered all introductions lasting 44 day \pm 11 days $(=25 \%)$ of average duration. Introductions that lasted less than 33 days were considered short, while introductions taking more than 55 days were considered long. Transformation to a categorical variable was necessary to ensure the variable fit with the model assumptions.

Second, we focused on interactions between the females and the new male, and social buffering. Therefore, observational data collected during two introductions (the Haas group and Front group) were used to calculate the rate/h of male to female and female to male aggression, female to male submission, and mating, based on the time each female was visible to the observer. Moreover, we derived the percentage of time each female spent in proximity with the new male from these data. Next, the number of close matrilineal kin of each female (relatedness $=0.5$, i.e. mothers, siblings and offspring) was counted, based on the family tree of the groups. Additionally, the percentage of time a female spent in proximity with each individual in the group was calculated, based on the scan samples from the pre-introduction phase. From these proximity data, the Shannon-Wiener Diversity Index (SWDI) was calculated as a measure of the distribution of an individual's social network among all possible different social partners in a group (Crockford et al. 2008; Wittig et al. 2008). The following formula was used to calculate the SWDI:, with being the proportion of time that is spent in proximity of individual, indicates the total number of individuals with whom an animal spent time in proximity. A low SWDI indicates a skewed and focused social network, while a high SWDI indicates a more dispersed and equally distributed social network.

\section{Statistics}

First, we tested whether the hair cortisol levels were significantly higher after the introductions than before, using data from all 35 females. This was done for the two species separately, as there were more rhesus macaques in our sample than longtailed macaques. We expect differences between the species, and the effect of the rhesus macaques may overrule a different effect in our limited sample of long-tailed macaques. A paired samples t-test was used to test the difference between baseline and introduction cortisol levels in the long-tailed macaques, while Wilcoxon signed ranks test was used for the same analysis in rhesus macaques. Moreover, a MannWhitney $U$ test was used to compare the baseline cortisol levels between the species.

Second, we studied the effect of individual characteristics on the long-term stress response to male introductions. A linear regression model with the change in cortisol as dependent variable was ran with data from all 35 subjects. Species, the log of female age, the log of baseline cortisol levels, the duration of the introductions, pregnancy* 
species, and lactation*species were added as predictor variables. Log transformation was applied to female age and baseline cortisol levels to fit with the assumptions for linear regression model. Moreover, the interaction effects between pregnancy and species and between lactation and species were added because a species difference was expected between species with high and low infanticide risk. After composing the model, stepwise backward model selection based on AIC, from the MASS package in R, was used to select the best predicting model. Factors were considered significant contributors to the final model when $\Delta \mathrm{AIC} \geq 2$ compared to the null-model.

Third, we compared male-female and female-male behaviour between the two introductions during which behavioural observations were conducted. We used data from all individuals in the group; 12 long-tailed macaque females and 11 rhesus macaque females. Mann-Whitney $U$ tests were used to test for a difference in the rate of male-female aggression, the rate of female-male aggression, the percentage of time females spent in proximity with the new male, the mating rate between the female and the new male, and the female-male submission rates. The obtained $p$-values were adjusted for multiple testing using Bonferroni correction.

Fourth, tested whether male-female aggression, female-male behaviour, or social buffering affected the change in cortisol. This analysis was conducted for each species separately, as differences between the species were expected. Data from all females that were the subject of behavioural observations during the introductions and had reliable baseline cortisol samples were included in the analysis ( $N=10$ long-tailed macaques, $\mathrm{N}=12$ rhesus macaques). The change in cortisol was entered as dependent variable to a linear regression model. For the long-tailed macaques, female-male aggression rates, the log of the mating rate, the log of the time spent in proximity, the number of kin, and the SWDI were added as predictor variables. Log transformation was necessary on the mating rate and the time females spent in proximity to fit with the assumptions for linear regression models. For the rhesus macaques, the binary occurrence (yes/no) of male-female aggression, the occurrence of mating, the occurrence of female proximity with the male, the number of female kin, and the SWDI were added as predictor variables. It was necessary to transform the interaction rates between the females and the male to binary occurrence because there were too many zeroes in the data to comply with the assumptions for linear regression model. After composing the models for each species, stepwise backward model selection based on AIC was used to select the best predicting models. Factors were considered significant contributors to the final model when $\Delta \mathrm{AIC} \geq 2$ compared to the null-model.

Finally, we tested whether the factors that significantly predicted the change in cortisol in either of the analysis were linked. These analyses could only be conducted on the females that were included in all analysis ( $N=10$ long-tailed macaques, $N=12$ rhesus macaques). For the long-tailed macaques, we used independent samples t-test to test whether lactating females differed in their SWDI, the log of the time they spent in proximity, and the log of the mating rate. Moreover, Pearson correlation tests were used to correlate the baseline cortisol levels of long-tailed macaque females to their SWDI, the log of the time they spent in proximity, and the log of the mating rate, For 
the rhesus macaques, Spearman rank correlation test was used to correlate the log of the baseline cortisol level with the SWDI. The effect of lactation on SWDI could not be tested in the rhesus macaques, as there were no lactating females present during the introduction in the Front group.

All statistical analyses were done with R Studio version 1.1.4, with installed packages GGplot2, JTools, Effects, and MASS. Testing was two-sided and significance level was set to $p \leq 0.05$.

\section{Results}

\section{Increase in cortisol}

To study whether male introductions led to a change in cortisol, we compared cortisol levels before and after the introductions for each female. Hair cortisol levels increased significantly in the long-tailed macaques, from $35.65( \pm 2.84) \mathrm{pg} / \mathrm{mg}$ hair during the baseline to $46.49( \pm 4.98) \mathrm{pg} / \mathrm{mg}$ hair after the introductions (paired samples t-test, $N=12, t=2.527, p=0.028$; Figure 1 ). In contrast, there was no significant difference in the rhesus macaques (Wilcoxon signed ranks test, $Z=1.020, p=0.846$ ). Their baseline cortisol levels were $48.21( \pm 4.76) \mathrm{pg} / \mathrm{mg}$ hair, while the hair cortisol levels after the introduction were $48.04( \pm 4.12) \mathrm{pg} / \mathrm{mg}$ hair (Figure 1$)$. There was no significant difference in the baseline cortisol levels between the species (Mann-Whitney $U$ test, $\mathrm{N}=12 / 23, \mathrm{U}=178, \mathrm{p}=0.172$ ).
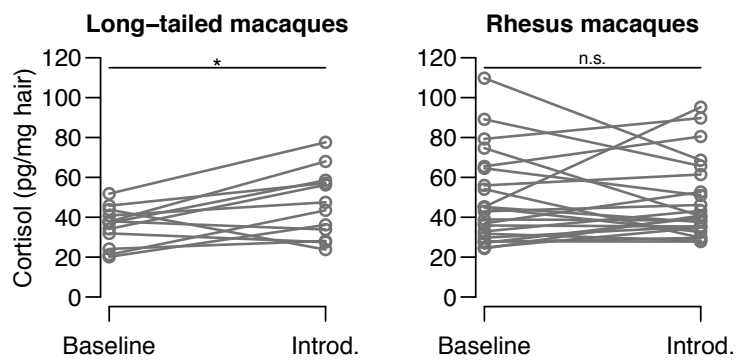

Figure 1 The change in hair cortisol levels from the baseline to after the introductions (introd.) in long-tailed macaque (left, $\mathrm{N}=12$ ) and rhesus macaque females (right, $\mathrm{N}=23$ ). Each line represents a different female.

\section{Individual characteristics}

We studied the effect of individual characteristics on the change in cortisol, using stepwise backwards regression models. A model including female age, pregnancy, the duration of the introduction, lactation, and baseline cortisol levels best predicted the change in cortisol (Linear regression model: $F(5,29)=5.937, R^{2}=0.505, p<0.001$ ). Yet, $\Delta$ AIC was lower than 2 for female age, pregnancy and the duration of the introduction (Table 2). Therefore, we only considered lactation and baseline cortisol levels significant predictors of the change in female hair cortisol levels in response to male introductions. 
In fact, lactating females experienced more stress than females that were not lactating, and females with higher baseline cortisol levels experienced less stress during the introductions (Figure 2).

Table 2 The steps and outcome of the stepwise backward regression model, with the change in cortisol as the dependent variable and different female characteristics as predictor variables.

\begin{tabular}{lc|lr|lc|lc}
\multicolumn{2}{c|}{ Step 1 } & & \multicolumn{2}{c|}{ Step 2 } & \multicolumn{2}{c}{ Step 3 } & \multicolumn{2}{c}{ Step 4 } \\
\hline Variable & $\Delta$ AIC & Variable & $\Delta$ AIC & Variable & $\Delta$ AIC & Variable & $\Delta$ AIC \\
\hline $\begin{array}{l}\text { Lactation* } \\
\text { Species }\end{array}$ & -1.82 & $\begin{array}{l}\text { Pregnancy* } \\
\text { Species }\end{array}$ & -1.23 & Species & -1.99 & - & 0 \\
Pregnancy* & -1.12 & - & 0 & - & 0 & Age & 1.16 \\
Species & 0 & Age & 0.87 & Age & 0.78 & Pregnancy & 1.21 \\
Age & 0.82 & Duration & 1.25 & Pregnancy & 1.11 & Duration & 1.73 \\
Duration & 1.36 & Lactation & 8.11 & Duration & 1.74 & Lactation & 8.14 \\
Baseline cortisol & 14.16 & Baseline cortisol & 15.17 & Lactation & 7.66 & Baseline cortisol & 18.53
\end{tabular}
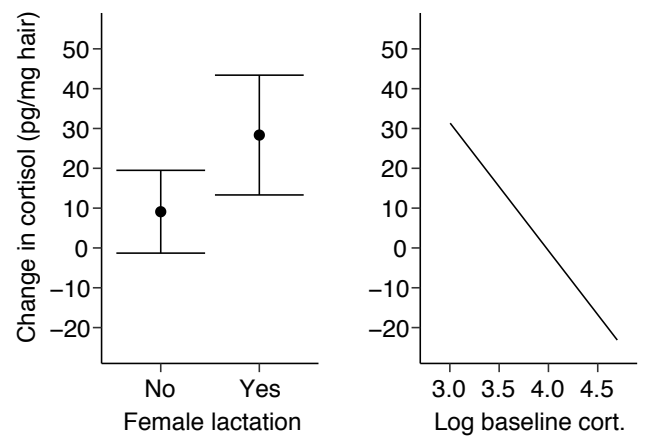

Figure 2 The individual characteristics significantly predicting the change in female hair cortisol in response to male introductions (mean $+95 \%$ confident interval). Lactating females and females with lower baseline cortisol levels experienced more stress during the introductions.

\section{Species differences in behaviour}

Next, we studied the effect of behaviour and social buffering on the change in cortisol. Only females from the two introductions where behavioural observations were conducted could be included in this analysis. We compared male-female aggression and female-male behaviour between the two species. Both male-female aggression (Mann-Whitney $\mathrm{U}, \mathrm{N}=12 / 11, \mathrm{U}=110, \mathrm{p}=0.10$ ) and female-male aggression (Mann-Whitney $U, N=12 / 11, U=126, p<0.001$ ) were higher during the long-tailed macaque introduction, compared to the rhesus macaque introduction. There were no significant differences between the species in the time females spent in proximity with the male (Mann-Whitney $U, N=12 / 11, U=93, p=0.545$ ), female mating with the new male (Mann-Whitney $U, N=12 / 11, U=85, p=0.705$ ), and female-new male submission rates (Mann-Whitney $\mathrm{U}, \mathrm{N}=12 / 11, \mathrm{U}=94, \mathrm{p}=0.180$ ). Thus, the long-tailed macaques were more aggressive than the rhesus macaques, while other behaviours did not differ between the species. 


\section{Effect of behaviour and social buffering}

For the long-tailed macaques, a model including mating, SWDI and proximity best predicted the change in cortisol during the introductions (Linear regression model: $F(3,6)=46.55, R^{2}=0.959, p<0.001$ ). $\Delta$ AIC was higher than 2 for each of the factors (Table $3)$. Therefore, we consider all three factors significant predictors of the change in cortisol in long-tailed macaques. In particular, females that mated less with the new male, had a more focused social network and spent more time in proximity with the new male had the highest increase in hair cortisol levels (Figure 3).

For the rhesus macaques a model including the occurrence of mating, the occurrence of male-female aggression, number of close kin and SWDI best predicted the chance in cortisol (Linear regression model: $F(4,6)=6.966, R^{2}=0.823, p=0.019$ ). Yet, $\triangle \mathrm{AIC}$ was lower than two when comparing the number of kin, male-female aggression, and mating to the null-model (Table 4). Therefore, SWDI is considered the only significant predictor of the change in cortisol in response to the male introduction in rhesus macaques. In fact, females with a more dispersed social network had the highest increase in hair cortisol levels (Figure 4).

Table 3 The steps and outcome of the stepwise backward regression model for the long-tailed macaques, with the change in cortisol as the dependent variable and behaviour and kin as predictor variables.

\begin{tabular}{lc|lc|lc}
\multicolumn{1}{c|}{ Step 1 } & & \multicolumn{2}{c|}{ Step 2 } & \multicolumn{2}{c}{ Step 3 } \\
\hline Variable & $\Delta$ AIC & Variable & $\Delta$ AIC & Variable & $\Delta$ AIC \\
\hline Male-female aggression & -1.99 & Kin & -1.83 & - & 0 \\
Kin & -1.96 & - & 0 & Mating & 7.07 \\
- & 0 & Mating & 7.22 & SWDI & 13.49 \\
Mating & 7.23 & SWDI & 13.42 & Proximity & 23.94 \\
SWDI & 8.43 & Proximity & 24.08 & & \\
Proximity & 20.05 & & & &
\end{tabular}
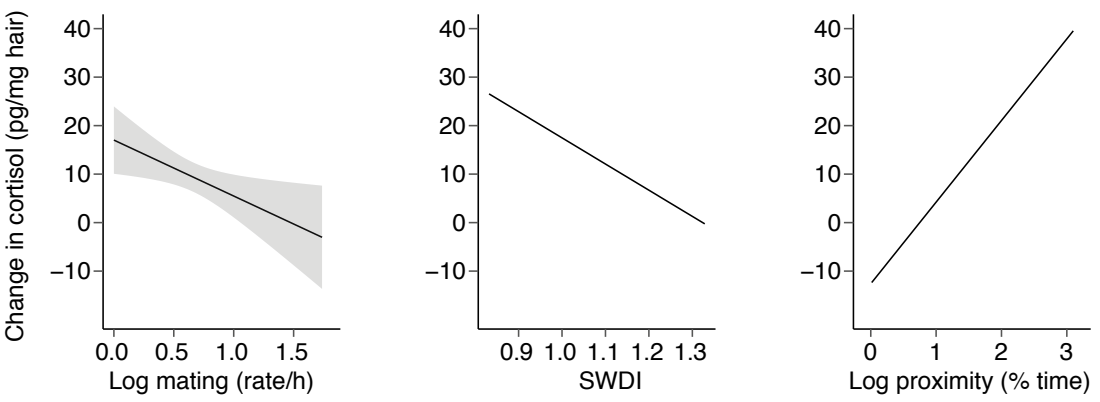

Figure 3 The behavioural factors significantly predicting the change in female hair cortisol in response to male introductions (mean + 95\% confident interval) in long-tailed macaques. Females that mated less, has a more focused social network and spent more time in proximity with the male experienced more stress. 
Table 4 The steps and outcome of the stepwise backward regression model for the rhesus macaques, with the change in cortisol as the dependent variable and behaviour and kin as predictor variables.

\begin{tabular}{lc|lc}
\multicolumn{2}{c|}{ Step 1 } & \multicolumn{2}{c}{ Step 2 } \\
\hline Variable & $\Delta$ AIC & Variable & $\Delta$ AIC \\
\hline Proximity & -1.54 & & 0 \\
Mating & -0.31 & Mating & 1.17 \\
- & 0 & Male-female aggression & 1.26 \\
Male-female aggression & 1.41 & Kin & 1.74 \\
Kin & 1.71 & SWDI & 12.00 \\
SWDI & 11.60 & &
\end{tabular}

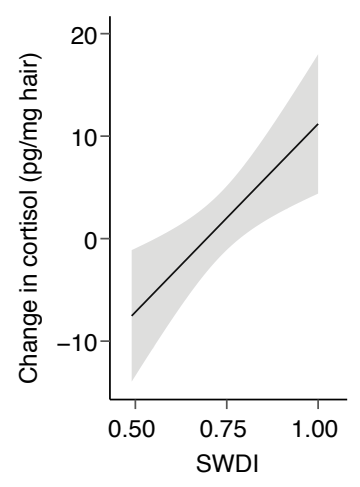

Figure 4 SWDI as the only significant predictor of the change in female hair cortisol in response to male introductions (mean + 95\% confident interval) in rhesus macaques. Females with a more dispersed social network experienced more stress.

\section{Link between significantly predicting factors}

Finally, we studied whether the behavioural parameters that significantly predict the change in cortisol, are linked to the individual characteristics that also affect the change in cortisol in our larger sample. Unfortunately, the sample in this preliminary study was too small to add all parameters to the same model. Still, this post-hoc analysis can provide valuable information for future analysis with a larger sample.

Lactating female long-tailed macaques did not differ from non-lactating females in their SWDI (independent samples t-test, $N=10, t=-0.601, p=0.567$ ), the time they spent in proximity with the male (independent samples $t$-test, $\mathrm{N}=10, \mathrm{t}=-1.899, \mathrm{p}=0.128$ ), or their mating rate (independent samples t-test, $\mathrm{N}=10, \mathrm{t}=-0.582, \mathrm{p}=0.590$ ). Additionally, baseline cortisol levels of the long-tailed macaque females were not correlated to the female's SWDI (Pearson correlation: $N=10, R=0.244, p=0.498$ ), the time females spent in proximity with the male (Pearson correlation: $N=10, R=0.162, p=0.655$ ), or their mating rate with the new male (Pearson correlation: $N=10, R=-0.006, p=1.000$ ). Thus, there was no relationship between any of the factors affecting the change in cortisol in the different analysis for the long-tailed macaques. This implies that all factors that affect the change in cortisol in response to male introductions act independently of each other. 
Rhesus macaque females with higher baseline cortisol levels had a lower SWDI (Spearman rank correlation, $\mathrm{N}=11, \mathrm{R}_{\mathrm{s}}=-0.897, \mathrm{p}<0.001$ ). Implying a possible link between the two analyses for this species. Because there were no behavioural data on lactating rhesus macaque females it was not possible to test for a link between lactation and SWDI in this species.

\section{Discussion}

This study focused on species-specific female stress responses to male introductions, based on differences in infanticide risk. The long-term stress response to male introductions, and the individual characteristics and behaviour influencing this response were compared between long-tailed macaque and rhesus macaque females. Long-tailed macaques are a-seasonal breeders and are more at risk of infanticide than the seasonally breeding rhesus macaques. The long-tailed macaque females showed a significant increase in cortisol levels during the introduction, while there was no change in the rhesus macaque females. Overall, the change in cortisol was highest in lactating females and females with lower baseline cortisol levels, without species differences. However, when analysing social behaviour and social buffering, we found behaviour linked to female counterstrategies to infanticide and the social network influenced the stress response in long-tailed macaque females, while the stress response in rhesus macaque females was only related to their social network. Overall, these results indicate differences in the stress response to male introductions in species that differ in their infanticide risk.

\section{Sample collection}

The non-invasive nature of the study determined the method of hair sample collection. We could not shave an area prior to the introduction to ensure measuring post-introduction cortisol levels only. However, our hair collection procedure cannot explain the observed difference between the two species in the increase in hair cortisol levels with male introductions. First, previous research has indicated that a stressful event can be traced back in hair cortisol levels for at least 14 weeks (Davenport et al. 2006). We used this time frame for post-introduction sample collection in both species, which implies that our measurements included cortisol deposited in the hair postintroduction. Even if cortisol from before the introduction was included in our hair samples, this would only concern a limited amount of time. Second, no management procedures that may cause stress (e.g. catching animals in the group for veterinary checks) occurred in either of the study groups in the months prior to sample collection, outside the described introductory procedure. Third, all groups were considered socially stable as no social unrest, increased aggression levels or increased injury rates (cf. Shively et al. 1986) were reported in the months prior to sample collection. Thus, it is unlikely that the increase in hair cortisol levels in female long-tailed macaques is linked to any other obvious stressful event other than the male introduction. Therefore, we consider the hair cortisol levels in our study a reliable indicator of the stress induced through male introductions. 


\section{Behavioural differences between the species}

Male and female social behaviour was compared between the species to explore whether the observed differences in the stress response can be linked to differences in behaviour. The species did not differ in proximity levels, mating rates and submission rates during the introductions. These results are in line with previous research, that reports similar patterns in female to new male behaviour between introductions (Rox et al. 2018). In contrast, the long-tailed macaque females showed higher aggression levels towards the new male compared to the rhesus macaque females, and also received higher levels of aggression. Females from species with high infanticide risk may be more hostile against new males, who pose a large risk for their offspring. Indeed, there is evidence that infanticide risk can mediate female behaviour towards new males (Beehner et al. 2005; Ichino 2005; Fruteau et al. 2010; van Belle et al. 2010; reviewed in Chapter 2). The males, in their turn, may respond to these aggressive females by behaving more aggressively himself.

Our results on the factors mediating the female stress response to male introductions were likely not affected by these differences in aggression between the species. Male aggression towards a specific female did not alter her stress response. This is in line with previous research in baboons (Alberts et al. 1992; Beehner et al. 2005). As male aggression does not influence the female stress response, while infanticide risk likely does (Beehner et al. 2005; Engh et al. 2006), this suggests that male aggression towards resident females is independent of infanticide.

\section{Infanticide risk}

Females of the two species differed in their stress response to the introduction of a new male. As expected, there was a significant increase in stress levels in response to male introduction in long-tailed macaque females, but not in rhesus macaques. These cortisol levels indicate that long-tailed macaque females thus experienced the male introduction as more stressful than the rhesus macaque females. This fits with the notion that the risk of infanticide enhances the female stress response to male group entry (Beehner et al. 2005; Engh et al. 2006). Yet, we have only limited data on the female stress response during male introductions in long-tailed macaques $(N=12)$. Data from more long-tailed macaque introductions are needed to see whether this pattern is consistent across long-tailed macaque groups.

Another factor that could cause such differentiated stress responses between the species is a difference in baseline cortisol levels. Females with higher baseline cortisol levels experiences showed smaller increases in cortisol during the introductions, similar to the outcomes of previous research (Cheney and Seyfarth 2009). However, there was no significant difference in the baseline cortisol levels between the two species. Thus, baseline cortisol levels cannot explain the observed differences in the stress response to male introductions between the species.

The infanticide risk and other stress-causing factors may also differ between introductions within the same species. Possibly, males differ in their tendency to commit infanticide; not all males from species with high infanticide risk may be infanticidal. 
Additionally, females from longer introductions may be expected to experience more stress due to prolonged social instability. However, we did not find an effect of the duration of the introduction on the change in cortisol. Yet, we had to transform our data into categories, making our analysis less precise. Still, the importance of the duration of the introductions can be questioned, as the first days of an introduction are likely most stressful. These first days are associated with the highest aggression levels and group instability, which decrease quickly over time (Rose et al. 1972; Bernstein et al. 1977; Rox et al. 2018). Females from all introductions therefore experienced a similar brief period with elevated aggression levels. Infanticide risk, however, may be present for several months after male group entry, but is largest during the first three months (Fedigan 2003; Cheney and Seyfarth 2009). Thus, infanticide risk could influence the female stress response during both fast and slow introductions. However, we did not observe any infanticidal attacks during any of the introductions, making it difficult to estimate whether infanticide risk differed between the introductions.

Infanticide risk may not only differ between species and between introductions, it may also differ between individuals. Especially lactating females may be at direct risk of infanticide, as they have dependent offspring. We indeed found that lactating females experienced more long-term stress during the introductions than females without dependent offspring. This fits with other studies that only report a significant increase in short-term stress in and lactating females (Beehner et al. 2005; Engh et al. 2006). The effect of lactation on the change in cortisol in response to the introductions did not depend on species. Possibly, all lactating females, irrespective of the species' infanticide risk, show a long-term stress response to new males. This would fit the observation that infanticide is rare in rhesus macaques, but not unseen (Camperio Ciani 1984; $B P R C$ records), Alternatively, our sample on lactating long-tailed macaques ( $N=3$ ) may be too limited to detect a species difference. Still, it is apparent that especially females at direct risk of infanticide experience long-term stress during introductions.

Females possibly mediate the infanticide risk they experience is through interactions with the new males. Infanticide risk may be linked to proximity and mating behaviour, as these behaviours are part of counterstrategies against infanticide. Long-tailed macaque females, but not rhesus macaque females, that spent more time in proximity of the new male and mated less with him showed larger increases in hair cortisol levels. Females may avoid unfamiliar males to decrease the chances of infanticide (Hrdy 1979; Ebensperger 1998; Beehner et al. 2005). Thus, females that spent more time with new males take more risks and may show an increased stress response. Moreover, infanticide risk would decrease if a female mates more with the new male due to paternity confusion (Hrdy 1977, 1979; Ebensperger 1998). Our results indeed show that females that avoid the new male or mate more with him experience less stress. It is important to realize that the animals had opportunities to avoid other individuals in their group and were not forced to interact with the new males during the introductions. Thus, the observed effect of proximity on stress in long-tailed macaques was due to voluntary proximity to the male, and did not results from unwanted forced interactions. 
However, only females at risk of infanticide are expected to show counterstrategies, and could use them to decrease infanticide risk and thereby alleviate less stress. It remains unclear why this very strong effect has been found in when considering all long-tailed macaque females in our sample. Possibly, many long-tailed macaque females may have perceived an infanticide risk, as they mate promiscuously and it is highly likely they mated with the resident males before the new male was introduced. Therefore, they could potentially be in the early stage of pregnancy. This potential pregnancy, and thereby potential infanticide risk, may have triggered a long-term stress response in most long-tailed macaque females. Therefore, the difference in perceived infanticide risk could explain the effect of counterstrategies on the stress response of all long-tailed macaques. There was no effect of proximity and mating on the stress response in female rhesus macaques. Since the infanticide risk is low in this species, counterstrategies to infanticide are expected to be far less common in rhesus macaques. These results therefore support the notion that infanticide risk is an important factor mediating the female stress response to male group entry.

In the long-tailed macaques, another possible counterstrategy against infanticide was observed. One of the females, Kaloa, aborted her fetes during the introduction of the male, when she was approximately 4 months pregnant. Such an abortion may be the result of exposure to an unfamiliar male, the so-called Bruce effect (Bruce 1960; Hrdy 1979; Ebensperger 1998; Roberts et al. 2012; Amann et al. 2017). The Bruce effect is an effective counterstrategy as it prevents further investment in offspring that is likely to suffer from infanticide. Surprisingly, Kaloa's cortisol levels decreased drastically during the introduction with more than $20 \mathrm{pg} / \mathrm{mg}$ hair (long-tailed macaque average: $9.36 \mathrm{pg} / \mathrm{mg}$ hair increase). Possibly, cortisol levels decrease drastically after abortion during male group entry, due to a quick decrease in infanticide risk. More data on hair cortisol levels of females aborting their fetes during male group entry are needed to verify this hypothesis. However, Kaloa's abortion is another valuable indication that infanticide risk may be of great importance in determining a female's stress response during male group entry.

\section{Social buffering}

In species where infanticide is uncommon, we expected a role for social buffering in the stress response to male introduction. The effect of buffering may be overruled by the infanticide risk in species where infanticide is common. Surprisingly, we found an effect of social buffering on the stress response in both female long-tailed macaques and rhesus macaques. However, the effects contradicted each other. Female longtailed macaques with a more dispersed social network experienced less stress during the introduction than females with a more focused social network, while female rhesus macaques with a more dispersed social network experienced more stress. First, the results indicate that social buffering is not overruled by the infanticide risk in longtailed macaque females, and stresses the importance of social bonds. However, the results of the long-tailed macaques contrast with the common view on social buffering. Several studies found that having strong bonds, indicated by a focused social network, 
buffers stress (Crockford et al. 2008; Wittig et al. 2008), similar to the pattern we found in rhesus macaques. Possibly, infanticide risk may explain the opposite effects in the species. Having a good male friend may help buffering stress during male group entry because male friends can protect females against infanticide (Hrdy 1979; Palombit et al. 1997; Palombit 2009). The groups in our study lack resident adult males, while individual female friends may provide little protection against infanticidal males. However, females regularly form coalitions against new males and may join together to protect group members (Bernstein et al. 1977; Packer and Pusey 1979; Lukas and Huchard 2014). Female long-tailed macaques with a more dispersed network may have more friends and thereby a larger coalition that provides better protection against infanticide (reviewed in Ebensperger 1998). As infanticide risk is low in rhesus macaques, strong bonds may buffer stress in this species, similar to what is described in other studies (Crockford et al. 2008; Wittig et al. 2008). Finally, the presence of close kin did not provide a social buffer to stress. This is in line previous studies (Abbott et al. 2003; Crockford et al. 2008; Setchell et al. 2008), and indicates that social bonds with non-kin may be equally important to macaques as the presence of kin. Overall, it is the social network that buffers stress. Yet, whether a dispersed or focused social network is better may differ between species that differ in their infanticide risk.

Finally, the social network of rhesus macaques, but not the long-tailed macaques, was related to baseline cortisol levels. Females with high baseline cortisol levels had a more focused social network. Both factors also affected the change in cortisol. Possibly, high baseline cortisol levels result in a more focused social network in an attempt to buffer stress, which leads to a lower stress response to male introductions. Unfortunately, we cannot determine how these factors interact with each other due to our limited sample size. There was no relationship between any of the factors affecting the change in cortisol in the different analysis for the long-tailed macaques. This implies that all factors that affect the change in cortisol in response to male introduction in long-tailed macaques act independently of each other. Thus, lactating females did not display more behaviour linked counterstrategies to infanticide than pregnant of cycling females. This fits with our prediction that all long-tailed macaque females are at potential risk of infanticide, making them equally likely to display counterstrategies. Whether this difference between the species can also be linked to infanticide risk remains unclear. Data on additional introductions are needed to draw decisive conclusions on the interactions between individual characteristics, infanticide risk, social behaviour and long-term stress.

\section{Conclusion}

To conclude, male introductions caused more long-term stress for females in a species with a high infanticide risk (i.e. long-tailed macaques) than for a species with low infanticide risk (i.e. rhesus macaques). Yet, the presence of this species difference needs to be confirmed with a larger dataset. We showed that introductions were more stressful for females that are at direct risk of infanticide. Moreover, within the species with the high risk of infanticide, displaying female counterstrategies against infanticide 
was linked to relatively low long-term stress levels. Finally, we observed an abortion, which is possibly a counterstrategy against infanticide. Altogether, these results support the hypothesis that the female long-term stress response to male group entry may depend on infanticide risk. Thus, not only infanticide itself may affect a female's fitness during male group entry, but also the risk of infanticide may induce a long-term stress response with negative fitness consequences. Thereby, this preliminary study implies that infanticide risk may be an important evolutionary force in shaping female responses to new males. 
30
8 



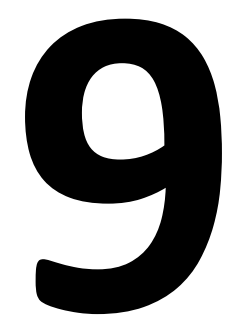

General discussion and summary 


\section{Background and aims}

Dispersal is widespread among the animal kingdom, and is associated with costs and benefits. Dispersing individuals may benefit from decreased chances of inbreeding in their new environment (Moore and Ali 1984; Pusey 1987; Krause and Ruxton 2002), but also often experience high mortality rates due to enhanced predation risk and difficulties to find food and shelter (Isbell 1990; Isbell et al. 1993; Alberts and Altmann 1995; Isbell and van Vuren 1996). For group-living animals, dispersal often entails additional social costs and benefits of entering a new social group, where they have to establish social relationships with new group members (Isbell and van Vuren 1996; Chapter 2). These social costs and benefits of dispersal may be especially apparent in primate species living in multi-male multi-female groups with male biased dispersal, a common social system among primates (Mittermeier et al. 2013; Rowe and Myers 2016). Within these species, new males face the challenge to obtain a new social position in a group with a cohesive core of related females and multiple adult males to compete with. They generally change groups several times during their lives, making the social costs and benefits they experience during group entry important determinants of their lifetime fitness.

Up to now, researchers studying the social costs and benefits associated with male group entry mainly focused on male-male competition during male group entry (e.g. van Noordwijk and van Schaik 1985; Zhao 1994; Marty et al. 2016). Moreover, they generally assume that a new male obtains mating access to females as soon as he succeeds in entering a group. Thereby, they ignored female mate choice, a wellestablished phenomenon among primates (Clutton-Brock and McAuliffe 2009). Moreover, not only male fitness, but also female fitness may be affected when a new male enters a group. Females can therefore be expected to optimize the costs and benefit they experience during male group entry, and actively interact with new males. Females are at risk of severe aggression by new males and may be injured during male group entry (e.g. Pereira 1983; Alberts et al. 1992; Cooper et al. 2001; Fruteau et al. 2010; Brasington et al. 2017). In extreme cases, the injuries may even lead to a female's death or disappearance (Rowell 1974; Fedigan and Jack 2004; Brasington et al. 2017). Moreover, females may be at risk of infanticide during male group entry. Infant mortality may increase drastically when new, potentially infanticidal, males enter a group (Engh et al. 2006; Fruteau et al. 2010; van Belle et al. 2010; Amann et al. 2017). Consequently, females may aggress or avoid unwanted males during group entry. This female-new male aggression may even lead to unsuccessful group entry for a new male (Neville 1968a; Bernstein and Gordon 1974; Bernstein et al. 1977; Packer and Pusey 1979). However, new males may also be preferred mating partners (Packer 1979; Zhao 1994; Manson 1995; Alberts and Altmann 1995; van Belle et al. 2010; Hayakawa and Soltis 2011; Fernández 2017; Rox et al. 2018) of whom females may stimulate group entry. Thereby, female behaviour likely influences the costs and benefits males experience during group entry, and may even affect a male's chances of successful group entry. In contrast to resident male - new male behaviour, female behaviour during male group entry is a neglected topic in literature and has never been 
studied systematically. Yet, it is crucial to gain a clear view on the role of all resident group members during male group entry to fully understand the social costs of male dispersal in primates.

However, it is difficult to conduct systematic studies on male group entry in the wild. Although males change groups several times in their lives, male group entry is unpredictable. Consequently, researchers rarely witness male group entry when observing wild primate groups. Only long-term studies collecting data on several different study groups may collect sufficient data to study social behaviour during male group entry in detail (e.g. van Noordwijk and van Schaik 1985). Studying male group entry in captive groups where natural group composition and dispersal patterns are mimicked may overcome this problem. Complex social behavioural patterns, such as reconciliation behaviour, are similar between such naturalistic captive groups and wild groups (Aureli et al. 1989; Aureli 1992). Therefore, valuable knowledge on female social behaviour during male group entry may be derived from captive studies. Moreover, studying female behaviour during male introductions will provided information necessary to improve captive introduction management and improve primate welfare (Olsson and Westlund 2007).

The research described in this thesis aimed to gain better understanding of femalenew male interactions during male group entry and their fitness consequences in primates, by studying captive male introductions. Introductions of unfamiliar rhesus macaque (Macaca mulatta) and long-tailed macaque ( $M$. fascicularis) males into naturalistic captive groups were studied at the Biomedical Primate Research Centre (BPRC) in Rijswijk, the Netherlands. The introductions concerned necessary management procedures to prevent inbreeding, and allowed us to study male group entry under circumstances closely mimicking the wild. Wild macaques generally live in multi-male multi-female groups, but also one-mail groups are observed (Neville 1968a; Lindburg 1969; van Noordwijk and van Schaik 1985; Ménard and Vallet 1993; Keane et al. 1997; Singh et al. 2006). Similarly, one-male groups were created during the introductions, allowing us to look beyond male-male competition and focus on femalenew male behaviour. Interactions between resident females and their new males were studied from an applied (Chapter 3-5) and fundamental (Chapter 2 and 6-8) point of view. Therefore, the results obtained in this thesis are discussed both from a fundamental and an applied perspective.

\section{Fundamental approach}

In this thesis we first identified the current gaps in knowledge on female-new male behaviour during male group entry in primates. As female behaviour has barely received attention in studies on male group entry, even basic knowledge on female behaviour during male group entry was lacking. Implicitly, females have often been portrayed as passive group members. In contrast, this thesis clearly shows that females can actively promote or avoid interactions with new males, and may play a significant role during male group entry. 
To start, Chapter 2 reviews the information on female-new male interactions present in literature. Some papers focussing on male-male interactions during male group entry also contain a few sentences with information on female behaviour towards new males. This information is often non-quantitative, but forms a starting point to gain understanding of the female role during male group entry. Whenever information on female-new male behaviour is provided in literature, this mostly concerns descriptive information on whether certain interactions are present or absent. In particular, the presence of female-new male aggression and the presence of female-new male mating is described. Generally, female-new male affiliation is overlooked, and it remains unknown how many or which females engaged in certain behaviour with new males. Moreover, the possible fitness consequences of femalenew male interactions are generally ignored. To remedy this gap in our knowledge, the studies within this thesis particularly focus on quantifying all possible female-new male interactions during male group entry, determining the fitness consequences of these interactions between females and their new male, and identifying which females interact with new males and why.

\section{The female response to new males}

In this thesis, female-new male behaviour during male group entry is studied during captive male rhesus macaque (see Chapters 4 to 8 ) and long-tailed macaque (see Chapter 8) introductions at the BPRC. Generally, the observed behavioural patterns are similar between introductions. First, female-new male aggression is generally high at the start of male group entry, but diminishes within several days (Chapter 4). This is in line with previous studies on male group entry in captivity and the wild (Chapter 2). Thereby, females may resist male group entry at first, but cease their defence quickly when they become familiar with the new male.

The same pattern is observed when studying female-new male mating. Males may obtain mating access directly after group entry, and engage in mating with resident females frequently, up to 50 times per hour (Chapter 2, Chapter 4, Chapter 7). Yet, mating rates decrease as the male spends more time with the group (Chapter 2). Females can take initiative for mating and may actively invite new males to mate. They may even initiate mating with new males more often than with resident males (Chapter 2, Chapter 7). These results imply a female preference for mating with new males.

Next, our results show that female-new male submission, including displacement behaviours and the bared teeth that signal submission (cf. Angst 1974), is high at the start of the introductions and decreases over time (Chapter 4). Females may use submission to signal they accept the new male's dominant position in their group. This fits with bluff immigration, which is mimicked during the studied male introductions. New bluff males immediately obtain a position in high in the dominance hierarchy an may receive submission from residents (van Noordwijk and van Schaik 1985). In addition, high rates of submissive signals may indicate socially instable relationships, as submission decreases when the new male establishes a social position in the group (Chapter 4). The only, possibly submissive, behaviour other studies report is female 
avoidance of new males. Females may particularly avoid unwanted males when they are unable to keep him distant from their group using aggression (Chapter 2). Yet, the interpretation of this behaviour may be different, as avoidance may reflect female choice and signal females to not accept a new male in their group.

Next, female-new male affiliation is a particular point of interest in this thesis. Female-new male affiliation has thus far received little attention from researchers (Chapter 2). During our studies, it becomes clear that females actively seek contact with new males, and may even approach new males more often than the new males approach them (Chapter 2, Chapter 4, Chapter 7). Yet, not all females may tolerate new males in their group and engage in affiliation (Chapter 4, Chapter 6). During the introductions studied in this thesis, it becomes clear that female affiliation increases over time as the male established a social position in the new group (Chapter 4). Affiliation may even be one of the most important behaviours during male group entry, as reciprocation of affiliation may be one of the main mechanisms through which social bonds are formed (Chapter 6).

Taken together, it is clear female-new male aggression and mating are high right after male group entry, and may decrease over time. This fits with previous studies reviewed in Chapter 2. Female-new male submission and affiliation were studied far less. We showed that female-new male submission decreases over time, while femalenew male affiliation increases. Yet, these behaviours require more attention in future research. Especially female-new male affiliation may be important as this may signal a new male's integration into the existing social group; a crucial process of male group entry in captivity and the wild.

\section{The fitness consequences of female-new male behaviour}

After gathering information on the general patterns in female-new male behaviour during male group entry, the next step identifies the fitness consequences of these interactions. Obviously, a new male's fitness is affected by dispersal, but also female fitness will be affected during male group entry. Interactions with new males can be risky for females (Rowell 1974; Cooper et al. 2001; Fedigan and Jack 2004; Brasington et al. 2017; Rox et al. 2019; Chapter 2), females are therefore only expected to interact with new males when these interactions result in fitness benefits. The male and female fitness consequences of female-new male aggression, female-new male mating, and female-new male affiliation, and infanticide risk will be discussed.

First, female-new male aggression is related to the costs males experience during group entry. Females may initiate aggression towards new males, increasing the number of conflicts a male engages in; a costly behaviour (Chapter 2). Moreover, females may injure new males, form coalitions that may overpower males, prevent successful male group entry, or even kill new males (Chapter 2, Chapter 4, Chapter 5). Unsuccessful group entry means males invest energy into dispersal and experience high costs of aggression without obtaining any benefits. Yet, female-new male aggression is not always equally high. Female-new male aggression levels may differ between introductions (Chapter 2, Chapter 4), implying that not all females respond 
equally aggressive to all males. They may withhold aggression to preferred males, or increase aggression only towards unwanted males (Chapter 2). Yet, what determines female aggression levels towards new males remains unknown. Moreover, the female fitness consequences of female aggression remain to be studied. Possibly, females may benefit from aggression when they can prevent unwanted males from entering their group or prevent infanticide. However, females displaying aggression may also experience increased costs during male group entry, as new males are likely to display counter aggression. Thus, the female fitness consequences of female-new male aggression remain unclear. In contrast, it is clear female aggression may affect a male's social costs during dispersal, yet, how and by whom female aggression is used to increase these costs needs to be studied in the future.

Second, males may obtain reproductive benefits from mating with females. High mating rates right after male group entry may imply high reproductive benefits for males (Chapter 2, Chapter 4, Chapter 7). Indeed, several studies show that males successfully obtaining the alpha position in a new group obtain reproductive benefits (van Noordwijk and van Schaik 2000; Georgiev et al. 2016). This thesis also shows that new males impregnate the majority of the females during introductions into a new group and sire offspring the next year (Chapter 7, Chapter 8 ). Moreover, BPRC records imply that males sire most offspring in their breeding group during the first two years of residence (personal communication). Thus, males obtain reproductive benefits after group entry through mating with resident females. Females may play an active role in this, as females can promote mating with new males through approaching and sexual invitation (Chapter 7). The effect of these female behaviours on female reproductive success was studied in this thesis. Females competed over access to preferred new males and were found to use mating tactics. These mating tactics resulted in increased mating access, yet did not affect reproduction (Chapter 7), suggesting female-new male mating may not affect female reproductive success. However, in captive conditions fitness consequences for females may be less pronounced, e.g. due to their superior bodily condition. Still, female-new male mating may affect male and female fitness, as researchers suggested mating may underlie social bonds formation and promote successful group entry (Sprague 1992; Olupot and Waser 2005). This is in line with the results obtained in this thesis, showing that males may particularly form bonds with females they mated with early during the introductions (Chapter 6). Thus, both new male and female fitness may be affected by mating as it promotes bond formation, yet, there is no evidence that mating leads to increased female reproductive success in our captive setting.

Third, we study female-new male bond formation during male group entry. The existence of social bonds has been thoroughly studied in primates (reviewed in: Silk 2002; Massen et al. 2010; Seyfarth and Cheney 2012). The presence of same-sex and opposite-sex bonds and their fitness consequences are well described (e.g. Hill 1990; Henzi and Barrett 1999; Cords 2002; Silk et al. 2006; Massen and Sterck 2013; Haunhorst et al. 2016). Yet, we are the first to describe the mechanism through which social bonds between unfamiliar individuals can be formed. We show that female 
tolerance of a new male is crucial for his social integration, as female-new male affiliation increases with social tolerance by females (Chapter 4) and early tolerance leads to the establishment of social bonds (Chapter 6 ). Moreover, female reciprocation of new male affiliation and mating between a female and the new male early during the introductions predict the bonds established after the introductions (Chapter 6). In this process females also play an important role, as female approaches and sexual invitations are linked to mating with new males (Chapter 7). Thus, females play an active role during social bonding, which appears to be a joined effort by females and their new male. The bonds formed during the introductions appear long-lasting, an may therefore have important long-term fitness consequences for both males and females (Chapter 6). Yet, whether these benefits, such as increased mating access for males (Massen et al. 2012) and increased protection for females (Beehner et al. 2005; Sonnweber et al. 2015), were also obtained during our study needs the be established in the future. Additionally, social bonding is important for a male's social integration into the group, and may increase the likelihood of successful group entry (Chapter 2). Taken together, female affiliation and tolerance of new males may have important long-term fitness consequences and increases the benefits of male dispersal through stimulating male group entry.

Fourth, we focus on the fitness consequences of infanticde during male group entry. There was no infanticide observed during any of the introductions studied in this thesis. Consequently, we cannot compare the fitness of infanticidal and non-infanticidal new males. Yet, there is plenty of literature suggesting males may gain additional reproductive opportunities after committing infanticide (e.g. Hrdy 1977, 1979). However, infanticide may also be a costly behaviour to males, as females may form coalitions against infanticidal males and physically attack them (Chapter 2). For females, infanticide is always costly. Infant mortality increases drastically when a new male enters a group (Engh et al. 2006; Fruteau et al. 2010; van Belle et al. 2010; Amann et al. 2017), which forms a large cost for females who have invested a lot of time and energy into their offspring. In this thesis, we show that the risk of infanticide when a new male enters the group may even be sufficient to impose costs for females. Pregnant females may abort their fetes when a new male enters the group, preventing the investment of energy into non-viable offspring, the so-called Bruce effect (Bruce 1960; Roberts et al. 2012). We observed such an abortion during a male introduction in long-tailed macaques (Chapter 8). Moreover, long-tailed macaque females, a species in which infanticide risk is high, experience long-term stress during introductions of a new male. In contrast, rhesus macaque females, that experience lower infanticide risk during male group entry, did not experience long-term stress (Chapter 8). Long-term stress has negative fitness consequences (Charmandari et al. 2005; Honess and Marin 2006; Cheney and Seyfarth 2009). Additionally, especially lactating females, who are at risk of infanticide, experience long-term stress. Females displaying counterstrategies against infanticide, in contrast, may lower the risk of infanticide and experience least stress (Chapter 8). This shows that the risk of infanticide associated with male group entry, and the female response to these new males, may have important fitness consequences. 
In summary, female-new male behaviour can be linked to costs and benefits males and females experience during group entry. Up to now, the consequences of femalenew male interactions were almost never quantified. Within this thesis, we attempted to measure the fitness consequences of female-new male interactions. We show that females may prevent males from entering their group (Chapter 4), females may kill new males (Chapter 4), females may experience long-term stress during male group entry (Chapter 8), or lose their unborn fetes as a counterstrategy to infanticide (Chapter 8). This proofs that female behaviour during male introductions can affect female and new male fitness. Additionally, there are other indications that female-new male behaviour has important fitness consequences as it may lead to costly female-male conflicts (Chapter 4), contributes to bond formation (Chapter 6), determines male mating access (Chapter 7), and female counterstrategies to infanticide can buffer longterm stress (Chapter 8). These results lead to the prediction that female-new male interactions affect both male and female fitness. Yet, this may not always be found, as is illustrated by our finding that female-new male mating does not affect female reproductive success in our study (Chapter 7 ). Thus, some but not all female-new male interactions may have pronounced fitness effects.

However, the fitness consequences on female-new male interactions may be difficult to study in captivity. Careful documentation of all injuries, illnesses, deaths, and births in primate facilities, such as the BPRC, may provide valuable information on the fitness consequences of female behaviour. Yet, the circumstances animals are kept in captivity will affect their fitness. To ensure animal welfare, animals will not be hungry, are protected from predators, and will receive veterinary treatment when they are injured or ill. Moreover, food is abundant, of high quality, and easily accessible. In the wild, high-ranking females have priority of access to food (e.g. Whitten 1983; Foerster et al. 2011). Consequently, they are generally more healthy and stronger than lower ranked females, and have higher fitness (reviwed in: Pusey 2012). In captivity, all animals are potentially equally healthy, enhancing their reproductive success and reducing the chances of mortality. Thereby the fitness effects of female-new male interactions may be blurred in captivity compared to the wild. Consequently, not all fitness consequences of female-new male interactions may be visible during captive studies on male group entry. Still, we present some evidence of fitness consequences of female-new male interactions, related to successful male group entry and infanticide risk, and suggest that females play a key-role in determining the social costs and benefits of male dispersal.

\section{Factors determining the female response}

When studying female-new male behaviour during male group entry, it is important to realize there may be intra-specific variation in the female response to new males. Not every female may respond the same to a new male, and not every new male may elicit the same response. The lack of in depth and quantitative studies on the female response to male group entry make it difficult to understand which females engage in certain interactions with new males and why. Individual characteristics may determine 
the costs and benefits females experience during male group entry, and thereby determine a female's response to a new male (Chapter 2). Therefore, this thesis made effort of quantifying the female response, and systematically reported the number of females interacting with new males and their characteristics.

The female response to new males may depend on several different factors, such as infanticide risk, female reproductive status, female age, female dominance rank, and female-female bonds. First, females at risk of infanticide may behave differently towards new males and display counterstrategies to infanticide. Thereby, they may avoid new males or show increased mating interest (Chapter 8). Additionally, females at risk of infanticide, in particular females that are lactating during male group entry, may behave most aggressive towards new males (Chapter 2). Furthermore, also females who are pregnant during male group entry may show increased resistance to the new male, as indicated by lower introduction success in groups where pregnant females are present (Chapter 5). These females are not at direct risk of infanticide, but their future offspring may be killed by new males that entered their group during their pregnancy. Second, females that are receptive (i.e. not pregnant or lactating) may promote male group entry, as new males may be preferential mating partners (Chapter 2, Chapter 4, Chapter 7). However, in our setting, there was no effect of female fertility signals (i.e. sexual colouration) on when a female accept a new male (Chapter 4). This implies that not the timing of the female fertile phase, but her overall reproductive status may determine her attitude towards new males. Additionally, this may explain why wild rhesus macaque males generally migrate right before or early in the breeding season, when most females are receptive (Lindburg 1969; Drickamer and Vessey 1973; Bernstein et al. 1977). Taken together, this illustrates that infanticide risk and female reproductive state likely affect a female's response to new males.

Next, female age and dominance rank may affect female-new male interactions during male group entry. Our results show that older females generally tolerate new males sooner than younger females (Chapter 4). Thereby, we are the first to show that female age may be important during male group entry. Female dominance rank did, in contrast, not affect female tolerance of new males, or female mating access to new males (Chapter 4, Chapter 7). This contrasts with a previous study, suggesting that high ranking females associate more with new males (Kawai 1960). Yet, we suggest that female dominance rank may not affect female-new male interactions during male group entry, but come at play when the male settles in the group (Chapter 4). This is supported by the observation that only the two highest ranked females in a group maintained a social bond with the new male in the year after he was introduced, while the female-male bonds of lower ranked the introductions disappeared (Chapter 6). Altogether, female age, but not female dominance, seems an important determinant of a female's attitude towards new males. Yet, how widespread the influence of female age is and which behaviours, besides female tolerance, are affected by female age needs to be determined in the future.

Additionally, the existing social network in a group likely affects the female response to new males. Primate females form close social bonds with their family members (Silk 
2002b; Silk et al. 2006). These bonds may help females to deal with stressful situations, such as male group entry (Wittig et al. 2008; Truelove et al. 2017), and thereby mediate their response to new males. When female bonds are very close, new males may have a hard time entering a group (Yamada 1963). Moreover, closely bonded females may form coalitions against new males and thereby prevent successful group entry (Chapter 4, Chapter 5, Bernstein et al. 1977; Packer and Pusey 1979). However, the introductions studied in this thesis are most successful and long-term stable in groups with more females in three or fewer families, implying the presence of strong familial bonds (Chapter 5). Moreover, female long-tailed macaques that maintain social bonds with more individuals experience least stress during our introductions, while female rhesus macaques with stronger and more focussed bonds experience least stress (Chapter 8). Taken together, these results imply that female-female bonds may mediate the female response to new males. Yet, how female-new male interactions are affected by femalefemale bonds, and whether this link differs between species needs to be subject of future studies.

In line with this, some of the female characteristics discussed in this thesis may not only explain intra-specific variation, but may also contribute to inter-specific variation in the female response to new males. First, infanticide risk may not only differ between individuals, but also between species. Females from species with high infanticide risk may be more hostile or fearful towards new males compared to females from species with low infanticide risk (Chapter 2, Chapter 8). Second, females from some species remain in their natal group throughout their lives, while female dispersal is more common in other species (Sterck and Korstjens 2000). Females may particularly intervene with male group entry in species with female philopatry, while females from species with female dispersal may change groups when an unwanted male takes over (Chapter 2). However, in the literature there is only basic understanding of female-new male interactions in two, closely related, primate species; rhesus macaques and Japanese macaques (Macaca fuscata). This makes it hard to draw decisive conclusions on factors causing inter-specific variation.

Finally, also male characteristics may determine how females respond to a new male. Male crested macaques (Macaca nigra) may delay their dispersal to reach optimal body condition before entering a new group (Marty et al. 2016). Such heavy, fully-grown, prime aged males are generally most successful in obtaining the alpha position in a new group (Boelkins and Wilson 1972; Packer 1979; van Noordwijk and van Schaik 1985; Sprague 1992; Suzuki et al. 1998; Marty et al. 2017). Similar results were found during the introductions studied in this thesis (Chapter 5). Possibly, these new males are better liked by females, who generally prefer mating with strong and high-quality males (Clutton-Brock and McAuliffe 2009). Alternatively, stronger males may have increased chances of winning conflicts from resident males and females, and obtain the alpha position in this manner. However, fighting ability alone may not be sufficient to become the alpha male and maintain this position in a new group. This thesis shows that males need to develop social skills in their natal group, before they can successfully maintain the alpha position in a group (Chapter 5). These skills may concern restraint in aggression 
and the ability to form affiliative relationships (Kempes et al. 2008). Taken together, this implies that the female attitude towards strong, prime aged, and socially capable males benefits these males. However, whether females indeed behave differently towards strong, prime aged, or socially capable males, compared to weaker, young, or socially impaired males needs to be studied in the future.

In summary, this thesis shows that infanticide risk, female reproductive state, female age, the female social network in a group, male body condition, and a male's social skills may determine how individual females respond to a new male. Moreover, infanticide risk and the female tendency to disperse may explain differences in femalenew male behaviour between species. This information is the first step in understanding why females may intervene with male group entry. It is important to realize that there may be additional factors affecting the female response to new males. It is impossible to cover all factors affecting the female response to male group entry in one thesis when even the most basic information on female-new male information is lacking. Moreover, which behaviours are affected by which factor is not yet fully understood. Additionally, information on the female response to new males is lacking in many different species, making it impossible to make a reliable comparison between species. Therefore, more systematic studies reporting which females engage in which interactions with new males in a wide variety of species, are called for.

\section{Future prospective}

Within this thesis, we tackled most of the gaps in knowledge that appeared after reviewing the available literature on female behaviour during male group entry (see Chapter 2). We conducted systematic and quantitative studies on female behaviour during male group entry (see Chapter 4, Chapter 6, Chapter 7, Chapter 8), while especially focussing on the behaviours potentially benefitting male and female fitness (see Chapter 6, Chapter 7), identified factors affecting the female response to new males (see Chapter 5, Chapter 8), and compared the female response between species (see Chapter 2, Chapter 8). Thereby, we showed that females are active contributors to, and not passive onlookers in, the process of male group entry. Yet, hopefully this thesis only represents a starting point of many more studies on the female role during male group entry.

When female behaviour during male group entry is fully understood, the next step is identifying which costs and benefits are the result of female-new male interactions, and which costs and benefits are the result of resident male-new male interactions. Moreover, the interaction between female behaviour and the behaviour of new or resident males needs to be studied. Females may respond differently to new males when resident males are nearby (Neville 1968b). Additionally, the female response to a new male may depend on new male behaviour (Yamada 1971; Winston 1985), such as his strategy during group entry (i.e. bluff or unobtrusive (van Noordwijk and van Schaik 1985)). This is supported by our finding that males that matured in their natal group, where they gain valuable social skills, are more successful in obtaining a longterm stable position in a new group (Chapter 5). Yet, how female behaviour is affected 
by resident or new male behaviour remains to be studied. Understanding the interaction between female and male behaviour during group entry is crucial to fully understand the costs of male dispersal in species living in multi-male multi-female groups, such as macaques. The social costs and benefits of male dispersal within these species will be the sum of all costs and benefits new males derive from interactions with all resident group members. Yet, in the wild, it will be difficult to disentangle these female or male inflicted costs and benefits. Here, studies on male introductions creating one-male groups in captivity may also proof valuable in the future.

To conclude, female-new male behaviour during male group entry should receive systematic attention in future studies. We have shown that female-new male interactions are important and females may play a key-role in determining the costs, benefits, and success of male group entry. Still, studies on a wide variety of species, describing the presence and absence of all possible interactions between resident females and new males, are needed. Only if we understand how females behave during male group entry, why they do so, and the fitness consequences of female-new male interactions are clear, can we truly understand the fitness consequences of male dispersal for both males and females. Thus, females should not be ignored; they are not passive onlookers during male group entry. Instead, they may actively influence who is entering their group and who is not.

\section{Applied approach}

Studying female behaviour during male introductions is crucial for improving the behavioural management strategies of captive primate groups, and enhance animal welfare. Animal welfare can be increased through promoting the expression of natural species typical behaviour (Olsson and Westlund 2007; Fraser 2008). As primates are highly social animals, mimicking natural group composition and socio-dynamics is crucial to ensure primate welfare and successfully manage captive primate groups (Olsson and Westlund 2007). However, even when nature is mimicked closely, managing captive primate groups is challenging. Some necessary management procedures, such as the introduction of new males into existing social groups to prevent inbreeding, may be risky.

This thesis elaborates on some of the risks associated with male introductions, and dedicated three chapters to improving male introduction procedures (see Chapter 3-5). Our results show that male introductions are associated with high levels of female-new male aggression (Chapter 4) similar to what is observed during male group entry in the wild (Chapter 2). Additionally, new males introduced into a social group may be rejected by the females. Females may prevent successful male introduction in $23 \%$ of the cases, and even kill unwanted new males in extreme cases (Chapter 5). Moreover, females may experience long-term stress during male introductions (Chapter 8). Consequently, male introductions may compromise animal welfare. However, male introductions remain necessary to prevent inbreeding in groups with female philopatry. Optimizing male introduction management may minimize the risks associated with male introductions. 
Two different approaches are used in this thesis to determine how male introduction management can be improved. First, we aim to improve male-to-group matching through analysing which males perform best during introductions, and which groups are most acceptant of new males. During this analysis, we not only consider short-term introduction success but introduced research into long-term stability (i.e. 4- 5 years of stability; see Chapter 4). The overall aim in a breeding colony should be minimizing the number of potentially risky introductions and increase long-term group stability. Groups only need to experience a male introduction every 4 to 5 years when males are able to maintain a long-term stable position in the group and can be removed for management reasons (e.g. preventing inbreeding). Moreover, maintaining social stability will lead to lower levels of aggression, stress and injuries (Sapolsky 1983, 1992; Shively et al. 1986; Crockford et al. 2008). Therefore, primate management programs should not only focus on introduction success, but also on forming long-term compatible groups (Chapter 4, Chapter 5). We conduct a retrospective analysis of all male rhesus macaque introductions that occurred at the BPRC between 2003 and 2018. We show that introduction success and long-term stability levels during our male introductions are comparable to the success levels obtained after pair-formation; a less socially complex procedure (Chapter 5 ). We therefore consider the male introduction procedure used at the BPRC a good example of how captive primate groups should be managed (see Chapter 3). Moreover, we identify the male and female characteristics that predict introduction success and long-term stability. We show that male introductions were most successful and long-term stable when the natural group composition and migration patterns observed in the wild are more closely mimicked (Chapter 5). This is in line with the idea that animal welfare can be optimized when natural behaviour is stimulated (Olsson and Westlund 2007; Fraser 2008).

Yet, some gaps in knowledge remain. The sample size of the retrospective analysis of male introductions this thesis covers $(\mathrm{N}=64)$ is too limited to identify a possible interaction between the male and female characteristics. Moreover, future studies could focus on other risks associated with male introductions, and identify which male and female characteristics are related to increased levels of injuries or long-term stress during introductions. This information is valuable to further optimize male introduction success and improve animal welfare. Still, our results are an important first step to decrease the risks associate with male introduction and show that it is best to manage captive primate groups as naturally as possible.

Second, we aim to improve the behavioural monitoring of male introductions and identify cues that may signal the progress or future success of an introduction. According to the experts conducting male introductions at the BPRC, it is crucial to intervene with unsuccessful introductions in time to prevent resident females socially defeating new males. Socially defeated males may be severely or deadly injured by females, and will not be able to obtain the alpha position in another social group (Chapter 3, Chapter 4). Therefore, the BPRC introduction experts stop the introduction when they are in doubt whether the introduction progresses well and has a decent chance of success. Up to now, they do this based on personal knowledge and 
experienced (Chapter 3). Within this thesis, we aim to develop quantitative measures to determine how introductions progress and whether they may succeed. We show that some aggression is normal during introductions, but this should diminish relatively quickly (Chapter 4). When aggression remains high, introductions may fail or be unstable in the long run (Chapter 3, Chapter 4). Moreover, high levels of submission during introductions may signal fear or social instability, while low levels of female-new male submission may signal that the new male's position in a group is settled and accepted by the females and it is safe to leave a male in the group full-time (Chapter 4). It is important to note that new males should never show submission to resident females, if a male does show submission the introduction should be stopped immediately (Chapter 3). However, not only agonistic interactions between females and their new male should be monitored. There should also be attention for positive social interactions, such as social tolerance and affiliation. Our results show that tolerance by only part of the females in a group is sufficient for successful and longterm stable introductions (Chapter 4). The minimum number of tolerating females necessary for introduction success remains to be determined, yet, the BPRC introduction experts only leave a male in the group full-time when at least $50 \%$ of the adult females tolerates the new male (Chapter 3 ). Therefore, introductions should be carefully planned and executed, and female-new male interactions should be closely monitored. Especially female-new male aggression and female-new male submission may be important signals of introduction success and long-term stability.

This thesis contains behavioural data on five male rhesus macaque introductions that were all successful, and of which three were long-term stable. In the future, we hope to be able to include behavioural data of unsuccessful introductions into the dataset, and directly compare the behavioural patterns between unsuccessful and successful introductions. This may provide more certainty about the behaviours signalling introduction success. Moreover, being able to identify individual females that pose a particular risk to new males will be a helpful tool to manage introductions. Introductions into group containing such high-risk females may need extra time or precautions, and these females may be individually familiarized with the new male before the male is physically introduced into a group (Chapter 3 ) to increase introduction success. This question may be linked to the fundamental research aiming to identify which female characteristics determine the female response to new males. In particular, pregnant females or younger females may be most resistant to newly introduced males (Chapter 4, Chapter 5). Moreover, there may be species differences in the risks associated with male introductions. This thesis particularly focused on male introductions in rhesus macaques, which can be considered relatively risky. The BPRC uses a similar introduction procedure for their long-tailed macaque groups, and introductions into groups of this species are always successful (unpublished data from the BPRC database). Thus, there may be inter- and intraspecific variation in the risk females pose during introductions. Understanding the causes and consequences of this variation may help to further improve male introduction management in the future. 
In summary, the best way to maintain long-term stable captive primate breeding groups in through naturalistic group housing. This way, primate welfare is optimized as they are able to express natural complex social behaviour and learn crucial social skills, while inbreeding can be prevented through closely mimicking natural socio-dynamics. The risk of necessary male introductions into naturalistic groups can be minimized by carefully planning and monitoring the introductions. In general, the more closely the management procedures mimic nature, the more successful the introductions and the more long-term stable the group.

\section{Are peer groups a good alternative to naturalistic group housing and male introductions?}

Still, even carefully managed introductions can be risky. The risk of injuries and longterm stress remain, even during successful introductions (e.g. Chapter 8). Therefore, alternative methods of preventing inbreeding in naturalistic primate groups can be considered. One alternative way to prevent inbreeding is removing the offspring of a breeding male from the group before they reach adulthood. This will diminish the risk of inbreeding between a breeding male and his maturing daughters, and thereby obviate male introductions. Many research institutes world-wide use this strategy to manage their primate groups. The current norm for weaning offspring from their mother and transferring them to peer-groups is at an offspring age of 10-14 months (Prescott et al. 2012). Yet, peer-reared individuals experience high levels of stress early in life, which has long-term consequences on their behavioural development (e.g. Veenema 2009). Moreover, they may show increased levels of aggression, submission and abnormal behaviours, and respond with aggression to nonthreatening situations later in life (Mineka and Suomi 1978; Kempes et al. 2008). This shows they are socially incompetent and their welfare may be at stake. Moreover, breeding groups will lack natural matrilines when offspring is reared in peer-groups. These matrilines are a keyfeature of wild primate groups. Females within a matriline form close social bonds, support each other in conflicts, and defend their dominance rank together (Silk 2002b; Suomi 2005; Silk et al. 2006; Oates-O'Brien et al. 2010). Our research shows that groups with few large matrilines, resembling wild primate groups, are more socially stable (Chapter 5), which is supported by previous research (McCowan et al. 2018). Moreover, males removed from their natal group before the age they would leave their natal group in the wild, performed worse during introductions (Chapter 5), suggesting that they lack social competence. Finally, individuals that are weaned too early may be less able to cope with stressful events later in life (Pryce et al. 2002; Veenema 2009), while an altered stress response may interfere with experimental findings during biomedical studies (Barnett 1976). Consequently, proper social development of experimental animals is important for every scientist. Altogether, this shows that removing offspring from their natal group before reaching adulthood compromises animal welfare, is not a good way to prevent inbreeding in primate groups, and may interfere with biomedical research outcomes. Therefore, peer-rearing is not a viable alternative to male introductions to prevent inbreeding. Thus, macaque females should 
remain in their natal group during their lives to form matrilines, and males should not be removed from their natal group before the age they would naturally disperse.

\section{Contribution to the 3R's}

The BPRC houses its primates in naturalistic breeding groups where females remain with female kin and males leave at pubertal age (Chapter 3). Yet, to date, many research institutes still house their primates in non-naturalistic groups and rear offspring in peer-groups from about one year of age. The knowledge obtained in this thesis emphasizes that it is important to mimic nature closely to optimize management procedures and enhance animal welfare. These naturalistic breeding groups may improve the quality life of several hundred thousand of primates used for biomedical research world-wide each year (e.g. Grimm 2018). Additionally, applying this knowledge will provide better experimental animals, which may reduce the number animals needed to obtain significant and meaningful results in biomedical studies. Research already acknowledged several decades ago that abnormal rearing conditions may intervene with experimental results (Barnett 1976). Consequently, naturalistic group housing may contribute to the refinement and reduction of animal experiments.

\section{Concluding remarks}

This thesis represents the first important step in revealing the female role in male group entry. Our studies showed that female-new male interactions can affect the costs, benefits and success of male group entry. This information is crucial to understand the social costs and benefits of male dispersal. Moreover, understanding of femalenew male interactions is necessary to optimize captive male introduction management. We show that mimicking nature closely improves introduction success and the longterm stability of a male's position in the group. Yet, some gaps in knowledge remain. More quantitative studies on female behaviour during male group entry are called for, focussing on the factors determining the female response to new males and fitness consequences for both males and females of female-new male interactions in a wide variety of species. Still, it is clear that female are active contributors to, and not passive onlookers in, the process of male group entry. The key-role females play during male group entry should receive systematic attention by researchers in the future, starting today. 
708
9 



\section{References}

Abbott DH, Keverne EB, Bercovitch FB, et al (2003) Are subordinates always stressed? A comparative analysis of rank differences in cortisol levels among primates. Horm Behav 43:67-82 . doi: 10.1016/S0018-506X(02)00037-5

Albers M, Widdig A (2013) The influence of kinship on kamiliar natal migrant rhesus macaques (Macaca mulatta). Int J Primatol 34:99-114 . doi: 10.1007/s10764-012-9651-y

Alberts SC (2012) Magnitude and sources of variation in male reproductive performance. In: Mitani JC, Call J, Kappeler PM, et al. (eds) The evolution of primate societies. The University of Chicago Press, Chicago and London, pp 412-431

Alberts SC, Altmann J (1995) Balancing costs and opportunities: Dispersal in male baboons. Am Nat 145:279-306 doi: $10.1086 / 285740$

Alberts SC, Sapolsky RM, Altmann J (1992) Behavioral, endocrine, and immunological correlates of immigration by an aggressive male into a natural primate group. Horm Behav 26:167-178 . doi: 10.1016/0018-506X(92)90040-3

Altmann SA (1962) A field study of the sociobiology of rhesus monkeys, Macaca mulatta. Ann N Y Acad Sci 102:338435. doi: 10.1111/j.1749-6632.1962.tb13650.x

Amann AL, Pines M, Swedell L (2017) Contexts and consequences of takeovers in hamadryas baboons: Female parity, reproductive state, and observational evidence of pregnancy loss. Am J Primatol 79:1-8 . doi: 10.1002/ ajp.22649

Angst W (1973) Pilot experiments to test group tolerance to a stranger in wild Macaca fascicularis. Am J Phys Anthropol 38:625-630 . doi: 10.1002/ajpa.1330380278

Angst W (1974) Das Ausdrucksverhalten der Javaneraffen. Ethology 15:1-90

Angst W, Thommen D (1977) New data and discussion of infant killing in old world monkeys and apes. Folia Primatol 27:198-229

Archie EA, Chiyo PI (2012) Elephant behaviour and conservation: Social relationships, the effects of poaching, and genetic tools for management. Mol Ecol 21:765-778 . doi: 10.1111/j.1365-294X.2011.05237.x

Archie EA, Tung J, Clark M, et al (2014) Social affiliation matters: Both same-sex and opposite-sex relationships predict survival in wild female baboons. Proc R Soc B Biol Sci 281:20141261 . doi: 10.1098/rspb.2014.1261

Arlet ME, Grote MN, Molleman F, et al (2009) Reproductive tactics influence cortisol levels in individual male graycheeked mangabeys (Lophocebus albigena). Horm Behav 55:210-216 . doi: 10.1016/j.yhbeh.2008.10.004

Arlet ME, Isbell LA, Molleman F, et al (2014) Maternal investment and infant survival in gray-cheeked mangabeys (Lophocebus albigena). Int J Primatol 35:476-490 . doi: 10.1007/s10764-014-9754-8

Arlet ME, Molleman F, Chapman C (2007) Indications for female mate choice in grey-cheeked mangabeys Lophocebus albigena johnstoni in Kibale National Park, Uganda. Acta Ethol 10:89-95 . doi: 10.1007/s10211007-0034-x

Aschwanden J, Gygax L, Wechsler B, Keil NM (2008) Social distances of goats at the feeding rack: Influence of the quality of social bonds, rank differences, grouping age and presence of horns. Appl Anim Behav Sci 114:116131. doi: 10.1016/j.applanim.2008.02.002

Aureli F (1992) Post-conflict behaviour among wild long-tailed macaques (Macaca fascicularis). Behav Ecol Sociobiol 31:329-337. doi: 10.1007/BF00177773

Aureli F, Cords M (2000) Reconciliation and relationship qualities. In: Aureli F, de Waal FBM (eds) Natural Conflict Resolution. University of California Press, Los Angeles, California, pp 177-198

Aureli F, Schaik CP va. (1991) Post-conflict Behaviour in Long-tailed Macaques (Macaca fascicularis): II. Coping with the Uncertainty. Ethology 89:101-114 . doi: 10.1111/j.1439-0310.1991.tb00297.x

Aureli F, Van Schaik CP, Van Hooff JARAM (1989) Functional aspects of reconciliation among captive long-tailed macaques (Macaca fascicularis). Am J Primatol 19:39-51 . doi: 10.1002/ajp.1350190105

Ballou JD, Lees C, Faust LJ, et al (2010) Demographic and genetic management of captive populations. In: Kleiman DG, Thompson K, Kirk-Baer C (eds) Wild mammals in captivity: principles and techniques for zoo management, 2nd edn. University of Chicago Press, Chicago, p 219

Baniel A, Cowlishaw G, Huchard E (2016) Stability and strength of male-female associations in a promiscuous primate society. Behav Ecol Sociobiol 70:761-775 . doi: 10.1007/s00265-016-2100-8 
Baniel A, Cowlishaw G, Huchard E (2018) Context dependence of female reproductive competition in wild chacma baboons. Anim Behav 139:37-49 . doi: 10.1016/j.anbehav.2018.03.001

Barnett SA (ed) (1976) The Rat- A study in behaviour, Revised ed. Australian National University Press, Canberra

Bashaw MJ, Gullott RL, Gill EC (2010) What defines successful integration into a social group for hand-reared chimpanzee infants? Primates 51:139-147 . doi: 10.1007/s10329-009-0176-8

Bastian ML, Sponberg AC, Suomi SJ, Higley JD (2003) Long-term effects of infant rearing condition on the acquisition of dominance rank in juvenile and adult rhesus macaques (Macaca mulatta). Dev Psychobiol 42:44-51. doi: 10.1002/dev.10091

Bauer SA, Arndt TP, Leslie KE, et al (2011) Obesity in rhesus and cynomolgus macaques: A comparative review of the condition and its implications for research. Comp Med 61:514-526. doi: 10.2133/dmpk.dmpk-11-nt-026

Baxter MJ, Fedigan LM (1979) Grooming and consort partner selection in a troop of Japanese monkeys (Macaca fuscata). Arch Sex Behav 8:445-458 . doi: 10.1007/BF01541200

Beehner JC, Bergman TJ, Cheney DL, et al (2005) The effect of new alpha males on female stress in free-ranging baboons. Anim Behav 69:1211-1221 . doi: 10.1016/j.anbehav.2004.08.014

Berard J (1999) A four-year study of the association between male dominance rank, residency status, and reproductive activity in rhesus macaques (Macaca mulatta). Primates 40:159-175 . doi: 10.1007/BF02557708

Bercovitch FB (1991) Mate selection, consortship formation, and reproductive tactics in adult female savanna baboons. Primates 32:437-452 . doi: 10.1007/BF02381935

Bercovitch FB (1995) Female cooperation, consortship maintenance, and male mating success in savanna baboons. Anim Behav 50:137-149 . doi: 10.1006/anbe.1995.0227

Bercovitch FB (1997) Reproductive strategies of rhesus macaques. Primates 38:247-263

Berghänel A, Ostner J, Schröder U, Schülke O (2011) Social bonds predict future cooperation in male Barbary macaques, Macaca sylvanus. Anim Behav 81:1109-1116 . doi: 10.1016/j.anbehav.2011.02.009

Bergman TJ, Beehner JC, Cheney DL, et al (2005) Correlates of stress in free-ranging male chacma baboons, Papio hamadryas ursinus. Anim Behav 70:703-713 . doi: 10.1016/j.anbehav.2004.12.017

Bernstein IS (1971) The influence of introductory techniques on the formation of captive mangabey groups. Primates 12:33-44 . doi: 10.1007/BF01730380

Bernstein IS (1969) Introductory techniques in the formation of pigtail monkey troops. Folia Primatol 10:1-19

Bernstein IS, Ehardt CL (1985) Age-sex differences in the expression of agonistic behavior in rhesus monkey (Macaca mulatta) groups. J Comp Psychol 99:115-132 . doi: 10.1037/0735-7036.99.2.115

Bernstein IS, Gordon TP (1974) The function of aggression primate societies: Uncontrolled aggression may threaten human survival, but aggression may be vital to the establishment and regulation of primate societies and sociality. Am Sci 62:304-311

Bernstein IS, Gordon TP, Rose RM (1983) The interaction of hormones, behavior, and social context in nonhuman primates. In: Hormones and Aggressive Behavior. Springer, Boston, MA, pp 535-561

Bernstein IS, Mason WA (1963) Group formation by rhesus monkeys. Anim Behav 11:28-31 . doi: 10.1016/00033472(63)90004-6

Bernstein IS, Rose RM, Gordon TP (1977) Behavioural and hormonal responses of male rhesus monkeys introduced to females in the breeding and non-breeding seasons. Anim Behav 25:609-614 . doi: 10.1016/00033472(77)90111-7

Bissonnette A, Bischofberger N, van Schaik CP (2011) Mating skew in Barbary macaque males: The role of female mating synchrony, female behavior, and male-male coalitions. Behav Ecol Sociobiol 65:167-182 . doi: 10.1007/ s00265-010-1023-z

Boelkins RC, Wilson AP (1972) Intergroup social dynamics of the Cayo Santiago rhesus (Macaca mulatta) with special reference to changes in group membership by males. Primates 13:125-139 . doi: 10.1007/BF01840875

Borries C (1997) Infanticide in seasonally breeding multimale groups of Hanuman langurs (Presbytis entellus) in Ramnagar (South Nepal). Behav Ecol Sociobiol 41:139-150 . doi: 10.1007/s002650050373

Borries C, Sommer V, Srivastava A (1991) Dominance, age, and reproductive success in free-ranging female hanuman langurs (Presbytis entellus). Int J Primatol 12:231-257 . doi: 10.1007/BF02547586 
Bracke MBM, Hopster H (2006) Assessing the importance of natural behavior for animal welfare. J Agric Environ Ethics 19:77-89 . doi: 10.1007/s10806-005-4493-7

Brasington LF, Wikberg EC, Kawamura S, et al (2017) Infant mortality in white-faced capuchins: The impact of alpha male replacements. Am J Primatol 79:e22725 . doi: 10.1002/ajp.22725

Braun A, Bugnyar T (2012) Social bonds and rank acquisition in raven nonbreeder aggregations. Anim Behav 84:1507-1515 . doi: 10.1016/j.anbehav.2012.09.024

Brent L, Kessel AL, Barrera H (1997) Evaluation of introduction procedures in captive chimpanzees. Zoo Biol 16:335-342 . doi: 10.1002/(SICI)1098-2361(1997)16:4<335::AID-ZOO5>3.0.CO;2-B

Brent LJN, Semple S, Dubuc C, et al (2011) Social capital and physiological stress levels in free-ranging adult female rhesus macaques. Physiol Behav 102:76-83 . doi: 10.1016/j.physbeh.2010.09.022

Bruce HM (1960) A block to pregnancy in the mouse caused by proximity of strange males. J Reprod Fertil 1:96-103 . doi: 10.1530/jrf.0.0010096

Cameron EZ, Setsaas TH, Linklater WL (2009) Social bonds between unrelated females increase reproductive success in feral horses. Proc Natl Acad Sci 106:13850-13853 . doi: 10.1073/pnas.0900639106

Campbell A (2008) Attachment, aggression and affiliation: The role of oxytocin in female social behavior. Biol Psychol 77:1-10 . doi: 10.1016/j.biopsycho.2007.09.001

Camperio Ciani A (1984) A case of infanticide in a free-ranging group of rhesus monkeys (Macaca mulatta) in the Jackoo. Primates 25:372-377

Carne C, Wiper S, Semple S (2011) Reciprocation and interchange of grooming, agonistic support, feeding tolerance, and aggression in semi-free-ranging Barbary macaques. Am J Primatol 73:1127-1133 . doi: 10.1002/ajp.20979

Charmandari E, Tsigos C, Charmandari E, et al (2005) Endocrinology of the stress response. Annu Rev Physiol 67:259-284 . doi: 10.1146/annurev.physiol.67.040403.120816

Cheney D, Seyfarth R (1982) Recognition of individuals within and between groups of free ranging vervets. Am Zoo 22:519-529

Cheney DL, Seyfarth RM (1983) Nonrandom dispersal in free-ranging vervet monkeys: social and genetic consequences. Am Nat 122:392-412 . doi: 10.1086/284142

Cheney DL, Seyfarth RM (2009) Stress and coping mechanisms in female primates. Adv Study Behav 39:1-44 . doi: 10.1016/S0065-3454(09)39001-4

Clarke AS, Czekala NM, Lindburg DG (1995) Behavioral and adrenocortical responses of male cynomolgus and liontailed macaques to social stimulation and group formation. Primates 36:41-56 . doi: 10.1007/BF02381914

Clarke MR (1983) Infant-killing and infant disappearance following male takeovers in a group o. Am J Primatol $5: 241-247$

Clarke MR, Glander KE (2010) Secondary transfer of adult mantled howlers (Alouatta palliata) on Hacienda La Pacifica, Costa Rica: 1975 - 2009. Primates 51:241-249 . doi: 10.1007/s10329-010-0195-5

Clutton-Brock TH, Albon SD, Gibson RM, Guinness FE (1979) The logical stag: adaptive aspects of fighting in red deer (cervus elaphus I.). Anim Behav 27:211-225

Clutton-Brock TH, McAuliffe K (2009) Female mate choice in mammals. Q Rev Biol 84:3-27 . doi: 10.1086/596461

Clutton-Brock TH, Parker GA (1995) Sexual coercion in animal soscieties. Anim Behav 49:1345-1365

Cooper MA, Bernstein IS, Fragaszy DM, de Waal FBM (2001) Integration of new males into four social groups of tufted capuchins (Cebus apella). Int J Primatol 22:663-683 . doi: 10.1023/A:1010745803740

Cords M (2012) The behavior, ecology, and social evolution of cercopithecne monkeys. In: Mitani JC, Call J, Kappeler PM, et al. (eds) The Evolution of Primate Societies. University of Chicago Press, Chicago and London, pp 91-112

Cords M (2002) Friendship among adult female blue monkeys (Cercopithecus mitis). Behaviour 139:291-314 . doi: $10.1163 / 156853902760102681$

Cowlishaw G, Dunbar RIM (1991) Dominance rank and mating success in male primates. Anim Behav 41:1045-1056 doi: 10.1016/S0003-3472(05)80642-6

Crockett C, Sekulic R (1984) Infanticide in red howler monkeys (Alouatta seniculus). In: Hausfater G, Hrdy S (eds) Infanticide: comparative and evolutionary perspectives. Aldine, Hawthorne, New York, pp 173-192 
Crockett CM, Bowers CL, Bowden DM, Sackett GP (1994) Sex differences in compatibility of pair-housed adult long tailed macaques. Am J Primatol 94:73-94

Crockford C, Wittig RM, Langergraber K, et al (2013) Urinary oxytocin and social bonding in related and unrelated wild chimpanzees. Proc R Soc B Biol Sci 280:20122765 . doi: 10.1098/rspb.2012.2765

Crockford C, Wittig RM, Whitten PL, et al (2008) Social stressors and coping mechanisms in wild female baboons (Papio hamadryas ursinus). Horm Behav 53:254-265 . doi: 10.1016/j.yhbeh.2007.10.007

Crofoot MC, Rubenstein DI, Maiya AS, Berger-Wolf TY (2011) Aggression, grooming and group-level cooperation in white-faced capuchins (Cebus capucinus): Insights from social networks. Am J Primatol 73:821-833 . doi: 10.1002/ajp.20959

Danby S, Thompson C, Theobald M, Thorpe K (2012) Children's strategies for making friends when starting school. Aust J Early Child 37:63-71

Davenport MD, Tiefenbacher S, Lutz CK, et al (2006) Analysis of endogenous cortisol concentrations in the hair of rhesus macaques. Gen Comp Endocrinol 147:255-261 . doi: 10.1016/j.ygcen.2006.01.005

de Ruiter J, van Hooff J, Scheffrahn W (1994) Social and genetic aspecs of paternity in wild long-tailed macaques (Macaca fascicularis). Behaviour 129:203-224 . doi: 10.1163/156853994X00613

de Ruiter JR, Geffen E (1998) Relatedness of matrilines, dispersing males and social groups in long-tailed macaques (Macaca fascicularis). Proc R Soc B Biol Sci 265:79-87 . doi: 10.1098/rspb.1998.0267

de Vries H (1998) Finding a dominance order most consitent with a linear hierarchy: a new procedure and review. Anim Behav 55:827-843 . doi: 10.1006/anbe.1997.0708

de Vries H (1995) An improved test of linearity in dominance hierarchies containing unknown or tied relationships. Anim Behav 50:1375-1389 . doi: 10.1016/0003-3472(95)80053-0

Dewsbury DA (1981) Effects of novelty of copulatory behavior: The Coolidge effect and related phenomena. Psychol Bull 89:464-482 . doi: 10.1037/0033-2909.89.3.464

Dittus WPJ (1975) Population dynamics of the toque monkey, Macaca sinica. In: Tuttle RH (ed) Socioecology and psychology of primates. Mouton, The Hague, pp 125-151

Doyle LA, Baker KC, Cox LD (2010) Physiological and behavioral effects of social introduction on adult male rhesus macaques. Am J Primatol 70:542-550 . doi: 10.1002/ajp.20526

Drickamer LC, Vessey SH (1973) Group changing in free-ranging male rhesus monkeys. Primates 14:359-368 . doi: 10.1007/BF01731357

Dubuc C, Brent LJN, Accamando AK, et al (2009) Sexual skin color contains information about the timing of the fertile phase in free-ranging Macaca mulatta. Int J Primatol 30:777-789 . doi: 10.1007/s10764-009-9369-7

Dunayer ES, Berman CM (2017) Infant handling enhances social bonds in free-ranging rhesus macaques (Macaca mulatta). Behaviour 154:875-907 . doi: 10.1163/1568539X-00003448

Dunbar RIM, Sharman M (1983) Female competition for access to males affects birth rate in baboons. Behav Eco Sociobiol 13:157-159

Ebensperger LA (1998) Strategies and counterstragies to infanticide in mammals. Biol Rev 73:321-346

Ehardt CL, Bernstein IS (1986) Matrilineal overthrows in rhesus monkey groups. Int J Primatol 7:157-181 . doi: $10.1007 / B F 02692316$

Engelhardt A, Heistermann M, Hodges JK, et al (2006) Determinants of male reproductive success in wild long-tailed macaques (Macaca fascicularis) - Male monopolisation, female mate choice or post-copulatory mechanisms? Behav Ecol Sociobiol 59:740-752 . doi: 10.1007/s00265-005-0104-x

Engh AL, Beehner JC, Bergman TJ, et al (2006) Female hierarchy instability, male immigration and infanticide increase glucocorticoid levels in female chacma baboons. Anim Behav 71:1227-1237 . doi: 10.1016/j. anbehav.2005.11.009

Evers E, de Vries H, Spruijt BM, Sterck EHM (2015) Emotional bookkeeping and high partner selectivity are necessary for the emergence of partner-specific reciprocal affiliation in an agent-based model of primate groups. PLoS One 10:e0118921. doi: 10.1371/journal.pone.0118921

Fairbanks LA, Jorgensen MJ, Bailey JN, et al (2011) Heritability and genetic correlation of hair cortisol in vervet monkeys in low and higher stress environments. Psychoneuroendocrinology 36:1201-1208 . doi: 10.1016/j. psyneuen.2011.02.013 
Fedigan LM (2003) Impact of male takeovers on infant deaths, births and conceptions in Cebus capucinus at Santa Rosa, Costa Rica. Int J Primatol 24:723-741 . doi: 10.1023/A:1024620620454

Fedigan LM, Jack KM (2004) The demographic and reproductive context of male replacements in Cebus capucinus. Behaviour 141:755-775

Fernández D (2017) Consequences of a male takeover on mating skew in wild Sanje mangabeys. Am J Primatol 79:1-13. doi: 10.1002/ajp.22532

Foerster S, Cords M, Monfort SL (2011) Social behavior, foraging strategies, and fecal glucocorticoids in female blue monkeys (Cercopithecus mitis): Potential fitness benefits of high rank in a forest guenon. Am J Primatol 73:870-882 doi: 10.1002/ajp.20955

Fragaszy DM, Baer J, Adams-Curtis L (1994) Introduction and integration of strangers into captive groups of tufted capuchins (Cebus apella). Int J Primatol 15:399-420

Fraser D (2008) Understanding animal welfare. Acta Vet Scand 50 Suppl 1:1-7 . doi: 10.1186/1751-0147-50-S1-S1

Freidin E, Carballo F, Bentosela M (2017) Direct reciprocity in animals: The roles of bonding and affective processes. Int J Psychol 52:163-170 . doi: 10.1002/ijop.12215

Fruteau C, Range F, Noë R (2010) Infanticide risk and infant defence in multi-male free-ranging sooty mangabeys, Cercocebus atys. Behav Processes 83:113-118 . doi: 10.1016/j.beproc.2009.11.004

Georgiev A V, Christie D, Rosenfield KA, et al (2016) Breaking the succession rule: the costs and benefits of an alpha-status take-over by an immigrant rhesus macaque on Cayo Santiago. Behaviour 153:325-351. doi: $10.1163 / 1568539 X-00003344$

Gerber L, Krützen M, de Ruiter JR, et al (2015) Postdispersal nepotism in male long-tailed macaques (Macaca fascicularis). Ecol Evol 6:46-55 . doi: 10.1002/ece3.1839

Gilby IC, Brent LJN, Wroblewski EE, et al (2013) Fitness benefits of coalitionary aggression in male chimpanzees. Behav Ecol Sociobiol 67:373-381 . doi: 10.1007/s00265-012-1457-6

Glander KE (1992) Dispersal patterns in Costa Rican mantled howling monkeys. Int J Primatol 13:415-436 . doi: 10.1007/BF02547826

Gould L (1996) Male-female affiliative relationships in naturally occurring ringtailed lemurs (Lemur catta) at the Beza-Mahafaly Reserve, Madagascar. Am J Primatol 39:63-78

Greenwood PJ (1980) Mating systems, philopatry and dispersal in birds and mammals. Anim Behav 28:1140-1162 . doi: 10.1016/s0003-3472(80)80103-5

Grimm D (2018) Record number of monkeys being used in U.S. research. Sciencemag.org

Gros-louis J, Perry S, Manson JH (2003) Violent coalitionary attacks and intraspecific killing in wild white-faced capuchin monkeys (Cebus capucinus). Primates 44:341-346 . doi: 10.1007/s10329-003-0050-z

Hanby JP, Brown CE (1974) The development of sociosexual behaviours in Japanese macaques (Macaca fuscata). Behaviour 49:152-195 . doi: 10.1177/003754977302100201

Hannibal DL, Bliss-moreau E, Vandeleest J (2017) Laboratory rhesus macaque social housing and social changes: implications for research. 79:1-14 . doi: 10.1002/ajp.22528

Harcourt AH (1987) Dominance and fertility among female primates. J Zool 213:471-487 . doi: 10.1111/j.14697998.1987.tb03721.x

Haunhorst CB, Heesen M, Ostner J, Schülke O (2017) Social bonds with males lower the costs of competition for wild female Assamese macaques. Anim Behav 125:51-60 . doi: 10.1016/j.anbehav.2017.01.008

Haunhorst CB, Schülke O, Ostner J (2016) Opposite-sex social bonding in wild Assamese macaques. Am J Primatol 78:872-882 . doi: 10.1002/ajp.22554

Hayakawa S, Soltis J (2011) Troop takeover and reproductive success of wild male Japanese macaques on Yakushima island (Macaca fuscata yakui). Int J Zool 2011: . doi: 10.1155/2011/308469

Henzi SP, Barrett L (1999) The value of grooming to female primates. Primates 40:47-59 . doi: 10.1007/BF02557701

Henzi SP, Lucas JW (1980) Observations on the inter-troop movement of adult vervet monkeys (Cercopithecus aethiops). Folia Primatol 33:220-235 
Hill DA (1990) Social relationships between adult male and female rhesus macaques: II. non-sexual affiliative behaviour. Primates 31:33-50 . doi: 10.1007/BF02381028

Hohmann G, Gerloff U, Tautz D, Fruth B (1999) Social bonds and genetic ties: Kinship, association and affiliation in a community of bonobos (Pan paniscus). Behaviour 136:1219-1235 . doi: 10.1163/ 156853999501739

Hollingsworth HL, Buysse V (2009) Establishing friendships in early childhood inclusive settings: What roles do parents and teachers play? J Early Interv 31:287-307 . doi: 10.1177/1053815109352659

Honess PE, Marin CM (2006) Behavioural and physiological aspects of stress and aggression in nonhuman primates Neurosci Biobehav Rev 30:390-412 . doi: 10.1016/j.neubiorev.2005.04.003

Hrdy SB (1977) Infanticide as a Primate Reproductive Strategy. Am Sci 65:40-49

Hrdy SB (1979) Infanticide among animals: A review, classification, and examination of the implications for the reproductive strategies of females. Ethol Sociobiol 1:13-40 . doi: 10.1016/0162-3095(79)90004-9

Hrdy SB (1999) The woman that never evolved, Revised ed. Harvard University Press, Cambridge, Massachusetts, and London, England

Huchard E, Cowlishaw G (2011) Female-female aggression around mating: An extra cost of sociality in a multimale primate society. Behav Ecol 22:1003-1011. doi: 10.1093/beheco/arr083

Ichino S (2005) Attacks on a wild infant ring-tailed lemur (Lemur catta) by immigrant males at Berenty, Madagascar: Interpreting infanticide by males. Am J Primatol 67:267-272 . doi: 10.1002/ajp.20183

Ihara Y, Collins DA, Oda R, Matsumoto-Oda A (2016) Testing socially mediated estrous synchrony or asynchrony in wild baboons. Behav Ecol Sociobiol 70:1921-1930 . doi: 10.1007/s00265-016-2198-8

Inoue E, Takenaka O (2007) The effect of male tenure and female mate choice on paternity in free-ranging Japanese macaques. Am J Primatol 70:62-68 . doi: 10.1002/ajp.20457

Isbell LA (1991) Contest and scramble competition: Patterns of female aggression and ranging behavior among primates. Behav Ecol 2:143-155 . doi: 10.1093/beheco/2.2.143

Isbell LA (1990) Sudden short-term increase in mortality of vervet monkeys (Cercopithecus aethiops) due to leopard predation in Amboseli National Park, Kenya. Am J Primatol 21:41-52 . doi: 10.1002/ajp.1350210105

Isbell LA, Cheney DL, Seyfarth RM (1993) Are immigrant vervet monkeys, Cercopithecus aethiops, at greater risk of mortality than residents? Anim Behav 45:729-734 . doi: 10.1006/ANBE.1993.1087

Isbell LA, van Vuren D (1996) Differential costs of locational and social dispersal and their consequences for female group-living primates. Behaviour 133:14-34

Jack KM, Fedigan L (2004) Male dispersal patterns in white-faced capuchins, Cebus capucinus. Part 2: Patterns and causes of secondary dispersal. Anim Behav 67:771-782 . doi: 10.1016/j.anbehav.2003.06.015

Janson C, Baldovino MC, Bitetti M Di (2012) The group life cycle and demography of brown capuchin monkeys (Cebus [apella] nigritus) in Iguazú National Park, Argentina. In: Kappeler PM, Watts DP (eds) Long-term field studies of primates. Springer, Berlin, Heidelberg, pp 185-212

Jones KC (1983) Inter-Troop transfer of lemur catta males at Berenty, Madagascar. Folia Primatol 40:145-160 . doi: $10.1159 / 000156096$

Kappeler P, Fichtel C (2011) Female reproductive competition in Eulemur rufifrons: eviction and reproductive restraint in a plurally breeding Malagasy primate. Mol Ecol 21:685-698 . doi: 10.1111/j.1365294X.2011.05255.x

Kawai M (1960) A field experiment on the process of group formation in the Japanese monkey (Macaca fuscata), and the releasing of the group at Ôhirayama. Primates 2:181-255 . doi: 10.1007/BF01659168

Kawazoe T, Sosa S (2019) Social networks predict immigration success in wild Japanese macaques. Primates 60:213-222 . doi: 10.1007/s10329-018-0702-7

Keane B, Dittus WPJ, Melnick DJ (1997) Paternity assessment in wild groups of toque macaques Macaca sinica at Polonnaruwa, Sri Lanka using molecular markers. Mol Ecol 6:267-282 . doi: 10.1046/j.1365-294X.1997.00178.x

Kempes MM, Gulickx MMC, van Daalen HJC, et al (2008) Social competence is reduced in socially deprived rhesus monkeys (Macaca mulatta). J Comp Psychol 122:62-67 . doi: 10.1037/0735-7036.122.1.62 
Kirschbaum C, Tietze A, Skoluda N, Dettenborn L (2009) Hair as a retrospective calendar of cortisol productionIncreased cortisol incorporation into hair in the third trimester of pregnancy. Psychoneuroendocrinology 34:32-37 . doi: 10.1016/j.psyneuen.2008.08.024

Koyama N, Nakamichi M, Ichino S, Takahata Y (2002) Population and social dynamics changes in ring-tailed lemur troops at Berenty, Madagascar between 1989- 1999. Primates 43:291-314

Koyama NF (2001) The long-term effects of reconciliation in Japanese macaques Macaca fuscata. Ethology 107:975987 . doi: 10.1046/j.1439-0310.2001.00731.x

Krause J, Ruxton G (2002) Living in Groups. Oxford University Press, New York

Kuester J, Paul A (1999) Male migration in Barbary macaques (Macaca sylvanus) at Affenberg Salem. Int J Primatol 20:85-106 . doi: 10.1023/A:1020536317646

Kulik L, Muniz L, Mundry R, Widdig A (2012) Patterns of interventions and the effect of coalitions and sociality on male fitness. Mol Ecol 21:699-714 . doi: 10.1111/j.1365-294X.2011.05250.x

Ladd GW (1990) Having friends, keeping friends, making friends, and being liked by peers in the classroom: Predictors of children's early school adjustment? Child Dev 61:1081-1100

Langergraber K, Mitani J, Vigilant L (2009) Kinship and social bonds in female chimpanzees (Pan troglodytes). Am J Primatol 71:840-851 . doi: 10.1002/ajp.20711

Langergraber KE, Mitani JC, Watts DP, Vigilant L (2013) Male-female socio-spatial relationships and reproduction in wild chimpanzees. Behav Ecol Sociobiol 67:861-873 . doi: 10.1007/s00265-013-1509-6

Larson SM, Ruiz-lambides A, Platt ML, Brent LJN (2018) Social network dynamics precede a mass eviction in groupliving rhesus macaques. Anim Behav 136:185-193 . doi: 10.1016/j.anbehav.2017.08.019

Lemasson A, Palombit RA, Jubin R (2008) Friendships between males and lactating females in a free-ranging group of olive baboons (Papio hamadryas anubis): Evidence from playback experiments. Behav Ecol Sociobiol 62:1027-1035 . doi: 10.1007/s00265-007-0530-z

Lewis RJ (2008) Social influences on group membership in Propithecus verreauxi verreauxi. Int J Primatol 29:12491270 . doi: 10.1007/s10764-008-9304-3

Lewis RJ, Razafindrasamba SM, Tolojanahary JP (2003) Observed infanticide in a seasonal breeding prosimian (Propithecus verreauxi verreauxi) in Kirindy Forest, Madagascar. Folia Primatol 74:101-103 . doi: 10.1159/000070006

Lindburg DG (1969) Rhesus monkeys: mating season mobility of adult males. Science (80- ) 166:1176-1178

Line SW, Morgan KN, Markowitz H, et al (1990) Behavioral responses of female long-tailed macaques (Macaca fascicularis) to pair formation. Lab Primate Newsl 29:1-8

Liu BJ, Wu CF, Garber PA, et al (2018) Effects of group size and rank on mother-infant relationships and reproductive success in rhesus macaques (Macaca mulatta). Am J Primatol 80:e22881 . doi: 10.1002/ajp.22881

Loy J (1971) Estrous behavior of free-ranging rhesus monkeys (Macaca mulatta). Primates 12:1-31 . doi: 10.1007/ BF01730379

Lukas D, Huchard E (2014) The evolution of infanticide by males in mammalian societies. Science (80- ) 346:841-844 . doi: 10.1126/science. 1257226

Lutz CK, Novak MA (2005) Primate natural history and social behavior: implications for laboratory housing. In: WolfeCoote S (ed) The laboratory primate. Elsevier Academic Press, London, pp 133-142

MacCormick HA, MacNulty DR, Bosacker AL, et al (2012) Male and female aggression: lessons from sex, rank, age, and injury in olive baboons. Behav Ecol 23:684-691 . doi: 10.1093/beheco/ars021

Mainguy J, Côté SD, Cardinal E, Houle M (2008) Mating tactics and mate choice in relation to age and social rank in male mountain goats. J Mammal 89:626-635 . doi: 10.1644/07-MAMM-A-234R.1

Makwana SC (1978) Field ecology and behaviour of the rhesus macaque (Macaca mulatta): I. Group composition, home range, roosting sites, and foraging routes in the Asarori Forest. Primates 19:483-492 . doi: 10.1007/ BF02373310

Mallapur A, Choudhury BC (2003) Behavioral abnormalities in captive nonhuman primates. J Appl Anim Welf Sci 6:275-284 . doi: 10.1207/s15327604jaws0604_2 
Manson JH (1995) Do female rhesus macaques choose novel males? Am J Primatol 37:285-296 . doi: 10.1002/ ajp. 1350370403

Manson JH (1994) Mating patterns, mate choice, and birth season heterosexual relationships in free-ranging rhesus macaques. Primates 35:417-433. doi: 10.1007/BF02381951

Marty PR, Hodges K, Agil M, Engelhardt A (2016) Determinants of immigration strategies in male crested macaques (Macaca nigra). Sci Rep 6:32028 . doi: 10.1038/srep32028

Marty PR, Hodges K, Agil M, Engelhardt A (2017a) Alpha male replacements and delayed dispersal in crested macaques (Macaca nigra). Am J Primatol 79:e22448 . doi: 10.1002/ajp.22448

Marty PR, Hodges K, Heistermann M, et al (2017b) Is social dispersal stressful? A study in male crested macaques (Macaca nigra). Horm Behav 87:62-68 . doi: 10.1016/j.yhbeh.2016.10.018

Massen JJM, Overduin-de Vries AM, de Vos-Rouweler AJM, et al (2012) Male mating tactics in captive rhesus macaques (Macaca mulatta): The influence of dominance, markets, and relationship quality. Int J Primato 33:73-92 . doi: 10.1007/s10764-011-9552-5

Massen JJM, Sterck EHM (2013) Stability and durability of intra- and intersex social bonds of captive rhesus macaques (Macaca mulatta). Int J Primatol 34:770-791 . doi: 10.1007/s10764-013-9695-7

Massen JJM, Sterck EHM, De Vos H (2010) Close social associations in animals and humans: functions and mechanisms of friendship. Behaviour 147:1379-1412 . doi: 10.1163/000579510X528224

Matsumura S, Okamoto K (1998) Frequent harassment of mounting after a takeover of a group of moor macaques (Macaca maurus). Primates 39:225-230 . doi: 10.1007/BF02557734

McCowan B, Beisner B, Hannibal D (2018) Social management of laboratory rhesus macaques housed in large groups using a network approach: a review. Behav Processes 156:77-82 . doi: 10.1016/j.beproc.2017.11.014

McFadden D (1973) Conditional logit analysis of qualitative choice behavior. In: Zarembka P (ed) Frontiers in Econometrics (Economic theory and mathematical economics). Academic Press, pp 105-142

Mcfarland R, Murphy D, Lusseau D, et al (2017) The "strength of weak ties" among female baboons: fitness-related benefits of social bonds. Anim Behav 126:101-106 . doi: 10.1016/j.anbehav.2017.02.002

Meder A (1990) Integration of hand-reared gorillas into breeding groups. Zoo Biol 9:157-164 . doi: 10.1002/ zoo.1430090210

Mehlman P, T., Higley JD, et al (1995) Correlation of CSF 5-HIAA concentration with sociality and the timing of emigration in free-ranging primates. Am J Psychiatry 152:907-913 . doi: 10.1176/ajp.152.6.907

Mehlman PT (1986) Male intergroup mobility in a wild population of the Barbary macaque (Macaca sylvanus), Ghomaran rif mountains, Morocco. Am J Primatol 10:67-81 . doi: 10.1002/ajp.1350100107

Ménard N, Vallet D (1993) Dynamics of fission in a wild Barbary macaque group (Macaca sylvanus). Int J Primatol 14:479-500 . doi: 10.1007/BF02192778

Michael RP, Wilson M (1973) Changes in the sexual behaviour of male rhesus monkeys (M. mulatta) at puberty: Comparisons with the behaviour of adults. Folia Primatol 19:384-403 . doi: 10.1159/000155554

Mineka S, Suomi SJ (1978) Social separation in monkeys. Psychol Bull 85:1376-1400

Mitani JC (2009) Male chimpanzees form enduring and equitable social bonds. Anim Behav 77:663-640

Mittermeier RA, Wilson DE, Rylands AB (eds) (2013) Handbook of mammals of the world: primates. Lynx Edicions

Moore J, Ali R (1984) Are dispersal and inbreeding avoidance related? Anim Behav 32:94-112 . doi: 10.1016/S00033472(84)80328-0

Muniz L, Perry S, Manson JH, et al (2010) Male dominance and reproductive success in wild white-faced capuchins (Cebus capucinus) at Lomas Barbudal, Costa Rica. Am J Primatol 72:1118-1130 . doi: 10.1002/ajp.20876

Neville MK (1968a) A free-ranging rhesus monkey troop lacking adult males. J Mammal 49:711-733

Neville MK (1968b) Male leadership change in a free-ranging troop of Indian rhesus monkeys (Macaca mulatta). Primates 9:13-27 . doi: 10.1007/BF01795883

O’Brien TG (1991) Female-male social interactions in wedge-capped capuchin monkeys: benefits and costs of group living. Anim Behav 41:555-567 . doi: 10.1016/S0003-3472(05)80896-6 
Oates-O'Brien RS, Farver TB, Anderson-Vicino KC, et al (2010) Predictors of matrilineal overthrows in large captive breeding groups of rhesus macaques (Macaca mulatta). J Am Assoc Lab Anim Sci 49:196-201

Olsson IAS, Westlund K (2007) More than numbers matter: The effect of social factors on behaviour and welfare of laboratory rodents and non-human primates. Appl Anim Behav Sci 103:229-254 . doi: 10.1016/j. applanim.2006.05.022

Olupot W, Waser PM (2001) Correlates of intergroup transfer in male grey-cheeked mangabeys. Int J Primatol 22:169-187

Olupot W, Waser PM (2005) Patterns of male residency and intergroup transfer in gray-cheeked mangabeys (Lophocebus albigena). Am J Primatol 349:331-349 . doi: 10.1002/ajp.20160

Ostner J, Schülke O (2014) The evolution of social bonds in primate males. Behaviour 151:871-906 . doi $10.1163 / 1568539 X-00003191$

Ostner J, Vigilant L, Bhagavatula J, et al (2013) Stable heterosexual associations in a promiscuous primate. Anim Behav 86:623-631 . doi: 10.1016/j.anbehav.2013.07.004

Overduin-De Vries AM, Massen JJM, Spruijt BM, Sterck EHM (2012) Sneaky monkeys: An audience effect of male rhesus macaques (Macaca mulatta) on sexual behavior. Am J Primatol 74:217-228 . doi: 10.1002/ajp.21988

Overduin-de Vries AM, Olesen CU, de Vries H, et al (2013) Sneak copulations in long-tailed macaques (Macaca fascicularis): no evidence for tactical deception. Behav Ecol Sociobiol 67:101-111 . doi: 10.1007/s00265-012$1430-4$

Packer C (1979) Inter-troop transfer and inbreeding avoidance in Papio anubis. Anim Behav 27:1-36 . doi: 10.1016/0003-3472(79)90126-X

Packer C, Pusey AE (1979) Female aggression and male membership in troops of Japanese macaques and olive baboons. Folia Primatol 31:212-218 . doi: 10.1159/000155884

Palombit RA (2012) Infanticide: male strategies and female counterstrategies. In: Mitani JC, Call J, Kappeler PM, et al. (eds) The evolution of primate societies. University of Chicago Press, Chicago and London, pp 432-468

Palombit RA (2009) "Friendship" with males: A female counterstrategy to infanticide in chacma baboons of the Okavango delta. Sex Coerc Primates Humans An Evol Perspect Male Aggress Against Females 54:599-614

Palombit RA, Cheney DL, Seyfarth RM (2001) Female-female competition for male "friends" in wild chacma baboons, Papio cynocephalus ursinus. Anim Behav 61:1159-1171 . doi: 10.1006/anbe.2000.1690

Palombit RA, Seyfarth RM, Cheney DL (1997) The adaptive value of "friendships" to female baboons: Experimental and observational evidence. Anim Behav 54:599-614 . doi: 10.1006/anbe.1996.0457

Parga JA (2010) Evaluation of male inter-troop transfer as a mating strategy among ring-tailed lemurs on St. Catherines Island, USA. Folia Primatol 81:146-162 . doi: 10.1159/000316644

Pereira ME (1983) Abortion following the immigration of an adult male baboon (Papio cynocephalus). Am J Primato 4:93-98 . doi: 10.1002/ajp.1350040109

Pereira ME, Weiss ML (1991) Female mate choice, male migration, and the threat of infanticide in ringtailed lemurs Behav Ecol Sociobiol 28:141-152 . doi: 10.1007/BF00180991

Perry S (1998) A case report of a male rank reversal in a group of wild white-faced capuchins (Cebus capucinus). Primates 39:51-70

Plavcan JM (2001) Sexual dimorphism in primate evolution. Yearb Phys Anthropol 44:25-53 . doi: 10.1002/ ajpa. 10011

Plavcan JM, van Schaik CP (1997) Intrasexual competition and body weight dimorphism in anthropoid primates. Am J Phys Anthropol 103:37-68 . doi: 10.1002/ajpa.1330870407

Prescott MJ, Nixon ME, Farningham DAH, Naiken S (2012) Laboratory macaques: When to wean? Appl Anim Behav Sci 137:194-207 . doi: 10.1016/j.applanim.2011.11.001

Pryce CR, Rüedi-Bettschen D, Dettling AC, Feldon J (2002) Early life stress: Long-term physiological impact in rodents and primates. Physiology 17:150-155 . doi: 10.1152/nips.01367.2001

Pusey AE (1987) Sex-biased dispersal and inbreeding avoidance in birds and mammals. Trends Ecol Evol 2:295-299 doi: 10.1016/0169-5347(87)90081-4 
Pusey AE (2012) Magnitude and sources of variation in female reproductive performance. In: Mitani JC, Call J, Kappeler PM, et al. (eds) The Evolution of Primate Societies. The University of Chicago Press, Chicago and London, pp 343-366

Qi XG, Li BG, Garber PA, et al (2009) Social dynamics of the golden snub-nosed monkey (Rhinopithecus roxellana): Female transfer and one-male unit succession. Am J Primatol 71:670-679 . doi: 10.1002/ajp.20702

Ragen BJ, Bales KL (2013) Oxytocin and vasopressin in non-human primates. In: Choleris E, Pfaff DW, Kavaliers M (eds) Oxytocin, vasopressin and related peptides in the regulation of behavior. Cambridge University Press, Cambridge, pp 288-109

Raleigh MJ, McGuire MT (1989) Female influences on male dominance acquisition in captive vervet monkeys, Cercopithecus aethiops sabaeus. Anim Behav 38:59-67 . doi: 10.1016/S0003-3472(89)80065-X

Ramírez-Llorens P, Di Bitetti MS, Baldovino MC, Janson CH (2008) Infanticide in black capuchin monkeys (Cebus apella nigritus) in Iguazú National Park, Argentina. Am J Primatol 70:473-484 . doi: 10.1002/ajp.20522

Rebout N, Thierry B, Sanna A, et al (2017) Female mate choice and male-male competition in Tonkean macaques: Who decides? Ethology 123:365-375 . doi: 10.1111/eth.12605

Regan HJO, Kitchener AC (2005) The effects of captivity on the morphology of captive, domesticated and fera mammals. 35:215-230

Reinhardt V, Liss C, Stevens C (1995) Social housing of previously single-housed macaques: what are the options and the risks? Anim Welf 4:307-328

Rennie AE, Buchanan-Smith HM (2006) Refinement of the use of non-human primates in scientific research. Part II: Housing, husbandry and acquisition. Anim Welf 15:215-238

Richard AF, Rakotomanga P, Schwartz M (1993) Dispersal by Propithecus verreauxi at Beza Mahafaly, Madagaskar: 1984-1991. Am J Primatol 30:1-20

Roberts EK, Lu A, Bergman TJ, Beehner JC (2012) A bruce effect in wild geladas. Science (80- ) 335:1222-1226 . doi: $10.1126 /$ science. 1213600

Rodriguez-Llanes JM, Verbeke G, Finlayson C (2009) Reproductive benefits of high social status in male macaques (Macaca). Anim Behav 78:643-649 . doi: 10.1016/j.anbehav.2009.06.012

Rose RM, Gordon TP, Bernstein IS (1972) Plasma testosterone levels in the male rhesus: influences of sexual and social stimuli. Science (80- ) 178:643-645

Rowe N, Myers M (eds) (2016) All the Worlds Primates. Pogonias Press, Charlestown, Rhode Island

Rowell TE (1974) Contrasting adult male roles in different species of nonhuman primates. Arch Sex Behav 3:143-149 doi: 10.1007/BF01540998

Rox A, de Vries H, Louwerse AL, Sterck EHM (2018) Female social behaviour during three male introductions in captive groups of rhesus macaques. Appl Anim Behav Sci 207:89-97 . doi: 10.1016/j.applanim.2018.07.006

Rox A, van Vliet AH, Sterck EHM, et al (2019) Factors determining male introduction success and long-term stability in captive rhesus macaques. PLoS One 14:e0219972

Russel WM., Burch RL (eds) (1959) The principles of humane experimental technique. Methuen \& Cp. Ltd., London

Rutte C, Taborsky M, Brinkhof MWG (2006) What sets the odds of winning and losing? Trends Ecol Evol 21:16-21. doi: 10.1016/j.tree.2005.10.014

Saj TL, Sicotte P (2005) Male takeover in Colobus vellerosus at Boabeng-Fiema Monkey Sanctuary, central Ghana. Primates 46:211-214 . doi: 10.1007/s10329-004-0118-4

Samuels A, Altmann J (1986) Immigration of a Papio anubis male into a group of Papio cynocephalus baboons and. Int J Primatol 7:131-138 . doi: 10.1007/BF02692314

Sapolsky RM (1992) Cortisol concentrations and the social significance of rank instability among wild baboons. Psychoneuroendocrinology 17:701-709 . doi: 10.1016/0306-4530(92)90029-7

Sapolsky RM (1983) Endocrine aspects of social instability in the olive baboon (Papio anubis). Am J Primatol 5:365-379 . doi: 10.1002/ajp.1350050406

Schülke O, Bhagavatula J, Vigilant L, Ostner J (2010) Social bonds enhance reproductive success in male macaques. Curr Biol 20:2207-2210 . doi: 10.1016/j.cub.2010.10.058 
Setchell JM (2005) Do female mandrills prefer brightly colored males? Int J Primatol 26:715-735

Setchell JM, Huchard E (2010) The hidden benefits of sex: Evidence for MHC-associated mate choice in primate societies. BioEssays 32:940-948 . doi: 10.1002/bies.201000066

Setchell JM, Knapp LA, Wickings EJ (2006) Violent coalitionary attack by female mandrills against an injured alpha male. Am J Primatol 68:411-418 . doi: 10.1002/ajp

Setchell JM, Smith T, Wickings EJ, Knapp LA (2008) Factors affecting fecal glucocorticoid levels in semi-free-ranging female mandrills (Mandrillus sphinx). Am J Primatol 70:1023-1032 . doi: 10.1002/ajp.20594

Seyfarth RM, Cheney DL (2012) The evolutionary origins of friendship. Annu Rev Psychol 63:153-177 . doi: 10.1146/ annurev-psych-120710-100337

Shively CA, Kaplan JR, Adams MR (1986) Effects of ovariectomy, social instability and social status on female Macaca fascicularis social behavior. Physiol Behav 36:1147-1153 . doi: 10.1016/0031-9384(86)90492-0

Sicotte P, Teichroeb JA, Vayro J V., et al (2017) The influence of male takeovers on female dispersal in Colobus vellerosus. Am J Primatol 79:e22436 . doi: 10.1002/ajp.22436

Silk J (2002a) Using the ' $F$ '-word in primatology. Behaviour 139:421-446 . doi: 10.1163/156853902760102735

Silk JB (2002b) Kin selection in primate groups. Int J Primatol 23:849-875 . doi: 10.1023/A:1015581016205

Silk JB, Alberts SC, Altmann J (2003) Social bonds of female baboons enhance infant survival. Science (80- ) 302:1231-1234

Silk JB, Altmann J, Alberts SC (2006) Social relationships among adult female baboons (Papio cynocephalus) I. Variation in the strength of social bonds. Behav Ecol Sociobiol 61:183-195 . doi: 10.1007/s00265-006-0249-2

Silk JB, Beehner JC, Bergman TJ, et al (2009) The benefits of social capital: close social bonds among female baboons enhance offspring survival. Proc R Soc B Biol Sci 276:3099-3104 . doi: 10.1098/rspb.2009.0681

Silk JB, Beehner JC, Bergman TJ, et al (2010a) Female chacma baboons form strong, equitable, and enduring social bonds. Behav Ecol Sociobiol 64:1733-1747 . doi: 10.1007/s00265-010-0986-0

Silk JB, Beehner JC, Bergman TJ, et al (2010b) Strong and consistent social bonds enhance the longevity of female baboons. Curr Biol 20:1359-1361 . doi: 10.1016/j.cub.2010.05.067

Singh M, Kumara HN, Kumar MA, et al (2006) Male influx, infanticide, and female transfer in Macaca radiata radiata. Int J Primatol 27:515-528 . doi: 10.1007/s10764-006-9031-6

Smith JE, Powning KS, Dawes SE, et al (2011) Greetings promote cooperation and reinforce social bonds among spotted hyaenas. Anim Behav 81:401-415 . doi: 10.1016/j.anbehav.2010.11.007

Smuts BB, Smuts RW (1993) Male aggression and sexual coercion of females in nonhuman primates and Ooher mammals: evidence and theoretical implications. Adv Study Behav 22:1-63

Soltis J, Mitsunaga F, Shimizu K, et al (1997) Sexual selection in Japanese macaques I: female mate choice or male sexual coercion? Anim Behav 54:725-736 . doi: 10.1006/anbe.1997.0567

Soltis J, Thomsen R, Matsubayashi K, Takenaka O (2000) Infanticide by resident males and female counter-strategies in wild Japanese macaques (Macaca fuscata). Behav Ecol Sociobiol 48:195-202 . doi: 10.1007/s002650000224

Soltis J, Thomsen R, Takenaka O (2001) The interaction of male and female reproductive strategies and paternity in wild Japanese macaques, Macaca fuscata. Anim Behav 62:485-494 . doi: 10.1006/anbe.2001.1774

Sonnweber RS, Massen JJM, Fitch WT (2015) Post-copulatory grooming: a conditional mating strategy? Behav Ecol Sociobiol 69:1749-1759 . doi: 10.1007/s00265-015-1987-9

Sprague DS (1992) Life history and male intertroop mobility among Japanese Macaques. Int J Primatol 13:437-454

Sprague DS, Suzuki S, Takahashi H, Sato S (1998) Male life history in natural populations of Japanese macaques: Migration, dominance rank, and troop participation of males in two habitats. Primates 39:351-363 . doi: 10.1007/BF02573083

Städele V, Roberts ER, Barrett BJ, et al (2019) Male-female relationships in olive baboons (Papio anubis): Parenting or mating effort? J Hum Evol 127:81-92 . doi: 10.1016/j.jhevol.2018.09.003

Sterck E, Bontrop R, de Groot N, et al (2017) No postcopulatory selection against MHC-homozygous offspring: Evidence from a pedigreed captive rhesus macaque colony. Mol Ecol 26:3785-3793 . doi: 10.1111/mec.14153 
Sterck EHM, Korstjens AH (2000) Female dispersal and infanticide avoidance in primates. In: van Schaik CP, Janson $\mathrm{CH}$ (eds) Infanticide by males and its implications. Cambridge University Press, Cambridge, pp 293-321

Sterck EHM, Watts DP, vanSchaik CP (1997) The evolution of female social relationships in nonhuman primates. Behav Ecol Sociobiol 41:291-309 . doi: 10.1007/s002650050390

Sterck EHM, Willems EP, Van Hooff JARAM, Wich SA (2005) Female dispersal, inbreeding avoidance and mate choice in Thomas langurs (Presbytis thomasi). Behaviour 142:845-868 . doi: 10.1163/1568539055010093

Sugiyama Y, Ohsawa H (1975) Life history of male Japanese macaques at Ryozenyama.pdf. In: Kondo S, Kawai M, Ehara A (eds) Contemporary Primatology. Karger, Basel, pp 407-410

Suomi SJ (2005) Mother-infant attachment, peer relationships, and the development of social networks in rhesus monkeys. Hum Dev 48:67-79 . doi: 10.1159/000083216

Sussman RW (1992) Male life history and intergroup mobility among ringtailed lemurs (Lemur catta). Int J Primatol 13:395-413 . doi: 10.1007/BF02547825

Suzuki S, Hill DA, Sprague DS (1998) Intertroop transfer and dominance rank structure of nonnatal male Japanese macaques in Yakushima, Japan. Int J Primatol 19:703-722 . doi: 10.1023/A:1020329010009

Taylor SE (2010) Mechanisms linking early life stress to adult health outcomes. Proc Natl Acad Sci U S A 107:85078512 . doi: 10.1073/pnas.1003890107

Teichroeb JA, Jack KM (2017) Alpha male replacements in nonhuman primates: Variability in processes, outcomes, and terminology. Am J Primatol 79:e22674 . doi: 10.1002/ajp.22674

Teichroeb JA, Sicotte P (2008) Infanticide in ursine colobus monkeys (Colobus vellerosus) in Ghana: New cases and a test of the existing hypotheses. Behaviour 145:727-755 . doi: 10.1163/156853908783929160

Teichroeb JA, Wikberg EC, Sicotte P (2011) Dispersal in male ursine colobus monkeys (Colobus vellerosus): Influence of age, rank and contact with other groups on dispersal decisions. Behaviour 148:765-793 . doi: $10.1163 / 000579511 \times 577157$

Thierry B (2000) Covariation of conflict management patterns across macaque species. Nat Confl Resolut 106-128 doi: 10.1006/anbe.2001.1656

Thierry B (1990) Feedback loop between kinship and dominance: the macaque model. J Theor Biol 145:511-522. doi: 10.1016/S0022-5193(05)80485-0

Thierry B, Iwaniuk AN, Pellis SM (2000) The influence of phylogeny on the social behaviour of macaques (Primates: Cercopithecidae, genus Macaca). Ethology 106:713-728

Timmermans PJ, Schouten WG, Krijnen JC (1981) Reproduction of cynomolgus monkeys (Macaca fascicularis) in harems. Lab Anim 15:119-123

Trivers RL (1972) Parental investment and sexual selection. In: Campbell B (ed) Sexual selection and the descent of man. Aldine, Chicago, pp 136-179

Truelove MA, Martin AL, Perlman JE, et al (2017) Pair housing of Macaques: A review of partner selection, introduction techniques, monitoring for compatibility, and methods for long-term maintenance of pairs. Am J Primatol 79:1-15 . doi: 10.1002/ajp.22485

van Belle S, Kulp AE, Thiessen-Bock R, et al (2010) Observed infanticides following a male immigration event in black howler monkeys, Alouatta pigra, at Palenque National Park, Mexico. Primates 51:279-284 . doi: 10.1007/ s10329-010-0207-5

van Noordwijk MA, van Schaik CP (1985) Male migration and rank acquisition in wild long-tailed macaques (Macaca fascicularis). Anim Behav 33:849-861 . doi: 10.1016/S0003-3472(85)80019-1

van Noordwijk MA, van Schaik CP (2000) Career moves: transfer and rank challenge decisions by male long-tailed macaques. Behaviour 138:359-395 . doi: 10.1163/15685390152032505

van Noordwijk MA, van Schaik CP (1999) The effects of dominance rank and group size on remale lifetime reproductive success in wild long-tailed macaques, Macaca fascicularis. Primates 40:105-130

van Schaik CP, Janson CH (2000) Infanticide by males and its implications. Cambridge University Press, New York

van Schaik CP, Pandit SA, Vogel ER (2004) A model for within-group coalitionary aggression among males. Behav Ecol Sociobiol 57:101-109 . doi: 10.1007/s00265-004-0818-1 
Veenema $\mathrm{AH}$ (2009) Early life stress, the development of aggression and neuroendocrine and neurobiological correlates: What can we learn from animal models? Front Neuroendocrinol 30:497-518 . doi: 10.1016/j. yfrne.2009.03.003

Vernes MK, Louwerse AL (2010) BPRC's enrichment manual for macaques and marmosets. Biomedical Primate Research Centre, Rijswijk

Vessey SH (1971) Free-ranging rhesus monkeys: behavioural effects of removal, separation and reintroduction of group members. Behaviour 40:216-227

Wentzel KR, Erdley CA (1993) Strategies for making friends: Relations to social behavior and peer acceptance in early adolescence. Dev Psychol 29:819-826

Wheatley BP (1982) Adult male replacement in Macaca fascicularis of East Kalimantan, Indonesia. Int J Primatol $3: 203-219$

Whitten PL (1983) Diet and dominance among female vervet monkeys (Cercopithecus aethiops). Am J Primatol 5:139-159 . doi: 10.1002/ajp.1350050205

Widdig A, Streich WJ, Nürnberg P, et al (2006) Paternal kin bias in the agonistic interventions of adult female rhesus macaques (Macaca mulatta). Behav Ecol Sociobiol 61:205-214 . doi: 10.1007/s00265-006-0251-8

Wilson ME, Gordon TP (1979) Sexual activity of male rhesus monkeys introduced into a heterosexual group. Am J Phys Anthropol 50:515-523

Winkler P, Loch H, Vogel C (1984) Life history of Hanuman langurs (Presbytis entellus). Reproductive parameters, infant mortality, and troop development. Folia Primatol 43:1-23

Winston LA (1985) Social interactions among female rhesus macaques (Macaca mulatta) during the nonbreeding season: Responses to the introduction of males. Am J Primatol 9:243-257 . doi: 10.1002/ajp.1350090402

Wittig RM, Crockford C, Lehmann J, et al (2008) Focused grooming networks and stress alleviation in wild female baboons. Horm Behav 54:170-177 . doi: 10.1016/j.yhbeh.2008.02.009

Worlein JM, Kroeker R, Lee GH, et al (2017) Socialization in pigtailed macaques (Macaca nemestrina). Am J Primato 79:1-12 . doi: 10.1002/ajp.22556

Xia D-P, Li J-H, Garber PA, et al (2013) Grooming reciprocity in male Tibetan macaques. Am J Primatol 75:1009-1020 doi: 10.1002/ajp.22165

Yamada M (1963) A study of blood-relationship in the natural society of the Japanese macaque. Primates 4:43-65 . doi: 10.1007/BF01733671

Yamada M (1971) Five natural troops of Japanese monkeys on Shodoshima Island: II. A comparison of socia structure. Primates 12:125-150 . doi: 10.1007/BF01730391

Yamanashi Y, Morimura N, Mori Y, et al (2013) Cortisol analysis of hair of captive chimpanzees (Pan troglodytes). Gen Comp Endocrinol 194:55-63 . doi: 10.1016/j.ygcen.2013.08.013

Young C, Majolo B, Schülke O, Ostner J (2014) Male social bonds and rank predict supporter selection in cooperative aggression in wild Barbary macaques. Anim Behav 95:23-32 . doi: 10.1016/J.ANBEHAV.2014.06.007

Young KA, Liu Y, Wang Z (2008) The neurobiology of social attachment: A comparative approach to behavioral, neuroanatomical, and neurochemical studies. Comp Biochem Physiol- C Toxicol Pharmacol 148:401-410 . doi: 10.1016/j.cbpc.2008.02.004

Zaunmair P, Mangalam M, Kaumanns W, et al (2015) Patterns of dominance relationships among the females of a captive female-only group of lion-tailed macaques (Macaca silenus) during the course of the introduction of a new adult male. Curr Sci 109:803-807

Zhao QK (1994) Mating competition and intergroup transfer of males in Tibetan macaques (Macaca thibetana) at Mt. Emei, China. Primates 35:57-68 . doi: 10.1007/BF02381486 


\section{Nederlandse samenvatting}

Wanneer mensen of dieren hun bekende omgeving verruilen voor een onbekende, nieuwe omgeving spreekt men van migratie. Migratie is wijdverspreid in de dierenwereld en brengt vaak kosten en baten met zich mee. Als mensen en dieren niet alleen van fysieke omgeving veranderen, maar ook van sociale groep, komen er extra kosten bij voor het opbouwen van sociale relaties met nieuwe groepsgenoten. Deze sociale kosten van migratie zijn zeer relevant voor niet-humane primaten (verder primaten of apen genoemd), die vaak in sociale groepen met meerdere volwassen apenvrouwen en meerdere volwassen apenmannen leven. Vooral Oude Wereld apen leven vaak in dit soort groepen, waaruit mannen over het algemeen migreren terwijl vrouwen doorgaans hun hele leven in hun geboortegroep blijven. De mannelijke apen veranderen meerdere keren in hun leven van groep. Als zij een groep binnen komen, staan ze voor de moeilijke taak om een nieuwe sociale positie te veroveren tussen nauw verwante vrouwen die onderling een sterke band hebben, en meerdere mannen waarmee ze de competitie aan moeten gaan. Dat lukt niet altijd. Soms weet een nieuwe man geen plek in een groep te veroveren. Alleen met een goede sociale positie in een groep zal een man op de lange termijn kunnen overleven en zich kunnen voortplanten. Daardoor zijn de sociale kosten en baten van migratie niet alleen belangrijk om te bepalen of een man succesvol een nieuwe groep in kan komen, maar beïnvloeden ze ook de overleving en voortplanting, de zogenaamde fitness, van een man.

Onderzoekers die tot nu toe de sociale kosten en baten van migratie in apen hebben bestudeerd, concentreerden zich voornamelijk op de kosten van competitie tussen nieuwe en residente mannen in een groep. Maar niet enkel mannen ervaren kosten en baten tijdens migratie, ook de fitness van de vrouwen in de groep wordt beïnvloed door migratie van mannen. Nieuwe mannen zijn vaak agressief naar residente vrouwen en kunnen ze verwonden, of maken hun kinderen dood, ook wel infanticide genoemd. Vrouwen kunnen daarom agressief reageren op nieuwe mannen, of ze juist ontlopen. Maar vrouwen hebben ook vaak een seksuele voorkeur voor nieuwe mannen, door met ze te paren stimuleren ze mogelijk dat nieuwe mannen hun groep in komen. Ook kunnen vrouwen nieuwe mannen vlooien en vriendschappen met ze opbouwen, in ruil voor bescherming of seksuele toegang. Zulke vriendschappelijke relaties tussen mannen en vrouwen komen veelvuldig voor in apengroepen en leveren fitness-voordelen op voor hen allebei. Het gedrag van de vrouwen heeft zo invloed op de sociale kosten en baten die nieuwe mannen ervaren wanneer ze een nieuwe groep proberen in te komen. De rol van vrouwen tijdens migratie van mannen heeft echter nog maar weinig aandacht gekregen van onderzoekers en is niet systematisch bestudeerd. Het is van groot belang om ook de rol van alle nieuwe groepsgenoten van migrerende mannen in kaart te brengen, want alleen dan kunnen we begrijpen wat de kosten, baten en fitness consequenties van migratie in niet-humane primaten zijn.

Het is echter lastig om bij wilde apen systematisch onderzoek te doen naar het gedrag van vrouwelijke apen wanneer er een nieuwe man de groep in komt. Het is namelijk zeer variabel en niet te voorspellen wanneer er een nieuwe man een groep binnen zal komen, dat gebeurt soms een aantal keer per jaar en soms een aantal jaren 
niet. Dit proefschrift richt zich daarom op een vergelijkbaar proces in gevangenschap, met als doel de reactie van vrouwen op een nieuwe man in de groep beter te kunnen begrijpen. Daarvoor zijn introducties van nieuwe mannen in bestaande natuurlijke sociale groepen van resusapen (Macaca mulatta) of Java-apen ( $M$. fascicularis) bestudeerd bij het Biomedical Primate Research Centre (BPRC), in Rijswijk, Nederland. De mannen werden geïntroduceerd om inteelt te voorkomen, terwijl de sociale structuur van de groep verwante vrouwen bewaard blijft, vergelijkbaar met het wild. Tijdens deze introducties werden eenmansgroepen gevormd. Dit gaf ons de kans om verder te kijken dan de competitie tussen mannen en in te zoomen op het gedrag van de vrouwen. Daarmee werd nieuwe fundamentele kennis opgedaan over de effecten van het gedrag van vrouwen op fitness van migrerende apenmannen. Daarnaast is nieuwe toegepaste kennis vergaard over hoe introducties van nieuwe mannen het beste gemanaged kunnen worden. Introducties van nieuwe mannen zijn namelijk risicovol voor zowel nieuwe als residente dieren; nieuwe mannen kunnen residente vrouwen verwonden of infanticide plegen. Andersom kunnen vrouwen ook nieuwe mannen verwonden of zelfs doden, en kunnen ze voorkomen dat een nieuwe man zich weet te vestigen in hun groep. Daarom worden de introducties vanuit zowel een toegepast (Hoofdstuk 3-5) als een fundamenteel (Hoofdstuk 2 en 6-8) oogpunt bestudeerd.

Om te beginnen wordt in Hoofdstuk $\mathbf{2}$ in een literatuurreview van de bestaande informatie over interacties tussen vrouwen en nieuwe mannen besproken. Uit bestaande studies, die zich doorgaans op het gedrag van nieuwe en eventueel residente mannen richten, komt het beeld naar voren dat vrouwen regelmatig agressie vertonen richting nieuwe mannen. Deze agressie kan hoge kosten voor nieuwe mannen met zich mee kan brengen. Daarentegen is zeer weinig informatie over positief sociale interacties tussen vrouwen en nieuwe mannen, zoals vlooien en paren, beschikbaar. Bovendien is er weinig bekend over de fitness consequenties van interacties tussen residente vrouwen en nieuwe mannen. Deze hiaten in de huidige kennis vormen de basis voor dit proefschrift.

Het opvolgende deel van het proefschrift (Hoofdstuk 3-5) bekijkt de introducties van nieuwe mannen vanuit een toegepast perspectief. Daarbij is het doel om dierenwelzijn te verhogen door het optimaliseren van de introductieprocedure. De eerste stap is het beschrijven van de huidige introductieprocedure van het BPRC in Hoofdstuk 3. Dit hoofdstuk bevat een gedetailleerde omschrijving van de naturalistische huisvesting van de makaken (resusapen en Java-apen) bij het BPRC, waar de dieren in groepen leven waarin de natuurlijke sociale processen zo goed mogelijk worden nagebootst. Net als bij wilde makaken, blijven vrouwtjes gedurende hun hele leven in hun geboortegroep. Mannen blijven in hun geboortegroep tot aan de puberteit, terwijl volwassen mannen van groep wisselen. Bij het BPRC wisselt een man iedere 4 tot 5 jaar van groep, om inteelt met zijn dochters te vermijden. Deze manier van huisvesten is uniek voor onderzoeksinstituten. Daarnaast wordt besproken hoe de introductieprocedure van nieuwe mannen verloopt en welke afwegingen en keuzes er tijdens de 
introducties gemaakt worden. Deze informatie kan andere onderzoeksinstituten en locaties waar apen gehuisvest worden helpen om hun huisvestingsysteem aan te passen en zo dierenwelzijn te verbeteren.

In Hoofdstuk 4 wordt het algemene gedragspatroon van vrouwen beschreven gedurende drie verschillende introducties van een nieuwe man in een groep resusapen. We laten zien dat de leeftijd van een vrouw bepaalt of zij een nieuwe man in haar groep tolereert; oude vrouwen tolereren nieuwe mannen sneller dan jongere vrouwen. Daarnaast laten we zien dat vrouwen aan het begin van de introductie veel agressie en submissie vertonen naar hun nieuwe man, en veel met hem paren. Deze gedragingen nemen af naarmate de introductie vordert en de man beter integreert in de groep. Affiliatie van de vrouwen naar de nieuwe man neemt juist toe met de tijd. De veranderingen in sociaal gedrag waren vergelijkbaar tussen de drie introducties, wat impliceert dat er een algemeen gedragspatroon is tijdens introducties. Hoe snel gedrag afneemt en hoeveel van een bepaald gedrag wordt vertoond varieert wel tussen introducties. Deze observaties suggereren dat het veilig is om de man voltijds in de groep te laten wanneer vrouwen nog maar weinig submissief gedrag vertonen aan de man. Bovendien zou weinig agressie van vrouwen naar de man erop kunnen wijzen dat de man ook op de lange termijn een stabiele positie in de groep weet te behouden. Deze resultaten tonen aan hoe vrouwen op nieuwe mannen reageren en zijn een belangrijke stap naar het verder verbeteren van introductieprocedures bij apen in gevangenschap.

Hoofdstuk 5 bevat een evaluatie op basis van 64 introducties van resusaapmannen die bij het BPRC hebben plaatsgevonden tussen 2003 en 2018. Het onderzoek beschreven in dit hoofdstuk heeft als doel te bepalen hoe belangrijke aspecten, zoals de timing van de introducties, de eigenschappen van de nieuwe mannen, en de eigenschappen van de groep de uitkomst van introducties beïnvloeden. Daarbij zijn zowel het korte-termijn succes van de introducties als op de lange termijn de stabiliteit van de sociale positie van de man in zijn nieuwe groep bekeken. De data komen uit de database van het BPRC en bijbehorende introductieverslagen. De resultaten laten zien dat introducties succesvoller en stabieler zijn wanneer de introductieprocedure en de migratie patronen meer overeenkomst vertonen met de migratiepatronen van wilde makaken. Dat houdt in dat mannen pas geïntroduceerd zouden moeten worden wanneer ze ook in de natuur de dominantiepositie zouden overnemen; op de zogenaamde 'prime age', als ze op de top van hun fysieke conditie zijn. Daarnaast moet de groep maximaal drie families bevatten en moeten deze families meerdere volwassen vrouwen bevatten. Vrouwen moeten daarvoor in hun geboortegroep blijven, mannetjes zouden de groep pas moeten verlaten op de leeftijd dat ze dat in de natuur ook doen. Alleen dan leren ze genoeg sociale vaardigheden om een stabiele positie in hun nieuwe groep te behouden. Al met al laten deze resultaten zien dat het belangrijk is om makaken in zo natuurlijk mogelijke fokgroepen te huisvesten. Dit vermindert risico's bij introducties en resulteert in stabiele sociale groepen. Bovendien wordt aangenomen dat het welzijn van wilde dieren in gevangenschap het beste is wanneer zij natuurlijk gedrag kunnen vertonen. Het nabootsen van natuurlijke migratiepatronen stimuleert natuurlijk sociaal gedrag en zal het welzijn van de apen verhogen. 
Het laatste deel van dit proefschrift (Hoofdstuk 6-8) bekijkt de introducties van een fundamenteel wetenschappelijk oogpunt. Daarbij wordt voornamelijk gefocust op de baten die sociale interacties tussen vrouwen en hun nieuwe man opleveren. Hoofdstuk $\mathbf{6}$ beschrijft hoe goede sociale relaties tussen residente vrouwen en nieuwe mannen ontstaan. Het is bekend dat apen nauwe relaties met groepsgenoten vormen en dat deze relaties allerlei fitness-voordelen kunnen opleveren. Hoe deze sociale relaties ontstaan was echter onbekend. Het onderzoek beschreven in dit hoofdstuk richt zich op deze vraag en heeft als doel het mechanisme waarmee apen relaties opbouwen te verhelderen. Daarvoor zijn gedragsobservaties gedaan tijdens vier introducties van resusaapmannen in een sociale groep. De data laten zien dat de allereerste interacties tussen de vrouwen en de nieuwe man bepalend zijn voor de sociale relaties die na de introducties gevormd zijn. Vrouwen die hun nieuwe man al vroeg in de introductie tolereerden, namen vaker deel aan zowel agressieve, affiliatieve als seksuele interacties. Vooral vrouwen die affiliatie ontvingen van de man en veel met hem paarden tijdens de start van de introductie, bouwden uiteindelijk een goede relatie op met de nieuwe man. Van één introductie waren ook data beschikbaar van sociaal gedrag één jaar na de introductie. Deze data suggereren dat de relaties tussen de vrouwen en hun nieuwe man ook op de lange termijn standhouden. Al met al impliceert dit onderzoek dat de eerste interacties tussen vrouwen en een nieuwe man de fitness van beiden ook op de lange termijn kunnen beïnvloeden.

Hoofdstuk 7 focust op één van de andere voordelen die mannen kunnen ervaren wanneer ze een nieuwe groep binnenkomen; namelijk seksuele toegang tot vrouwen. Vrouwelijke apen hebben voorkeur voor het paren met bepaalde mannen, nieuwe mannen zijn vaak zeer geliefde paringspartners. Er wordt gedacht dat vrouwen niet in competitie met elkaar hoeven wanneer ze dezelfde man prefereren als paringspartner, een man kan immers met meerdere vrouwen paren. Dit is echter nog niet systematisch onderzocht. Tijdens de introducties bestudeerd in dit proefschrift, werden omstandigheden gecreëerd waaronder competitie tussen vrouwen verwacht kan worden; er is één man in de groep en die man is een zeer geliefde paringspartner. Daarom richt dit hoofdstuk zich op mogelijke strategieën die vrouwen gebruiken om seksuele toegang tot de nieuwe man te krijgen, op basis van data van vier introducties van resusaapmannen. De resultaten laten zien dat de vrouwen verschillende strategieën gebruikten om toegang te krijgen tot hun nieuwe man. Zowel vrouwen die de man vaker benaderden, als vrouwen die de man meer vlooiden en vrouwen die vaker agressie naar andere vrouwen vertoonden, paarden vaker met de nieuwe man dan vrouwtjes die zich passiever opstelden. Deze resultaten kunnen de traditionele kijk op apenmannen en-vrouwen veranderen, waarbij mannen worden gezien als veroveraars en vrouwen een ondergeschikte rol spelen. Het zijn niet alleen mannen die seksuele strategieën kunnen gebruiken en in competitie gaan om een paringspartner te veroveren. Ook vrouwen kunnen seksuele strategieën gebruiken, of ze dat ook daadwerkelijk doen hangt mogelijk af van de sociale context.

Het laatste onderzoek dat gepresenteerd wordt in dit proefschrift, in Hoofdstuk 8, focust op een factor die waarschijnlijk zeer bepalend is voor de reactie van vrouwen op 
een nieuwe man; het risico op infanticide. Het doden van een baby door een volwassen man komt bij veel apensoorten voor. Infanticide is duidelijk nadelig voor een moeder maar biedt een man een fitness-voordeel als hij niet de vader is van de gedode baby maar wel een volgend kind kan verwekken. Het risico op infanticide verschilt tussen apensoorten en tussen individuen van dezelfde soort. In dit hoofdstuk worden de eigenschappen van individuele vrouwen gekoppeld aan het risico op infanticide en hun stressreactie op een nieuwe man. Daarvoor zijn de niveaus van het stresshormoon cortisol bepaald in haar dat is verzameld na de introductie van een nieuwe man van zowel resusapen als Java-apen. Het cortisolniveau in dit haar is vervolgens vergeleken met het basiscortisolniveau uit haar dat is verzameld na een sociaal stabiele periode. Cortisol in haar reflecteert lange-termijn stressniveaus. De resultaten laten zien dat vrouwen die zogen (i.e. de vrouwen die direct risico lopen op infanticide) en vrouwen met een lager basiscortisolniveau de meeste stress ervaren tijdens de introductie van een nieuwe man. Bovendien waren de introducties meer stressvol voor Java-apen, de soort waarin infanticide door nieuwe mannen vaker voorkomt dan bij resusapen. Javaaap vrouwen kunnen deze stress bufferen door twee typen sociaal gedrag te vertonen dat het risico op infanticide verlaagt, enerzijds het ontlopen van de nieuwe man, of anderzijds juist vaak met hem paren. Dit suggereert dat infanticide risico inderdaad een belangrijke rol speelt tijdens introducties en dat het mogelijk de reactie van vrouwen op een nieuwe man beïnvloedt. Er was geen infanticide geobserveerd tijdens de bestudeerde introducties, maar enkel het risico op infanticide lijkt al voldoende om een stressrespons op te roepen. Deze stressreactie kan de fitness van vrouwen op de korte termijn verhogen, omdat de stress ze helpt om sneller te reageren op de gevaren die nieuwe mannen met zich meebrengen, zoals infanticide. Als de stress echter op de lange termijn aanhoudt, zoals in deze studie, is dit nadelig voor de gezondheid en voortplanting van vrouwen, en heeft dit negatieve fitness consequenties.

Ten slotte vat Hoofdstuk 9 de belangrijkste bevindingen van dit proefschrift samen en laat zien hoe de beschreven onderzoeken bijdragen aan het opvullen van de in hoofdstuk 2 geschetste hiaten in kennis. Zo vormt het gedrag van de vrouwen tijdens introducties van een nieuwe man de basis voor het verbeteren van het welzijn en het management van apengroepen in gevangenschap. Daarbij wordt kritisch gekeken naar de standaard huisvestingssystemen die biomedische onderzoekscentra vaak hanteren voor hun primaten. Daarnaast laten de studies uit dit proefschrift zien dat het sociale gedrag van de residente vrouwen een belangrijke rol speelt tijdens de migratie van mannen; vrouwen kunnen de kosten, de baten en de uitkomst van migratie beïnvloeden. Deze opgedane kennis over het gedrag van vrouwen draagt bij aan het oplossen van verschillende vraagstukken in zowel toegepast als fundamenteel onderzoek. Het onderzoek beschreven in dit proefschrift betreft een eerste stap richting het volledig begrijpen van de rol van alle groepsgenoten tijdens migratie van mannen. Daarnaast worden belangrijke overgebleven hiaten in kennis over het migratieproces van apen benoemd. Er moet met name meer informatie komen over de invloed van positief sociale interacties tussen vrouwen en nieuwe mannen op het migratiesucces van een man, en moeten de fitness consequenties van het gedrag van vrouwen tijdens migratie 
van mannen worden onderzocht. Tot nu toe zijn vrouwen vaak over het hoofd gezien bij migratie van mannen, maar in toekomstig onderzoek zouden apenvrouwen de aandacht moeten krijgen die ze verdienen. Want in tegenstelling tot wat vaak gedacht wordt, spelen vrouwen een actieve rol wanneer er een nieuwe man de groep in komt; zij hebben een duidelijke invloed op het succes van migrerende mannen. 


\section{Dankwoord}

Dit proefschrift was er niet geweest zonder de hulp en medewerking van een heleboel humane en niet-humane primaten. Ik begin met degenen die de belangrijkste bijdrage aan mijn proefschrift hebben geleverd: mijn geliefde aapjes! Ze hebben me vele uren bezig gehouden. Ik heb ervan genoten om uren naar ze te kijken. Ze hebben me vertederd. Ik heb om ze gelachen. Ze bleven me verbazen, als ze weer iets nieuws hadden bedacht of één of ander gek gedrag lieten zien. En stiekem heb ik ze ook wel eens vervloekt. Als ik een mooie strakke onderzoeksplanning had gemaakt, gooiden ze altijd roet in het eten. Maar het was zeker nooit saai. Ik prijs me gelukkig dat ik de afgelopen jaren met deze geweldige dieren heb mogen werken. Zonder hen was dit proefschrift er niet geweest.

Maar er zijn nog meer (minder harige) collega's, die een speciale vermelding in dit proefschrift verdienen. Jan en Liesbeth, bedankt dat ik onder jullie begeleiding mocht promoveren. Jullie hebben mij de vrijheid gegeven om van mijn promotieonderzoek helemaal mijn eigen project te maken. Ook stonden jullie altijd klaar om mij verder te helpen of van feedback te voorzien. Jullie vertrouwen in mijn kunnen en enthousiasme over mijn onderzoek hebben ervoor gezorgd dat ik het beste uit mijzelf heb kunnen halen.

Dan André en Saskia, met jullie bracht ik vele gezellige uren bij de aapjes door. Bedankt voor al jullie geduld en medewerking aan mijn onderzoek. Jullie hielden altijd rekening met mij en mijn onderzoek. André, ik heb ontzettend veel van je geleerd. Zonder jouw kennis en inzicht was dit proefschrift niet geworden wat het nu is. Een deel van je werkzaamheden (bijna een levenswerk) zijn in dit proefschrift vereeuwigd, daar kan je trots op zijn! Saskia, we hebben oneindig veel kopjes thee, repen chocola, winegums, pepernoten, zakken chips (af en toe moet dat kunnen) en flauwe grappen gedeeld. Wat zullen we mooi slank blijven nu we het BPRC allebei verlaten!

Annet, ook van jou en jouw eindeloze verhalen heb ik veel geleerd de afgelopen jaren. Je hebt me alles verteld over hoe het er bij het BPRC aan toe gaat en hoe het vroeger ging. Samen hebben we uren geploegd door oude data, waar twee hele mooie en belangrijke studies uit voort zijn gekomen. Dat was naast nuttig ook nog eens heel gezellig, net als onze gezamenlijke reisjes naar Londen, Praag en Portugal. Bedankt voor alles!

Dian en Lisette, mijn roomies in Rijswijk. Zonder jullie was het niet hetzelfde geweest. Het was geen moment saai op kantoor. We delen niet alleen onze liefde voor de aapies en welzijnsonderzoek, maar nog heel veel meer. Als ik het even niet meer zag zitten (of andersom), waren jullie daar altijd om mij weer op te beuren. En ik waardeer ook jullie eindeloze inzet (vooral die van Dian) bij het leegeten van de snoeppot. Ik ga jullie missen! 
Ook alle andere meiden op kantoor, met wie ik altijd zo lekker kon kletsen, verdienen een speciaal plekje. Het ging vaak over werk, maar ook heel vaak over hele andere dingen. Marit, Tina, Marlies, Deborah, Annemiek, Marieke en Daan, bedankt!

Francisca, zonder jouw hulp was mijn proefschrift niet zo mooi geworden! Bedankt voor de vele uren die je aan de vormgeving hebt besteed. Maar ook voor al je hulp bij het maken van mijn ingewikkelde figuren en de verschillende posters die je voor me hebt gemaakt.

Uiteraard zijn er nog veel meer collega's bij het BPRC die ik moet bedanken. Alle dierverzorgers en andere ASD-ers, die zo goed gezorgd hebben voor de aapjes en altijd rekening hielden met mij en de studenten tijdens de observaties, en tijdens de cursussen die ik heb begeleid. Ik ben bang dat ik mensen vergeet als ik namen ga noemen, maar ik ben jullie allemaal dankbaar! Jacqueline en Linda, dank voor jullie hulp bij de cortisol analyse. Ook veel dank aan mijn mede-promovendi, mijn mede-PV leden, mijn reisbuddy Babs, en alle anderen die ervoor hebben gezorgd dat ik mij thuis voelde bij het BPRC, mij hebben geholpen bij mijn onderzoek en altijd leuke en kritische vragen stelden na de vele praatjes die ik mocht geven.

Ook in Utrecht heb ik het voorrecht gehad om met leuke en fijne collega's samen te mogen werken. Marie José, Anne Marijke, Jorg, Henk en Karlijn, bedankt voor jullie vertrouwen en de gezellige woensdagen. Het was altijd fijn om op woensdag in Utrecht te zijn! Uiteraard verdient Han hier een speciale vermelding. Bedankt voor al je hulp bij de, soms ingewikkelde en duizelingwekkende data-analyse. Jij hebt me ontzettend veel geleerd! Ook veel dank aan alle studenten uit Utrecht die geholpen hebben met het verzamelen van de data: Marloes, Delphine, Anaïs, Sjoerd, Lisette, Yvette, Bas, Yualli, Kallirroi en Famke, bedankt voor jullie inzet!

Ten slotte, is daar ook nog het thuisfront. Ik ben jullie oneindig veel dank verschuldigd. Zonder jullie had ik mijn promotieonderzoek nooit kunnen doen.

Allerliefste Robert, zonder jouw steun en hulp had ik mijn promotieonderzoek nooit kunnen doen. Je hebt nooit geklaagd als ik weer eens laat thuis kwam, of in het weekend of de avonduren aan de slag moest. Na een dag hard werken, kan ik bij jou pas echt ontspannen. Jij weet precies wat ik nodig heb. Jouw trots motiveerde mij altijd om nog dat stapje extra te doen. Dank je wel voor alles, je betekent meer voor mij dan je kan bedenken. 
Lieve papa en mama, zonder jullie was ik nooit zo ver gekomen. Jullie hebben altijd in mij geloofd en achter mij gestaan. Bedankt voor alles wat jullie voor mij hebben gedaan. Dat geldt ook voor Karin en Daniël. Moet je eens zien waar dat gekke zusje dat altijd met die apen bezig was nu staat! Bedankt voor jullie steun en liefde. Die doctorstitel is ook een beetje voor jullie, om de familienaam op te sieren; Dr. Rox! Ik hou van jullie.

Ook veel dank aan alle andere vrienden en familie; Oma, Brenda, Ad, Anne-Marie, Jan, Tamara, Jerney, Willemijn, Marc, Max, en nog veel meer lieve mensen die mij altijd hebben gesteund en altijd naar mijn enthousiaste verhalen hebben willen luisteren. Zonder jullie, was dit proefschrift er niet geweest! 


\section{Curriculum Vitae}

Astrid Rox was born on the 9th of August 1990 in Apeldoorn, the Netherlands. As a child, she developed a great interest in animals and dreamed of making her career in animal science. After graduating from high school, she obtained a bachelor's degree in Biology at the Utrecht University. During one of the bachelor courses, Astrid observed the monkeys at the BPRC for the first time. This is when she decided to pursue a career in primatology. She enrolled in the Behavioural Ecology track from the Environmental Biology Masters programme at the Utrecht University, and focussed the majority of her master's research on the social behaviour and welfare of primates. Astrid started with an internship at Stichting AAP, where she studied remedies for self-injurious behaviour in traumatized macaques. Next, she wrote a literature thesis on butterfly migration and conservation. She finally obtained her master's degree after working at the Lilongwe Wildlife Centre in Malawi for nine months, where she conducted pre-release training and research on a troop of rehabilitated vervet monkeys that was being prepared for release back into the wild.

After she graduated, Astrid joined the BPRC to conduct her PhD research on the monkeys that made her pursue a career in primatology. During her PhD research she focused on the social behaviour and welfare of macaques, during the introductions of males into existing social groups. The results of that project are presented in this thesis. Simultaneously, Astrid worked as a teacher in behavioural biology at the Utrecht University.

\section{Publications}

Rox A, de Vries H, Louwerse AL, Sterck EHM (2018) Female social behaviour during three male introductions in captive groups of rhesus macaques. Appl Anim Behav Sci 207:89-97 . doi: 10.1016/j.applanim.2018.07.006

Rox A, van Vliet AH, Sterck EHM, et al (2019) Factors determining male introduction success and long-term stability in captive rhesus macaques. PLoS One 14:e0219972 




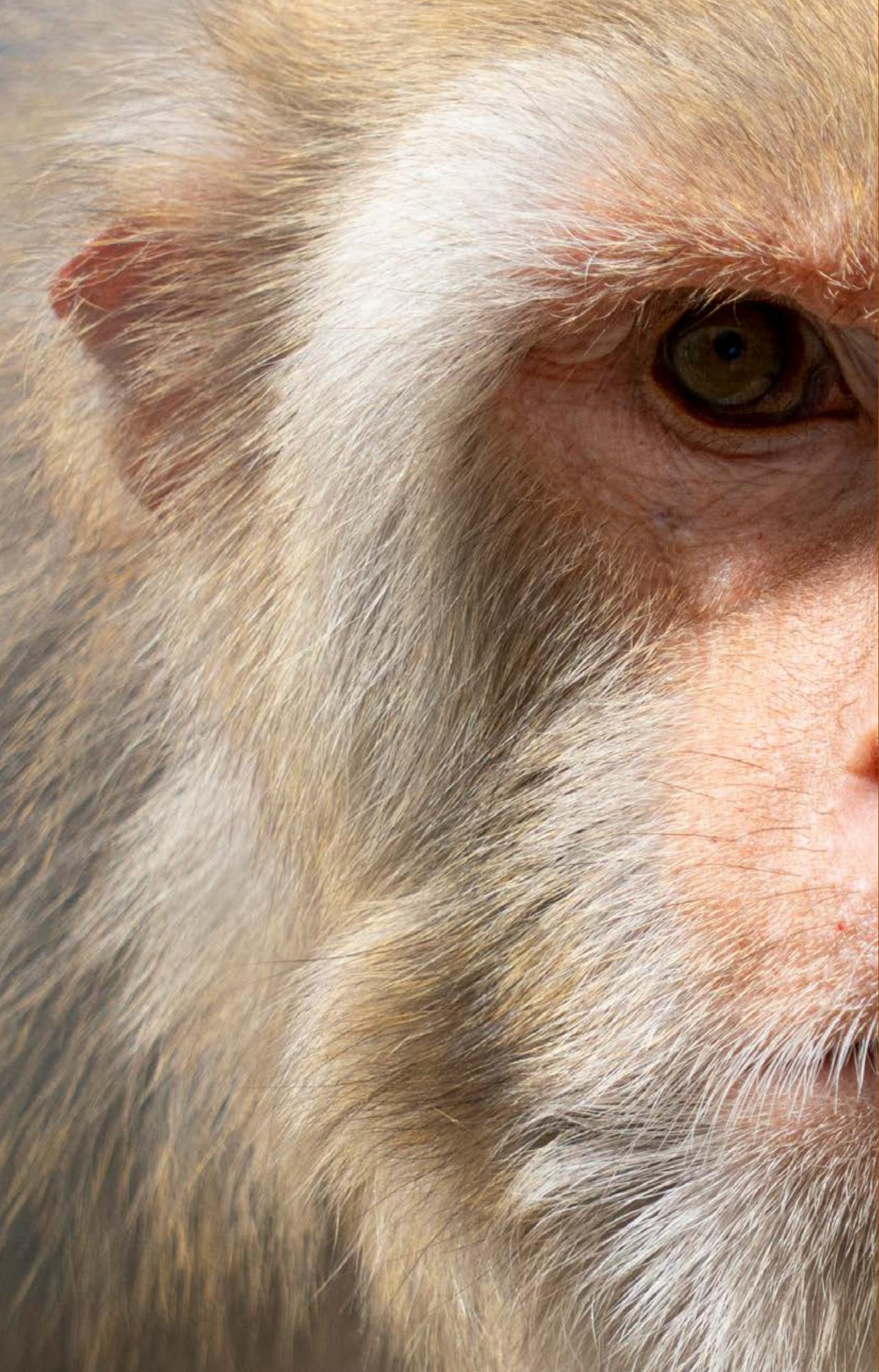

